a cura di

Claudia Lazzeri

\title{
Un carteggio \\ di fine secolo
}

Renato Fucini-Emilia Peruzzi

(1871-1899)

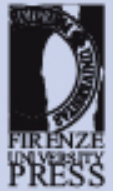




\section{Università degli Studi di Firenze}

Centro di Studi «Aldo Palazzeschi»

Letteratura e storia

2 


$$
\begin{aligned}
& \text { UNIVERSITÀ DEGLI STUDI DI FIRENZE } \\
& \text { CENTRO DI STUDI «ALDO PALAZZESCHI» }
\end{aligned}
$$

LETTERATURA E STORIA

1. Michele Monserrati, Le «cognizioni inutili». Saggio su "Lo Spettatore fiorentino" di Giacomo Leopardi, 2005 


\title{
Un carteggio di fine secolo: Renato Fucini - Emilia Peruzzi (1871-1899)
}

\author{
a cura di \\ Claudia Lazzeri
}

Firenze University Press 2006 
Un carteggio di fine secolo : Renato Fucini-Emilia Peruzzi (1871-1899) / a cura di Claudia Lazzeri. - Firenze : Firenze university press, 2006

(Letteratura e Storia, 2)

http://digital.casalini.it/8884534674

ISBN-10: 88-8453-467-4 (online)

ISBN-13: 978-88-8453-467-5 (online)

ISBN-10: 88-8453-468-2 (print)

ISBN-13: 978-88-8453-468-2 (print)

853.8 (ed.20)

Fucini, Renato - Peruzzi, Emilia - Lettere e carteggi

Direzione scientifica: Consiglio Direttivo del Centro di Studi «Aldo Palazzeschi»

Editing: Leonardo Raveggi

Impaginazione: Consorzio Editoriale

(C) 2006 Firenze University Press

Università degli Studi di Firenze

Firenze University Press

Borgo Albizi, 28

50122 Firenze, Italy

http://epress.unifi.it/

Printed in Italy 


\section{INDICE}

INTRODUZIONE

NOTA AL TESTO

Carteggio Renato Fucini - Emilia Peruzzi (1871-1899)

APPENDICE

INDICE DEI NOMI 



\section{INTRODUZIONE}

1. Non è possibile individuare con certezza la prima lettera che Renato Fucini e Emilia Peruzzi si scambiarono, come d'altronde non sono chiare le vicende che portarono il novelliere a frequentare il salotto Peruzzi ${ }^{1}$. Certamente questo dovette accadere fra la fine del 1870 e l'inizio del 1871 (il primo documento datato con sicurezza risale

1 Dei numerosi contributi su Renato Fucini, gran parte dei quali pubblicati su riviste e quotidiani, in particolare in occasione della morte e del centenario della nascita, ci limitiamo a segnalare i più recenti (oltre ad alcuni saggi inevitabili come B. Croce, Renato Fucini, in «La Critica», IV (1906), 1, pp. 249 254, poi in ID., La letteratura della nuova Italia, III, Bari, Laterza, 1915, pp. 141-148; C. SGROI, Renato Fucini, Firenze, Sansoni, 1943; E. Lama, Giudizio su Renato Fucini, Firenze, Bemporad-Marzocco, 1954; L. BALDACCI, Renato Fucini, in «Belfagor», XV (1960), 1, pp. 9-22, poi in ID., Letteratura e verità. Saggi e cronache sull'Ottocento e sul Novecento, Milano-Napoli, Ricciardi, 1963, pp. 72-88): L.G. SBROcCHI, Renato Fucini. L'uomo e l'opera, Firenze-Messina, D'Anna, 1977; F. BALDI, Un aspetto narrativo delle "Veglie di Neri" di Renato Fucini: la funzione dell'informatore, in «Italianistica», XVIII (1989), pp. 157-163; Un convegno a Monterotondo in onore di Renato Fucini, 12 aprile 1992, a cura di M. Giusti, Monterotondo Marittimo, Adver Agency, 1993; Omaggio a Renato Fucini. Atti del Convegno di studi (Pisa, 20 novembre 1993), a cura di G. Adami, Pisa, Goliardica, 1995; T. Iermano, Umorismo e leggerezza nei racconti di Renato Fucini, in «Esperienze Letterarie», XXIV (1999), 4, pp. 19-52; M. CICCUTO, Renato Fucini tra i pittori macchiaioli, in «Trasparenze», 10 (2000), 11, pp. 63-70; T. IERMANO, Invenzioni e aneddotica nella narrativa memorialistica di Renato Fucini, in "Critica Letteraria», XXX (2002), 2-3, pp. 573-591; ID., Esploratori delle nuove Italie. Identità regionali e spazio narrativo nella letteratura del secondo Ottocento, Napoli, Liguori, 2002, pp. 203-247 e pp. 249-278.

Molto è stato scritto anche su Emilia Peruzzi (Pisa 1826-Firenze 1900) e sul suo salotto. Anche in questo caso indichiamo i saggi più recenti. M.P. Cuccoli, Emilia Toscanelli Peruzzi, in «Rassegna Storica Toscana», XII (1966), 2, pp. 187-211; S. Fontana Semerano, P. Gennarelli Pirolo, Le carte di Emilia Peruzzi nella Biblioteca Nazionale di Firenze, in "Rassegna Storica Toscana», XXVI (1980), 2, pp. 187245, XXX (1984), 2, pp. 283-305; M. Dillon Wanke, De Amicis, il salotto Peruzzi e le lettere ad Emilia, in Edmondo De Amicis. Atti del convegno nazionale di studi (Imperia, 30 aprile-3 maggio 1981), a cura di F. Contorbia, Milano, Garzanti, 1985, pp. 55-145; M.I. Palazzolo, I salotti di cultura nell'Italia dell'Ottocento. Scene e modelli, Milano, Angeli, 1985; L.M. Fortunato De Lisle, The circle of Pear: Emilia Peruzzi and her salon - political and cultural reflections, issues and exchange of ideas in the new Italy. 1860-1880, Boston, U.M.I., 1988; U. RogaRI, Due regine dei salotti nella Firenze capitale. Emilia Peruzzi e Maria Rattazzi fra politica, cultura e mondanità, Firenze, Sandron, 1992; G. Rossi, Salotti letterari in Toscana. I tempi, l'ambiente, i personaggi, Firenze, Le Lettere, 1992; C. Ceccutr, Il salotto di Emilia Peruzzi, in Ubaldino Peruzzi, un protagonista di Firenze capitale. Atti del convegno di Firenze, 24-26 gennaio 1992, a cura di P. Bagnoli, Firenze, Festina Lente, 1994, pp. 17-33; M. T. MoRI, Salotti. La sociabilità delle élite nell'Italia dell'Ottocento, Roma, Carocci, 2000; E. BENUCCI, Introduzione a E. DE AMICIS, Un salotto fiorentino del secolo scorso, Pisa, ETS, 2002, pp. 27-55; S. SoldAnI, Emilia Toscanelli Peruzzi, o la passione della politica, in E. De Amicis, Un salotto fiorentino del secolo scorso, cit., pp. 11-26; R. MeLIs, "Una babelica natura": Sidney Sonnino, Emilia Peruzzi e il problema della lingua a Firenze dopo l'unità, in «Lingua nostra», LXIV (2003), 1-2, pp. 1-28. 
al 6 luglio 1871). All'epoca Fucini, giovane aiuto-ingegnere del comune di Firenze ${ }^{2}$, era impegnato nella composizione di sonetti in vernacolo pisano, destinati a ottenere un immediato successo anche al di fuori della cerchia delle sue amicizie, aprendogli, fra l'altro, le porte del salotto Peruzzi ${ }^{3}$. Non è chiaro chi introdusse l'autore maremmano in Borgo de' Greci. Una delle ipotesi più accreditate individua in Edmondo De Amicis il tramite tra Fucini e la Peruzzi ${ }^{4}$; altri hanno fatto il nome di Giovan Battista Giacomelli ${ }^{5}$, ricordato da De Amicis per il modo in cui recitava i sonetti fuciniani ${ }^{6}$, ma soprattutto nominato spesso dallo stesso Fucini in alcune sue lettere fino al 1876 con un sentimento di stima e di calda amicizia.

Fucini fece il suo ingresso nel salotto della signora Emilia quando si era appena concluso il suo periodo di maggior splendore, che aveva coinciso con gli anni di Firenze capitale, allorché veniva considerato una specie di "succursale del Parlamento» ${ }^{7}$ in quanto vi si riunivano i principali esponenti della Destra. Se per un salon l'intellettuale era un simbolo di prestigio, dando lustro col suo successo al salotto e ai padroni di casa ${ }^{8}$. A sua volta il salotto svolgeva per l'uomo di cultura non ancora famoso una funzione legittimante, attestandone le doti di cultura e il rilievo sociale? . Il letterato sapeva di trovarvi una dama protettiva, più spesso la padrona di casa, in grado di consigliare, e

2 Dalle lettere si intuisce come, con grande probabilità, Fucini sia stato un dipendente del Comune almeno fino al 1875, quando dovette perdere il lavoro, anche a causa della situazione finanziaria sempre più critica del Municipio. Tale ipotesi sembra essere confermata da Guido Biagi, che allude a un periodo successivo alla partenza della capitale da Firenze durante il quale Fucini, che aveva già pubblicato i Cento sonetti in vernacolo pisano presso l'editore Pellas (1872), avrebbe continuato a lavorare come aiuto-ingegnere (cfr. G. BIAGI, Da Neri Tanfucio a Renato Fucini, in ID., Passatisti, Firenze, "La Voce", 1923, pp. 31 e 38-39). Anche Alberto Niccolai afferma che lo scrittore avrebbe lavorato come dipendente del Comune dopo il 1872, sostenendo tuttavia nel medesimo saggio che il trasferimento della capitale a Roma comportò il suo licenziamento (si veda A. Niccolai, Renato Fucini. Saggio biografico critico, Pisa, Arti grafiche "Folchetto", 1921, pp. 31-32).

3 Ancora prima che i sonetti in vernacolo pisano venissero pubblicati in volume, Pietro Fanfani aveva dedicato a Fucini l'articolo Il poeta popolare, apparso sulla «Nuova Antologia», XVII (maggio 1871), 5, pp. 120-135.

4 Si veda a questo proposito E. BENUCCI, Introduzione a E. DE AMICIS, Un salotto fiorentino del secolo scorso, cit., p. 53. Tuttavia Lorenzo Gigli, nella sua biografia deamicisiana, non fa alcun riferimento al ruolo di tramite che lo scrittore ligure avrebbe svolto, insinuando, anzi, il dubbio di una sua iniziale invidia nei confronti di Fucini (si veda L. GigLI, Edmondo De Amicis, Torino, UTET, 1962, p. 247).

5 Si veda A. Niccolai, Renato Fucini. Saggio biografico critico, cit. p. 57; G. Varanini, Renato Fucini. Profilo critico, Pisa, Goliardica, 1955, p. 23. Riportiamo infine l'ipotesi secondo cui sarebbe stato Raffaelo Foresi l'artefice dell'incontro tra Fucini e la Peruzzi (cfr. D. Proietti, Fucini Renato in Dizionario biografico degli italiani, Roma, Istituto dell'Enciclopedia Italiana, 1998, vol. L, p. 675).

6 Si veda E. De Amicis, Un salotto fiorentino del secolo scorso, cit. p. 115: «Ricordo i primissimi [sonetti], scritti in foglietti volanti, che il Giacomelli leggeva alla signora Emilia e a pochi amici, dopo desinare, dando loro un colorito e una evidenza che neppure l'autore otteneva nel recitarli, e smorzando a un tratto la voce, come se gli mancasse il fiato, a tutte le frasi o parole non udibili da una signora; molte delle quali furono poi tolte nella stampa».

7 Cfr. E. De Amicis, Un salotto fiorentino del secolo scorso, cit., p. 72.

8 Lo dimostra, ad esempio, la lettera $\mathrm{V}$ del carteggio, in cui Fucini viene, più che invitato, pregato di presentarsi alla serata organizzata dal Circolo filologico in onore dell'imperatore del Brasile, in visita a Firenze.

9 Cfr. M. I. Palazzolo, I salotti di cultura nell'Italia dell'Ottocento. Scene e modelli, cit., p. 55. 
disposta, come nel nostro caso, a fungere quasi da press agent, ma anche un uditorio attento e sensibile, al cui giudizio sottoporre i propri lavor ${ }^{10}$ : personaggi del valore di Edmondo De Amicis, Sidney Sonnino, Francesco Genala e Vilfredo Pareto poterono godere del sostegno morale (e non solo) di Emilia ${ }^{11}$.

Il carteggio Fucini-Peruzzi permette innanzitutto di ripercorrere le tappe principali della carriera letteraria e della vicenda biografica dell'autore dei Cento sonetti. Le lettere sono ricche di indicazioni più o meno esplicite riguardo alla vita quotidiana di Fucini e della famiglia e consentono di ricostruire in maniera piuttosto precisa i suoi spostamenti, conosciuti finora in maniera abbastanza sommaria, nonché le sue vicissitudini lavorative comprese tra gli anni Settanta e gli anni Novanta dell'Ottocento, permettendo contemporaneamente di tracciare un ritratto dello scrittore scevro da quei tratti agiografici presenti in larga parte dei saggi critici che lo riguardano. Sono anni fondamentali nella vita dell'autore, che in questo periodo compose e diede alle stampe i suoi scritti più importanti: dai Cento sonetti in vernacolo pisano al primo esperimento in prosa di Napoli a occhio nudo; dai bozzetti delle Veglie di Neri a quelli di All'aria aperta. I primi anni dell'epistolario testimoniano dell'assidua frequentazione, da parte di Fucini, del salotto di Borgo de' Greci. Ci scorrono sotto gli occhi biglietti da visita di scarso interesse dal punto di vista del contenuto vero e proprio, ma che rivelano molto dei rapporti che dovettero intercorrere in questa fase fra i due protagonisti del carteggio. È un continuo susseguirsi di inviti a recarsi in visita presso i Peruzzi; la presenza di Fucini è costantemente richiesta nelle occasioni più importanti. A questi si aggiungono i biglietti per il teatro, le sollecitazioni perché il letterato assista a discorsi e conferenze, la segnalazione di articoli e libri da leggere, poiché Emilia si interessa affinché il suo protetto sia sempre al corrente di tutte le novità editoriali e delle più recenti notizie politiche ${ }^{12}$. Sarà questo un atteggiamento che la signora fiorentina terrà per tutto il corso dell'amicizia con Fucini e che, d'altronde, caratterizzerà tutti i suoi legami con i frequentatori del salon.

Fucini si sentì certo lusingato dalle tante attenzioni prestategli, cosa che spiega perché scriva alla sua amica bigliettini, in cui le rivolge appassionate espressioni di affetto amicale. ${ }^{13}$ Egli, tuttavia, non si limita a inviare dimostrazioni di gratitudine,

10 Cfr. M. T. Mori, Salotti. La sociabilità delle élite nell'Italia dell'Ottocento, cit., pp. 138-139.

11 Che la signora Emilia dispensasse consigli e incoraggiamenti ai giovani di talento è confermato anche da una lettera di De Amicis a Giuseppe Giacosa: «Caro Pin, perché non vai a Firenze? [...] Credi$\mathrm{mi}$, caro amico, se tu frequentassi quella signora per un mese, riacquisteresti piena fiducia in te stesso, ti sentiresti rinvigorito e rasserenato. Non c'è affetto di madre né di amico che in questi casi valga a rialzare un uomo dinanzi a se stesso quanto la parola brillante e gentile d'una signora coltissima, che indovina alla prima tutti i più riposti segreti d'un cuore d'artista, e gli rivela, per incoraggiarlo, delle qualità che egli non sapeva nemmeno di avere». Cfr. L. Gigli, Edmondo De Amicis, cit., p. 29.

12 Si veda A. Toscanelli Altoviti Avila, Emilia Toscanelli Peruzzi. Notizie e ricordi, cit., p. 19: «Mia zia amava in modo speciale di interessare gli amici, i piccoli, i grandi, a cerimonie, conferenze, discorsi, letture; e le piaceva tanto con le persone amiche il dividere le impressioni; e nei giovani, come ero io, perfezionare il gusto e lo spirito».

13 Cfr., ad esempio, le lettere LVI e XI. Non è raro che nella corrispondenza con Emilia venga usata l'immagine della donna-angelo. Si veda E. BENUCCI, Introduzione a E. DE AMICIS, Un salotto fiorentino del 
ma rende la sua benefattrice partecipe della propria carriera, tenendola al corrente delle proprie pubblicazioni e dei propri progetti ${ }^{14}$, discutendo con lei delle opere in corso di stampa, indicandola addirittura come diretta responsabile del suo volgersi alla prosa. In una lettera in nostro possesso, il ruolo svolto da Emilia nella "conversione" risulta determinante, poiché lei sola, col proprio intervento, con le proprie parole, avrebbe fatto nascere nell'animo del poeta quella insoddisfazione di sé che lo avrebbe indotto a impegnarsi nella prosa:

Cotesta sera, se ne rammenterà di certo, mi arrabattai tanto per mettere insieme la prefazione ai famigerati cento sonetti e dopo molte prove riuscite una peggio dell'altra ella mi disse e con tanta verità: - caro Fucini, quando scrive in versi ella è concettoso e vivace, ma nella prosa non la riconosco - . Ella diceva parole d'oro ed io ne tenni conto perché restai mortificato ${ }^{15}$.

Tuttavia, qualche anno dopo, nella rievocazione di Acqua passata la presenza di Emilia resta sullo sfondo, costituendo una sorta di mero pretesto allo svolgersi degli eventi, poiché ella risulta responsabile solo di avere indotto Fucini a scrivere la prefazione per i sonetti, non di avergli fatto aprire gli occhi dinanzi alle proprie deficienze:

L'ora d'andare a letto era finalmente arrivata; ma prima d'alzarsi, nacque un po' di discussione sui miei tentativi di prosa e fu amichevolmente osservato e deplorato che io, così fluido nello scrivere versi, stentassi tanto con la prosa e la scrivessi male ${ }^{16}$.

Cos'ha portato Fucini a ridimensionare in modo tanto vistoso il ruolo svolto da Emilia? Probabilmente accadde a lui ciò che prima era avvenuto ad altri: Fucini cercò infatti di sottrarsi all'ombra invadente e opprimente dei Peruzzi, avvertendo la necessità, nel suo libro di ricordi, dato alle stampe nel 1921, nonostante i debiti contratti nei confronti della nobildonna, di affermare la propria personalità di scrittore indipendentemente dall'influenza, di certo significativa e costante, che Emilia esercitò sulla sua carriera. Ricorre dunque in queste pagine di Acqua passata un'interpretazione tendente a sminuirne la tutela intellettuale, quasi una sorta di rimozione che tuttavia non si trasformerà mai in una dichiarazione aperta di distacco, come invece successe ad altri personaggi del "salotto rosso", giacché distacco netto non avrebbe potuto esservi, per l'ufficio di maestra che la Peruzzi svolse nei suoi confronti, decisamente meno determinante di quello svolto per altri illustri amici. Nelle lettere in nostro possesso, anche in quelle dei

secolo scorso, cit., p. 32: «Forse ella è una creatura straordinaria, così ispira sentimenti che escono fuori del cerchio dei sentimenti ordinari. Quello che la Vergine è tra l'umanità e Dio, ella lo è tra me e la virtù, tra me e la felicità, tra me e tutto quello a cui la mia anima aspira».

14 Si veda il caso del «barbaro epitalamio» (lettera XLIV), ossia i versi scritti in occasione del matrimonio del cognato Giorgio Roster con Emma Pellizzari, pubblicati sul «Corriere Italiano» nel gennaio del 1874 e di cui lo scrittore inviò alcune copie alla sua corrispondente

15 Cfr. la lettera LXXVII.

16 Cfr. R. Fucini, Acqua passata, in ID., Tutti gli scritti, Milano, Trevisini, 1946, pp. 511-512. 
primi anni, quando cioè Fucini era ancora nuovo al mestiere di scrittore, troviamo solo sparse indicazioni di consigli di carattere letterario dispensati da Emilia, e nessuno di natura linguistica. Si tratta di una notevole discordanza rispetto a quanto è dato leggere in altri carteggi della nobildonna finora dati alle stampe, che testimoniano invece una sua maggiore interferenza nella formazione dei giovani frequentatori del suo salotto. Vengono in mente alcuni dei nomi più illustri del suo entourage; sorvolando su De Amicis, il cui caso è già stato lungamente studiato ${ }^{17}$, potremmo fare il nome di uno dei protagonisti della vita politica italiana tra la fine dell'Ottocento e il primo quindicennio del Novecento, Sidney Sonnino, il cui apprendistato presso donna Emilia ebbe una durata pluriennale (dal 1872 al 1878), e vertè soprattutto su questioni linguistiche ${ }^{18}$. Niente del genere riscontriamo, invece, nella nostra corrispondenza. Certo, l'assidua frequentazione che, abbiamo detto, connotò con ogni probabilità i primi anni dell'amicizia, potrebbe spiegare l'assenza praticamente completa di ammaestramenti da parte di Emilia. Il fatto di vedersi tutti i giorni, o quasi, potrebbe aver indotto i due interlocutori a parlare faccia a faccia, e non per via epistolare, di questioni letterarie. Tuttavia, non abbiamo alcuna prova che dimostri questa ipotesi, che anzi sembra smentita da due fatti: il primo, che anche per gli anni successivi si lamenta la presenza di lettere in cui si parli di letteratura e, soprattutto, dell'opera fuciniana; il secondo, che con altri suoi giovani corrispondenti Emilia si intrattiene spesso nei bigliettini a discutere di questioni di stile, pur avendo la possibilità di affrontarle di persona. D'altra parte, la mancanza di indicazioni di tal genere nelle missive giunte fino a noi può essere spiegata anche dalla sostanziale discrepanza di gusti letterari fra i due corrispondenti, fatto nuovo rispetto a quanto testimoniano gli altri carteggi, soprattutto quello con De Amicis, che la Peruzzi aveva "plasmato, ispirato, gli aveva fornito esempi e suggerito argomenti» ${ }^{19}$. Il salotto di Borgo de' Greci sosteneva una politica culturale per così dire "conservatrice", fondata sul fiorentinismo alla Manzoni, su una letteratura che si facesse portavoce degli ideali della classe dirigente, venata di retorica, sentimentalismo e patetismo ${ }^{20}$. Fucini, invece, sembra essere interessato ad un modo diverso di scrivere, più "moderno" e attento alle

17 Circa l'importanza che Emilia ebbe sull'arte dello scrittore di Oneglia si possono consultare con una certa utilità L. Gigli, Edmondo De Amicis, cit., pp. 92-115; M. VAnnucci, De Amicis a Firenze. Le lettere dalla Spagna per la "Nazione» di Firenze. L'epistolario De Amicis-Peruzzi, Firenze, Istituto Professionale "Leonardo da Vinci", 1972-1973; M. Dillon Wanke, De Amicis, il salotto Peruzzi e le lettere ad Emilia, cit., pp. 55-145; S. Spandre, Le lettere di Edmondo De Amicis ad Emilia Peruzzi: l'evoluzione di un rapporto e di una personalità, in «Studi piemontesi», XIX (1990), 1, pp. 31-49; L. TAMBURINI, Confidenze tra signore: lettere inedite di Teresa Busseti a Emilia Peruzzi, in "Studi piemontesi», XXI (1992), 23, pp. 485-510; E. BENUCCI, Introduzione a E. DE AMICIS, Un salotto fiorentino del secolo scorso, cit. pp. 30-37.

18 Sull'amicizia tra Sonnino e la Peruzzi si veda P. CARluCCI, Un'amicizia controversa: Sidney Sonnino ed Emilia Peruzzi (1872-1878), in Ubaldino Peruzzi, un protagonista di Firenze capitale, a cura di P. Bagnoli, cit., pp. 161-177; Lettere di Sidney Sonnino ad Emilia Peruzzi, 1872-1878, a cura di P. Carlucci, Pisa, Scuola Normale superiore, 1998; R. Melis, "Una babelica natura”: Sidney Sonnino, Emilia Peruzzi e il problema della lingua a Firenze dopo l'unità, cit.

19 Cfr. L. Gigli, Edmondo De Amicis, cit., p. 105.

20 Interessanti considerazioni sul programma linguistico e, più in generale, culturale propugnato dal salotto Peruzzi sono contenute in R. Melis, "Una babelica natura": Sidney Sonnino, Emilia Peruzzi e il problema della lingua a Firenze dopo l'unità, cit. 
riflessioni che, proprio negli anni Settanta, vedono impegnati alcuni fra i più importanti scrittori italiani. Se consideriamo le opere della maturità, da Napoli a occhio nudo ai bozzetti delle Veglie di Neri e di All'aria aperta, vediamo come esse siano legate al dibattito, sviluppatosi in particolar modo tra 1877 e il 1880, sul naturalismo francese e sul roman expérimental di Zola. La corrispondenza intercorsa tra Fucini e due grandi nomi della stagione verista, Capuana e Verga ${ }^{21}$, nonché i rapporti intrattenuti con i collaboratori della «Rassegna Settimanale» bastano a testimoniare della sua attenzione per la nuova letteratura che si andava affermando in quegli anni. Certo il regionalismo fuciniano manca di una consapevole riflessione sui modi di rappresentazione del vero così come nel miglior verismo degli scrittori siciliani, e poi la limitata prospettiva di indagine ridotta ad alcuni aspetti soltanto del mondo provinciale, come pure l'uso di certe, elementari tecniche narrative, ad esempio l'ampio e frequente ricorso alla figura del narratore-testimone, rivelano un sostanziale misconoscimento dell'opera e delle teorie di un caposcuola come Emile Zola. Tuttavia, non possiamo non notare lo stridente contrasto tra i gusti della signora Emilia e gli ironici giudizi del nostro nei confronti delle opere sottopostegli dall'amica. Non solo Fucini bolla come «buco nell'acqua» il progetto del Novo vocabolario della lingua italiana secondo l'uso di Firenze a cui stavano lavorando alcuni dei più assidui frequentatori del salotto ${ }^{22}$, ma, diversi anni dopo, esprime un'opinione completamente negativa su Arabella di Emilio De Marchi, un romanzo che era invece molto apprezzato dalla protagonista del nostro carteggio ${ }^{23}$. Ora, che il libro di un epigono della tradizione manzoniana e portavoce di una morale sostanzialmente antipositivistica, destasse un moto di insofferenza nel nostro scrittore non è cosa di cui meravigliarsi, se solo si legga ciò che, circa vent'anni prima, Fucini aveva asserito riguardo ai naturalisti e agli scienziati in un significativo biglietto:

Non posso negare che questi naturalisti hanno, da un pezzo in qua, pochi discorsi e buoni, e degli argomenti parecchio stringenti senza tante spampanate rettoriche. Io gli voglio bene ai naturalisti e ai cultori delle scienze fisiche in generale. Sono quelli che quando muoiono lasciano poco rumore di campane ma pei quali resta il vapore, il telegrafo a dichiararli i primi se non gli unici benemeriti del vero progresso ${ }^{24}$.

Il nostro poeta, dunque, venne accolto benevolmente nell'aristocratico salotto, dove portò "un soffio vivo d'aria primaverile» ${ }^{25}$, e dove ricevette i primi incoraggiamenti a proseguire per la strada intrapresa ${ }^{26}$. Fin qui, perciò, un rapporto

21 Cfr. A. Navarria, Lettere del Capuana e del Verga a Renato Fucini, in «Belfagor», XV (1960), 1, pp. 466-468.

22 Cfr. la lettera XLIX.

23 Si veda la lettera CL.

24 Si veda la lettera XXXVI.

25 Cfr. E. DE Amicis, Un salotto fiorentino del secolo scorso, cit., p. 117.

26 De Amicis narra come, per mezzo di Giovan Battista Giorgini, Fucini avesse ottenuto l'approvazione di Alessandro Manzoni, e, soprattutto, come lo stesso Giorgini gli avesse consigliato "[...] "di fuggire i letterati come la peste" di mandare al diavolo i pedanti che già cominciavano a esortarlo a 
improntato alla gentilezza e alla cortesia, con una punta di deferenza nei riguardi di donna Emilia dovuta alla consapevolezza dell'onore concessogli nel frequentare l'eletto circolo.

La situazione subisce un cambiamento nel momento in cui Fucini perde il lavoro di aiuto-ingegnere. Ha inizio per lui un anno penoso, il 1876, durante il quale diventa giocoforza trovare al più presto un nuovo impiego che gli permetta di mantenere la famiglia (Fucini, che si è sposato a ventiquattro anni, ha già due figlie, Ida e Rita). Naturale chiedere aiuto agli amici più influenti che ha in città, i Peruzzi. Ma succede qualcosa: le sue affannose preghiere sembrano cadere nel vuoto ${ }^{27}$, mentre sappiamo che Emilia era sempre stata sollecita nel correre in soccorso dei suoi protetti in difficoltà ${ }^{28}$. Nonostante l'intervento richiesto ancora una volta al marito, non si sa con quanta perentoria urgenza, i contatti procurati da Ubaldino vanno tutti a vuoto, mentre l'unico incarico che riceve anche grazie all'intervento dei Peruzzi è quello di recarsi a Napoli per studiare le condizioni in cui versa la città ${ }^{29}$ (in questo caso, però, è solo l'intervento di Emilia a essere determinante, mentre Ubaldino non sembra partecipare in alcun modo), inducendo Pasquale Villari, dopo il rifiuto di De Amicis, a inviare nella città partenopea il comune amico in difficoltà ${ }^{30}$. Un viaggio, questo, che si dimostrerà fondamentale per Fucini, perché da tale esperienza nascerà Napoli a occhio nudo, il suo primo lavoro in prosa (se si eccettua il bozzetto Il Matto delle Giuncaie, apparso nel 1876 sulla «Nuova Antologia»), dato alle stampe nel 1878 e oggi considerato forse l'opera più riuscita dell'intero corpus fuciniano, sicuramente una delle più lucide denunce sociali della nostra letteratura tardo ottocentesca.

Tornato dalla Campania, si ripresenta il problema, ormai non più procrastinabile, di trovare un impiego stabile e ben remunerato. I Peruzzi non si spendono più di tanto in suo favore e lo scrittore, in una lettera datata 5 ottobre 1877 e indirizzata all'amico Giovanni Procacci, che gli aveva procurato il lavoro di insegnante di belle lettere a Pistoia, manifesta il proprio risentimento:

lasciar la poesia vernacola, come un'arte inferiore, e a darsi alla satira italiana, di proseguire per la via su cui s'era messo fin che avesse voglia e forza e piacere d'andare avanti [...]». Cfr. E. DE AMICIs, Un salotto fiorentino del secolo scorso, cit., p. 117.

27 Cfr. la lettera LXXviII.

${ }^{28}$ Si veda a questo proposito quanto scritto dalla nipote Angiolina Toscanelli Altoviti Avila: «Io non ho memoria che una domanda di aiuto, per quanto strampalata, o la raccomandazione più ardua, se giusta, fossero ricevute da lei con un atto d'impazienza. Tutte le accoglieva; e ricordo le dolci sue persecuzioni allo zio Ubaldino per qualche onorificenza, per qualche caritatevole impiego [...]» (cfr. A. TosCANelli Altoviti Avila, Emilia Toscanelli Peruzzi. Notizie e ricordi, cit., pp. 25-26).

29 Dal carteggio risulta anche che la signora Emilia si adoperò per trovare a Fucini un lavoro come insegnante o come correttore presso la "Gazzetta d'Italia» (cfr. la lettera LXXVII: «Sappia che appunto avevo scritto per Lei sia come Insegnante, sia come correttore alla Gazzetta d'Italia [...]»).

30 Si veda, a questo proposito, E. BENUCCI, Introduzione a E. DE AMICIS, Un salotto fiorentino del secolo scorso, cit. p. 53. La Peruzzi fornì allo scrittore anche una serie di lettere di presentazione per le più illustri famiglie napoletane; si veda L. G. Sвrocchi, Fucini-Fortunato: Napoli a occhio nudo, in "Critica Storica», XII (marzo 1975), 1, p. 168. 
[I Peruzzi] mi hanno sempre dimostrato la più simpatica amicizia a pranzo e a parole, ma in fatti sono stati sempre duri. Il Peruzzi, come Sindaco, come Deputato, come exMinistro, e come Ubaldino avrebbe potuto farmi, dall'oggi al domani, una posizione in Firenze e non me l'ha fatta... Io ho durato un pezzo a raccomandarmi a lui ed alla signora Emilia, ed ora mi basta. Il tornare ora alla carica con loro sarebbe per me una specie di umiliazione, alla quale il mio debolissimo orgoglio non mi permette abbassarmi ${ }^{31}$.

La delusione per lo scarso ruolo avuto dai due coniugi nella vicenda traspare anche dalle lettere ad Emilia, che si fanno meno frequenti. In una di esse tuttavia lo scrittore, annunciandole di aver ottenuto un posto di insegnante a Pistoia, sembra alludere ironicamente alle mancate premure dell'amica dalla quale si sarebbe atteso ben altro interessamento:

P.S.: Una imperdonabile dimenticanza mi accorgo d'aver commesso con la furia e le faccio le mie più grandi scuse. Ho dimenticato di ringraziarla delle premure che ultimamente si è data per trovarmi una occupazione in Firenze. La ringrazio di grandissimo cuore perché con quel pensiero ha toccato la corda più sensibile dell'animo mio, ma ormai è finita ${ }^{32}$.

Difficile capire comprendere le ragioni di questo comportamento da parte dei Peruzzi, sempre pronti a intervenire a favore dei loro amici, e che, in altre circostanze, si dimostreranno solleciti nell'accogliere le richieste di aiuto e le raccomandazioni presentate loro da Fucini. Possiamo solo ipotizzare che le antipatie che Ubaldino si era attirato proprio nel marzo del 1876 a causa della caduta della Destra e, inoltre, le condizioni finanziarie sempre più difficili del Comune di Firenze lo avessero indotto a essere in generale poco disponibile a richieste e raccomandazioni e dunque anche alle pressanti preghiere dello scrittore di Monterotondo ${ }^{33}$. Probabilmente Fucini non capì, o non volle capire, la drammatica circostanza in cui si trovava il sindaco di Firenze, il quale per la crisi del Comune, nell'arco di alcuni anni, perse la maggior parte dei suoi beni, compresa la dote di Emilia, costretto peraltro ad abbandonare per sempre Borgo de' Greci e a ritirarsi nella villa dell'Antella ${ }^{34}$.

Dunque, suo malgrado, Fucini dovette lasciare la brillante società fiorentina per chiudersi nella piccola e provinciale Pistoia. Si trattò di un vero e proprio sacrificio, che rischiò di minare la sua autostima e il suo orgoglio di scrittore ${ }^{35}$, come si

31 Si veda A. Chitr, Ricordi pistoiesi di Renato Fucini, in «Bullettino storico pistoiese», XLV (1943), 1-2, p. 8.

32 Cfr. lettera LXxxviII.

33 Sulla caduta della Destra e sulla crisi finanziaria del Comune di Firenze si veda Z. CiUfFolettri, Ubaldino Peruzzi, la caduta della Destra e la «questione di Firenze», in Ubaldino Peruzzi un protagonista di Firenze capitale, a cura di P. Bagnoli, cit., pp. 267-291.

34 A partire dal 1876, la situazione economica dei Peruzzi si fece di anno in anno più difficile (cfr. T. Giacalone Monaco, Introduzione a V. Pareto, Lettere ai Peruzzi, cit., p. XXXVI).

35 Si veda, ad esempio, la lettera XCIX: «M'avevan fatto credere che avessi un po' di criterio e qualche attitudine, ma non ci credo più». 
legge nella lettera XCIV, dove la partenza da Firenze è definita una «ferita toccata nell'amor proprio». L'accoglienza ricevuta a Pistoia fu però calorosa ${ }^{36}$ e lo scrittore divenne intimo di alcune delle famiglie più in vista, come i Bartolini, i Niccolai, i Rossi-Cassigoli, in casa dei quali si discuteva di cose letterarie e d'arte ${ }^{37}$. Purtroppo, le nostre lettere non aiutano a chiarire quale fosse la vita di Fucini e della sua famiglia a Pistoia, essendo quasi sempre prive di riferimenti a questo riguardo ${ }^{38}$. Nonostante fosse considerato una personalità di spicco e che, per questo, venisse accolto nei ritrovi più illustri della città, a Fucini non dovette sfuggire lo stridente contrasto tra la sonnacchiosa società pistoiese e quella fiorentina, certo più vivace e stimolante. Pur presentandosi l'occasione di vivere una vita a contatto con la natura, immerso in quelle attività come la caccia e la pesca che prediligeva, egli non dimentica mai nelle sue lettere di lamentarsi della noiosa vita di provincia che lo inibisce, rendendolo svogliato, tanto da tralasciare la lettura dei giornali ${ }^{39}$. Anche il lavoro non lo soddisfa, ne sente tutta l'inutilità e comprende la propria inadeguatezza al compito affidatogli, soprattutto per gli ostacoli incontrati, legati per lo più alle polemiche sorte intorno alla necessità di abolire o meno le scuole tecniche ${ }^{40}$. Unico conforto, gli studi: nei primi tempi del soggiorno pistoiese, Fucini lavora incessantemente a Napoli a occhio nudo e, contemporaneamente, inizia la collaborazione alla "Rassegna Settimanale», sulla quale, tra il 1878 e il 1882, pubblicherà quasi tutti i bozzetti raccolti poi sotto il titolo Le veglie di Nerit ${ }^{41}$, continuando a informare la Peruzzi dei lavori pubblicati, in rivista o in volume ${ }^{42}$. Questo quadro sembra sfatare l'immagine, diffusa da una certa critica di inizio Novecento, di un Fucini modesto e soddisfatto da una vita sana e tranquilla ${ }^{43}$. Che a lui non interessasse di apparire sulla scena dell'arte non sembra

36 Si veda la lettera LXXXIX: «A Pistoia sono stato accolto con molta festa e questo ha molto contribuito a farmi guardare con più calma l'idea d'andarmi a seppellire in provincia e a mettermi un po' in rialzo il morale, ma ho avuto dei gran giorni neri».

37 A proposito delle relazioni strette da Fucini a Pistoia cfr. A. CHITI, Ricordi pistoiesi di Renato Fucini, cit., in particolare le pp. 11-15.

38 Unica lettera in cui Fucini fa riferimento a un'amicizia stretta a Pistoia è la XCI, dove si legge dell'incontro con l'abate Giuseppe Tigri, un tempo insegnante di Ubaldino.

39 Cfr. la lettera XCIX.

40 Alcuni giornali pistoiesi di orientamento conservatore e reazionario avevano infatti lanciato una vera e propria campagna per la chiusura delle scuole tecniche dove insegnava Fucini cfr. D. MARRACCINI, Renato Fucini a Pistoia, in «Bullettino Storico Pistoiese», LXXXIV (1982), p. 104.

41 Le uniche eccezioni sono costituite da Scampagnata, che venne inviato dall'autore direttamente in tipografia, da Dolci ricordi, comparso sulla "Domenica del Fracassa» nel 1885 e Passaggio memorabile, inseriti nell'edizione Hoepli del 1890, oltre che da Nonno Damiano e da La maestrina, entrati a far parte della raccolta solo nell'edizione "vociana” del 1921, uscita postuma.

42 Si vedano le lettere XCIV, C, CII e CIV, nelle quali Fucini fa riferimento rispettivamente alla stampa di Napoli a occhio nudo, del sonetto Le 'ampane e dei bozzetti Nonno in Maremma e La fatta.

43 Si vedano a questo proposito R. Simoni, I settant'anni di Renato Fucini, in "Corriere della Sera", 8 aprile 1913; ID., La morte di Renato Fucini, ibid., 26 febbraio 1921; P. BARGELLINI, A veglia con Fucini, Firenze, Vallecchi, 1943, p. 89; G. TiтTA Rosa, Renato Fucini, in ID., Secondo Ottocento. Da Nievo a D’Annunzio, Milano, Garzanti, 1947, p. 149. 
sostenibile dopo aver letto le lettere in cui si lamenta della tiepida accoglienza fatta a Firenze al suo Napoli a occhio nudo o dove si rammarica di essersi illuso di avere le doti giuste per poter emergere nel mondo letterario ${ }^{44}$.

La situazione sembra migliorare solo quando, nel 1879, ottiene l'incarico di ispettore scolastico, come sostituto di Mario Manfroni ${ }^{45}$. Si susseguono lettere in cui il nostro esprime tutta la sua felicità per il nuovo impiego, che sente veramente confacente a sé e che gli permette una maggiore libertà. Certo, il lavoro non gli lascia che poco tempo libero, e tuttavia egli sembra perfettamente soddisfatto. È questo un dato sottolineato da larga parte della critica, che indica gli anni d'ispettorato tra $\mathrm{i}$ più felici mai vissuti dallo scrittore, soprattutto per il piacere di stare a contatto con quella natura che aveva imparato ad amare da bambino, traendovi ispirazione per i propri bozzetti. Questo lungo periodo (Fucini sarà ispettore scolastico dal 1879 al 1900) fu fondamentale per la sua maturazione umana e culturale, offrendogli il destro di osservare attentamente il mondo poi ritratto nelle prose delle Veglie di Neri e di All'aria aperta ${ }^{46}$.

Per quanto riguarda lo stato della relazione tra lo scrittore e i Peruzzi, è interessante sottolineare un' inversione di tendenza rispetto al 1877, dal momento che all'indifferenza si sostituisce una certa attenzione manifestata allorché il giovane sostituto ispettore scolastico chiede il loro intervento per ottenere il posto lasciato vacante dal Manfroni. Le missive scambiate in questa circostanza sembrerebbero confermare la nostra ipotesi secondo la quale l'aiuto dei Peruzzi sarebbe venuto meno in un primo tempo in ragione delle difficoltà che Ubaldino aveva incontrato sia come deputato che come sindaco di Firenze. Adesso, e fin dal giugno del 1878, il Peruzzi non è più sindaco (essendosi dimesso insieme all'intero consiglio comunale nella speranza, com'è noto, che tale gesto favorisse la risoluzione della questione fiorentina) ${ }^{47}$, permettendo così un interessamento più efficace e una sollecitudine nuovi nei confronti dell'amico, per cui nel giro di pochi giorni, ossia tra il 29 gennaio del 1880 e il 6 febbraio dello stesso anno ${ }^{48}$, la sua richiesta all'ex-sindaco viene soddisfatta. Fucini mostra quasi di spaventarsi per l'eccessiva foga con cui Ubaldino ed Emilia si erano occupati di una faccenda per lui

44 Cfr. la lettera XCIV.

45 Mario Manfroni frequentò i corsi universitari a Innsbruck, Monaco e Vienna. Dopo aver sostenuto gli esami di libera professione a Venezia, tornò Trento per dirigere la rivista «Il Trentino». Nel 1874 entrò a servizio del governo italiano, prima come Ispettore scolastico a Pistoia, poi come Commissario Regio al Collegio Principe di Napoli a Assisi e, infine, come Direttore delle Regie Scuole Magistrali superiori di Catanzaro, Belluno e Lodi. Dal 1887 divenne Capo sezione al Regio Ispettorato delle strade ferrate.

46 Sfortunatamente, le lettere gettano ben poca luce su questo aspetto, dal momento che Fucini allude solo raramente agli avvenimenti cui ha assistito durante le sue ispezioni scolastiche. Ci sono, tuttavia, due importanti eccezioni, costituite dalle lettere CXXV e da quelle riguardanti la statua a Vincenzo Chiarugi (CLII, CLX, CLXI), che potrebbero aver ispirato due bozzetti, La maestrina, inserito postumo nelle Veglie di Neri, e Il monumento, contenuto in All'aria aperta. 283.

47 Cfr. Z. Ciuffoletti, Ubaldino Peruzzi, la caduta della Destra e la "questione di Firenze», cit., p.

48 Si vedano le lettere CII, CIII, CIV. 
così delicata ${ }^{49}$. Il rapporto con i Peruzzi, e con la signora Emilia in particolare, ritorna alla cordialità che lo aveva connotato nei primi anni ${ }^{50}$, e i due amici, confidando nelle conoscenze che Ubaldino può mettere in campo, cominciano a progettare il rientro dello scrittore a Firenze. Di fronte all'entusiasmo di donna Emilia, Fucini mantiene un atteggiamento più distaccato ${ }^{51}$, probabilmente perché, in attesa dell'occasione giusta per riportarsi nel capoluogo toscano, voleva ottenere un posto adeguato, che gli consentisse di vivere agiatamente e che lo soddisfacesse pienamente ${ }^{52}$. Infatti, non appena si presentò l'occasione, ricorse nuovamente ai Peruzzi, chiedendo una raccomandazione presso il provveditore Bolasco per essere trasferito al circondario di San Miniato, cosa che gli avrebbe permesso di amministrare con maggior agio le sue proprietà ${ }^{53}$. Anche in questo caso l'intervento dei Peruzzi sembra dare i suoi frutti, se è vero, come sostiene la Marraccini ${ }^{54}$, che tra il 1883 e il 1884 egli è effettivamente spostato al circondario di San Miniato, benché, contrariamente a quanto la stessa sostiene nel suo articolo, Fucini continui a risiedere a Pistoia, come dimostrano alcune lettere relative proprio a questi anni e spedite da quella città ${ }^{55}$.

D'altra parte, in Emilia non era mai venuto meno l'interesse per le vicende personali dell'amico e la cordialità nei suoi confronti, nemmeno quando, in seguito al licenziamento da aiuto-ingegnere, Fucini, in cerca di lavoro, aveva mostrato una certa freddezza nei confronti dei Peruzzi. Benché lontano da Firenze, ella si mostra sempre desiderosa e, diremmo, quasi ansiosa di rivederlo, di parlare con lui dei gravi problemi che affliggevano Firenze e l'Italia proprio come era accaduto nei primi anni Settanta, cioè nel periodo in cui lo scrittore era stato più assiduo nel suo salotto. Lo sollecita a interessarsi ad argomenti di più stretta attualità, con una particolare attenzione verso la situazione fiorentina che le sta, com'è ovvio, a cuore in modo particolare, consigliandogli la lettura di libri e articoli di giornale ${ }^{56}$, ma, soprattutto, proponendogli di meditare sulle gravi ingiustizie patite da Ubaldino, ritenuto l'unico vero colpevole della disastrosa crisi finanziaria del Comune di Fi-

49 Cfr. lettera CIV: «Badiamo, per carità, che non accadano malintesi che potrebbero essermi dannosissimi. Lo ripeto - Succedere al Manfroni quando il Manfroni per avanzare nella sua carriera avrà lasciato scoperto l'Ispettorato di Pistoia -. [...] Se al ministero avessero inteso diversamente le mie premure, ne sarei desolato».

50 Si veda ad esempio la lettera CVI: «Povero Sig. Ubaldino quanto ha fatto per me! Ma quando m'accosterò a lui si guardi bene perché non garantisco di poter resistere alla tentazione di dargli un abbraccio come lo darei a mio padre».

51 Cfr. la lettera CVI.

52 Molti degli antichi compagni del salotto di Borgo de' Greci avevano infatti proposto a Fucini incarichi di insegnante in varie scuole fiorentine, ottenendo solo dei rifiuti, e possiamo anzi immaginare che la signora Emilia non fosse del tutto estranea a questi interessamenti (cfr. A. CHITI, Ricordi pistoiesi di Renato Fucini, cit., p. 18).

53 Si veda la lettera CVIII.

54 Cfr. D. Marraccini, Renato Fucini a Pistoia, cit., p. 106.

55 Cfr. le lettere CXVI, CXVII, CXVIII.

56 Si vedano le lettere LXXIV e XCVI. 
renze. Da parte sua lo scrittore, pur manifestando la propria solidarietà a Ubaldino anche per le aggressioni fisiche e verbali ricevute ${ }^{57}$, non volle esporsi più di tanto in suo favore.

A differenza di quanto sostenuto dalla Marraccini nel suo articolo, ossia che lo scrittore, dopo una breve pausa di circa due anni, si sarebbe riportato a Pistoia nel 1885 , per restarvi fino al $1888^{58}$, le lettere alla Peruzzi dimostrano invece come il nostro si trovasse a Firenze fin dal maggio del $1886^{59}$. Purtroppo, le vicende biografiche di Fucini sono singolarmente poco note, e in più di un caso abbiamo riscontrato errori talvolta grossolani circa le date di pubblicazione delle sue opere, oltre a lacunose indicazioni su momenti anche fondamentali della vita ${ }^{60}$, anche se le lettere del carteggio da noi preso in esame non sempre consentono di venire a capo di quelle aporie biografiche che pure adombrano. Tuttavia, è un fatto che il nostro visse dal 1886 fino al 1888 a Firenze, e che solo nel 1889 abbandonò la città per trasferirsi con la famiglia ad Empoli, come testimonia la lettera CXXXV, datata 1 dicembre 1889 e spedita appunto da questa città.

Il ritorno a Firenze è accolto con grandissima gioia da Emilia, che subito invita Fucini a farle visita all'Antella, sperando che l'amico ricominciasse a frequentare assiduamente il suo salotto. La realtà si mostra ben presto assai diversa ${ }^{61}$. Lo scrittore, già a un mese dal suo trasferimento a Firenze, è costretto a scusarsi per l'impossibilità di farle visita ${ }^{62}$. Nel leggere le sue ripetute giustificazioni, viene il dubbio che Fucini non avesse più alcun interesse per il salotto, che nel corso degli anni era andato perdendo smalto, continuando a recarvisi solo per dovere di gratitudine. I circa due anni che Fucini trascorse ancora a Firenze, perciò, non sortirono quel riavvicinamento auspicato in specie da Emilia.

Ovvio, dunque, che la lontananza dello scrittore da casa Peruzzi si sia fatta ancor più grave negli anni successivi al 1889, quando egli, sempre per motivi di lavoro, è costretto a trasferirsi a Empoli. Continuano le promesse di Fucini a recarsi quanto

57 Cfr. le lettere XCVIII e XCIX.

58 Si veda D. Marraccini, Renato Fucini a Pistoia, cit., pp. 106-107.

59 Cfr. la lettera CXII.

60 Solo per fare qualche esempio: il Biagi sostiene che Fucini sarebbe rientrato a Firenze nel 1882, notizia che viene riportata anche da Luigi Pescetti: cfr. G. BIAGI, Renato Fucini nella vita e nell'arte, in «La lettura», XXII, 1922, pp. 321-331, poi in ID, Passatisti, Firenze, "La Voce”, 1923, p. 80; L. PesCeTTI, Renato Fucini uomo e poeta, in "Nuova Antologia», CDXXVII (1 maggio 1943), p. 16; il Niccolai posticipa l'uscita dell'edizione Barbèra dei Cento sonetti in vernacolo pisano al 1886 (cfr. A. NiccolaI, Renato Fucini. Saggio biografico critico, cit., p. 31); Alfredo Chiti allude a un intervallo nel soggiorno di Fucini a Pistoia situato tra il periodo in cui è insegnante e quello in cui diventa ispettore scolastico, ma di ciò non si ha traccia nelle lettere (si veda A. CHITI, Ricordi pistoiesi di Renato Fucini, cit., p. 19).

61 Cfr. la lettera CXxxIv: «Ho saputo la sua intenzione di venire all'Antella, ma in conclusione non ci vediamo da gran tempo, e mi duole che sia un ritorno a Firenze di cui si gode pochissimo».

Si veda inoltre la lettera CXXVI: "Ci accorgiamo troppo poco, caro Fucini, che Ella sia tornato a Firenze».

62 Si veda la lettera CXXIV, datata 27 giugno 1886 (Fucini era rientrato in Firenze agli inizi di maggio dello stesso anno): «Il mio biglietto tiene luogo di una visita di congedo che con mio grande rincrescimento non ho potuto fare in persona». 
prima in visita agli amici, ma si tratta per lo più di impegni che solo raramente vengono mantenuti, mentre anche la corrispondenza con Emilia si fa più rarefatta. Per più di un anno tra i due non viene scambiata nemmeno una missiva, e dal 29 dicembre 1889 si salta fino al 3 gennaio 1891, quando lo scrittore scrive finalmente alla sua amica, chiedendole perdono per il lungo silenzio ${ }^{63}$.

Nel frattempo le condizioni di salute di Ubaldino peggiorano, tanto che le visite degli amici vengono interrotte ${ }^{64}$, e la sua scomparsa, avvenuta il9 settembre 1891 , lascia Emilia distrutta $^{65}$, mentre il suo salotto vive un'ulteriore diaspora.

Tuttavia, nonostante l'aggravarsi della cecità e il dolore per la morte del consorte, la nobildonna fiorentina continua a tenersi aggiornata, mantenendo sempre quella specie di vocazione nell'aiutare i giovani letterati emergenti. Nel 1892, ad esempio, la salonnière si adopera a favore di una giovane poetessa, Ada Negri, cui intende far assegnare il premio Giannina Milli ${ }^{66}$. Per questo, mentre da una parte si informa sulla personalità della maestrina originaria di Lodi, dall'altra sottopone il suo libro di versi, Fatalità, al giudizio di coloro che un tempo avevano frequentato Borgo de'Greci e l'Antella, sollecitandone i giudizi. Anche Fucini, in quanto poeta e narratore, viene chiamato in causa in una lettera dell'ottobre 1892, nella quale Emilia traccia con brevi tocchi la biografia della poetessa suggerendo pure una chiave di lettura per i suoi versi ${ }^{67}$. Anche in altre lettere spedite dalla Peruzzi ai propri conoscenti compare la figura di Ada Negri: vengono utilizzate pressoché le medesime parole per descri-

63 Cfr. la lettera CXXXIX.

64 Si veda la lettera CXLII (6 giugno 1891): «Arrivato a Firenze per venire all'Antella, il Dott. Roster mi dice che gli amici sono pregati a sospendere le loro visite».

65 Cfr. la lettera CXLVI (14 ottobre 1892): «Caro Fucini, tutto quello che è avvenuto da quel giorno avevo la forza di vedere gli amici e il Genala mi disse poi quante affettuose parole le suggerì il suo affetto per il mio Ubaldino e per me».

66 Per aiutare economicamente la poetessa Giannina Milli, Emilia si era fatta promotrice di una sottoscrizione per raccogliere la somma necessaria a garantirle una modesta agiatezza, per poi costituire, alla sua morte, un'istituzione nazionale a lei intitolata (cfr. M.P. Cuccoli, Emilia Toscanelli Peruzzi, cit., p. 202-203). Nel 1894 detto premio venne assegnato ad Ada Negri con la seguente motivazione: «Libro notevole per immediata e gagliarda intuizione del vero e per intima apprensione del sentimento umano: poesia che, nutrita di dolore, sa dagli strazi di questo sollevarsi a idealità più o meno tranquille; ma illuminate dalla fede in un ordine di giustizia superiore e di carità universale» (si veda A. GORINI SANTOLI, Invito alla lettura di Ada Negri, Milano, Mursia, 1995, p. 33). La poetessa rinunciò alla rendita di duemila lire annue in seguito al matrimonio con l'industriale Garlanda, che le assicurò l'agiatezza economica (cfr. M. PeA, Ada Negri, Milano, Mondadori, 1970, p. 75). Non sappiamo chi, dopo la morte di Giannina Milli, abbia fatto il nome della Negri alla Peruzzi (si veda P. RajNA, Emilia Peruzzi e Ada Negri, in «Nuova Antologia", CCXLV (1 gennaio 1926), p. 30).

67 Cfr. le lettere CXLVI e CXLVIII. Emilia si dilunga più diffusamente sulle qualità della Negri in una lettera al giornalista tedesco Sigmund Münz, posteriore di appena due giorni a quella inviata a Fucini: "Ora vi è una nuova poetessa, Ada Negri, che merita un articolo non solo per il suo ingegno, ma anche per la novità degli argomenti ispirati dalle nuove idee e dai tempi nuovi. [...]. È un ingegno virile ed innato, descrisse e cantò quello che vide e che intuì; non è socialista, non è una donna emancipata. Il vigore dei versi di una brava ragazza ventenne sorprende; ma nel "Birichino di strada", nella "Nenia materna", nei versi "Pietà", si sente un cuore gentile e soave di donna". Cfr. S. Münz, Emilia Peruzzi, in «Neue freie Presse», 27 luglio 1900, poi in «La Rassegna Nazionale», CXIX (16 maggio 1901), p. 212. 
vere la misera vita della poetessa e per commentarne i versi. A Carolina Pironti, ad esempio, dopo aver chiesto il giudizio di Matilde Serao e di altri letterati napoletani, scrive piuttosto ingenuamente:

[...] i versi di Ada Negri, ventenne, originali, vigorosi e dolci quando parla di sua madre e della sua infanzia, ispirano grandi ammirazioni ${ }^{68}$.

Fucini legge effettivamente i versi della Negri, o almeno così dice alla sua corrispondente, ma il suo giudizio, per quanto positivo e moderatamente entusiasta, risulta piuttosto scarno ${ }^{69}$, dedicando a Fatalità poche frasi, piuttosto scontate e che ricalcano fedelmente il parere espresso dalla sua corrispondente.

Nel 1895 donna Emilia, sempre più sola nel palazzo avito e abbandonata da molti degli antichi frequentatori del suo salotto, si trasferisce per alcuni mesi a Viareggio $^{70}$. Da qui si congratula con lo scrittore per l'imminente pubblicazione di un "volume preziosissimo» ${ }^{71}$, la raccolta di bozzetti All'aria aperta, di cui fa dono alla signora. Emma Fucini, facendo le veci del marito assente, risponde alla lettera di ringraziamento della Peruzzi con un biglietto di particolare interesse, che illumina un aspetto precedentemente toccato, ossia quello dell'atteggiamento di Fucini in relazione ai propri lettori. Il documento indica una volta di più come egli non fosse del tutto indifferente alla sorte delle proprie opere, ma come, al contrario, vivesse con apprensione la pubblicazione dei propri lavori, preoccupandosi della reazione sia del pubblico che della critica:

Speriamo che il libro (All'aria aperta) incontri <successo> e che Renato calmi la sua agitazione per l'esito che egli teme sempre incerto, come ha temuto per tutti i suoi scritti ${ }^{72}$ !

L'ansia che sembra celare una sostanziale sfiducia in sé stesso e nelle proprie capacità di scrittore va probabilmente collegata al senso di delusione vissuta anni addietro, quando era stato costretto, come già visto, ad abbandonare Firenze per l'impossibilità di trarre proventi sufficienti dalla sua attività letteraria.

Negli anni successivi la corrispondenza si fa sempre più sporadica, in particolare a causa delle condizioni di salute di Emilia. Lo scrittore continua a tenerla al corrente, seppur sommariamente, delle circostanze della sua vita, descrivendole, come da tempo aveva fatto, le visite che il suo impiego lo costringe a compiere e le numerose incombenze che gli derivano dal suo lavoro ${ }^{73}$.

\footnotetext{
68 Si veda P. Rajna, Emilia Peruzzi e Ada Negri, cit., p. 28.

69 Cfr. la lettera CXLIX.

70 Cfr. E. Steinmann, Emilia Peruzzi, cit., p. 220.

71 Si veda la lettera CLXVI.

72 Si veda la lettera CLXVIII.

73 Cfr. la lettera CLXIX.
} 
Nel 1897 Fucini si riporta con la famiglia a Dianella ${ }^{74}$, e questo è l'ultimo spostamento di cui renderà conto all'amica prima della sua morte, avvenuta all'Antella l'8 maggio 1900. Negli ultimi tempi Emilia era riuscita a spedire agli amici, e dunque anche al Fucini, esclusivamente dei biglietti da visita che la segretaria Irene Brunelleschi si incaricava di scrivere in sua vece.

2. È soprattutto dal 1876 che il Fucini si trova costretto sempre più di frequente a pregare l'amica, come egli scrive, di far «scricchiolare [la penna] sopra un pezzetto di carta» per aiutarlo «a rampicare sul calvario del pane quotidiano» ${ }^{75}$, entrando così a far parte della complessa rete di relazioni clientelari messa in piedi dai Peruzzi, una rete ben ramificata e caratterizzata da forte solidarietà ${ }^{76}$.

Donna Emilia si trovava in una posizione strategica all'interno del sistema clientelare, pur non avendo titolo per la distribuzione di favori connessi all'ambito politico ${ }^{77}$. Mentre il marito teneva generalmente un atteggiamento più defilato e meno incline ad accondiscendere alla mole di richieste che pervenivano continuamente all'Antel$\mathrm{la}^{78}$, Emilia appare più sollecita e attenta, manifestando preoccupazione, ad esempio, per la sorte di Fucini e addirittura intervenendo a suo beneficio, prima ancora che questi esprimesse il bisogno di un intervento influente ${ }^{79}$. Il forte senso dell'amicizia spingeva la donna ad andare incontro alle esigenze degli amici, tuttavia il discorso è probabilmente più complesso e chiama in causa anche quel forte legame che una

74 Cfr. la lettera ClXXIII, datata 15 gennaio 1898: «Ed io ho dovuto, fino dal Novembre, abbandonare al buio e alla polvere la mia bella casina di Via Pinti e rintanarmi a Dianella dove la solitudine fa delle brecce desastrose nella serenità del mio spirito».

75 Si veda la lettera LXXI.

76 Sul clientelismo si vedano in particolare Clientelismo e mutamento politico, a cura di L. Graziano, Milano, Angeli, 1974; ID., Clientelismo e sistema politico. Il caso dell'Italia, Milano, Angeli, 1979; E. FrANZINA, Le strutture elementari della clientela in La scienza moderata. Fedele Lampertico e l'Italia liberale, a cura di R. Camurri, Milano, Angeli, 1992, pp. 377-430; L. Musella, Individui, amici, clienti. Relazioni personali e circuiti politici in Italia meridionale tra Otto e Novecento, Bologna, Il Mulino, 1994. Circa il network clientelare dei Peruzzi si veda F. ANDrEUCCI, "Vorrei procacciarmi un'occupazione proficua». Nemesio Fatichi e il clan Peruzzi fra clientelismo, raccomandazioni, politica, in Ubaldino Peruzzi, un protagonista di Firenze capitale, a cura di P. Bagnoli, cit., pp. 145-154.

77 Si veda F. ANDREUCCI, "Vorrei procacciarmi un'occupazione proficua». Nemesio Fatichi e il clan Peruzzi fra clientelismo, raccomandazioni, politica, cit., p. 148.

78 Colpisce, in effetti, la sostanziale "assenza” di Ubaldino: nessuna delle lettere in cui si richiede l'intervento di Peruzzi sono indirizzate direttamente a lui, ma Fucini si rivolge a Emilia, la quale, evidentemente, doveva agire come una sorta di filtro (lo stesso accadrà quando, venuto a mancare Ubaldino, Fucini inizierà a ricorrere all'aiuto di Francesco Genala, conosciuto ai tempi di Borgo de' Greci). Cfr. F. ANDreuCCI, "Vorrei procacciarmi un'occupazione proficua». Nemesio Fatichi e il clan Peruzzi fra clientelismo, raccomandazioni, politica, cit., p. 148.

79 Si veda la lettera CVIII, in cui Fucini immagina il tipo di intervento che la Peruzzi intendeva mettere in atto per fargli ottenere un cambiamento di circondario: «[...] conosco, o almeno credo di conoscere il contenuto di questa sua lettera non ricevuta e supponendo rispondo. Il Cav. Bolasco è provveditore di Firenze; il Cav. Bolasco è da Lei conosciuto; il Cav. Bolasco è quello che può aiutarmi efficacemente a tornare a Firenze; Ella è disposta a parlargli in mio favore». 
salonnière, e in particolare Emilia, instaurava con i giovani cui accordava la propria protezione. Se si era attivata in un'opera di patronage, o meglio, di maternage ${ }^{80}$ per fornire all'allora giovane scrittore un'efficace promozione in grado di permettergli un buon esito letterario, è facile capire come ella non intendesse poi rassegnarsi a perdere influenza sul suo protetto di un tempo. Ricca, intelligente e privilegiata sul piano sociale, ma priva di talenti per realizzarsi personalmente, Emilia aveva trovato nel proprio salon e nell'aiuto che poteva offrire a giovani letterati, uno strumento di affermazione individuale e uno spazio dove esercitare la propria autorità. Ovvio che cercasse di mantenere il proprio prestigio e il proprio ascendente su chi aveva beneficiato (anche quando i giovani, ormai affermatisi, tendevano a rendersi indipendenti) sfruttando, per far ciò, in primo luogo le risorse del marito. Anche Fucini si serve della "dolce influenza»" ${ }^{81}$ della sua amica per far giungere le proprie richieste a Ubaldino, probabilmente con la consapevolezza di stuzzicare la vanità della nobildonna nell'attribuirle un ruolo di primo piano.

Se favorire Fucini per Emilia appare un modo per mantenere viva l'autorità di un tempo non così nel momento in cui questi fa presente alla Peruzzi le necessità di alcuni suoi amici. Significativo di questo atteggiamento è il caso del «carissimo amico» ${ }^{82}$ Eugenio Negri. Lo scrittore cerca di interessare la corrispondente alla sorte di questo suo raccomandato facendo perno sul sentimento d'amicizia che lo lega a Francesco Genala ( Ho il rimorso di averlo [Genala] molestato in questi giorni per favorire un mio carissimo amico, ma egli deve aver quello di non aver risposto a una mia ultima lettera») ${ }^{83}$, un'amicizia nata all'interno del salotto stesso della Peruzzi, che Fucini lusinga sottilmente, quasi invitandola ad assumere un ruolo di garante dell'intimità creatasi fra lui e il politico. E infatti, l'amica fiorentina si dimostra sensibile a questo tipo di sollecitazione, cogliendo l'occasione per riappropriarsi di quell'autorità e di quel prestigio persi in seguito alla morte del marito. Ma non potendo contare sulle amicizie politiche di Ubaldino la situazione non si sblocca e lo scrittore torna alla carica, facendo stavolta leva su tipici sentimenti femminili, quali la compassione, la disponibilità verso il prossimo, e appellandosi al suo senso della giustizia, nel tentativo di impietosire la corrispondente ${ }^{84}$. In questo secondo caso, la Peruzzi tace, e il contenuto di questa perorazione cade nel silenzio, né più vi accenna Fucini, non sappiamo se perché si sia reso conto che a nulla valgono le sue preghiere o se per l'intervento risolutivo di Genala.

È significativo che Fucini, al momento di chiamare in causa Emilia, faccia appello alle doti generalmente richieste ad una donna, come la pazienza, la pietà, la moderazione,

80 Sul ruolo di patronesse assunto dalle donne di ceto alto nel corso dei secoli si veda Ragnatele di rapporti. Patronage e reti di relazione nella storia delle donne, a cura di L. Ferrante, M. Palazzi, G. Pomata, Torino, Rosemberg e Sellier, 1988.

81 Cfr. la lettera CII.

82 Cfr. la lettera CXLVII.

83 Si veda la lettera CXLVII.

84 Si veda la lettera CLII. 
la propensione materna. Abbiamo una lettera del 1896, che ci costringe a considerare almeno di sfuggita il motivo della beneficenza inteso come "vocazione", come "dovere sociale" dei ceti privilegiati e in particolare delle donne ${ }^{85}$ :

E ora, caro Fucini, mi dirigo a Neri Tanfucio chiedendo una poesia da mettersi nel numero unico che sarà stampato qui per il 24 Agosto a benefizio dello Spedale in parte costruito ${ }^{86}$.

La beneficenza, espressione concreta della carità, costituiva per una signora di classe elevata un obbligo a cui non era lecito sottrarsi, anche perché per secoli, l'azione benefica fu una delle poche attività extradomestiche consentite alle donne dei ceti abbienti ove poter avere mansioni e responsabilità di livello istituzionale ${ }^{87}$.

Naturale che anche Fucini fosse costretto a pagare una "contropartita" per gli aiuti ricevuti. La lettera appena presa in considerazione, al di là del fatto che il nostro scrittore avesse o meno aderito alla richiesta, ci fa comprendere quale fosse la tipologia d'intervento comunemente richiesta ad uno scrittore, intervento peraltro difficilmente spendibile da Ubaldino a livello politico, dato che, a quanto risulta dall'intero carteggio, una sola volta Fucini accetta di agire da "galoppino elettorale" per «mandare avanti» ${ }^{88}$ l'amico Vilfredo Pareto $^{89}$, il quale avendo già tentato senza successo l'avventura politica nel 1880, si era poi candidato due anni dopo nel collegio di Pistoia-Prato-San Marcello Pistoiese. ${ }^{90}$ E, stando ad una lettera di Fucini a Emilia ${ }^{91}$, ancora nel 1884, l'economista genovese, determinato a presentarsi alla nuova tornata elettorale, coinvolse l'intero clan Peruzzi. L'autore dei Cento sonetti viene così "ingaggiato" non da Ubaldino in persona, o dalla moglie di lui, ma da uno dei tanti frequentatori dell'illustre salon, fatto questo, che ribadisce l'esistenza di un gruppo piuttosto articolato e coeso facente capo al leader dei moderati toscani ${ }^{92}$.

Comunque, questo è l'unico intervento di tipo esplicitamente politico di cui troviamo traccia nelle lettere di questo carteggio. Per il resto, è soprattutto la Peruzzi a usufruire dei servigi del Fucini, esigendo da lui prestazioni di carattere letterario,

85 Si veda M. T. Mori, Salotti. La sociabilità delle élite nell'Italia dell'Ottocento, cit., p. 160.

86 Si veda la lettera CLXVI.

87 Si veda, L. Ferrante, M. Palazzi, G. Pomata, Introduzione a Ragnatele di rapporti, a cura di Ead., Torino, Rosemberg e Sellier, 1988, p. 16.

88 Cfr. la lettera CXIX.

89 Si veda ancora la lettera CXIX.

90 Circa le disavventure elettorali di Pareto, si veda T. Giacalone Monaco, Introduzione a V. Pareto, Lettere ai Peruzzi, cit., pp. LX-LXIV.

91 Tommaso Giacalone Monaco, nella sua introduzione al carteggio paretiano, non fa riferimento a questa nuova candidatura (cfr. ancora T. Giacalone Monaco, Introduzione a V. PARETo, Lettere ai Peruzzi, cit., pp. LX-LXIV).

92 Cfr. F. Andreucci, "Vorrei procacciarmi un'occupazione proficua». Nemesio Fatichi e il clan Peruzzi fra clientelismo, raccomandazioni, politica, cit., p. 149. 
come la recita di versi, più spesso improvvisati, di solito in occasione della festa di San Carlo $^{93}$. Tuttavia la risposta del poeta è quasi sempre negativa ${ }^{94}$, talché l'opinione di Fucini poeta improvvisatore ${ }^{95}$, diffusa nella critica, in particolare di inizio Novecento, risulterebbe di molto ridimensionata acquistando viceversa ulteriore credito quella del Pancrazi secondo la quale nei suoi versi andrebbe ricercato più di frequente "accorgimento d'arte e disposizione d'effetti, e a tratti direi [...] letteratura» ${ }^{96}$.

Anche l'episodio concernente Ada Negri costituisce un caso evidente in cui Emilia fa pesare i risultati del suo patronage. Fucini in queste occasioni si mostra piuttosto restio a soddisfare le richieste dell'amica, assumendo un atteggiamento che potremmo definire aristocratico, nel senso che non pare disposto a barattare in alcun modo la propria indipendenza di giudizio ritenendo quella dello scrittore un'attività che non può sottostare alle regole del dare e dell'avere caratteristiche dei rapporti clientelari.

3. L'immagine di un Fucini orgoglioso e consapevole del proprio valore di scrittore viene in qualche modo a essere confermata anche da quelle lettere, piuttosto frequenti soprattutto a partire dalla fine degli anni Ottanta, in cui egli si lamenta della mole di lavoro che gli impedisce di dedicarsi liberamente alla letteratura. Sente che l'impiego di ispettore scolastico ( $\mathrm{ma}$, in precedenza, anche quello di insegnante) ${ }^{97}$ lo distrae dall'occupazione sentita come più congeniale, quella dello scrittore, costringendolo a impegnare le sue forze in un'attività "secondaria" senza per questo aver l'impressione di portare un contributo tangibile alle sorti della scuola italiana. Da qui lo sdegno per le $"$ macchinette burocratiche» ${ }^{98}$, sorta di leitmotiv del carteggio ${ }^{99}$.

93 Si veda, a titolo d'esempio, la lettera LXXV.

94 Si vedano, a titolo d'esempio, le seguenti lettere: LXXVI ( DDa questa scelleratissima musa però non è stato possibile avere un pronunciamento carlista»); CLXXXVII ( Passando da Empoli con la mia famiglia per andarmene a Firenze, trovo il suo biglietto dove mi prega di impostare una risposta sonettata per oggi stesso... O come devo fare?»).

95 Si vedano ad esempio: B. Croce, Renato Fucini, cit., passim; F. PAOLIERI, Renato Fucini umorista, in "La Nazione», 18 giugno 1913; M. TAdDEI, Renato Fucini, in "Nuova Antologia», CCXLV (1 e 16 gennaio 1926), p.65; P. Bargellini, A veglia con Renato Fucini, cit., p. 20; E. Lama, Giudizio su Renato Fucini, cit., p. 8.

96 Cfr. P. Pancrazi, Renato Fucini, in ID., Venti uomini, un satiro e un burattino, Firenze, Vallecchi, 1923, p. 106.

97 Cfr. la lettera LXXXIX: «Ogni mattina mi alzo alle 6, corro alla stazione e di lì a Pistoia dove appena arrivato, senza un respiro, debbo fare tutti i giorni tre e a volte quattro ore di lezione a 150 speranze d'Italia divise in tre classi; appena ho finito, di corsa al vapore e via a Firenze. Arrivato a Firenze alle 2 ho da studiare, da prepararmi alle lezioni del giorno dopo, ho da riguardare componimenti, ho da arrabattarmi, in somma, come un cane [...]. Pensi a molte altre preoccupazioni che debbono molestarmi in un momento così critico; pensi che dopo tanta fatica di muscoli e di mente, l'orizzonte che mi si presenta la sera è quello di riprincipiare il giorno dopo alle sei [...]».

98 Si veda la lettera CXLVII.

99 Si vedano in particolare le lettere CXIV, CXXXVIII, CXLVII, CLII, CLIV, CXLIV, CXLIX. 
Fucini, privo di una rendita patrimoniale capace di assicurargli l'agiatezza ${ }^{100}$, vive a pieno la cosiddetta "perdita d'aureola", benché non ne sembri del tutto consapevole e non giunga mai, per quanto è possibile comprendere dalle sue lettere e dalla sua opera, a una riflessione critica, cui invece approdano, un po' in tutta Europa, molti altri intellettuali. Questo è tanto vero da riverberarsi anche su tono e contenuti della sua scrittura che, rispetto al tono burlesco dei Cento sonetti, sembra essere pervasa da un sentimento di amarezza e dove la spontanea ilarità dei sonetti lascia il posto alla descrizione frequente del dolore e della miseria ${ }^{101}$. Quest'atmosfera di malinconia fa capolino anche nelle lettere, dove lo scrittore proietta un'immagine di sé decisamente diversa da quella trasmessaci da certa critica, cioè di un Fucini "buontempone" e spensierato, sempre pronto alla burla. In realtà, qui viene tratteggiato un ritratto che sembra coincidere con quello lasciatoci da altri studiosi, come per esempio il Biagi, secondo il quale l'ilarità dello scrittore sarebbe soltanto un atteggiamento di facciata e velerebbe una "primigenia tristezza interiore» ${ }^{102}$.

Sempre da queste lettere è possibile intuire come il lavoro di ispettore scolastico, oltre a costringerlo lontano da una grande città e dalle opportunità che essa avrebbe potuto offrigli, lo impegna a tal punto da impedirgli quasi completamente di scrivere. Annichilito dalle inutili scartoffie da compilare («[...] ora mi par d'essere diventato uno scagnozzo arrapinato, con l'aggravante dello sconforto che mi piglia quando vedo che, gira e rigira, le cose dell' istruzione vanno oggi, dopo tanto lavorio, come andavano press'a poco quando ero canonico gaudente» ${ }^{103}$ ), Fucini si trova a dover rifiutare offerte che gli giungono da diverse parti per mancanza di tempo ${ }^{104}$.

Si delinea dunque una situazione di gran lunga diversa da quella generalmente presentata dalla critica, volta a sostenere l'esistenza di un "periodo d'oro" apertosi per Fucini nel 1879, con la nomina a ispettore scolastico. Se non si può mettere in dubbio

100 Fucini, in realtà, aveva dei possedimenti nei dintorni di Empoli, che però non gli fornivano proventi bastanti a condurre un vita agiata (si veda la lettera CXLVII: "[...] son costretto a far l'impiegato per supplire col mio lavoro a quello che il mio piccolo patrimonio non mi dà»). Che lo scritore rimpiangesse di non avere una rendita tale da assicurargli una comoda sussistenza è testimoniato da alcune lettere: CXLVII («Non vedo più nulla, non so più nulla e quasi non leggo più nulla per la vita che meno e per le persone in mezzo alle quali la meno. Ah mio Dio! Se il compianto Livingstone si fosse, insieme coi vetturini di Firenze e con l'Av. Barsanti, ricordato anche di me, non parlerei ora a questa maniera»); CLXII («E siccome cartelle con premio non ne posseggo, al lotto non gioco, eredità alle viste non ce ne ho punte, così mi son rassegnato e tiro avanti come quel somaro filosofo, il quale, girando il bindolo, trovava una gran consolazione a pensare ai cavalli degli omnibus»).

101 Su questo aspetto della prosa fuciniana si veda in particolare L. Russo, Fucini scrittore sollazzevole?, in ID., Ritratti e disegni storici, serie IV, Dal Manzoni al De Sanctis e la letteratura dell'Italia unita, Firenze, Sansoni, 1965, pp. 339-343.

102 Si veda G. BIAGI, Renato Fucini nella vita e nell'arte, cit., pp. 74-75.

103 Cfr. la lettera CXLVII.

104 Cfr. la lettera CLII. Si veda anche la lettera inviata a Giuseppe Chiarini per rifiutare la una proposta di collaborazione alla «Domenica del Fracassa»: «Due terzi dell'anno sono a giro per questi monti, digrumatore eterno delle mie dieci idee, l'altro terzo me lo bestemmio a tavolino con la legge Casati da una parte, nel mezzo le mie coglionerie e da quell'altra quelle dei Sindaci del Circondario. Creda è un miracolo se piglio palla» (cfr. A. CHITI, Ricordi pistoiesi di Renato Fucini, cit., p. 20). 
che gli anni d'ispettorato siano risultati essere fondamentali per la sua formazione artistica, è altresì innegabile che lo scrittore avverta come degradanti le attività quotidiane che lo distraggono dal suo vero "mestiere", quello dello scrittore appunto, mentre si sente abbrutito dalle sue mansioni di dipendente dello Stato.

Ma se Fucini cerca di suscitare la simpatia e la comprensione della sua corrispondente, tracciandole la difficile situazione in cui si trova, stretto tra la necessità di lavorare e il desiderio di scrivere, Emilia appare piuttosto distratta e poco convinta di fronte alle sue continue lamentele ${ }^{105}$. Come abbiamo già avuto modo di segnalare, tra i due protagonisti del carteggio si può notare una certa discordanza anche su argomenti di un certo peso. È possibile ipotizzare che Fucini si sentisse scarsamente compreso dall'amica fiorentina, che tende spesso a ridimensionare l'importanza dei problemi che le vengono sottoposti. 


\section{NOTA AL TESTO}

Il carteggio tra Renato Fucini ed Emilia Peruzzi abbraccia un arco di tempo assai ampio (circa trent'anni), ed è interrotto solo dalla morte della nobildonna fiorentina, avvenuta nel maggio del 1900 (l'ultima lettera, scritta da Fucini, è datata 1 gennaio 1899). Se la Peruzzi ha serbato la totalità (o quasi) delle missive inviategli dallo scrittore, dobbiamo segnalare la mancata conservazione, da parte di questi, di molte lettere della corrispondente. Per l'intero periodo dell'epistolario, a fronte di 151 lettere di Fucini, ne restano solo 41 di Emilia. A questo dobbiamo aggiungere la scarsità di sue lettere relative agli anni iniziali della corrispondenza. Per quanto riguarda lo scrittore, i primi anni del carteggio sono ricchi di biglietti da visita scritti pressoché quotidianamente; nel prosieguo della raccolta, e soprattutto dagli anni Ottanta, si assiste a una progressiva diminuzione del numero delle lettere inviate alla corrispondente, situazione che si aggrava ulteriormente con gli anni Novanta. Nel periodo che va dal 1871 al 1877, poi, i brevi biglietti sono spesso privi di data autografa. Gli originali fuciniani sono stati datati in un tempo immediatamente precedente al loro ingresso nella Biblioteca Nazionale Centrale (dove sono tuttora conservati), probabilmente dagli esecutori testamentari, in base ad annotazioni riguardanti giorno, mese e anno d'arrivo, che la signora Peruzzi era solita apporre sulle buste ${ }^{106}$. Lo stesso vale anche per gli autografi del Fondo Fucini presenti nella Biblioteca Riccardiana di Firenze; in questo caso, tuttavia, siamo costretti a segnalare come le date, spesso, siano state erroneamente attribuite, rendendo ovviamente inutilizzabili tutte le indicazioni di questo tipo ${ }^{107}$.

L'edizione è stata condotta sui manoscritti originali, conservati rispettivamente, come già detto, nella Biblioteca Nazionale Centrale di Firenze (Carte Emilia Peruzzi) e nella Biblioteca Riccardiana della medesima città (Fondo Renato Fucini). Le lettere (I-CLXXVI) sono state disposte in ordine cronologico; quelle di data incerta o sconosciuta (CLXXVII-CXCII) sono state poste in appendice. Alcune lettere della Peruzzi (XIII; LXXVIII; LXXX; LXXXIII; LXXXVII; XCVI; CIII; CXXXV; CLXVII; CLXXXIX; CXC) sono in realtà scritte su fogli dalle dimensioni molto ridotte; in altre (LXXIV; LXXVIII; CII; CXXI; CXLIII) le frasi finali della missiva sono state sovrapposte a quelle della prima facciata.

106 Solo una minima parte delle buste originali sono state conservate dagli esecutori testamentari; in particolare, sono giunte fino a noi solo quelle relative alle lettere scritte negli anni Novanta.

107 Per quanto riguarda gli autografi di Fucini, si sono rinvenuti solo due errori di datazione. 
In calce ad ogni lettera sono segnalate, nell'ordine: luogo di conservazione, segnatura, descrizione sintetica del documento, numero delle facciate e delle carte, informazioni relative alla datazione (se congetturale), eventuale precedente pubblicazione. Ogni lettera presenta un numero progressivo relativo alla successione dei documenti, seguito dal mittente e dal destinatario.

ABBREVIAZIONI E SEGNI TIPOGRAFICI CONVENZIONALI:

BNCF: Biblioteca Nazionale Centrale di Firenze

BRF: $\quad$ Biblioteca Riccardiana di Firenze

CEP: $\quad$ Carte Emilia Peruzzi

FRF: $\quad$ Fondo Renato Fucini

Aut.: $\quad$ autografo

Cass.: $\quad$ cassetta

Ins.: $\quad$ inserto

Bigl. vis.: $\quad$ biglietto da visita

Cart. Post.: cartolina postale

Ind.: $\quad$ indirizzo

Ann.: $\quad$ annotazione

$<\quad \quad$ indicano integrazione congetturale di parole o lettere mancanti per svista d'autore;

[*] indica parola indecifrabile;

[?] indica che la lezione immediatamente precedente non è sicura per difficoltà di lettura;

Corsivo: indica che il testo è sottolineato una volta;

Grassetto: indica che il testo è sottolineato due volte. 


\section{CARTEGGIO}

RENATO FUCINI - EMILIA PERUZZI

(1871-1899) 

R. FuCini a E. PERUZZI

Sono dispiacentissimo, ma un impegno contratto prima che mi giungesse il di lei gentile biglietto, mi impedisce di venire Domenica all'Antella ${ }^{1}$.

Tanti dei soliti complimenti.

6 Luglio 1871

\section{II}

R. Fucini a E. PeruZzi

Sono mortificato da tanta gentilezza e mi dispiace di non poter accettare nemmeno per Domenica prossima il di lei invito. La mia povera $\operatorname{moglie}^{1}$ non sta punto bene in salute e la

I. Dall'aut. in BNCF (CEP, cass. 74, ins. 8, n. 10). Bigl. vis. adespota intestato "ING. ReNATO FuCINI". Inedito.

1. All'Antella, località nei pressi di Firenze, sorge la villa patrizia dei Peruzzi, dove Ubaldino ed Emilia trascorrevano i mesi più caldi dell'anno. Vi si ritirarono definitivamente in seguito alla bancarotta del Comune di Firenze (1876), per far fronte alla quale Peruzzi perse gran parte dei propri beni. Dopo la morte della signora Emilia, venne venduta al figlio di Robert Browning e Elizabeth Barret. Si veda M. Casprini, Villa La Torre-Peruzzi, in U. Bardi, M. Casprini, M. Turchi, I Peruzzi all'Antella, Antella, C.R.C. Antella, 1998, pp. 6-7.

II. Dall'aut. in BNCF (CEP, cass. 74, ins. 8, n. 11). Bigl. vis. adespota intestato "ING. RenATO FuCINI". Inedito.

1. Si tratta di Emma Roster. Fucini la sposò nel 1867, a ventiquattro anni (cfr. A. NicColAI, Renato Fucini. Saggio biografico critico, cit., p.34). Si veda R. Fucini, Foglie al vento, in ID., Tutti gli scritti, cit., p. 691: «Don Giuseppe Barzacchini, fu, nel suo esilio, il primo maestro di una bambina che si chiamava Emma Roster, la quale diventò poi la buona, la virtuosa, la fedele compagna della mia vita». 
Domenica è per me un giorno prezioso per andarla a trovare in campagna ${ }^{2}$. Il 22 corrente parto per i bagni ma in questa settimana l' egregio Neri saprà trovare il tempo per venire a salutarla. Una miriade di ringraziamenti e di saluti.

Mercoledì 12 Luglio 1871

III

R. Fucini a E. PeruzZi

Un impegno precedentemente contratto mi impedisce di accettare il gentile invito. Ne sono dispiacentissimo, ma spero che ella vorrà scusarmi.

Tanti rispettosi complimenti.

11 Ottobre 1871

\section{IV}

R. FuCINI A E. PERUZZI

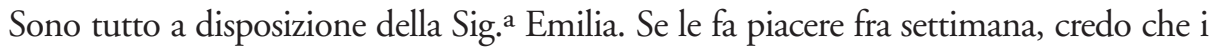
miei superiori non vorranno, per un giorno, negarmi di escire dall'ufficio un ${ }^{1}$ ora avanti ${ }^{2}$. Tanti complimenti e grazie

(Lunedì sera)

2. È probabile che la moglie si trovasse nella villa di proprietà della famiglia Fucini situata a Dianella, nei pressi di Empoli.

III. Dall'aut. in BNCF (CEP, cass. 74, ins. 8, n. 12). Bigl. vis. adespota intestato "ING. RenATO FuCINI". Inedito.

IV. Dall'aut. in BNCF (CEP, cass. 74, ins. 8, n. 13). Bigl. vis. adespota intestato "ING. ReNATO FUCINI". Inedito. Data, non aut. e a lapis: 16 ott. 1871.

1. Così nell'originale.

2. Fin dal 1865 Fucini era impiegato come ingegnere presso il Comune di Firenze. Cfr. L. G. SBrocCHI, Renato Fucini. L'uomo e l'opera, cit., pp. 22-24. 


\section{E. PeruzZi a R. Fucini}

L'imperatore appena arrivato domandò di lei a Ubaldino esprimendo il desiderio di vederla ${ }^{1}$. Tiri dunque fuori la giubba e a domani sera al circolo ${ }^{2}$. Ha ricevuto l'invito? Non ne dubito. Ubaldino la prega di non mancare. Mille cose.

VI

\section{R. Fucini a E. Peruzzi}

Un saluto alla Sig. ${ }^{a}$ Emilia.

\section{R. Fucini a E. PeruZzi}

Ho la moglie incomodata ed è in letto; se stasera starà meglio, come spero, verrò con tutto il piacere.

V. Dall'aut. in BRF (FRF, cass.8, ins. 21). Bigl. vis. adespota intestato "Ubaldino Ed Emilia PeRUZZI”. Scritto probabilmente il 2 dicembre 1871, poiché l'imperatore del Brasile, giunto a Firenze il 30 novembre e ripartito il 5 dicembre, prese parte a una conversazione in Palazzo Vecchio il 3 dicembre (cfr. «La Nazione» dal 1 al 6 dicembre 1871).

1. Si tratta di don Pedro d'Alcantara (Rio de Janeiro 1825-Parigi 1891). Si veda R. Fucini, Acqua passata, cit., pp. 520-522: «Il Peruzzi mi presentò subito a don Pedro il quale pareva che mi aspettasse, e attaccammo subito una animata conversazione in lingua italiana che egli parlava molto bene e senza fatica. Di che parlammo? Mah! Non mi ricordo di nulla». Ubaldino è, ovviamente, il marito della Peruzzi (su di lui si veda Jarro [G. Piccini], Vita di Ubaldino Peruzzi scritta e pubblicata da lui vivente, Firenze, Bemporad, 1891).

2. È il Circolo Filologico, costituitosi il 9 gennaio 1872, con sede in palazzo Spini Ferroni. Ne era presidente Ubaldino Peruzzi. Cfr. V. VannuccI, Istituzioni fiorentine. Raccolta di monografie dei principali Istituti di Beneficenza, Letterari, Scientifici, Educativi, Circoli di ricreazione, ecc..., Firenze, Lumachi, 1902, pp. 289-297. Si veda inoltre R. Romanelli, Il casino, l'accademia e il circolo. Forme e tendenze dell'associazionismo d'élite nella Firenze dell'Ottocento, in Fra storia e storiografia. Scritti in onore di Pasquale Villani, a cura di P. Macry, A. Massacra, Bologna, Il Mulino, 1994, pp. 836-838.

VI. Dall'aut. in BNCF (CEP, cass. 74, ins. 8, n. 14). Bigl. vis. adespota intestato "ING. RenATO FuCINI". Inedito. Data, non aut. e a lapis: 7.XII.71.

VII. Dall'aut. in BNCF (CEP, cass. 74, ins. 8, n. 15). Bigl. vis. adespota intestato "ING. ReNATO FuCINI". Inedito. Data, non aut. e a lapis: 17.1.72. 


\section{VIII}

R. Fucini a E. Peruzzi

Per affari della massima importanza, domani debbo recarmi a Empoli, per conseguenza non potrò venire a trovarla.

Se non fosse indiscretezza le domanderei il favore di mettere in una busta il biglietto che ella tanto gentilmente mi ha offerto e mandarmelo all'Ufficio ${ }^{1}$.

Suo devoto

R. Fucini

23 Gennaio 1872

\section{IX}

\section{R. Fucini a E. PERUZZi}

Solo persone di famiglia alla mia tavola per festeggiare questa tradizionale solennità, per cui oggi non potrei voltare le spalle alla Zuppa domestica senza tirarmi addosso la scomunica de' miei cari ${ }^{1}$.

Il Giacomelli non vorrà attribuire a indifferenza questa mia mancanza, giacché, se la Sig. ${ }^{a}$ Emilia me lo permette, verrei alle 7 per salutarlo ${ }^{2}$.

La ringrazio tanto tanto.

VIII. Dall'aut. in BNCF (CEP, cass. 74, ins. 8, n.16). Bigl. vis. intestato "ING. RenATO FuCINI". Inedito.

1.Era consuetudine della Peruzzi offrire agli amici biglietti gratuiti per spettacoli teatrali o concerti. Cfr. G. Rossi, Salotti letterari in Toscana. I tempi, l'ambiente, i personaggi, cit., p. 114.

IX. Dall'aut. in BNCF (CEP, cass. 74, ins. 8, n. 17). Bigl. vis. adespota intestato "ING. ReNATO FUCINI". Inedito. Data, non aut. e a lapis: 8.2.72.

1. Non sappiamo a quale solennità Fucini si riferisca.

2. Giovan Battista Giacomelli (Livorno 1814-1876). Studiò medicina all'Università di Pisa. Nel 1848, mentre si trovava a Pisa, collaborò col giornale politico fondato da Montanelli, «Italia». Compì vari viaggi (Brasile, Francia, Germania), prima di ottenere un posto di addetto all'ufficio della stampa al Ministero dell'Interno. Seguì la capitale a Firenze, dove iniziò a frequentare i salotti più in vista della città, tra i quali quello di Borgo de' Greci, dove recitava in maniera brillante dapprima i versi di Belli e di Porta, in seguito quelli di Fucini. Su Giacomelli e sulla sua amicizia con Emilia Peruzzi si veda L. M. Fortunato De Lisle, The circle of Pear; Emilia Peruzzi and her salon - political and cultural reflections, issues and exchange of ideas in the new Italy. 1860-1880, cit., pp. 259-272. 


\section{$\mathrm{X}$}

R. Fucini a E. PERUZZi

Domani sera (Lunedì) alle 6 non mancherò. Tanti de' soliti complimenti e mille grazie.

Domenica

R. Fucini a E. Peruzzi

Firenze 6 Marzo 1872

Grazie! Grazie! Grazie!

Sono lontanissimo dal desiderare che mi si presenti questa occasione, ma se vedessi

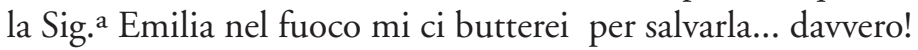

\section{XII}

R. Fucini a E. PeruZzi

Ecco servita fra i primissimi la Sig. ${ }^{a}$ Emilia. Sabato sera verrò a salutarla; parleremo del mio trepidante volumino ${ }^{1}$.

X. Dall'aut. in BNCF (CEP, cass. 74, ins. 8, n. 18). Bigl. vis. adespota intestato "ING. RenATO FuCINI". Inedito. Data, non aut. e a lapis: 11.2.72.

XI. Dall'aut. in BNCF (CEP, cass. 74, ins. 8, n. 19). Bigl. vis. adespota intestato "ING. ReNATO FuCINI". Inedito.

XII. Dall'aut. in BNCF (CEP, cass. 74, ins. 8, n. 20). Bigl. vis. adespota intestato "ING. Renato FucINI". Inedito. Data, non aut. e a lapis: 7.3.72.

1. Si tratta dei Cento sonetti in vernacolo pisano (Firenze, Pellas, 1872), uscito in questo torno di tempo. 
XIII

E. PeruZZI a R. FuCINI

13 Marzo 72

Aggiunga alla sua collezione l'articolino dello Zanella1. A me scrive così "Domani darò qualche riga al giornale di Padova sul bellissimo libro del Fucini ${ }^{2}$. Intanto gli faccia le mie congratulazioni."

Io poi amico La prego notizie quali bozzetti cita lo Zanella - son fra quelli che tutti possono leggere con profitto ${ }^{3}$.

\section{XIV}

R. Fucini a E. Peruzzi

\section{Gentilissima Signora Emilia}

Grazie tante del giornale di Pisa. L'articolo l'ho trovato serio molto e molto ben fatto. Sarei curioso di conoscerne l'autore ${ }^{1}$. E del Roster ha sentito eh ${ }^{2}$ ? Che caro ra-

XIII. Dall'aut. in BRF (FRF, cass. 8, ins. 19). Lettera adespota intestata "EP”, 1 f. su 2 cc. Inedita.

1. Arnaldo Zanella (Monticelli d'Ongina [Piacenza] 1860 - ?). Compì gli studi letterari ed insegnò alla scuola tecnica di Loano, collaborando contemporaneamente a giornali e riviste.

2. Si tratta di una recensione ai Cento sonetti in vernacolo pisano, pubblicata su «L'eco dei giovani», I, marzo-aprile 1872, 2, p. 227.

3. Un sonetto di Fucini venne pubblicato sulla rivista padovana «L'eco dei giovani», II, $1^{\circ}$ ottobre 1873,5 , p. 235. Si tratta del componimento Si fa quel che si pole: «Mi chiamarono al tocco: io mi destai / $\mathrm{E}$ in fretta e ' $\mathrm{n}$ furia mi messi un vestito. / Come tremavo! A un tratto: - O dove vai? - / Mi domandò tra 'l sono 'I mi' marito. / - More - dissi - Ma chi? - More Amaddio - / Fece un salto dal letto, e - Vengo anch'io. - // Quando ripenso a cotesta nottata... / Guardi, signora, ho sempre gli occhi rossi: / Che strazio! Che famiglia desolata! / Io non sapevo più dove mi fossi. / Lei svenuta... d'intorno i suoi bambini... / Cinque che urlavan - mamma - poverini! // Alle quattro spirò - Povero vecchio! / Stette in sé fino all'ultimo momento. / E ogni tanto ammiccava qui all'orecchio / Come volesse dire: - Anch'io li sento!... - / Poi guardava in quell'uscio, fisso fisso, / E piangeva, e baciava il Crocifisso. // Lei da quel giorno, è sempre allo Spedale, / E morirà di certo. La vedesse! / Uno scheletro, un'ombra tal' e quale. / Ché se un pensiero non la trattenesse, / Forse a quest'ora... Oh, sì, lo creda pure, / Campa per quelle cinque creature. // Quell'innocenti si son presi noi. / Gigi era tanto amico d'Amaddio, / Che se li tiene come fosser suoi / E, non lo nego, fo altrettanto anch'io... / De' mezzi non se n'ha, ma cosa vole? / Signora mia, si fa quel che si pole» (cfr. R. FuCINI, Guazzabuglio, in ID., Tutti gli scritti, cit., pp. 977-978).

XIV. Dall'aut. in BNCF (CEP, cass. 74, ins. 8, n. 21). Bigl. vis. adespota intestato "ING. RenATO FuCINI". Inedito. Data, non aut. e a lapis: 29.III.72.

1. È stato impossibile individuare l'articolo, l'autore, nonché il periodico pisano in questione.

2. Giorgio Roster (1843-1927). Professore d'igiene all'Istituto di Scienze Sociali di Firenze e alla Facoltà di Farmacia, s'interessò a molte altre attività scientifiche, dedicandosi allo studio della 
gazzo è quello! Non v'è caso che lui dica mai una cosa per un'altra. Nemmeno l'altra sera quando io ero incomodato.

Dunque a sabato e mille grazie

\section{$\mathrm{XV}$}

R. FuCini a E. PERUZZI

A pranzo è impossibile. Sono impegnato con la famiglia Roster che festeggia coi parenti il promesso matrimonio del figlio.

Intanto, la prego, stringa sodo sodo la mano al Giacomelli per me, e con tanti ringraziamenti arrivederci a stasera ${ }^{1}$.

1 Aprile 1872

\section{XVI}

\section{R. Fucini a E. PeruZzi}

Dovendo stare qualche sera senza venire a complimentare la Sig. Emilia, mi sento in dovere di dirgliene le ragioni, che sono piuttosto dolorose. Stasera avevo divisato di venire al Niccolini ${ }^{1}$ per sentire i Rusteghi di Goldoni, quando una lettera di casa mia mi è giunta annunziandomi che mio Padre $^{2}$ è in letto con un forte attacco di fegato, il qual viscere da tanti anni lo molesta, per cui domani sera anderò a Dianella per trattenermi

mineralogia, della sabbia, dei venti, della pioggia, con particolare riferimento all'isola d'Elba. Si appassionò anche a problemi di erbicoltura e floricoltura. Nel 1871 fu eletto socio dell'Accademia dei Georgofili. Si tratta del fratello della moglie di Fucini, Emma, come risulta chiaro da una lettera di Ottaviano Del Corona, amico di Fucini e frequentatore del salotto Peruzzi, a Emilia (CEP, cass. 56, ins. 11, n. 2): «[...] [Fucini] ha voluto però prendersi la bega di rintracciare per me il suo Cognato Giorgio $[\ldots]$ ».

XV. Dall'aut. in BNCF (CEP, cass. 74, ins. 9, n. 1). Bigl. vis. adespota intestato "ING. Renato FuCINI". Inedito.

1. Su Giovan Battista Giacomelli si veda la nota 2 alla lettera IX.

XVI. Dall'aut. in BNCF (CEP, cass. 74, ins. 9, n. 2). Bigl. vis. adespota intestato "ING. RenATO FuCINI". Data, non aut. e a penna: 12.4.72. Inedito.

1. Il noto teatro fiorentino posto in via Ricasoli.

2. David Fucini, nato a Dianella nel 1816, dove morì nel 1900. Si laureò in medicina a Pisa nel 1842; fervente patriota, nel 1849 perse l'impiego di medico. Uomo eccentrico e originale, compose salaci epigrammi (cfr. R. FUCINI, Acqua passata, cit., pp. 488-491). Si vedano inoltre: A. Zanella, Renato Fucini. Studio biografico critico, Firenze, Bemporad, 1907, pp. 12-15; ID., Il padre di Renato Fucini, in "Il Marzocco», 13 aprile 1913; L.G. SвROcchI, Renato Fucini. L'uomo e l'opera, cit., pp. 11-12. 
la Domenica. Lunedì sera poi non potrò farmi vedere perché ho già ricevuto l'invito del Colonnello per una guardia di gastigo in conseguenza della mancanza commessa l'altra sera ai Pitti e della quale tenemmo parola ${ }^{3}$.

La saluto distintamente

\section{Venerdì}

\section{XVII}

\section{R. FuCini a E. PeruZZI}

Da quattro giorni sono in letto con la Rosolia. Che consolazione! la rosolia a 29 anni. Trovai mio Padre assai bene ma molto deperito per aver passato molti giorni in letto.

Nella supposizione assai fondata di dover passare tutta la settimana in casa, ne avverto tutti gli amici perché non credano ch'io mi sia reso prezioso.

E dell'articolo della Gazzetta d'Italia che gliene pare ${ }^{1}$ ?

Gradisca tanti complimenti da farne parte anche al Sig Ubaldino.

Mercoledì 17 Aprile 1872

\section{XVIII}

\section{R. Fucini a E. PeruzZi}

Ieri l'altro fui in guardia di gastigo ai Pitti. Stasera vado ad accompagnare la mia famiglia in campagna. Ecco giustificata la mia assenza di ieri l'altro e di stasera. Nella settimana ventura, il povero fabbricante di sonetti, verrà a complimentare la Signora Emilia.

4 Maggio 1872

3. Fucini fece parte della Guardia Nazionale Fiorentina arrivando a ricoprire la carica di caporale (cfr. R. FucINI, Acqua passata, cit., pp. 505-510). Sulla Guardia Nazionale, nata per espletare servizio di guardia all'interno del comune o della città di appartenenza tramite il presidio degli edifici pubblici e la repressione dei tumulti, si veda S. Ales, La Guardia Nazionale italiana. 1861-1876, Roma, Ufficio storico SME, 1994.

XVII. Dall'aut. in BNCF (CEP, cass. 74, ins. 9, n. 3). Bigl. vis. adespota intestato "ING. RenATO FuCINI". Inedito.

1. Si tratta di una recensione ai Cento sonetti in vernacolo pisano (Firenze, Pellas, 1872), dove Fucini viene definito poeta popolare, che, attraverso la spontaneità dei suoi versi, riesce a cogliere l'animo del popolo e la sua lingua. Cfr. G.O., Renato Fucini, in «Gazzetta d'Italia», 14 aprile 1872.

XVIII. Dall'aut. in BNCF (CEP, cass. 74, ins. 9, n. 5). Bigl. vis. adespota intestato "ING. ReNATO FuCINI". Inedito. 


\title{
XIX
}

\author{
R. Fucini a E. PeruZzi
}

Prima di partire per la campagna, dove mi tratterrò niente meno che due giorni,

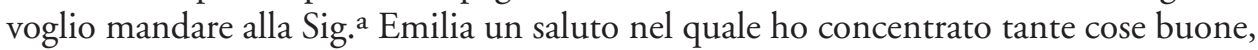
da confondere tutti coloro che mi vogliono un pessimista arrabbiato.

Firenze 18 Maggio 1872

\section{XX}

R. FuCini a E. PERUZZI

Grazie del saluto ricambiato; grazie del bigliettino a me e grazie di quello al DelCorona nel quale ha voluto rammentargli del Fucini ${ }^{1}$.

Le Domeniche, ormai, le ho tutte impegnate co' miei Genitori ${ }^{2}$ e con la mia cara famigliola, di modo che, per gli amici, non mi restano che i giorni feriali e qualche festicciola fra settimana, come sarebbe Giovedì venturo nel qual giorno mi troverò in disponibilità, ma non ne sono certo.

In ogni modo le sono gratissimo del desiderio che Ella mostra di rivedermi, e le assicuro che io sono dolentissimo di non poter complimentare tanto spesso quanto vorrei la gentilissima Sig. ${ }^{\text {a Emilia. }}$

Un mirallegro di cuore per la splendida vittoria riportata ultimamente dal sig. Ubaldino alla Camera.

25 Maggio 1872

XIX. Dall'aut. in BNCF (CEP, cass. 74, ins. 9, n. 6). Bigl. vis. adespota intestato "ING. RenATO FucINI". Inedito.

XX. Dall'aut. in BNCF (CEP, cass. 74, ins. 9, n. 7). Bigl. vis. adespota intestato "ING. Renato FuCINI". Inedito.

1. Ottaviano Del Corona fu, con ogni probabilità, collega di Fucini quando questi era impiegato del Comune di Firenze (cfr. l'aut. in BNCF, CEP, cass. 56, ins. 11, n. 11, lettera datata 15 ottobre 1875: "[...] come purtroppo si avverte anche in Uffizio la sua [di Fucini] mancanza [...]»). In seguito passò a lavorare per la Società delle Ferrovie Meridionali (cfr. l'aut. in BNCF, CEP, cass. 56, ins. 11, n. 20, lettera datata 20 agosto 1891: «Tanto volentieri accetterei il confidenziale invito fattomi di una colazione all'Antella ma è stato soppresso quà [sic] alle Meridionali il turno festivo già da anni, e quindi bisognerebbe chiedere un permesso speciale»).

2. David Fucini (si veda la nota 2 alla lettera XVI), e Giovanna Nardi, nata a Livorno nel 1820. 
XXI

R. FuCini a E. PeruZZi

Grazie dell'invito, e prometto di non mancare, a meno che qualche caso straordinario non me lo impedisca.

Ho veduto il Roster il quale mi ha detto che partirà alla metà del prossimo Giugno e che è tutto lieto di potere un giorno, prima della sua partenza, presentare i suoi omaggi alla Sig. ${ }^{a}$ Emilia ${ }^{1}$. Il Del-Corona si è entusiasmato seriamente delle amichevoli parole di conforto scrittegli ultimamente da Lei.

La saluto distintamente.

Martedì 28 Maggio 1872

\section{XXII}

R. Fucini a U. PeruZZI

Stimato Sig. Ubaldino

Secondo quello che mi dice la Sig. ${ }^{\text {a }}$ Emilia l'avverto che stasera verrò all'Antella in compagnia del dott. Roster.

\section{Suo devoto}

R. Fucini

Giovedì 6 Giugno 1872

XXI. Dall'aut. in BNCF (CEP, cass. 74, ins. 9, n. 8). Bigl. vis. adespota intestato "ING. RenATO FuCINI". Inedito.

1. Su Giorgio Roster si veda la nota 2 alla lettera XIV.

XXII. Dall'aut. in BNCF (CEP, cass. 74, ins. 9, n. 9). Bigl. vis. intestato "ING. Renato FuCinI". In alto a dx ann. non aut.: «Ubald». Inedito. 


\title{
XXIII
}

\author{
R. Fucini a E. PeruzZi
}

Per dire il vero, d'ora in avanti il Fucini si trova mortificato di fronte a tante gentilissime e ripetute testimonianze di simpatia, ma siccome un invito della Sig. ${ }^{a}$ Emilia vale per me un dolce comando, verrò. Intanto le anticipo i miei ringraziamenti e le proteste della mia più sentita stima ed amicizia.

- Venerdì -

\section{XXIV}

R. Fucini a E. PeruZzi

Non ho risposto subito al biglietto d'invito per vedere se m'era possibile sistemare le mie cose in modo da esser libero domani. Non è stato possibile!

La ringrazio di tutto cuore e a quest'altra volta.

- Sabato.-

\section{XXV}

\section{R. Fucini a E. PeruZZI}

Accetto con tanto piacere e la ringrazio moltissimo.

La prego de' miei rispettosi complimenti pel Sig. Ubaldino.

3 Luglio 1872

XXIII. Dall'aut. in BNCF (CEP, cass. 74, ins. 9, n. 10). Bigl. vis. adespota intestato "ING. RenATO FucINI". Inedito. Data, non aut. e a lapis: 14.6.72.

XXIV. Dall'aut. in BNCF (CEP, cass. 74, ins. 9, n. 11). Bigl. vis. adespota intestato "InG. Renato FuCINI". Inedito. Data, non aut. e a lapis: 22.VI.72.

XXV. Dall'aut. in BNCF (CEP, cass. 74, ins. 9, n. 12). Bigl. vis. adespota intestato "ING. Renato FucINI". Inedito. 


\title{
XXVI
}

\author{
R. Fucini a E. PeruzZi
}

La circostanza è troppo solenne.

Si tratta di salutare i coniugi Peruzzi che ne lasciano per qualche mese ${ }^{1}$.

Ma le pare! Il Fucini non può ${ }^{2}$ mancare a quest'appello.

12 Luglio 1872

\section{XXVII}

\section{R. FuCini a E. PeruZzi}

Grazie dell'invito al discorso Mamiani ma non posso dirgliene niente non essendomi stato possibile di andarvi ${ }^{1}$.

E quando rivedremo a Firenze la Sig. ${ }^{\text {a }}$ Emilia ${ }^{2}$ ? Non voglio privarla d'una notizia che la interesserà molto: Io... sono... Filocritico!

XXVI. Dall'aut. in BNCF (CEP, cass. 74, ins. 9, n. 13). Bigl. vis. adespota intestato "ING. ReNATO FuCINI". Inedito.

1. Ogni estate i Peruzzi compivano lunghi viaggi in Europa. Si veda M.P. Cuccoli, Emilia Toscanelli Peruzzi, cit., p. 201.

2. «Può» è scritto sopra una parola erasa.

XXVII. Dall'aut. in BNCF (CEP, cass. 74, ins. 9, n. 15). Bigl. vis. adespota intestato "ING. RenATO FuCINI". Data, non aut. e a lapis: Firenze 5. XII.72. Inedito.

1. Terenzio Mamiani della Rovere (Pesaro 1799-Roma 1885). Liberale, di antica famiglia nobile, trasferitosi a Firenze nel 1826, entrò in contatto con Capponi e Vieusseux, che lasciarono in lui una traccia profonda. Partecipò ai moti del 1831, in seguito al fallimento dei quali fu condannato all'esilio. Rifugiatosi a Parigi, dove diede alle stampe alcune opere filosofiche, per rientrare nel 1847 a Roma, condividendo le speranze di molti in Pio IX. Dopo il fallimento della prima guerra d'indipendenza fu nuovamente costretto all'esilio, riparò a Genova, dove fondò l'Accademia di Filosofia Italica. Eletto deputato, sostenne l'opera di Cavour, divenendo anche ministro della Pubblica Istruzione. Su di lui si vedano F. Zerella, Il pensiero sociale di Terenzio Mamiani, Roma, "Nuova rivista pedagogica", 1960; M. PICHERLE, Moderatismo politico e riforma religiosa in Terenzio Mamiani, Milano, Giuffré, 1973.

Non sappiamo con sicurezza quale fosse l'argomento della conferenza del Mamiani, svoltasi probabilmente al Circolo Filologico di Firenze (si veda V. VAnNuCCI, Istituzioni fiorentine. Raccolta di monografie dei principali Istituti di Beneficenza, Letterari, Scientifici, Educativi, Circoli di ricreazione, ecc..., cit., p. 293). Nel suo saggio biografico, Enrico Colini parla di due discorsi tenuti dal Mamiani a Firenze, il primo dei quali, riguardante «il senso morale degli italiani», nel 1868. Sul secondo, "sulla fortuna delle lingue», Colini non fornisce alcuna datazione. Cfr. E. ColinI, Notizie della vita e delle opere di Terenzio Mamiani, Jesi, Tip. Fratelli Ruggini, 1885, p. 224 e p. 251.

2. Il riferimento all'assenza della signora Emilia da Firenze fa pensare che il biglietto sia stato scritto durante l'estate, quando i Peruzzi compivano lunghi viaggi attraverso l'Europa. 
XXVIII

R. Fucini a E. PERUZZi

Gentilissima Sig. ${ }^{\text {E Emilia }}$

7 Febbraio

Pei biglietti avevo pensato alla Signora Emilia ma poi, essendomi stati offerti da un impiegato del Gabinetto, non mi è occorso incomodarla. In ogni modo la ringrazio di essersi rammentata di me.

Non mi sono fatto vedere né Lunedì, né Mercoledì, essendomi proposto, almeno per qualche tempo, il solo Sabato per complimentare i Sig. Peruzzi e la ragione si è che: tutte le altre sere, salvo rare eccezioni, mi trattengo in casa a lavorare ${ }^{1}$. Quel che scapperà fuori da questo lavoro non lo so nemmen'io, ma quanto prima spero di farlo sapere anche agli amici.

Non sapevo nulla della morte della sua Sig. ${ }^{a} \mathrm{Zia}$. Mi associo al suo dolore e incaricandola de' miei rispettosi complimenti pel Sig. Ubaldino mi onoro d'esser suo devoto servo

R. Fucini

\section{XXIX}

R. Fucini a E. PERUZZI

Sta bene per oggi alle 6 .

XXVIII. Dall'aut. in BNCF (CEP, cass. 74, ins. 9, n. 16). Lettera su 1 f. su 1 c. Anno, non aut. e a lapis: 1873. A sx della data, ann. non aut.: "F". Inedita.

1. È probabile che Fucini stesse lavorando a una nuova edizione dei Cento sonetti, pubblicata da Barbèra nel 1876 (si veda la nota 3 alla lettera LXI).

XXIX. Dall'aut. in BNCF (CEP, cass. 74, ins. 9, n. 17). Bigl. vis. adespota intestato "InG. RenATO FuCINI". Data, non aut. e a lapis: 10.3.73. Inedito 


\section{XXX}

\section{R. Fucini a E. PeruZZi}

Lunedì 17

Non è vero nulla che il Fucini sia morto, anzi è vivo e verde. Sabato andai alla Pergola ${ }^{1}$, stasera vado di guardia ai Pitti, ecco la ragione che mi fa ricorrere al calamaio per dar segno di vita. Gradisca i rispettosi complimenti del suo devotissimo servo infrascritto.

\section{XXXI}

\section{R. Fucini a E. PeruZzi}

\section{Gentil.ma Sig. ${ }^{a}$ Emilia}

La prego di un favore. Se stasera vede il Sonnino ${ }^{1}$, col quale avevo fissato di trovarmi costì per andare insieme in casa Corsini ${ }^{2}$, lo preghi a scusarmi se manco all'appuntamento, poiché vado a far nottata ad un mio amico gravemente ammalato.

La ringrazio.

XXX. Dall'aut. in BNCF (CEP, cass. 74, ins. 9, n. 18). Bigl. vis. adespota intestato "ING. RenATO FuCINI". Data, non aut. e a lapis: 3.73. Inedito.

1. Si tratta del noto teatro fiorentino, situato nell'omonima via.

XXXI. Dall'aut. in BNCF (CEP, cass. 74, ins. 9, n. 19). Bigl. vis. adespota intestato "ING. RenATO FuCINI". Data, non aut. e a lapis: 24.3.73. Inedito.

1. Giorgio Sidney Sonnino (Pisa 1847 - Roma 1924). Compì varie esperienze diplomatiche a Madrid, Vienna, Berlino e Versailles. Rientrato in Italia, si interessò alla situazione dei contadini in Toscana e in Sicilia. Insieme a Leopoldo Franchetti fondò la rivista "Rassegna Settimanale», che, trasferita nel 1878 a Roma, continuò le pubblicazioni fino al 1882, per poi diventare quotidiano indipendente con il nome «La Rassegna». Fu ministro del Tesoro nel 1894 e presidente del Consiglio per due brevi periodi, nel 1906 e nel 1909-1910. In occasione del primo conflitto mondiale, ricoprì un ruolo di primo piano nella politica nazionale: come ministro degli Esteri, sostenne la necessità che l'Italia scendesse in guerra a fianco di Francia, Inghilterra e Russia. Si veda S. Sonnino, Opera omnia, diretta da B.F. Brown, Bari, Laterza, 1972-1981, 8 voll.

2. Si tratta di una delle più antiche casate nobili di Firenze, che vantava origine feudale. I Corsini possedevano numerosi palazzi a Firenze e dintorni. Si vedano L. PASSERINI, Genealogia e storia della famiglia Corsini, Firenze, Cellini, 1858; L. GINORI LISCI, I palazzi di Firenze nella storia e nell'arte, Firenze, Cassa di Risparmio di Firenze, 1972, vol. I, pp. 147-156 e pp. 293-299; vol. II, pp. 625-628; B. CorsINI, La famiglia Corsini, Certaldo, Shakespeare and Company, 1996. 


\section{XXXII}

R. Fucini a E. PeruZzi

Il Fucini è spiacente di non poter vedere stasera la Sig. ${ }^{a}$ Emilia. La saluta e lascerà il suo piccolo obolo per i ciechi. No 5 copie de' suoi cento sonetti ${ }^{1}$.

\section{XXXIII}

R. Fucini a E. PeruZZi

Sabato e Lunedì non sono stato in città. Stasera resto dolorosamente sorpreso di sentirla partita per l'Antella e tanto più me ne dispiace per non aver potuto salutarla prima della sua partenza.

\section{XXXIV}

R. Fucini a E. PeruZZi

Gentilissima Sig. ${ }^{a}$ Emilia

\section{Lunedì 17 Febbraio}

Sta bene. La ringrazio di tutto cuore.

Suo devoto amico

R. Fucini

XXXII. Dall'aut. in BNCF (CEP, cass. 74, ins. 9, n. 20). Bigl. vis. adespota intestato "ING. RenATO FuCINI". Data, non aut. e a lapis: 7.4.73. Inedito.

1. Per i sonetti di Fucini si veda la nota 1 alla lettera XII.

XXXIII. Dall'aut. in BNCF (CEP, cass. 74, ins. 9, n. 21). Bigl. vis. adespota intestato "ING. RenATO FuCINI" scritto a lapis. Data, non aut. e a lapis: 15.5.73. Inedito.

XXXIV. Dall'aut. in BNCF (CEP, cass. 74, ins. 10, n. 1). Lettera, 1f. su 1 c. Data, non aut. e a lapis: 1873. Inedita. 


\section{XXXV}

\section{E. Peruzzi a R. Fucini}

Il mondo è davvero sottosopra! Non si fermi alle miserie ${ }^{1}$ - il Tommaseo ${ }^{2}$, il Broglio (Nazione del 26) ${ }^{3}$, il Bonghi (Perseveranza del 26) ${ }^{4}$ dicono del Manzoni parole che si leggono con piacere - il Bonghi principiò con semplicità e s'innalza poi sempre di più - è un grande ometto - bello tanto anche il discorso al Parlamento (Nazione di oggi) ${ }^{5}$. La forza dei Vangeli è stupenda.

\section{Domenica}

Per Giugno lei e il Del Corona se volete biglietti per la festa delle semole potrete averle dal Mauri - sarò anch'io quel giorno in città ${ }^{6}$. L'invito fin d'ora all'Antella per l'altra

XXXV. Dall'aut. in BRF (FRF, cass. 8, ins. 22). Bigl. vis. adespota intestato "Emilia PeruZZI". S. d. Data congetturale: 27 maggio 1873 (ricavata in base ai riferimenti agli articoli di Broglio, Tommaseo e Bonghi e alla luce della lettera XXXVI). Inedito.

1. Probabile che Fucini faccia riferimento alla vicenda riguardante un biglietto di Manzoni promessogli da Giorgini: «Allora chiesi al Giorgini di poter avere, per mezzo suo, un biglietto dell'autore dei Promessi Sposi. Al suo ritorno, sentendo che non l'avevo avuto, restò maravigliato e mi osservò: - Ma proprio, non l'hai avuto? - No! Ho avuto una semplice carta di visita, con un p.r. e basta. Capisco che per il mio merito è forse troppo, ma... - Allora è nato un malinteso. Fra pochi giorni torno a Milano, e il biglietto l'avrai. - Tornò a Milano; ma trovò gravemente ammalato il glorioso vecchio, il quale poco dopo morì» (cfr. R. FucINI, Acqua passata, cit., p. 520).

2. Si tratta probabilmente di N. TOMMASEO, Alessandro Manzoni nella diciasettesima commemorazione dall'anno che Antonio Rosmini morì, Firenze, Tipografia Cellini, 1873.

3. Emilio Broglio (Milano 1814-Roma 1892). Studiò giurisprudenza a Pavia, laureandosi nel 1835, ma per motivi politici fu costretto a rinunciare all'esercizio della professione. Nel 1848 prese parte alle Cinque giornate e fu eletto deputato al Parlamento subalpino. Dopo il ritorno degli austriaci rimase a Torino, dove si dedicò a ricerche di diritto tributario. Nel 1867 venne nominato ministro dei Lavori Pubblici, ma passò presto a sostituire il Cantelli come ministro della Pubblica Istruzione. Nel secondo governo Menabrea (1867-1868) fu ministro dell'Agricoltura e del Commercio. La Peruzzi fa riferimento all'articolo scritto da Broglio in occasione della morte di Manzoni e pubblicato sulla «Nazione» del 26 maggio 1873 con il titolo Alessandro Manzoni.

4. Ruggiero Bonghi (Napo1i 1826-Torre del Greco [Napoli] 1895). Nell'agosto del '48 si trasferì a Firenze, dove cominciò a frequentare il Gabinetto Vieusseux, stringendo amicizia, tra gli altri, con Silvio Spaventa. Allontanato dal Granducato per richiesta del governo borbonico, riparò a Torino. Fra il 1851 e il 1852 soggiornò a Parigi e a Londra, per tornare poi nella capitale del Regno di Sardegna. Passò poi a Stresa, dove ebbe l'occasione di frequentare il Manzoni, che influì soprattutto sulla sua concezione della lingua. Dal 1874 al 1876 fu ministro della Pubblica Istruzione durante il gabinetto Minghetti. Dopo la caduta della Destra, avvenimento che segnò la rottura tra il Bonghi e il salotto Peruzzi, di cui fino ad allora era stato uno dei principali animatori, fu critico severo della politica della Sinistra, soprattutto per quanto riguarda la politica estera e nel campo scolastico. Creò il «Nazionale» di Napoli e la «Perseveranza» di Milano. Su di lui si veda F. Tessitore, Crisi e trasformazione dello Stato. Ricerche sul pensiero giurisdizionale tra Otto e Novecento, Napoli, Morano, 1963, pp. 72-90; L. Bellingeri, M.G. Gajo Mazzoni, Ruggiero Bonghi fra politica e cultura, Roma, Biblioteca Nazionale Centrale, 1996. L'articolo in questione è la lettera, dal titolo Alessandro Manzoni, pubblicata sulla «Perseveranza» del 26 maggio 1873.

5. Si tratta del discorso del Bonghi al Parlamento sulla questione dei rapporti tra Stato e Chiesa, pubblicato dalla «Nazione» tra il 27 maggio e il 7 giugno 1873.

6. Su Ottaviano Del Corona si veda la nota 1 alla lettera Xx. Achille Mauri (Milano 1805-Pisa 1883). Nel 1848 stese il Manifesto del Governo provvisorio alle nazioni europee, in cui si deprecava l'atteggiamento 
Domenica 8 Giugno e il luogo del convegno alla Loggia de' Lanzi alle 4. Le ritorno la rivista [?] perché abbonata da un anno e perché già dovetti un anno fa occuparmene. Grazie dei suoi sforzi pel Fanfani ${ }^{7}$.

\section{XXXVI}

\section{R. Fucini a E. PeruZzI}

La ringrazio dell'invito ed accetto a condizione che nulla me lo impedisca. Ho letto le parole del Broglio e vi ho trovato del buono. Lo scritto del Bonghi, per ora, non ho potuto vederlo, ma lo vedrò. Di quello che ha detto lo Zannetti nella Gazzetta d'Italia di ieri che gliene pare ${ }^{1}$ ? A me sembra che vi sia dimolta ${ }^{2}$ polpa. Non posso negare che questi naturalisti hanno, da un pezzo in qua, pochi discorsi e buoni, e degli argomenti parecchio stringenti senza tante spampanate rettoriche. Io gli voglio bene ai naturalisti e ai cultori delle scienze fisiche in generale. Sono quelli che quando muoiono lasciano poco rumore di campane ma pei quali resta il vapore, il telegrafo a dichiararli i primi se non gli unici benemeriti del vero progresso.

\section{XXXVII}

\section{R. Fucini a E. PeruZzi}

Mi è assolutamente impossibile. La ringrazio tanto tanto.

tenuto dall'Austria in occasione della prima guerra d'indipendenza italiana. L'anno successivo redasse il documento con il quale il governo piemontese spiegava le ragioni della rottura dell'armistizio di Salasco. Cavour gli affidò il compito di redigere le memorie sull'Italia e sul papato da consegnare a Napoleone III. Venne nominato senatore nel 1871.

7. Pietro Fanfani (Montale [Pistoia] 1815-Firenze 1879). Il padre lo iscrisse alla scuola medico-chirurgica di Pistoia, ma, visti gli scarsi risultati, lo indusse in seguito ad arruolarsi. Nel 1842 decise di riprendere gli studi; tra il 1843 e il 1845 iniziò a collaborare con alcune riviste. Nel 1848 partì con i volontari toscani e a Montanara venne fatto prigioniero. Rilasciato, nel 1859 venne nominato bibliotecario alla Marucelliana di Firenze, posto che occupò fino alla morte, avendo rifiutato l'incarico di direttore della Biblioteca Nazionale di Napoli.

XXXVI. Dall'aut. in BNCF (CEP, cass. 74, ins. 10, n. 1 bis). Bigl. vis. intestato "ING. RenaTo FuCINI”. Data, non aut. e a lapis: 30.5.73. Inedito.

1. Arturo Zannetti (Pontedera [Pisa] 1840-Firenze 1884). Si laureò in scienze naturali all'università di Pisa. Nel 1859 combatté come volontario tra i bersaglieri toscani. Avendo vinto un concorso, nel 1862 compì studi di perfezionamento a Parigi; rientrato in Italia, ottenne la cattedra di scienze naturali a Casale Monferrato e, successivamente, a Firenze, dove per alcuni anni svolse anche la funzione di aiuto alla cattedra di antropologia occupata da Paolo Mantegazza. Non è stato possibile rintracciare l'articolo menzionato da Fucini.

2. Così nell'originale.

XXXVII. Dall'aut. in BNCF (CEP, cass. 74, ins. 10, n. 2). Bigl. vis. adespota intestato "ING. ReNATO FuCINI". Data, non aut. e a lapis: 14.VI.73. Inedito. 


\title{
XXXVIII
}

\author{
R. Fucini a E. PeruZzi
}

Io non mancherò. Del-Corona non può e mi prega di fare le sue scuse e ringraziamenti ${ }^{1}$.

\section{XXXIX}

R. Fucini a E. Peruzzi

Siccome ho in Firenze mio padre e mia madre ${ }^{1}$, spero che la Sig. ${ }^{a}$ Emilia vorrà compatirmi se rinunzio al piacere di passar la serata di Domenica all'Antella. Tanti e tanti saluti al Prezzolini al quale vorrei stringere la mano ${ }^{2}$.

Mercoledì

\section{XL}

\section{R. Fucini a E. PeruZzi}

Accetto il cortese invito della Sig. Emilia e domani mi troverò nel numero dei vendemmiatori ${ }^{1}$.

24 Sett. $73 \mathrm{R}$

Mercoledì

XXXVIII. Dall'aut. in BNCF (CEP, cass. 74, ins. 10, n. 3). Bigl. vis. adespota intestato "ING. RENATO FuCINI". Data, non aut. e a lapis: 20.6.73. Inedito.

1. Su Ottaviano Del Corona si veda la nota 1 alla lettera XX.

XXXIX. Dall'aut. in BNCF (CEP, cass. 74, ins. 10, n. 4). Bigl. vis. adespota intestato "ING. RenATO FuCINI". Data, non aut. e a lapis: 2.7.73. Inedito.

1. Sui genitori di Fucini si vedano la nota 2 alla lettera XVI e la nota 2 al biglietto Xx.

2. Luigi Prezzolini fu segretario del barone Bettino Ricasoli, sottoprefetto e, infine, prefetto. Ferdinando Martini dice di lui che fu «uno dei funzionari più intelligenti e più colti, fra quanti n'ebbe il nuovo Regno d'Italia» (cfr. F. MARTINI, Confessioni e ricordi (Firenze granducale), Firenze, Bemporad, 1922, p. 211). De Amicis lo indica come uno dei "lettori infaticabili" del salotto Peruzzi (cfr. E. De Amicis, Un salotto del secolo scorso, cit., p. 73).

XL. Dall'aut. in BNCF (CEP, cass. 74, ins. 10, n. 5). Bigl. vis. adespota intestato "Ing. Renato FuCINI". Data presumibilmente di mano di Emilia Peruzzi. Inedito.

1. Ogni anno i Peruzzi invitavano gli amici a prendere parte alla vendemmia dei loro vigneti di Montepilli, vicino all'Antella. Si veda a proposito R. FUCINI, Acqua passata, cit., pp. 515-516. 


\section{XLI}

R. Fucini a E. PERUZZi

Signora Emilia, ben trovata.

6 Novembre 1873

Accetto con tanto piacere l'invito perché mi procura il bene di rivedere tutti in un colpo i più buoni e i più cari amici ch'io mi abbia, fra i quali metto in prima fila (crepi la cortigianeria) i Signori Peruzzi e quella simpatica figura del Giacomelli ${ }^{1}$.

Il giorno a suo piacere.

Come sono stato bene in campagna! Che salute! Che tranquillità d'animo... Ah! Se sapesse come sa di sole... etc. etc. etc.

\section{XLII}

R. Fucini a E. PeruZZi

Il Del-Corona è socio ${ }^{1}$.

Mille grazie del biglietto e non mancherò di profittarne.

\section{XLIII}

R. Fucini a E. PeruZZi

Con molto dispiacere non posso accettare l'invito della Sig. ${ }^{a}$ Emilia perché sono aspettato a Dianella dai miei Genitori ${ }^{1}$. Mille grazie e i soliti rispettosi complimenti.

Li 6 Decembre 1873

XLI. Dall'aut. in BNCF (CEP, cass. 74, ins. 10, n. 6). Bigl. vis. adespota intestato "ING. Renato FucINI". Inedito.

1. Su Giacomelli si veda la nota 2 alla lettera IX.

XLII. Dall'aut. in BNCF (CEP, cass. 74, ins. 10, n. 7). Bigl. vis. adespota intestato "ING. ReNATO FuCINI". Data, non aut. e a lapis: 24 nov 73. Inedito.

1. Su Del Corona si veda la nota 1 alla lettera XXII.

XLIII. Dall'aut. in BNCF (CEP, cass. 74, ins. 10, n. 8). Bigl. vis. adespota intestato "ING. RenATO FuCINI". Inedito.

1. Sui genitori di Fucini si vedano la nota 2 alla lettera XVI e la nota 2 alla lettera XX. 


\section{XLIV \\ R. Fucini a E. PeruZZi}

La ringrazio ed accetto. Le anticipo, intanto, i miei complimenti. Lunedì.

Le invio cinque copie del barbaro epitalamio, se ne vuole dell'altre me lo dirà a voce ${ }^{1}$.

\section{XLV}

\section{R. Fucini a E. Peruzzi}

E allora non sta più bene più così. Va bene cosi?

Siccome in qualunque modo avrò il piacere di vederla stasera, il dispiacere di non assistere all' agape amichevole viene molto mitigato dal nuovo e simpatico attentato di confidenziale amicizia datomi nel suo gentile biglietto; e moltissimo poi dal pensare che probabilmente, non fo per lodarmi ma, sarei stato il più giovane della comitiva,

XLIV. Dall'aut. in BNCF (CEP, cass. 74, ins. 10, n. 9). Bigl. vis. adespota intestato "ING. RenATO FuCINI". Data, non aut. e a lapis: 19.1.74. Inedito.

1. Si tratta di un componimento scritto da Fucini in occasione delle nozze del cognato Giorgio Roster con Emma Pellizzari e pubblicato domenica 11 gennaio 1874 sul «Corriere Italiano»: «Dunque lei prende moglie! Tiri via! / Bon pro' li facci e Dio nun l'abbandoni. / Io l'imprumetto, per la parte mia, / Di rammentallo ' $n$ delle mi' orazioni; / Se poi Gesù lo vole abbandona', / Protesto che nun ci ho nulla che fa. // Ho moglie anch'io, sarà circa sett'anni, / Talché, lei vede, nun son più un monello; / Dunque, perché 'un si trovi a disinganni, / Li parlerò tar quale a un mi' fratello; / Se sbaglio me lo dica, ma, sor Giorgino, / Creda a me, son più 'nfame di Guazzino. // 'N su primi tempi [lui] vedrà unni 'osa / Risprende' tutto 'ome fussi argento; / La nebbia li parrà color di rosa; / Vedrà brilla' la terra e 'r firmamento; / A tutti vorra' da' ' $n$ pezzo di 'ore... / Ma nun si fidi: éne 'n gran gancio Amore. // Cos’è, cosa nun è, lui se la fuma / E lassa 'de' su' piedi la Realtà... / Di 'velle 'nfami! 'un c'è la peggio stiuma. / T'entra 'n casa, 'un c’è cristi, 'un se ne va'; / Sarta 'n su tetti; rampia 'n su' muri, / E tutti i 'iari l'arriduce scuri. // E allora, bimbo mio, ci vor giudizio / Tre vorte più che a fare 'n oriolo, / 'Nsennò no' si va giù 'n der precipizio: / Moglie e marito 'antano ll'assolo... / Senta, 'un se n'esce, la felicità / Consiste tutta 'n der sape' ammolla'. // Se la moglie, 'om'esse', lo seccassi / Cor dinni: - Torna 'n po'più presto a cena - / Ammolli. E se li 'iede delli spassi: / Fia' voluntas tua, pieghi la stiena... / ' $\mathrm{N}$ dello 'nverno teatri; e' $n$ dell' 'istate, / Si sa, birra, passeggi e gramolate. // Se poi cor tempo un giorno si vedessi / Sarta' 'n sulle ginocchia de' mocciosi, / Faccia ' $r$ bilancio e abbadi all'interessi / Perch' e' tempi ènno assa' 'alamitosi... / Ribasserà, ma per quest'anno 'r vino / E' l'hanno fatto ottanta franchi ar tino! // O 'r necessario! Er pane, l'olio, 'r sale... / Unni 'osa, sor Giorgino, è rincarato: / E fussi 'vello solamente 'r male: / O quelli che ci spolvera lo Stato? / Unni po' po' ti 'apita 'n Donzello... / Quanto? - Se' franchi - Tenga - Arrivedello. // In fin de' 'onti, la 'vistione è questa: / Che quando 'r brav'anello è drent'ar dito, / Bisogna mette' a rango un po'la testa / O, come si suor di', 'r capo a partito... / Er matrimonio, vede, ène 'n fagotto; / Si regge 'n dua, ma a vorte vien di sotto! // Dunque ho finito, e voglio ave' fiducia / Che trovi 'n della sua degna 'onsorte / Una donna che l'ami e li' ucia / La robba 'n casa 'nfino alla su' morte; / Una donna, dirrò come lei dice, / Che sappa sempre rendello felice. // Già 'un c'è dubbi: La sua dorce sposina / È troppo una ragazza 'om 'ir fo; / Garbata, 'nsenza muffa, belloccina... / Eppo' ha quarcosa addosso... o che lo so?... / Qualche cosa... 'om'esse'... ora 'un saprei... / Bravo! Preciso 'ome dice lei».

XLV. Dall'aut. in BNCF (CEP, cass. 74, ins. 10, n. 10). Bigl. vis. adespota intestato "ING. Renato FuCINI". Data, non aut. e a lapis: 23.1.74. Inedito. 
vale a dire la vittima innocente destinata ad espiare l'imprudenza di tutti con una morte immatura. Davvero, a che punto arriva l'istinto della propria conservazione!

\section{XLVI}

\section{R. Fucini a E. PeruZzi}

Domani sera dopo l'Uffizio vado in campagna per affari, e tornerò tardi, se non mi toccherà passare la nottata fuori di Firenze. Nel caso ch'io torni non mancherò di venire a salutarla. Intanto la ringrazio dell'invito cortese e la prego a scusarmi.

Domenica

\section{XLVII}

R. FuCini a E. PERUZZI

Sta bene così. Non le ho reso prima la risposta perché ieri sono stato tutto il giorno fuori di Firenze.

Lunedì.

\section{XLVIII}

\section{R. Fucini a E. PeruZzi}

Graditissimo quell'invito, e se ella non mi avesse prevenuto glie ne avrei fatta domanda perché sono un ammiratore dei grandi viaggiatori ${ }^{1}$. Lo Zagri ${ }^{2}$ non potrò vederlo perché stasera non vado in casa Toscanelli ${ }^{3}$ e non so dove trovarlo altrove; però se ella

XLVI. Dall'aut. in BNCF (CEP, cass. 74, ins. 10, n. 11). Bigl. vis. adespota intestato "ING. ReNATO FuCINI". Data, non aut. e a penna: 8.II.74. Inedito.

XLVII. Dall'aut. in BNCF (CEP, cass. 74, ins. 10, n. 12). Bigl. vis. adespota intestato "ING. ReNATO FuCINI". Data, non aut. e a lapis: 23.2.74. Inedito.

XLVIII. Dall'aut. in BNCF (CEP, cass. 74, ins. 10, n. 13). Bigl. vis. adespota intestato "ING. ReNATO FuCINI". Inedito.

1.Il mercoledì e il venerdì la signora Emilia invitava esperti viaggiatori perché rispondessero in modo esauriente alle domande dei suoi amici. Cfr. V. PAReto, Lettere ai Peruzzi, cit. p. 132. Si veda anche G. Rossi, Salotti letterari in Toscana, cit., p. 111.

2. Guido Zagri era un assiduo frequentatore del salotto Peruzzi.

3. Giuseppe Toscanelli (1828-1891). Partecipò ai moti e alla campagna del 1848, combattendo a Curtatone, insieme al fratello Domenico, con i volontari toscani, e in seguito prese parte anche alla difesa di Venezia. Si tratta di uno dei fratelli di Emilia Peruzzi. 
avesse qualche nomina che le restasse inoperosa, me la mandi pure domattina all'Uffizio ché io penserò a utilizzarla degnamente.

Venerdì 27 Febbraio 74

\section{XLIX}

\section{R. FuCINi a E. PERUZZI}

Sta bene così. Mille grazie anticipate. Parleremo un po' di quel gran buco nell'acqua che faranno i compilatori del nuovo vocabolario della lingua... di dove ${ }^{1}$ ?

\section{$\mathrm{L}$}

\section{R. Fucini a E. Peruzzi}

Io sono tre volte mortificato per non essermi fatto più vedere da lei da qualche giorno specialmente poi in questi momenti di grande attrito, ma mi è stato assolutamente impossibile poiché, tolto Lunedì della settimana scorsa, gli altri giorni di ricevimento sono sempre stati in vigilia di festa quando appunto io, al treno delle 4 e dieci, me ne volo a trovare la mia famiglia in campagna. Aggiunga che, per una malaugurata combinazione, vi è stato e vi è tanto da fare in uffizio che non mi è stato possibile ancora di vedere l'esposizione... eppure è così ${ }^{1} ! \mathrm{E}$ creda pure che ho un diavolo per capello. Mi perdoni molto perché me lo merito troppo.

Venerdì 15 Maggio 74

XLIX. Dall'aut. in BNCF (CEP, cass. 74, ins. 10, n. 14). Bigl. vis. adespota intestato "ING. ReNATO FuCINI". Data, non aut. e a penna:15.4.74. Inedito.

1. Si tratta probabilmente del Novo vocabolario della lingua italiana secondo l'uso di Firenze, alla cui compilazione, voluta dal ministro Broglio, stavano lavorando alcuni esponenti del salotto Peruzzi, tra cui Giorgini, Gotti, Gelli, Fanfani.

L Dall'aut. in BNCF (CEP, cass. 74, ins. 10, n. 15). Bigl. vis. adespota intestato "ING. RenATO FuCINI". Inedito.

1. Dall'11 al 25 maggio 1874 si tenne a Firenze l'Esposizione Internazionale di Orticoltura. Si veda A. BigAZZI, Firenze e contorni. Manuale bibliografico e bibliobiografico delle principali opere e scritture sulla storia, i monumenti, le arti, le istituzioni, le famiglie, gli uomini illustri, ecc., della città e contorni, Firenze, Ciardelli, 1893, p. 120. 
$\mathrm{O}$ vivo o morto, Lunedì sarò al mio posto e se il Sole si azzardasse a liquefarmi anche una goccia del tesoro affidatomi, l'avrà da fare con me.

Sabato 13 Giugno 1874

\section{LII}

R. Fucini a E. PeruZzi

Non posso per un impegno contratto prima che mi giungesse il di Lei invito. La ringrazio tanto e la prego a scusarmi.

\section{LIII}

\section{R. Fucini a E. PeruZzi}

Quantunque nulla mi secchi più che l'idea di diventar prezioso, pure anche questa volta la necessità mi costringe a renunziare al suo invito. Dopo ricevuto il suo ultimo biglietto nel quale mi accenna ad una gita all'Antella per Martedì scorso, io non pensai più a serbarmi per la Domenica e contrassi un impegno per una scampagnata alla Villa d'un mio amico su le montagne di Prato ed un altro impegno ho dovuto contrarlo per quest'altra Domenica per andare a Vicchio di Rimaggio da Rodolfo Schneider ${ }^{1}$. Nulla meno, nella settimana entrante voglio procurarmi il piacere di salutarla in persona, o solo o accompagnato, capiterò costassù insalutato ospite per contemplare dalla sua stupenda terrazza la bella cometa che campeggia nel nostro cielo fra l'Orsa maggiore e il Cupolino del Giorgi².

LI. Dall'aut. in BNCF (CEP, cass. 74, ins. 10, n. 16). Bigl. vis. adespota intestato "ING. RenATO FucINI". Inedito.

LII. Dall'aut. in BNCF (CEP, cass. 74, ins. 10, n. 17). Bigl. vis. adespota intestato "ING. RenATO FuCINI". Data, non aut. e a penna: 26.VI.74. Inedito.

LIII. Dall'aut. in BNCF (CEP, cass. 74, ins. 10, n. 18). Bigl. vis. adespota intestato "ING. RenATO FuCINI". Data, non aut. e a penna: 3.VII.74. Inedito.

1. Vicchio di Rimaggio si trova nelle vicinanze di Firenze, in Val d'Arno, nei pressi di Bagno a Ripoli.

2. Si tratta di una grande cometa scoperta a Marsiglia. Si veda «La Nazione» del 3 luglio 1874. Non è stato possibile individuare con precisione chi sia il Giorgi qui citato. Potrebbe trattarsi di Giorgio Giorgi (Firenze 1836-Roma 1915), senatore e presidente del Consiglio di Stato. 


\section{LIV}

R. Fucini a E. PeruZzi

\section{Li 25 Luglio 1874}

Eccole la pianta richiestaci a Del-Corona ed a me ${ }^{1}$. Si spera che tutto corrisponda ai di Lei desiderj e facendo i più caldi augurj tanto a Lei che al Sig. Ubaldino per un viaggio de' più fortunati, ci onoriamo segnarci di lei devot.mi amici

R. Fucini

Ottaviano Del-Corona

\section{R. Fucini a E. PeruZzi}

Che peccato ch'io non possa profittare di questa bella occasione poiché mi sento male! Mi sentivo già non troppo bene domenica scorsa costassù all'Antella e il giorno dopo stetti a letto con febbre e dolore di gola e ancora non ho ripreso la mia piena vitalità, causa forse questo caldo eccessivo. Ho letto in un fiato la Miranda ${ }^{1}$. Se presto potrò venire a trovarla glie ne dirò a voce le mie impressioni, se nò le scriverò.

Grazie tante del gentilissimo suo pensiero e di una preferenza che tanto lusinga il mio amor proprio.

LIV. Dall'aut. in BNCF (CEP, cass. 74, ins. 10, n. 19). Bigl. vis. intestato "Ing. Renato FuCinı". Entrambe le firme aut. Inedito

1. Su Del Corona si veda la nota 1 alla lettera XXII.

LV. Dall aut. in BNCF (CEP, cass. 74, ins. 10, n. 20). Bigl. vis. adespota intestato "ING. RenATO FuCINI". Data, non aut. e a lapis: 21.7.74. Inedito.

1. Poemetto polimetrico di Antonio Fogazzaro (Firenze, Le Monnier, 1874), incontrò successo di critica e pubblico. Consta di un proemio narrativo, La Lettera, di due diari, Il libro d'Enrico e Il libro di Miranda, e di un epilogo pure narrativo, Da te, da te, solo da te. Emilia richiese, oltre a quella di Fucini l'opinione di molti altri frequentatori del suo salotto e tenne informato Fogazzaro circa i giudizi espressi da essi (cfr. L.M. Fortunato De Lisle, The circle of Pear, cit., p.550). Si veda anche A. Toscanelli Altovita Avila, Emilia Toscanelli Peruzzi. Notizie e ricordi, cit., p. 33: «La zia titubava nel suo consenso di ammirazione, quando Fogazzaro le inviava Miranda. Essa faceva leggere a tutti il piccolo poema, e domandava giudizi $[\ldots] »$. 


\section{R. Fucini a E. PeruZzi}

Ella è impagabile. Grazie tante e di grandissimo cuore anche per parte del Pacchi che imparerà ad ammirare quella Provvidenza incarnata che si chiama "La Sig. a Emilia Peruzzi”. Domattina verrò a salutarla e ad augurarle tutto quel bene che Ella si merita.

\section{Li 31 Decembre 1874}

\section{LVII}

\section{R. Fucini a E. PeruZzi}

La Principessa Obbrenovich m'ha empito la casa di grazia di Dio $e^{1}$, fra le altre cose mi ha consegnato una busta contenente una lettera per Lei e una somma in fogli di banca.

La stessa Sig. Principessa ha mostrato desiderio che ella abbia presto tutta questa roba, ma come si fa? Che devo mandargliela all'Antella? Che devo lasciargliela al palazzo in Firenze? Mi dica qualche cosa con sollecitudine, perché vorrei che questa generosa Signora avesse presto una risposta. Io le avrei mandato la busta coi danari, ma siccome io ne sono responsabile, mi piace poco di farla sballonzolare per le vie maestre e vorrei consegnargliela in proprie mani.

Li 5 del 1875

\section{LVIII}

\section{R. Fucini a E. PeruZzi}

\section{Li 71875}

La principessa Obrënovich (ho potuto saperlo da chi l'avvicina) è dispiacentissima di non aver ricevuto sue lettere. Pensando a quanto ella fu premurosa nel chiedermi

LVI. Dall' aut. in BNCF (CEP, cass. 74, ins. 10, n. 21). Bigl. vis. adespota intestato "ING. Renato FucINI". Inedito.

LVII. Dall' aut. in BNCF (CEP, cass. 74, ins. 11, n. 1). Bigl. vis. adespota intestato "ING. Renato FucINI". Inedito.

1. Si tratta di Maria Obrenovich, madre del principe di Serbia Milan IV, giunta a Firenze il 22 novembre 1874. Cfr. «La Nazione» di lunedì 23 novembre 1874.

LVIII. Dall'aut. in BNCF (CEP, cass. 74, ins. 11, n. 2). Bigl. vis. adespota intestato "ING. ReNATO FuCINI". Data, non aut. e a lapis: Gen. Inedito. 
l'indirizzo di questa signora, mi nasce il dubbio che un suo biglietto possa essere andato smarrito o che debba averlo ricevuto subito dopo esternato il suo dispiacere. Stasera saprò qualche cosa alla locanda, ma intanto ho voluto scriverle per informarla.

\section{LIX}

\section{R. Fucini a E. PeruZZi}

\section{Mercoledì}

Domani alle 4 precise sarò alla Loggia de' Lanzi. Il Roster credo che non potrà venire (suppongo che ci sia stato un invito anche per lui nella lettera che gli ho fatto recapitare) perché da due giorni è in Campagna con la sua famiglia ${ }^{1}$.

\section{LX}

\section{R. Fucini a E. PeruZZi}

Il Roster positivamente non potrà venire. Ho saputo stamani da suo padre che da due giorni è in Villa Pellizzari dove si è ammalato ed è tuttora in letto ${ }^{1}$. Povero Giorgio! Tanti complimenti dal suo devoto

R. Fucini

\section{LXI}

\section{R. Fucini a E. PeruzZi}

Gentilissima Sig. ${ }^{a}$ Emilia

7 Luglio 1875

Mille volte grazie. Cinque anni mi sembrano un po' troppi, ma qualora le offerte stassero in rapporto col lungo tempo domandato, sarei disposto anche a questo ${ }^{1}$;

LIX. Dall' aut. in BNCF (CEP, cass. 74, ins. 11, n. 3). Bigl. vis. adespota intestato "ING. Renato FuCINI". Data, non aut. e a lapis: 19.5.75. Inedito.

1. Su Giorgio Roster cfr. la nota 2 alla lettera XIV.

LX. Dall'aut. in BNCF (CEP, cass. 74, ins. 11, n. 4). Bigl. vis. intestato "ING. Renato FuCinI". Data, non aut. e a lapis: 23.V.75. Inedito.

1. La famiglia Pellizzari possedeva una villa a Montepiano, nel comune di Vernio, a nord di Prato.

LXI. Dall'aut. in BNCF (CEP, cass. 74, ins. 11, n. 5). Lettera, 3 ff. su 2 cc. Inedita.

1. Così nell'originale. 
Per dire il vero preferirei cedere l'edizione per quel numero di copie che piacesse al Sig. Barbèra ${ }^{2}$ senza altri obblighi ${ }^{3}$ né da una parte né dall'altra, ma su questo rifletterò e, se verremo a trattative, spero ci intenderemo benissimo.

Quello che io posso dare di inedito occuperà circa quaranta pagine di un serto come quello adottato nella prima stampa dei sonetti, non contando il nuovo indice, il frontespizio etc. etc ${ }^{4}$.

La persona alla quale avrei posto gli occhi addosso per farla agire da mio incaricato, sarebbe Guido Vimercati ${ }^{5}$. Che glie ne pare? Io lo crederei molto adatto sotto tutti i rapporti, ma se poi Ella avesse da propormi qualche altro nostro comune amico, me lo dica.

L'invito per Domenica non posso accettarlo, e me ne dispiace, perché vengono a Firenze i miei Genitori a passar la giornata con noi ${ }^{6}$.

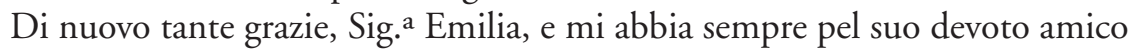

R. Fucini

P.S: Le corazzate per vendicare gli insulti scagliati contro il mio pacifico Berretto da notte non sono ancora in perfetto piede di guerra ${ }^{7}$, ma quanto prima lo saranno.

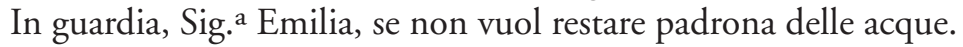

2. Prospero Barbèra (Torino 1818-Firenze 1880). Iniziò a lavorare presso la libreria Fiore e poi, nel 1840, presso l'editore Fumagalli di Firenze. L'anno successivo venne assunto da Le Monnier, per cui lavorò per quattordici anni, fino a quando, nel 1854, decise di aprire, sempre a Firenze, una stamperia per proprio conto in società con i fratelli Beniamino e Celestino Bianchi. Divenuto unico proprietario nel 1859, acquistò in breve tempo fama come editore di autori contemporanei, stampando, fra le altre, le opere di Tommaseo, Gioberti, Capponi, D’Azeglio, Mamiani. Importante fu la collaborazione con Carducci, che curò le edizioni di Cino da Pistoia, Poliziano, Alfieri, Monti e Tassoni. Dal 1859 al 1870 stampò il giornale «La Nazione».

3. Cassato «d».

4. Si tratta di R. Fucini, Cento sonetti in vernacolo pisano. Nuove poesie, Firenze, Barbèra, 1876. Si veda Annali bibliografici e catalogo ragionato delle edizioni di Barbèra, Bianchi e comp. e di G. Barbèra con elenco di libri, opuscoli e periodici stampati per commissione. 1854-1880, Firenze, Barbèra, 1904, p. 431.

5. Guido Vimercati (Venezia 1847 - ?). Compì gli studi a Torino, dove la famiglia era emigrata, nell'Istituto Tecnico, nell'università e nella scuola d'applicazione degli ingegneri. Stabilitosi nel 1869 a Firenze, pubblicò numerosi articoli di scienza e tecnologia su varie riviste e fondò la «Rivista ScientificoIndustriale». Fu professore di matematica alla scuola tecnica "Leon Battista Alberti" e di tecnologia alla Scuola commerciale di Firenze. Dal 1889 al 1895 fu tra i consiglieri della Società delle ferriere italiana, nata nel 1880 per iniziativa, tra gli altri, di Ubaldino Peruzzi.

6. Sui genitori di Fucini si vedano la nota 2 alla lettera XVI e la nota 2 alla lettera XX.

7. Non sappiamo a cosa Fucini alluda in questo post scriptum. 


\section{LXII}

\section{E. PeruZZi a R. FuCini}

Lei è un indegno e lo punisco il suo sonetto sulla pena di morte ${ }^{1}$ le ha valso un opuscolo dal bravo Paoli Consigliere della Corte di Cassazione abolizionista di grande valore - e un altro del Conte De Gori ${ }^{2}$. Li avevo qui per darglieli e lei non è venuto né Mercoledì né Sabato - a rivederla domani sera al Circolo per le parole 3 .

\section{LXIII}

\section{R. Fucini a E. PeruzZi}

\section{Luglio 1875}

Ho ricevuto il suo bigliettino un poco in ritardo perché sono stato 3 giorni a letto con febbre reumatica e i bravi custodi dell'Uffizio crederon bene d'aspettare la mia guarigione senza farmelo recapitare a casa. Nuovamente la ringrazio di tutto. Ho già veduto il Sig. Gelli e con lui ho già organizzato i preliminari del nostro affare ${ }^{1}$. Dunque alla parte?

LXII. Dall'aut. in BRF (FRF, cass.8, ins. 22). Bigl. vis. adespota intestato "Emilia PeruzzI". Scritto nel 1875, come si ricava dal riferimento al sonetto di Fucini (si veda la nota successiva). Inedito.

1. È il sonetto Al senatore $N$. N. (dopo il voto sulla pena di morte del dì... 1875): «Illustre e venerabile Signore, / Io sottoscritto Macellaro smesso, / Di lei servo devoto e ammiratore, / Umilmente Le espongo quanto appresso: // Sanguinario di nascita e di core, / Per più omicidi già sotto processo, / Vorrei, senza rischiar pelle e onore, / Servire il mio paese e far lo stesso. // Perciò, potendo aver, grazie al Senato, / Il posto di carnefice Toscano, / Gliene sarei personalmente grato. // Eccellenza, ho famiglia... Ella è sì umano / Da non sprezzare l'artista onorato, / Che chiede oprar col senno e colla mano. // Scusi tanto l'incomodo e la noia. // Mansueto Tranquilli detto Il Boia» (cfr. R. FucINI, Guazzabuglio, cit., pp. 998-999).

2. Baldassarre Paoli (Firenze 1811-1889). Si laureò in legge a Pisa ed entrò nella magistratura, diventando primo presidente della Corte d'Appello. Fu consigliere comunale e provinciale della sua città, accademico dei Lincei e amministratore di istituti di beneficenza. Sostenne l'abolizione della pena di morte. Probabilmente, l'opuscolo cui fa riferimento la Peruzzi è Osservazioni preliminari sul progetto del nuovo codice penale presentato dal Ministro Vigliani al Senato del Regno nella tornata del 24 febbraio 1874, Genova, Regio Istituto dei Sordo-muti, 1875. Augusto De Gori Pannilini (Siena 1820-1877). Di famiglia benestante, compì buoni studi, interessandosi soprattutto all'economia politica. Nel 1848 fu convinto assertore della necessità che gli stati italiani si unissero in una confederazione, ma nel decennio successivo si volse alla politica piemontese unitaria. Dopo l'annessione della Toscana al Regno di Sardegna, venne nominato senatore (1860). Prese parte attivamente ai lavori del Senato e rappresentò il governo italiano in varie esposizioni europee. Non è stato possibile individuare quale sia l'opuscolo di cui Emilia parla.

3. Si veda lettera v, nota 5. Sul Circolo Filologico cfr. la nota 2 alla lettera v.

LXIII. Dall'aut. in BNCF (cass. 74, ins. 11, n. 6). Bigl. vis. adespota intestato "Ing. Renato FuCInI". Inedito.

1. Agenore Gelli (Firenze 1829-1887). Segretario della deputazione di Storia Patria, fu direttore dell' "Archivio Storico Italiano" dal 1867 fino alla morte. Insegnò storia presso il liceo "Dante" di Firenze, dove ebbe fra i suoi allievi il Sonnino. Forse Fucini, probabilmente su consiglio della Peruzzi, aveva scelto il Gelli, invece di Vimercati, come "mediatore" con l'editore Barbèra in occasione della riedizione dei Cento sonetti in vernacolo pisano. Si veda la lettera LXI. 
E ci rivedremo, quando? forse a Novembre, mi figuro. Come vogliono essere lunghi questi mesi, specialmente per chi rimane! Dispiacentissimo di non averlo potuto fare a voce, auguro a Lei ed al Sig. Ubaldino il più brillante e il più fortunato de' viaggi.

\section{LXIV}

\section{R. Fucini a E. Peruzzi}

Salute ai reduci. Ella starà benissimo, e me ne rallegro. Una passeggiata da Firenze a Londra non può stancare chi affrontò impunemente le ultime ghiacciaje della Scandinavia ${ }^{1}$. Grazie alle di lei premure, mi sono già inteso col Barbèra ed in modo, credo, per me assai vantaggioso. Verrei a salutarla ma per ora mi è impossibile con le tante seccature che mi molestano, fra le quali è per me già colossale quella d'uno sgombero precoce. Il Roster sarà qui ai primi di Settembre ${ }^{2}$. Dio voglia che non siano gettati al vento i grandi sacrifizi che questo caro giovane ha dovuto sopportare per la sua salute.

Mi rammenti al Sig. Ubaldino e creda alla rispettosa ma cordiale amicizia del suo devotissimo

\section{R. Fucini}

Li 20 Agosto 1875

\section{LXV}

\section{R. Fucini a E. PeruZzI}

Il mio nuovo quartiere è posto in Piazza D'Azzeglio ${ }^{1}$ No $^{\circ} 143^{\circ}$ P.no ma non andrò ad abitarlo fino al 3 o 4 del mese prossimo. Per Domenica non accetto l'invito perché andrò in campagna a trovare la famiglia. Per quest'altra Domenica 5 mi tenga impegnato. Del Roster nessuna nuova oltre il dispaccio Lavarello da S. Vincenzo dove si dice - la salute a bordo è ottima $-^{2}$.

Per le feste sarò a Firenze... diavolo mai! Grazie del pensiero per mia moglie ${ }^{3}$. Col mio cannocchiale, dalla terrazza del nuovo quartiere vedo benissimo l'Antella tanto da distinguere

LXIV. Dall'aut. in BNCF (CEP, cass. 74, ins. 11, n. 7). Bigl. vis. intestato "Ing. Renato Fucinı". Data, non aut. e a penna: 20.8.75. Inedito.

1. Fucini fa qui riferimento all'annuale viaggio compiuto da Emilia e Ubaldino Peruzzi.

2. Su Giorgio Roster si veda la nota 2 alla lettera XIV.

LXV. Dall'aut. in BNCF (CEP, cass. 74, ins. 1, n. 8). Bigl. vis. adespota intestato "ING. Renato FucINI". Inedito.

1. Così nell'originale.

2. San Vincenzo si trova sulla costa toscana. Su Roster cfr. la nota 2 alla lettera XIV.

3. Su Emma Fucini si veda la nota 1 alla lettera II. 
se c'è gente alle finestre della torre. E perché non si potrebbe stabilire un telegrafo a segnali? Chi sa che questa idea non abbia in seguito uno sviluppo e possa renderci dei servigi!

Dunque a Domenica 5.

Li $26^{4}$ Agosto 1875 .

\title{
LXVI
}

\author{
R. Fucini a E. PeruZZi
}

29 Settembre 1875

Questa settimana è quella che precede la mia partenza per la villeggiatura. Per godermi in santa pace questi po' di giorni di campagna, ho tante cose da sistemare che, in verità, il lasciare Firenze ora anche per mezza giornata mi scomoderebbe troppo. Si figuri se avrebbe invitato la lepre a correre! ma ormai fino a Novembre io non son più io e per un mese brucio le navi.

Se le potesse occorrere qualche cosa da me, l'indirizzo è questo - Empoli per Vinci -. Intanto la saluto con affetto e mi congedo da lei, dal Sig. Ubaldino e dagli amici ai quali mi rammenterà come un vendemmiatore mancato ${ }^{1}$.

\section{LXVII}

\section{R. Fucini a E. PeruZZi}

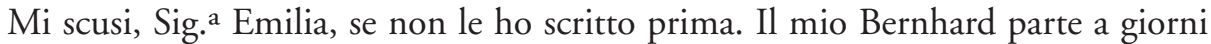
per la Germania ed io, per sistemare quà i suoi affari prima della sua partenza, da otto giorni sono talmente sottosopra, fra peripezie, disegni, contratti, sessioni, et et..., che ho dimenticato perfino gli amici ${ }^{1}$. Del libro del Giacomelli mene sono occupato e séguito ad occuparmene ${ }^{2}$. La saluto distintamente.

Venerdì 19 Novembre 1875

\section{Cassato «5».}

LXVI. Dall'aut. in BNCF (CEP, cass. 74, ins. 11, n.9). Bigl. vis. adespota intestato "ING. ReNATO FuCINI". Inedito.

1. Sull'annuale vendemmia a Montepilli si veda la nota 1 alla lettera XL.

LXVII. Dall'aut. in BNCF (CEP, cass. 74, ins. 11, n. 10). Bigl. vis. adespota intestato "ING. RENATO FuCINI". Inedito.

1. A Fucini, che aveva perduto il lavoro di aiuto-ingegnere in seguito del trasferimento della capitale a Roma, fu commissionata dal tedesco Bernhard la costruzione di un villino in via dei della Robbia (cfr. A. Zanella, Renato Fucini: studio biografico-critico, cit. p. 17).

2. Nel testamento Giovan Battista Giacomelli (su di lui si veda la nota 2 alla lettera Ix) affidava alla Peruzzi un volume contenente i suoi componimenti, perché ella si occupasse della pubblicazione (cfr. M. 


\title{
LXVIII
}

\author{
R. Fucini a E. PeruZzi
}

10 Decembre 1875

Chi non muore si rivede... oooh! Son vivo, verde, sano e contentissimo per essermi levato d'attorno una Odissea di seccature e fra qualchè ${ }^{1}$ giorno mi vedrà capitare all'Antella a salutare la più eccellente signora ch'io m'abbia mai conosciuto ed a ringraziare in persona il Sig. Ubaldino per i suoi buoni ufficj in mio favore presso il Conte Digny ${ }^{2}$.

Ieri sera tardi vidi Bistino e lo trovai assai bene ${ }^{3}$. I medici me ne hanno parlato in modo molto rassicurante. Dio non voglia che non s'ingannino.

Ma che freddo! Stanotte nevicherà.

E il suo ritorno a Firenze quando ${ }^{4}$ ? Anche il Roster sta bene ${ }^{5}$.

\section{LXIX}

\section{R. Fucini a E. PeruZzi}

Ella mi perdonerà se non corrisposi al suo gentile invito dell'altra sera, quando le avrò detto che sono in mezzo ai malati di famiglia.

Tabarrini, G. B. Giacomelli, in G. B. Giacomelli, Poesie con un discorso di Marco Tabarrini, Firenze, Le Monnier, 1876, p. 36). La Peruzzi si sarà forse rivolta al Fucini affinché l'aiutasse a trovare un editore per questa raccolta di versi.

LXVIII. Dall'aut. in BNCF (CEP, cass. 74, ins. 11, n. 11). Bigl. vis. adespota intestato "ING. RENATO FucINI". Inedito.

1. Così nell'originale.

2. Luigi Guglielmo Cambray-Digny (Firenze 1820- Firenze 1906). Avviato dal padre agli studi tecnici, fu condotto nel 1835 a Parigi per studiare. Tornato in Toscana, fu priore del Municipio sotto il gonfaloniere Ubaldino Peruzzi. Conservatore ed economista, venne nominato senatore nel 1860, dedicandosi all'amministrazione della finanza pubblica sia come membro della commissione permanente di finanza che come Ministro delle finanze (1867-1869). Dopo quest' incarico si dedicò all'unificazione del catasto fondiario nazionale. Nel 1874 fu tra i fondatori della Società Adamo Smith.

3. Si tratta di Giovan Battista Giorgini, chiamato dagli amici Bista o Bistino (cfr. G. Spadolini, Firenze capitale. Gli anni di Ricasoli, Firenze, Cassa di Risparmio di Firenze, 1979, p. 29). (Lucca 1818-Montignoso [Massa] 1908). Laureatosi in giurisprudenza a Pisa nel 1838, fu nominato professore di istituzioni criminali presso l'università di Siena; ottenne poi la cattedra di istituzioni canoniche a Pisa, dove conobbe Vittoria, figlia di Alessandro Manzoni, con la quale si sposò. Nel 1848 si aggregò al battaglione di universitari che partì dalla città toscana alla volta dell'Italia settentrionale per combattere l'Austria. Dalla fine degli anni Sessanta iniziò ad assumere un atteggiamento sempre più defilato dalla politica e riscoprì l'interesse per lo studio letterario e linguistico-lessicografico. Partecipò all'ideazione e alla redazione del Novo vocabolario della lingua italiana (1870-1897), voluto dal ministro della Pubblica Istruzione Broglio.

4. Durante i mesi più caldi dell'anno i Peruzzi si trasferivano nella loro villa dell'Antella. Cfr. M. P. Cuccoli, Emilia Toscanelli Peruzzi, cit., p. 201.

5. Su Roster cfr. la nota 2 alla lettera XIV.

LXIX. Dall'aut. in BNCF (CEP, cass. 74, ins. 11, n. 12). Bigl. vis. adespota intestato "ING. ReNATO FuCINI". Inedito. 
La moglie del dott. Roster è in letto con una febbre tifoidea e può figurarsi se l'allarme è generale e terribile ${ }^{1}$. Mia moglie poi fino dalla vigilia di Ceppo è in letto con febbre ma per ora ${ }^{2}$, grazie a Dio, senza nessun fenomeno allarmante. I medici sono tranquillissimi nel conto di lei, ma io non lo sono troppo.

Tanti buoni augurj a lei e al Sig. Ubaldino.

27 Decembre 75

\section{R. Fucini a E. Peruzzi}

Sono stato tre giorni fuori di Firenze e però rispondo con ritardo al suo gradito biglietto.

Mi giunge sempre carissimo un pensiero di lei. Presto ci rivedremo: Sabato forse se il vedere amici in questo momento non la disturba.

\section{LXXI}

\section{R. Fucini a E. PeruzZi}

5 Aprile 1876

Il Comm. Gotti ha scritto oggi al Ministro Coppino relativamente al mio impiego nelle Gallerie ${ }^{1}$. Abbia la gentilezza di avvertire il Sig. Ubaldino perché faccia a mio

1. Si tratta di Emma Pellizzari, moglie di Giorgio Roster.

2. Su Emma Fucini cfr. la lettera II, nota 1. In Toscana la festa di Natale era chiamata "Ceppo" per l'usanza di ardere il più stagionato e grosso ciocco di quercia o ulivo la sera della vigilia di Natale. La parola "ceppo" aveva anche il significato di "regalo", probabilmente per l'abitudine di regalare un ciocco di legno stagionato a chi ne era privo o come augurio. Cfr. L. ARTUSI, S. Gabbrielli, Feste e giochi a Firenze, Firenze, Becocci, 1976, pp. 85-86.

LXX. Dall'aut. in BNCF (CEP, cass. 74, ins. 11, n. 13). Bigl. vis. adespota intestato "ING. RenATO FuCINI". Data, non aut. e a lapis: 30.3.76. Inedito.

LXXI. Dall'aut. in BNCF (CEP, cass. 74, ins. 11, n. 14). Bigl. vis. adespota intestato "ING. ReNATO FuCINI". Inedito.

1.Aurelio Gotti (Firenze 1833-Roma 1904). Di modeste origini, si iscrisse contemporaneamente alla Facoltà di legge e di lettere di Pisa. A questi anni di studio risalgono i primi approcci con esponenti di spicco della cultura del tempo, come Capponi, Tabarrini e Lambruschini. Nel 1864 divenne direttore delle Regie Gallerie, impegnandosi in due obiettivi principali: l'incremento delle collezioni e la ricerca di sedi più dignitose per le raccolte artistiche fiorentine. Sospeso dalla carica di direttore, il Gotti venne giudicato colpevole di peculato continuato e di violazione dolosa dei propri dover d'ufficio, ma fu assolto in appello. Dal 1887 si dedicò, insieme al Tabarrini, alla pubblicazione del monumentale epistolario di Bettino Ricasoli (Lettere e documenti del barone Bettino Ricasoli) e iniziò a collaborare alla stesura del Novo vocabolario della lingua italiana secondo l'uso di Firenze.

Michele Coppino (Alba [Cuneo] 1822-1901).Conseguita la laurea in belle lettere a Torino nel 1844, si dedicò all'insegnamento della retorica, componendo nel frattempo alcune liriche di argomento religioso e patriottico. Nel 1867 fu nominato da Rattazzi ministro della Pubblica Istruzione, carica che ricoprì 
riguardo quello che crederà migliore e intanto ardirei pregare anche la infaticabile e benefica sua penna a voler scricchiolare sopra un pezzetto di carta, aiutandomi così a rampicare sul calvario del pane quotidiano.

Le domando scusa di tante seccature e mi ripeto come sempre suo devotissimo e rispettoso amico

R. Fucini

\section{LXXII}

\section{R. Fucini a E. PeruZzi}

Sabato 17 Giugno 1876

È molto tempo, forse troppo tempo, che ella non vede mie lettere. Non mi dia di trascurato perché questo titolo ammetterebbe un periodo antecedente di assiduità. Mi dia di Fucini ed avrà detto tutto quello che mi merito. Due mesi di ritiro campestre mi hanno fatto molto bene al fisico, punto al morale. Giovedì scorso non potei ma quest'altro verrò immancabilmente a salutarla. Ho visto la Sig. ${ }^{a}$ Vittorina ${ }^{1}$. È molto afflitta e la ragione vi è e troppo grande; però trovai l'aspetto di Bistino assai soddisfacente $^{2}$. Le chiedo un favore. Fra le sue carte ella deve avere certamente molti autografi di notabilità politiche, artistiche, scientifiche, letterarie et etc. Avrebbe da cedermi qualche scarto per una collezione?

$$
\begin{array}{r}
\text { La saluta il suo devotissimo } \\
\text { R. Fucini }
\end{array}
$$

\section{LXXIII}

\section{R. Fucini a E. PeruZzi}

Trovandomi di passaggio a Firenze, ho naturalmente domandato notizie della Sig. Emilia agli amici comuni, ed avendo inteso come ella sia dietro a fare i preparativi pel suo viaggio, ho dovuti rinunziare al piacere di fare una corsa all'Antella per salutarla. Accetti invece i miei più sinceri augurj e ci rivedremo quando tutte le pecorelle smarrite

anche nel 1876, occasione in cui preparò un progetto di riforma. Fu ancora al ministero della Pubblica Istruzione fra il 1878 e il 1879. Sulla sua attività di ministro si veda E. CATARSI, Il ministro Michele Coppino e l'istruzione elementare, in «Studi di Storia dell'educazione», I (1980), pp. 28-48; D. BERTONI JOVINE, La scuola italiana dal 1870 ai giorni nostri, Roma, Editori riuniti, 1958, passim.

LXXII. Dall'aut. in BNCF (CEP, ins. 11, n. 15). Bigl. vis. intestato "ING. RENATO FuCINI". Inedito.

1. Vittoria Manzoni, figlia del celebre scrittore e moglie di Giorgini.

2. Su Giovan Battista Giorgini cfr. lettera LXVIII, nota 3.

LXXIII. Dall'aut. In BNCF (CEP, cass. 74, ins. 11, n. 16). B. vis. Inedito. 
ritornano all'ovile, vale a dire nel Novembre. S'intenda che gli augurj sono divisibili a parti uguali fra lei e il Sig. Ubaldino al quale farà i rispettosi complimenti del suo devoto amico

20 Luglio $1876^{1}$

R. Fucini

\section{LXXIV}

E. Peruzzi a R. Fucini

10 Ottobre 1876

Ottobre.

La sua lettera, saluto pel ritorno, non mi giunse mai. Di quei versi ignorammo persino l'esistenza e l'avessimo pur saputa, l'idea di affibbiare a lei una cosa che potesse dolerci non mi sarebbe neppur passata per la mente. Dell'Oriente, de' turchi, dei serbi e dei russi, novelli crociati, ne parleremo, ché lo scriverne sarebbe cosa lunga e scolorita. Le mando per la posta una copia del libro del Giacomelli ${ }^{1}$; l'ultimo sonetto è bello e commovente; e le pagine del Tabarrini un vero gioiello di cui sono certa ella pure sarà contentissimo ${ }^{2}$. Bisogna proprio essere un beato Ermolao per non dire una parola di politica e di elezioni, in mezzo a tanto fervore di discorsi, di banchetti e intrighi. Mi scriva un po' che cosa segue costà a Empoli; raccontano che le istituzioni di beneficenza del paese siano quelle che più abbiano a lodarsi della lotta, ricevendo doni tanto dal M.se Incontri quanto dal Sig. Gargini ${ }^{3}$. Quali di questi due candidati ha secondo il giudizio suo probabilità di riuscire? Sono tutti e due deputati di destra? I progressisti non hanno

1. La data è scritta obliquamente per mancanza di spazio.

LXXIV. Dall'aut. in BRF (FRF, cass. 8, ins. 19). Lettera intestata "EP" e listata a lutto, 4 ff. su 2 cc. Indirizzo sulla busta: «Ill.mo Signore / Sig. re Cav. Renato Fucini / Empoli / per Vinci». Timbro post. di partenza: 10-10-76. Inedita.

1. Si tratta di G. B. Giacomelli, Poesie con un discorso di Marco Tabarrini, cit. Su di lui si veda la nota 2 alla lettera IX.

2. Marco Tabarrini (Pomarance [Pisa] 1818-Roma 1898). Studiò legge all'università di Pisa, conseguendo la laurea nel 1842 e ottenendo l'abilitazione a esercitare la professione forense nel 1846. Nel 1848 si arruolò volontario col grado di capitano e collaborò a varie riviste patriottiche quali «Il Contemporaneo», «Il Conciliatore», di cui fu anche direttore, e «L'Italia». Fu tra i fondatori della rivista "Archivio Storico Italiano», curandone la pubblicazione dopo la morte del Vieusseux. Alla caduta della dinastia lorenese nel 1859 fiancheggiò l'opera politica di Bettino Ricasoli, ricoprendo il dicastero della Pubblica Istruzione. Nel 1871 vene nominato senatore ed eletto segretario del Senato. Socio dell'Accademia della Crusca, svolse anche il ruolo di segretario e di arciconsolo. Dettò alcune biografie di contemporanei, tra le quali quella di Ubaldino Peruzzi, e curò, insieme al Gotti, la pubblicazione delle lettere del barone Ricasoli.

3. Ludovico Carlo Incontri (Firenze 1836-1880). Prese parte attiva agli avvenimenti politici del 1859 e, in seguito, si dedicò alla carriera diplomatica, che abbandonò quando era segretario di legazione a Pietroburgo. Ricoprì cariche amministrative nel comune e nella provincia di Firenze. Sulla «Nazione» del 17 ottobre 1876 è presente un articolo in cui si fa riferimento alla gara di elargizioni benefiche intrapresa dal marchese Incontri e da Omero Gargini in vista delle elezioni politiche del 6 novembre 1876 , vinte dall'Incontri. 
costà nessun pretendente? E lei per chi si dichiara? E quali sono le ragioni che muovono la sua preferenza? Si rammenti di adempiere scrupolosamente e attivamente, mettendo in assoluto riposo Sant'Ermolao, il suo dovere di cittadino elettore, e se dovesse questo condurla a Firenze, le porte dell'Antella, e quasi quasi le braccia dei padroni di casa, le sono aperte. Le nuove di Bistino sono ora consolanti, almeno così mi scrive Vittoria ${ }^{4}$. Dico poi a Lei partecipando alla gioia che deve provare insieme alla sua cara signora, che la Vittoria mi da ottime nuove del bravo Roster, e deplora vivissimamente di perderne i consigli e la compagnia 5 . Se Ella non viene per il 4, (ne sarà rimessa la celebrazione al dì 5 , giorno della elezione) è però in stretto dovere d'invocare le muse e di mandare un sonetto o qualsivoglia poesia da leggersi sul finire delle mense ${ }^{6}$. La saluto a nome degli amici; spero che la solitudine e l'osservazione attenta della natura e degli uomini le ispiri quelle poesie che fanno poi a tutti tanto piacere. Lesse nella Nazione le belle lettere del Barazzuoli in risposta al Tommasi Crudeli ${ }^{7}$ ? Il Sindaco di Firenze fu difeso dai fatti. Le cerchi e le legga. Da sua Emilia.

\section{LXXV \\ E. PeruzZi a R. Fucini \\ Poesie Giacomelli ${ }^{1}$}

Non basta ritornare dal Bosforo, non basta esser sfuggita di mano a Turchi e Serbi perché Ella prenda in mano la penna e mi mandi un suo rigo ${ }^{2}$ ? Che occorre adunque per

4. Su Giorgini si veda la lettera LXVIII, nota 3. Vittoria sarà probabilmente Vittoria Altoviti Avila (Firenze 1827-1896), moglie di Giuseppe Toscanelli, e dunque cognata di Emilia. Di grande cultura, fece della sua casa un centro di studi, manifestando un particolare interesse per la zoologia.

5. Su Giorgio Roster cfr. la nota 2 alla lettera XIV.

6. Il 4 di Novembre di ogni anno i Peruzzi davano un pranzo per festeggiare i quattro Carli loro amici: Carlo Hillebrand, Carlo Boldrini, Carlo Fontanelli e Carlo Peri. Cfr. R. Fucini, Acqua passata, cit., pp. 516-517: «Ogni anno, per S. Carlo, i signori Peruzzi davano un pranzo agli amici di famiglia che si chiamavano Carlo. Di straforo ero qualche volta invitato anch'io».

7. Sulla nascita della «Nazione» (1859), il cui primo direttore fu Alessandro D’Ancona, si veda G. Spadolini, Firenze capitale, cit., pp. 245-248. Augusto Barazzuoli (Monticano [Siena] 1830-Roma 1894). Ultimo esponente di spicco, dopo la scomparsa di Ricasoli prima e di Peruzzi poi, della potente "consorteria" toscana e avvocato di fama, nonché direttore della «Nazione», fu chiamato da Crispi a reggere il dicastero dell'Agricoltura, Industria e Commercio. Corrado Tommasi-Crudeli (Pieve Santo Stefano [Arezzo] 1834-Roma 1900). Laureatosi in medicina, fu per alcuni anni professore universitario. Partecipò alla campagna del 1859 nei Cacciatori delle Alpi e, l'anno successivo, alla spedizione dei Mille. Fu membro del Consiglio superiore della Pubblica Istruzione e direttore del «Corriere Italiano». Venne nominato senatore nel 1892. La Peruzzi fa riferimento al Discorso dell'on. Tommasi Crudeli, in cui il Barazzuoli risponde alle accuse rivolte a Ubaldino Peruzzi circa la caduta della Destra Storica, pubblicato sulla «Nazione» tra il 2 e il 4 novembre 1876.

LXXV. Dall'aut. in BRF (FRF, cass. 8, ins. 20). Cart. post.: «All' Ing.e Renato Fucini / Empoli / per Vinci". Ann. aut. di Fucini: "Del Bello / Gotti / Momo / Gigi».

1. Annotazione aut. del Fucini.

2. Nel 1876 i Peruzzi si spinsero fino a Costantinopoli e al Bosforo. Cfr. M. P. Cuccoli, Emilia Toscanelli Peruzzi, cit., p. 201. 
smuoverla? Spero vederla in questo mese e già fin d'ora le rammento che il 4 Novembre è $S$. Carlo, ch' Ella è qui atteso a pranzo e che le converrà venire pronto a rallegrarci con qualche suo verso - i fiori dei poeti. Saluti da tutti e scriva.

E. P.

\section{LXXVI}

\section{R. Fucini a E. PeruZzi}

Sono in Firenze fino da stamattina. Ho scritto [?] il bigliettino al Roster e le rispondo io per dirle che con tanto piacere verrò Domenica all'Antella ${ }^{1}$. Anzi sono venuto a Firenze apposta.

Pensi a scansare il 13 e gradisca i cordiali saluti del suo devotissimo

R. Fucini

Da questa scelleratissima musa però non è stato possibile avere un pronunciamento carlista ${ }^{2}$.

\section{LXXVII}

R. Fucini a E. Peruzzi

Gentil.ma Sig. ${ }^{a}$ Emilia

Firenze 4 Decembre 1876

Prima di tutto le mando un saluto cordiale e dopo mi faccio ardito di raccomandare alla sua attenzione un mio gracile bozzettino in prosa che vedrà la luce nel prossimo fascicolo della Nuova Antologia ${ }^{1}$.

LXXVI. Dall'aut. In BNCF (CEP, cass. 74, ins. 11, n. 16). Bigl. vis. intestato "ING. Renato FuCINI". Data, non aut. e a lapis: 3 nov 76. Inedito.

1.Su Giorgio Roster si veda la nota 2 alla lettera XIV.

2. Cfr. la lettera precedente.

LXXVII. Dall'aut. in BNCF (CEP, cass. 74, ins. 11,n. 17). Lettera, 2 ff. su 1 c. Parzialmente edita in R. Melis, Una "babelica natura": Sidney Sonnino, Emilia Peruzzi e il problema della lingua a Firenze dopo l'unità, cit., p. 25.

1. Si tratta de il Matto delle giuncaie. Bozzetto padulano, pubblicato nel dicembre $1876 \mathrm{su}$ «La Nuova Antologia», pp. 165-773. Per quanto riguarda la rivista, fondata nel 1866 a Firenze da Francesco Protonotari (direttore fino al 1888) come mensile edito da Le Monnier, si vedano L. FedERZoni, Dalla vecchia alla novissima "Antologia", in L. Barbieri, Indici per autori e materie della Nuova Antologia dal 1866 al 1930, Firenze, Le Monnier, 1988, pp. VII-XXIII; M.M. Augello, La «Nuova Antologia» e la diffusione dell'econnomia politica (1866-1880), in Le riviste di economia in Italia (1700-1900), a cura di M.M. Augello, M. Bianchini, M.E.L. Guidi, cit., pp. 311-338. 
Si rammenta d'una certa sera nella quale l'Uzielli ed io restammo costassù rinchiusi dalla neve ${ }^{2}$ ? Cotesta sera, se ne rammenterà di certo, mi arrabattai tanto per mettere insieme la prefazione ai famigerati cento sonetti ${ }^{3}$ e dopo molte prove riuscite una peggio dell'altra ella mi disse e con tanta verità: - caro Fucini, quando scrive in versi ella è concettoso e vivace, ma nella prosa non la riconosco -. Ella diceva parole d'oro ed io ne tenni conto perché restai mortificato ${ }^{4}$.

Ora guardi se ho fatto nessun progresso e me ne dica una parola.

Mi rammenti al Sig. Ubaldino, gli domandi se ha scritto o se ha parlato col Ministro Coppino nel mio interesse e mi creda suo devot.mo

R. Fucini

\section{LXXVIII}

\section{E. PeruZZi a R. Fucini}

Grande trionfo. Il bozzetto è piaciuto immensamente. Lo dicono tutti una perla. Bravo!

Domani Mercoledì venga da me a casa in città fra le 3 e le 4 1/2.

12 Martedì.

\section{LXXIX}

\section{R. Fucini a E. PeruZZI}

Terrò conto dei suoi buoni ed amorevoli suggerimenti, per modo che ella in seguito sarà contenta di me. S'accerti però che non è pravità d'animo che mi fa scrivere certe

2. Gustavo Uzielli (Livorno 1839-Impruneta [Firenze] 1911). Studiò ingegneria a Pisa, interrompendo gli studi per correre alle armi durante la seconda guerra d'indipendenza. Nel 1860 partì con la spedizione Medici alla volta della Sicilia.. Laureatosi, insegnò mineralogia e geologia all'università di Modena e alla Scuola d'applicazione per ingegneri di Torino (1880-1881). Abbandonata la cattedra, tornò a Firenze per dedicarsi ai suoi studi. Nel 1896 ottenne la cattedra di geologia all'università di Parma. Il suo nome resta legato alla Società Geografica, nata per iniziativa sua, di Cristoforo Negri e di Cesare Correnti.

3. Si tratta della prefazione a R. FuCINI, Cento soneti in vernacolo pisano, Firenze, Pellas, 1872.

4. Lepisodio è ricordato anche in R. FucINI, Acqua passata, cit., pp. 511-512: «[...] fu amichevolmente osservato e deplorato che io, così fluido nello scrivere versi, stentassi tanto con la prosa e la scrivessi male. Me n'andai a letto con una spina nel cuore e nell'amor proprio; e mi proposi di arrivare a scrivere degnamente anche in prosa».

LXXVIII. Dall'aut. in BRF (FRF, cass. 8, ins.19). Lettera intestata "EP" e listata a lutto, 1f. su 2 cc. Data, non aut. e a lapis: 12/8/76. Inedita.

1. Si tratta probabilmente del Matto delle giuncaie. In questo caso, la lettera della Peruzzi costituirebbe la risposta alla precedente di Fucini e quindi sarebbe stata inviata non nell'agosto, ma nel dicembre del 1876.

LXXIX. Dall'aut. in BNCF (CEP, cass. 74, ins. 11, n. 18). Bigl. vis. Inedito. 
cose ma quella beata inconsideratezza per la quale non temo guai allorché il ridicolo mi si presenta sotto qualunque forma e da qualunque parte mi giunga.

Torno a pregarla di domandare al Sig. Ubaldino se ha parlato o scritto di me al ministro Coppino essendo questa cosa troppo vitale per la mia esistenza. Senza entrare in particolari e senza tanti preamboli le dirò che senza una occupazione onestamente lucrosa io sarò costretto fra pochi mesi o alla più lunga fra un anno a lasciar Firenze e ritirarmi in campagna. Per me individualmente prenderò la cosa in santa pace ma per la mia famiglia e specialmente per le mie care piccine sono addoloratissimo.

Mi perdoni queste lamentazioni ma ritenga per vero, molto vero quello che le ho detto e le lascio considerare se lo stato d'animo mio è desolato.

Gradisca un saluto affettuoso del suo devotissimo

9 Decembre 1876

R. Fucini

\section{LXXX}

E. Peruzzi a R. Fucini

Antella 15 Decembre 76

Ubaldino approva che andiate da lui Lunedì ${ }^{1}$. Quanto all'ora sarà bene che Ella passi verso le 11 al Municipio per far domandare a che ora Ubaldino sarà libero di vedervi.

Ciò che Ella mi dice mi rallegra e se il Gotti spera sta bene che si speri anche noi ${ }^{2}$.

Studii il modo di non perdere studiando la sua spontaneità.

La gita all'Antella preferibilmente la faccia o il Sabato sera o le Domeniche mattina e sera... o i giorni di Natale o del po dell'anno. Mille cose belle .

Emilia

\section{LXXXI}

\section{R. Fucini a E. PeruZzi}

Tanti auguri e cordiali felicitazioni. L'abboccamento domandato dal Sig. Ubaldino ci fu accordato e glie ne sono molto riconoscente. La colpa non è di nessuno ma il

LXXX. Dall'aut. in BRF (FRF, cass. 8, ins. 19). Lettera intestata "EP" e listata a lutto, 3 ff. su 2 cc. Sul retro, ann. a lapis di Fucini: «Peruzzi». Inedita.

1.Fucini aveva richiesto più volte l'aiuto dei Peruzzi per trovare un'occupazione, dopo che il tentativo di vivere solo del lavoro di letterato era fallito. Cfr. le lettere LXXI, LXXVII e LXXIX.

2. Si veda la lettera LXX.

LXXXI. Dall'aut. in BNCF (CEP, cass. 74, ins.11, n. 19). Bigl. vis. Inedito. 
risultato fu abbastanza desolante, in quantoché le mie deboli speranze si convertirono in fortissimi dubbi. Speriamo bene.

\title{
Suo devotissimo
}

25 Dicembre 1876

\author{
R. Fucini
}

\section{LXXXII}

R. Fucini a E. PeruZZI

Grazie, grazie, grazie ${ }^{1}$. Terrò conto dei suoi buoni suggerimenti.

A rivederci a Giugno all'Antella; intanto salute, salute, salute.

24 Aprile 1877

R. Fucini

\section{LXXXIII}

E. PeruZzi a R. Fucini

\section{Antella 5 Giugno 77}

Mi scrive lo Zumbini da Napoli, caro Fucini, che Ella deve essere arrivato ed avrà infinite cose da narrare ${ }^{1}$. Desidero rivederla e La prego dirmi se è a Firenze e quando potrebbe recarsi all'Antella. Napoli è città divertentissima e sono certa che le sue impressioni saranno state molte e vive.

Aff.ma

Emilia

Ubaldino ha sofferto per una tosse tenace ma sta meglio.

LXXXII. Dall'aut. in BNCF (CEP, cass. 74, ins. 11, n. 20). Bigl. vis. Inedito.

1. Per interessamento della signora Emilia, Fucini aveva ricevuto l'incarico da Pasquale Villari di recarsi a Napoli, dove ebbe come guida il giovane Giustino Fortunato. Cfr. E. BENUCCI, Introduzione a E. DE AMICIS, Un salotto fiorentino del secolo scorso, cit., p. 53.

LXXXIII. Dall'aut. in BRF (FRF, cass. 8 ins. 19). Lettera intestata "EP”, 2 ff. su 2 cc. Inedita.

1. Bonaventura Zumbini (Pietrafitta [Cosenza] 1836-Bellavista [Napoli] 1916). Si avviò alla carriera dell'insegnamento prima a Cosenza, poi a Napoli, dove si era trasferito nel 1868. Nel 1878 ottenne la cattedra di letteratura italiana all'università di Napoli, succedendo a Luigi Settembrini. Nel 1905 venne nominato senatore. 


\section{LXXXIV}

\section{R. Fucini a E. PeruZzi}

Gentil.ma Sig. ${ }^{a}$ Emilia

Dianella 12 Giugno 1877

Reduce dal mio bellissimo viaggio, in settimana tornerò a Firenze traboccante di impressioni di ogni genere e lieto di riposarmi fra le carezze della famiglia e degli amici ${ }^{1}$.

Da Napoli non le ho scritto perché mi era proposto di non farlo con nessuno meno che per grandissima urgenza per non togliere neanche una mezz'ora al tempo che avevo scrupolosamente misurato per vedere ed osservare tutto il meglio che vi era da osservare e da vedere, ma appena rientrato negli ozii di Capua il mio pensiero è ritornato ai miei buoni amici e a Lei, fra i primi, mando un saluto affettuoso accompagnato dal desiderio che il Sig. Ubaldino si sia rimesso dai suoi leggeri incomodi dei quali ho avuto notizia dai fogli pubblici.

Nel ritorno mi son fermato all'Elba ${ }^{2}$ dove sono stato ospitato, si può immaginar come, dalla Sig.a Vittorina che ho trovata assai di buonumore perché Bistino stà così bene che $^{3}$, all'apparenza almeno, se non sembra affatto ristabilito, mi sembra lasci dubbio alcuno sui buoni resultati finali della penosa e lunghissima cura di sacrifizi che da tanto tempo quel povero ragazzo si è imposta.

Di Napoli parleremo a voce, intanto la saluta con amichevole rispetto il suo devot.mo

R. Fucini

P.S: Le anticipo i complimenti caldissimi di Zumbini e di Fortunato ${ }^{4}$.

LXXXIV. Dall'aut. in BNCF (CEP, cass. 74, ins. 12, n. 1). Lettera, 3 ff. su 2 cc. Inedita.

1. Si tratta del viaggio a Napoli a cui si fa riferimento nella lettera precedente.

2. Cfr. R. Fucini, Acqua passata, cit., p. 536: «Tornando da Napoli, sbarcai a Livorno, e lì, montato in battello, filai all'isola d'Elba dove avevo amici e parenti».

3. Su Giovan Battista Giorgini cfr. la nota 3 alla lettera LXVIII.

4. Giustino Fortunato (Rionero in Volturne [Potenza] 1848-Napoli 1932). Laureatosi nel 1869 in legge, cominciò a collaborare con vari giornali moderati, quali «La Patria» e «L'Unità nazionale»; dal 1878, per indicazione di Pasquale Villari, divenne corrispondente della "Rassegna Settimanale», pubblicandovi numerosi contributi critici che vertevano principalmente sull'economia di Napoli. Eletto nel 1880, appoggiò la politica di Depretis, ma non fece altrettanto con quella crispina. In collaborazione con Salvemini fondò il settimanale «L'Unità. Problemi della vita italiana». In occasione della prima guerra mondiale si schierò sul fronte neutralista. Visse l'avvento del fascismo come conferma delle sue previsioni più pessimistiche e ne fu, perciò, subito deciso avversario. 


\section{LXXXV}

R. FuCini a E. PERUZZI

Gentil.ma Sig. Emilia

A pranzo è impossibile che venga e la ringrazio di tanta gentilezza. Salirò a salutarla più tardi con la diligenza. Mille complimenti

R. Fucini

\section{LXXXVI}

R. FuCini a E. PeruZZi

Gentil.ma Sig. ${ }^{\text {a }}$ Emilia

Dianella 10 Ottobre 1877

Finalmente ho trovato da occuparmi e sono contento. Fino dal 6 corrente mi è stata partecipata la nomina di insegnante Lingua e Letteratura italiana nelle Scuole Tecniche di Pistoia e fra pochi giorni mi recherò in quella Città perché il $1^{\circ}$ di Novembre dovrò entrare nella nuova carica ${ }^{1}$.

Mi dispiace di lasciare gli amici e Firenze, ma chi ci ha che fare? Ella che sa benissimo come stavano le mie cose in Firenze, saprà anche compatirmi se per dar da vivere alla mia famiglia ho preso questa risoluzione.

Nel corso dell'invernata spero che ci vedremo spesso perché ogni tanto verrò ad abbracciare i miei cari che fino a primavera non condurrò a Pistoia avendo ormai pagata la pigione del mio quartiere in Piazza D’Azeglio per tutto questo semestre.

Gradisca i saluti rispettosi del suo devot.mo amico

R. Fucini

LXXXV. Dall'aut. in BNCF (CEP, cass. 74, ins. 12, n. 2). Bigl. vis. Data, non aut. e a lapis: 19.7.77. Inedito.

LXXXVI. Dall'aut. in BNCF (CEP, cass. 74, ins. 12, n. 3). Lettera, 2 ff. su 1 c. Inedita.

1. Fucini aveva trovato questo impiego grazie a Giovanni Procacci. Cfr. L.G. SBrocCHI, Renato Fucini. L'uomo e l'opera, cit., p. 24. 


\title{
LXXXVII
}

\author{
E. Peruzzi a R. Fucini
}

Antella Lunedì

La notizia che Ella mi diede ci afflisse per il dispiacere di vederla allontanarsi da noi e ci consolò perché da a Lei una occupazione onorevole ${ }^{1}$. Sappia che appunto avevo scritto per Lei sia come Insegnante, sia come correttore alla Gazzetta d'Italia, ma ormai il posto è trovato e la vicinanza è ottima cosa per Lei e per gli amici suoi. Si avvicina il giorno di S. Carlo e se Le è possibile venire anche qui a pranzo come gli anni scorsi - è appunto una Domenica di Novembre, cosicché Ella può esser libero ${ }^{2}$.

Ad ogni modo venendo o non venendo mi mandi almeno un Sonetto pei 4 Carli che Ella $\mathrm{sa}^{3}$. Ma faccia di venire. Vi sarà l'ultima gita che Consorzio troverà tutti gli amici e gli amici saranno tristissimi di trovare Lei. Non mi trovi senza che non gliela meniamo buona e l'aspettiamo senza fallo.

Mi risponda e mi dica un bel Sì. Ho parlato di Lei e l'ho molto raccomandato al tenente Grifi marito di una Altoviti che stà a Pistoja e potrà darle alla penna molte notizie utili a sapersi. Le dico all'orecchio che il Manfroni ${ }^{4}$, Ispettore Scolastico del Circondario di Pistoja crede che ci voglia un carattere d'oro e una grande abilità per vivere in pace a Pistoja con i Pistoiesi. Sono un po' come i Guelfi e i Ghibellini. Di tutto questo parleremo a voce. Aspetto lettere Sue e poi più tardi Lei e un Sonetto.

Aff.ma

Emilia

\section{LXXXVIII}

\section{R. Fucini a E. PeruZZi}

Gentilissima Sig. Emilia

Dianella 30 Ottobre 1877

La risposta al suo gentile invito dipendeva da quello che mi si sarebbe detto in una lettera che aspettavo da Pistoia. Questa lettera finalmente è arrivata e senza

LXXXVII. Dall'aut. in BRF (FRF, cass. 8, ins. 19). Lettera intestata "EP”, 4 ff. su 2 cc. Anno, non aut. e a lapis: 1877 . Inedita.

1. Si riferisce a quanto detto da Fucini nella lettera precedente.

2. Sulla festa dei Carli si veda la nota 6 alla lettera LXXIv.

3. Cfr. ancora la nota 6 alla lettera LXxIv.

4. Su Mario Manfroni si veda Introduzione, p. 16, nota 45.

LXXXVIII. Dall'aut. in BNCF (CEP, cass. 74, ins. 12, n. 4). Lettera, 4 ff. su 2 cc. Inedita. 
annunziarmi il giorno preciso nel quale dovrò portarmi là, $\mathrm{mi}^{1}$ dice che stia pronto a partire perché da un momento all'altro potrò esser chiamato.

Stando così le cose, non posso né voglio mettermi fuor di tiro neanche in giorno festivo perché dovendovi essere la distribuzione dei premj o diplomi, salvo il vero ${ }^{2}$, molto probabilmente sarà fatta in giorno festivo. La prego, nel dubbio, a tenermi per disimpegnato, ed a scusarmi.

La ringrazio dei buoni consigli che $\mathrm{mi}$ da. Conosco Pistoia molto bene, e so che là, come in tutto il resto del mondo, vi sono Guelfi e Ghibellini; Bianchi e Neri ma i Ghibellini e i Bianchi in quel Paese sono i più ${ }^{3}$.

Io, dopo aver portato laggiù le mie tende, m'occuperò molto dei fatti miei, della mia famiglia e de' miei studj; ma nel caso che sia costretto a tirar fuori la testa anch'io, credo che quel po' d'odore che ho di Ghibellino e di Bianco non mi debba nuocere laggiù e spero di potermici trovar bene.

Saluti i Carli e invece del sonetto che in questo momento non saprei fare nemmeno a torturarmi il cervello perché ho troppe preoccupazioni, faccia loro un brindisi secondo la mia intenzione e creda sempre alla rispettosa amicizia del suo devot.mo

R. Fucini

P.S: Una imperdonabile dimenticanza mi accorgo d'aver commesso con la furia e le faccio le mie più grandi scuse. Ho dimenticato di ringraziarla delle premure che ultimamente si è data per trovarmi una occupazione in Firenze. La ringrazio di grandissimo cuore perché con quel pensiero ha toccato la corda più sensibile dell'animo mio, ma ormai è finita. Il giorno che salutai Pistoia avevo già detto addio a Firenze.

\section{LXXXIX}

\section{R. Fucini a E. PeruZzi}

Gentil.ma Sig. ${ }^{a}$ Emilia

11 Novembre 77 Firenze

Tutti i proponimenti che avevo fatto per questi ultimi mesi che ho da passare per metà a Firenze, sono stati buttati all'aria dalla fatica e dallo strapazzo che, almeno sul

\footnotetext{
1. Corregge «si».

2. Cassato «che».

3. Era definito "partito nero" l'opposizione clericale, mentre col nome di Bianchi si indicavano i moderati. Cfr. H. UlLRICH, Sistemi elettorali e sistema politico: dalla riforma del 1882 alla crisi di fine secolo, in Idee di rappresentanza e sistemi elettorali in Italia tra Otto e Novecento. Atti della terza giornata di studio "Luigi Luzzatti" per la storia dell'Italia contemporanea (Venezia, 17 novembre 1995), a cura di P.L. Ballini, Venezia, Istituto veneto di scienze, lettere ed arti, 1997, p.92.

LXXXIX. Dall'aut. in BNCF (CEP, cass. 74, ins. 12, n. 5). Lettera, 3 ff. su 2 cc. Ann. (aut. della Peruzzi?) a lapis sulla quarta facciata: «Fucini». Inedita.
} 
principio, mi costa la mia vita nuova. Credevo che il trovarmi ogni giorno in Firenze, libero dalle 3 in là, mi permettesse di correre a salutare i miei amici e invece sento che non m’è possibile. L'Antella dei Peruzzi, il Montepiano dei Pellizzari e la Concezione dei Sabatier son diventati lontani, lontani, lontani ${ }^{1}$.

Ogni mattina mi alzo alle 6, corro alla stazione e di lì a Pistoia dove appena arrivato, senza un respiro, debbo fare tutti i giorni tre e a volte quattro ore di lezione a 150 speranze d'Italia divise in tre classi; appena ho finito, di corsa al vapore e via a Firenze. Arrivato a Firenze alle 2 ho da studiare, da prepararmi alle lezioni del giorno dopo, ho da riguardare componimenti, ho da arrabattarmi, in somma, come un cane tanto più che mi è saltata addosso anco la fisima di scrivere e pubblicare il mio viaggio a Napoli ${ }^{2}$. Pensi a molte altre preoccupazioni che debbono molestarmi in un momento così critico; pensi che dopo tanta fatica di muscoli e di mente, l'orizzonte che mi si presenta la sera è quello di riprincipiare il giorno dopo alle sei eppoi mi scusi e mi compatisca, sig. Emilia, se invece di venire a salutarla in persona lo faccio con queste righe. Mi scusi perché me lo merito.

A Pistoia sono stato accolto con molta festa e questo ha molto contribuito a farmi guardare con più calma l'idea d'andarmi a seppellire in provincia e a mettermi un po' in rialzo il morale, ma ho avuto dei gran giorni neri. Basta, non voglio rattristarla e mi cheto.

La saluto rispettosamente e mi creda suo devot.mo

R. Fucini

XC

\section{R. Fucini a E. PeruzZi}

Gentil.ma Sig. Emilia

17 Settembre 1877

Non ho mai dubitato della sua preziosa amicizia né di quella del Sig. Ubaldino; sarei un imbecille o un ingrato. Dubito soltanto che ella creda interamente alla tristezza delle circostanze che mi forzano a ritirarmi in campagna. In due anni ho guadagnato

1. Sulla villa dell'Antella cfr. la nota 1 alla lettera I. Su Montepiano si veda la nota 1 alla lettera LX. L'elegante residenza dei Sabatier prende il nome da un monastero vicino ed è situata nei pressi di Fiesole. François Sabatier, suocero di Michele Amari, era un uomo di grande cultura, appassionato di arte e storia. Compì numerosi viaggi in vari paesi europei e tradusse Guglielmo Tell di Schiller e Faust di Goethe. Era sposato con la cantante ungherese Carolina Hungher, scelta da Beethoven per la prima esecuzione della nona sinfonia. Cfr. T. SCHARTEn, Les voyages et séjours de Michelet en Italie. Amitiés italiennes, Paris, Droz, 1934, p. 155. Si veda inoltre la descrizione che il Fucini dà del Sabatier in Acqua passata, cit., pp. 496-498: «[...] onesta e simpatica persona, era un francese, un francese di Grenoble, della più bell'acqua, piccolo, grasso, tondo, rabbuffato nei capelli e nella barba, il quale, dalla più dolce calma passava improvvisamente alle furie d'Oreste».

2. Si tratta di Napoli a occhio nudo, pubblicato a Firenze dalla casa editrice Le Monnier nel 1878.

XC. Dall'aut. in BNCF (CEP, cass. 74, ins. 12, n. 6). Bigl. vis. Inedito. 
30 lire spendendone settemila di mio e ora sono esausto. Firenze è una sirena e io ne sono innamorato ma non fino a morire di marasmo per lei. Non giudichi dal mio buonumore; è una vernice che mi ha regalata la Provvidenza per non diventare odioso a me ed agli altri.

Domani verrò volentieri all'Antella ed ora vado dal D'Ancona per fissare ${ }^{1}$.

Intanto la ringrazia e la saluta il suo devot.mo

R. Fucini

\section{$\mathrm{XCI}$}

R. FuCini a E. PERUZZI

Gentilissima Sig. Emilia

Firenze 12 Decembre 1877

Ser Brancadoria non è morto unquamdie [?]

E mangia e beve e dorme e veste panni.

Un saluto di core dopo tanto tempo. E quando il suo ritorno in città? Desidero tanto rivederla. Ma quante peripezie quella povera Sig. ${ }^{a}$ Vittorina ${ }^{1}$ ! E le sue virtù non meriterebbero da vero così triste ricompensa. Io seguito sempre la mia vita ventilata andandomene continuamente in su e in giù tra Firenze e Pistoia; è una vita da disperati che a buona stagione cesserà, ma fortunatamente sto bene di salute e sono allegro e contento.

Conobbi il Tigri ${ }^{2}$ con tanto piacere e parlammo molto di Lei ${ }^{3}$. $\mathrm{E}$ un buon vecchietto del quale si è impadronito il tempo e presto ce lo porterà via. Eppoi toccherà anche a

1. Alessandro D’Ancona (Pisa 1835-Firenze 1914). Si iscrisse nel 1859 alla facoltà di giurisprudenza di Torino, ma ben presto abbracciò lo studio delle lettere, diventando uno dei più illustri filologi italiani. Tra il 1859 e il 1860 diresse «La Nazione» e, nello stesso 1860, ottenne la cattedra di letteratura italiana all'università di Pisa. Dal 1900 al 1909, sempre a Pisa, ebbe l'incarico di esegesi dantesca. Collaborò alla «Nuova Antologia» e alla "Rassegna Settimanale». Nominato senatore nel 1904, fu sindaco di Pisa nel 1906 e nel 1907. Potrebbe tuttavia trattarsi anche di Sansone D’Ancona (Pesaro 1814-Firenze 1894). Laureatosi a Pisa in scienze matematiche, collaborò con «La Nazione». Nel 1859 fu inviato dal governo provvisorio in Inghilterra e in Francia per compiere delle missioni finanziarie. Appena eletto deputato, Cavour gli offrì la carica di direttore delle Finanze; in seguito venne nominato sopraintendente delle Finanze.

XCI. Dall'aut. in BNCF (CEP, cass. 74, ins. 12, n. 7). Lettera, 2 ff. su 1 c. Inedita.

1. Si tratta di Vittoria Manzoni, figlia del celebre scrittore e moglie di Giovan Battista Giorgini.

2. Cassato «g».

3. Giuseppe Tigri (Pistoia 1806-1882). Frequentò il seminario vescovile di Pistoia, prendendo i voti. Nel 1837 insegnò retorica ed eloquenza nel Collegio Cicognini di Prato, dove ebbe fra i suoi allievi anche Ubaldino Peruzzi. Animato da sentimenti liberali, fu in corrispondenza col Guerrazzi; tra il 1847 e il 
noi; eppoi a quelli dopo di noi; e poi a quegli altri e così via fino alla consumazione dei secoli, et per omnia saecula saeculorum. Amen. E la saluto e la reverisco con stima e rispettosa amicizia

R. Fucini

\section{XCII}

\section{R. Fucini a E. PeruZZi}

Gentil.ma Sig. Emilia

Non sto punto meglio, tanto è vero che la persona incaricata di portarle questo biglietto ha pure la commissione di prendere un senapismo che voglio mettermi sul petto per vedere di pormi in grado d'andare anche domattina a spezzare il pane della sapienza a' miei ragazzi. È una pessima giornata, ed io la vedrò attraverso ai cristalli della finestra. Ha veduto il capitolo sul Camposanto vecchio di Napoli ${ }^{1}$ ? Che le ne è parso? Non è dei migliori, ma fu prescelto per la sua brevità. Quello che lì racconto l'ho visto tutto da me co' miei occhi. Nel corpo del libretto ne sentirà molte delle peggio.

Oggi il Pareto sarà da $\mathrm{Lei}^{2}$. L'avrei salutato volentieri ma non potendolo fare in persona ne incarico la di Lei gentilezza.

La ringrazio e la saluto rispettosamente

\section{Suo devot.mo}

R. Fucini

\section{Domenica}

1849 compose poesie politiche. Dopo aver ricoperto il ruolo di ispettore straordinario in Sardegna e di provveditore agli studi in Sicilia, fu ispettore scolastico nella città natale. Nel 1853 divenne bibliotecario della Biblioteca Forteguerri di Pistoia.

XCII. Dall'aut. in BNCF (CEP, cass. 74, ins. 12, n. 8). Bigl. vis. Data, non aut. e a lapis: 17.3.78. Inedito.

1. Si tratta di una delle lettere da cui è composto Napoli a occhio nudo. Venne pubblicata sulla «Rassegna Settimanale», vol. I (24 febbraio 1878), n. 8, pp. 134-136.

2. Vilfredo Pareto ( Parigi 1848 - Céligny [Ginevra] 1923). Laureatosi ingegnere a Torino nel 1869, dopo poco diventò direttore della ferrovia di S. Giovanni Val d'Arno, poi direttore generale delle Ferriere Italiane. Il lavoro alle ferriere gli fornì la possibilità di compiere numerosi viaggi all'estero, in particolare in Inghilterra. In questi anni scese in campo a favore del liberoscambismo, denunciando la povertà concettuale del protezionismo con vivaci articoli polemici, ripudiati in età matura. Dal 1893 al 1906 insegnò economia politica all'università di Losanna; nel 1923 fu nominato senatore e gli venne offerto l'incarico, rifiutato per motivi di salute, di rappresentare l'Italia alla Società delle Nazioni. La bibliografia su Vilfredo Pareto è piuttosto vasta; ci limitiamo perciò a segnalare alcuni tra i saggi più recenti: G. CoLosimo, Introduzione a Vilfredo Pareto economista, Napoli, Stabilimento Tipografico Genovese, 1968; L. Avagliano, Vilfredo Pareto. Lo sviluppo economico italiano, Salerno, Società editrice salernitana, 1975; S. Borgia, Politica e potere in V. Pareto, Lecce, Quaderno di Ethos, 1984; P. BonetTI, Il pensiero politico di Pareto, Bari, Laterza, 1994; La ragione e i sentimenti. Vilfredo Pareto e la sociologia, a cura di E. Rutigliano, 


\title{
XCIII
}

\section{E. PeruzZi a R. Fucini}

Mille e mille ringraziamenti per il caro e bel libro ${ }^{1}$. Venga a vederci Sabato e mi dica se Domenica puo $^{2}$ desinare da noi.

Voglio che legga le bellissime lettere del bravo Mantellini Deputato di Firenze ${ }^{3}$. Sono col Corriere d'jeri e di stamane - nella Nazione d'jeri e stamane nella Vedetta le abbia tutte e tre (l'ultima verrà domani) e le faccia leggere a quanti più puo ${ }^{4}$ anche a Pistoja ${ }^{5}$. La verità scappa fuori che è impossibile non essere convinti.

Aff.

Emilia

\section{XCIV}

\author{
R. Fucini a E. PeruZzi
}

Gentilissima Sig. Emilia

Pistoia 8 Maggio 1878

Eccomi da dieci giorni cittadino pistoiese. Volevo congedarmi venendo da Lei in persona a salutarla, ma la tempesta del cielo che minacciò tanto la mia povera roba durante i giorni dello sgombero, suscitò anche una tale tempesta nella parte meno

Milano, Angeli, 1994; L. Bruni, Vilfredo Pareto. Alle radici della scienza economica del Novecento, Firenze, Polistampa, 1999; Economia, sociologia e politica nell'opera di Vilfredo Pareto, a cura di C. Malandrino, R. Marchionatti, Firenze, Olschki, 2000; Vilfredo Pareto (1848-1923). L'uomo e lo scienziato, a cura di G. Manca, Milano, Libri Scheiwiller, 2002.

XCIII. Dall'aut. in BRF (FRF, cass. 8, ins. 19). Bigl. vis. Sulla busta: «Ill.mo Sig. Ing.re Renato Fucini / 14 Piazza D’Azeglio». Data ricavata dal riferimento alle lettere di Giuseppe Mantellini: 14 aprile 78 . Inedito.

1. Si tratta di Napoli a occhio nudo: lettere ad un amico, edito in quel torno di tempo da Le Monnier.

2. Così nell'originale.

3. Giuseppe Mantellini (Firenze 1816-Roma 1885). Compiuti gli studi di legge, esercitò brillantemente la professione a Firenze. Eletto deputato alla Camera, fece parte di numerose commissioni e fu spesso relatore di importanti disegni di legge. Nominato consigliere di Stato, gli venne affidato il compito di avvocato erariale

4. Così nell'originale.

5. Si tratta delle tre lettere scritte dal Mantellini al direttore del giornale «Opinione Nazionale» circa la questione dei debiti del Comune di Firenze e pubblicate sia dalla «Nazione» (10, 11 e 12 aprile 1878), che dal "Corriere Italiano» (10, 11, 12 e 13 aprile 1878). «La Vedetta», invece, riporta esclusivamente la notizia della pubblicazione delle lettere da parte dell' «Opinione Nazionale» (14 aprile 1878).

XCIV. Dall'aut. in BNCF (CEP, cass. 74, ins. 12, n. 9). Lettera, 4 ff. su 2 cc. Inedita. 
burrascosa del mio organismo che non ebbi né capo, né tempo di mandare ad effetto molti miei proponimenti fra i quali quello di abbracciare tanti buoni amici dai quali ho dovuto allontanarmi insalutato ospite.

La distanza, però, fra Pistoia e Firenze non è tale da impedirmi di rivederli tutti, e presto lo farò appena mi sarò pacificamente sistemato nel mio nuovo domicilio.

Il Roster le avrà fatto molti miei saluti e scuse e la avrà anche fatto il quadro delle smanie di questo innocente chiocciolone che attraverso all'acqua, ai fulmini ed al vento ha dovuto strascicarsi dietro fin quaggiù il suo incomodo guscio formato da quattro grosse barrocciate di mobilia ${ }^{1}$.

La quiete di questa piccola città mi ha sedotto e spero bene per i miei studj se la pesca, le passeggiate e la caccia non mi faranno troppo campagnolo, delle quali conseguenze ho molta ${ }^{2}$ ragione di temere perché le mie tendenze e i miei sospiri sono andati sempre da quella parte anche in mezzo alle glorie cittadine e perché è troppo vero quel proverbio che dice: il lupo perde il pelo ma il vizio, mai. Staremo a vedere.

Il mio libretto ha fatto rumore simpatico a Napoli, a Milano e in altre città d'Italia e ne sono soddisfatto ${ }^{3}$. A Firenze soltanto nessuno se ne è occupato. Pare che gli aristarchi d'Atene siano al quanto volubili nei loro amori. Facciano pure il loro comodo.

Le mie occupazioni quaggiù sonno leggiere e piacevoli, l'aria di questo paese eccellente, la campagna maravigliosa e per conseguenza ottima la salute della mia famiglia, e questo fatto mi imbottisce talmente di consolazione tutti i cantucci del core che se dicessi d'essere scontento del mio nuovo stato, sarei un impudente mentitore.

Dunque, allegri! Perché tirata la somma, ella vede che il mio bando l'ho preso con bastante tranquillità, che sto bene e che la ferita toccata nell'amor proprio rimarginerà presto e tanto bene da non lasciare nemmeno ombra di cicatrice. La prego de' miei rispettosi complimenti al Sig. Ubaldino e di tanti affettuosi saluti ai nostri comuni amici, mentre mi ripeto suo devot.mo

R. Fucini

1. Su Roster si veda la nota 2 alla lettera XIV.

2. Cassato «da».

3. Si tratta di Napoli a occhio nudo. 


\section{Venerdì 17-78}

Domenica gita all'Incontro $^{1}$ - la presenza di lei raddoppierebbe il piacere a tutti. Partenza de Firenze al tocco - vada subito dal Vimercati o meglio in Piazza de'Giudici - partenza diligenza al tocco - preciso $^{2}$. La domenica ci pare che potrà venire - faccia l'impossibile. Senza l'amico Fucini tutti saremmo meno lieti.

E.

\section{XCVI}

E. PeruzZi A R. Fucini

Antella 29 Ott. 1878

Caro Fucini. Ella è proprio più lontano giacché non cerca nemmeno di avvicinarsi all'Antella scrivendo. Ma a Firenze ci viene mai? Quando ci viene? Ha letto il libro del Mari su Firenze ${ }^{1}$ ? Cosa ne pensa?

Per il S. Carlo $\left.4{ }^{[* * *}\right]$ verrà all'Antella quest'anno ${ }^{2}$ ? Mi scriva se per quel tempo si può trovare a Firenze e se potrebbe venir qua?

I viaggiatori stanno bene ed erano l'altro giorno a Napoli ora li aspettiamo di momento in momento ${ }^{3}$. Ubaldino le fa tanti saluti - gli amici la ricordano con affezione. Venga dunque e porti gran massa di sonetti, poesie e novelle inedite. Risponda subito, caro Fucini, e faccia di venire - con la Musa o senza Ella è sempre desiderato dai suoi fidi amici

Peruzzi

XCV. Dall'aut. in BRF (FRF, cass. 8, ins. 19). Cart. post. Ind.: «All'Ill.mo Sig.re R. Fucini / Pistoia». Data del timbro postale: 17-5-78.

1. Località nei pressi dell'Antella.

2. Piazza de' Giudici è una piazza di Firenze, che si affaccia sul Lungarno de' Medici, vicino al piazzale degli Uffizi. Su Guido Vimercati si veda la nota 5 alla lettera LXI.

XCVI. Dall'aut. in BRF (FRF, cass. 8, ins. 19). Lettera intestata "EP", 4 ff. su 2 cc. Inedita.

1. Adriano Mari (San Salvi [Firenze] 1813-Fiesole 1887). Nel 1859, dopo la cacciata del granduca, fu eletto all'assemblea toscana che votò l'annessione al Piemonte. Fu più volte presidente della Camera; nel 1867 venne ricoprì la carica di ministro della Giustizia. Il libro di cui parla la Peruzzi è La questione di Firenze (Firenze, Niccolai, 1878), in cui si tratta della situazione finanziaria del Comune. Sulla bancarotta del Comune di Firenze si veda S. Soldani, Emilia Toscanelli Peruzzi, o la passione della politica, cit., p. 25.

2. Sulla cena che i Peruzzi davano ogni anno per S. Carlo si veda la lettera LXXIv, nota 6.

3. Non sappiamo a chi la Peruzzi si riferisca. 
El pajarillo

Daba sustento a un pajarillo un día

Lucinda, y por los hierros del portillo

fuéssele de la jaula el pajarillo

al libre viento en que vivir solía.

Con un sospiro à la ocasión tardía

tendió la mano, y no pudiendo asillo

dijo (y de sus mejillas amarillo

volvió il clavel entre su nieve ardía).

¿Adonde vas por despreciár tu nido

$\mathrm{Al}$ peligro de ligas y de balas

$\mathrm{Y}$ el due o huyes que tu pico adora?

Oyóla il pajarillo enternecido

$\mathrm{Y}$ a la antigua prision volvió las alas

¿Que tanto puede una mujer que llora ${ }^{4}$ !

Questo Sonetto del Vega è scritto dal De Amicis una sera dopo avermelo recitato in casa Peruzzi ${ }^{5}$.

\section{XCVII}

\section{R. Fucini a E. PeruZZI}

Gentil.ma Sig. Emilia

Dianella $1^{\circ}$ Novembre 1878

Mi è giunta tanto gradita la sua lettera. La solitudine mi aggrava in tal modo la repugnanza a scriver lettere che non esito punto a confessarmi un perfetto egoista verso gli amici giacche $^{1}$ niente mi rallegra più della voce d'un amico lontano che si ricorda di me.

4. «Dava il becchime a un uccellino un giorno / Lucinda, ma attraverso lo sportello / fuor dalla gabbia volò via l'uccello, / bramando all'aria aperta far ritorno. // Tardi tese la mano, e per lo scorno / di non poter ghermire quel rubello, / sospira e dice (scolorando il bello / incarnato, di fuoco e neve adorno): // - Dov'è che vai, lungi da me, smarrito, / per esporti a pericoli mortali, / e disprezzi colei che ti ama tanto? - // La sentì l'uccellino e intenerito / all'antica prigione volse l'ali: / tale potere ha di una donna il pianto" (L. De Vega, Liriche, Torino, Einaudi, 1974, p. 125).

5. Data la vastità della bibliografia su Edmondo De Amicis (Oneglia 1846 - Bordighera 1908), ci limitiamo a segnalare alcuni tra i saggi più recenti: L. Gigli, De Amicis, cit.; I. SCARAmucCI, De Amicis, Brescia, La scuola, 1964; F. CONTORBIA, Edmondo De Amicis. Atti del convegno nazionale di studi (Imperia, 30 aprile-3 maggio 1981), Milano, Garzanti, 1985; B. TraversetTI, Introduzione a De Amicis, Bari, Laterza, 1991; E. Tоsтo, Edmondo De Amicis e la lingua italiana, Firenze, Olschki, 2003.

XCVII. Dall'aut. in BNCF (CEP, cass. 74, ins.12, n. 10). Lettera, 4 ff. su 2 cc. Inedita.

1. Così nell'originale. 
A Firenze sono stato due volte di passaggio per Pistoia e non ho mai incontrato un amico. Ho girellato per le sue strade nel più stretto incognito come un terrazzano calato a comprarsi il giubbone col pelo, ho fatto l'ora del treno e son ripartito.

Non ho letto il libro del Mari e lo leggerò volentieri perché il nome dell'autore me lo raccomanda anche troppo, ma la povera Firenze rialzata a suon d'argomentazioni mi fa pena e vorrei vedere uscir fuori da parte dei nostri cari fratelli che ce l'hanno così brutalmente rovinata ${ }^{2}$.

All'Antella per S. Carlo! Così potessi venire come ne avrei grande desiderio e invece, ad aggravarmi anche di più la privazione, quel giorno preciso sarò in cammino per ritornare alle mie occupazioni che ricominciano la mattina del 5. Spero in tempi migliori e intanto mi divago col mio cane e col mio fucile correndo dietro agli uccelli per fuggire alla noja che mi rincorre.

I nostri viaggiatori sono stati sfortunati. Nelle loro lettere hanno parlato soltanto delle maggiori contrarietà ed io credo che ben altre e non piccole né poche siano loro venute dalla pessima stagione che deve averli accompagnati con una rabbia e con una prepotenza non punto meritata da que' nostri eccellenti amici.

Con l'arrivo del Re forse capiterò a Firenze e siccome in quei giorni ella sarà di certo in città, così ho speranza di rivederla ${ }^{3}$.

Riverisca il Sig. Ubaldino, mi rammenti con affetto a tutti gli amici e sono sempre il suo devoto amico

R. Fucini

XCVIII

R. Fucini a E. PeruZZi

Gentilissima Sig. Emilia

Pistoia 12 Aprile 1879

Le mando un saluto rispettoso e mille felici auguri deplorando le vigliaccherie di cui è stato vittima in questo ultimi tempi il Sig. Ubaldino ${ }^{1}$.

2. Fucini fa riferimento ai debiti contratti dal Comune fiorentino negli anni della capitale. Dopo il trasferimento a Roma, infatti, il Governo italiano tolse il proprio appoggio finanziario alla città toscana.

3. Il re giunse a Firenze il 7 novembre 1878 e ripartì il 12 dello stesso mese. Si veda «La Nazione» del 7 e del 11 novembre 1878.

XCVIII. Dall'aut. in BNCF (CEP, cass. 74, ins. 12, n. 11). Bigl. vis. intestato "Renato FuCinI". Busta: «Nobile Sig.ra / Sig.ra Emilia Peruzzi / Antella». Ann. aut. della Peruzzi, a lapis: «Fucini». Inedito.

1. Si tratterà probabilmente delle aggressioni subite dal Peruzzi, considerato da molti responsabile della crisi finanziaria del Comune di Firenze (si veda M. P. Cuccoli, Emilia Toscanelli Peruzzi, cit., p. 203), al Circolo Filologico e poi il I aprile in piazza S. Firenze da un certo Domenico Giorgi. Cfr. «La Nazione» del 9 novembre 1878 e del 3 aprile 1879. 
Si faccia core e pensi che se in questo mondo v'è troppa canaglia, vi sono anche amici che rimangono, e questi amici ella saluterà da parte del suo devot.mo

R. Fucini

\section{XCIX}

\section{R. Fucini a E. Peruzzi}

Gentil.ma Sig. Emilia

Pistoia 21 Aprile 1879

Quando tutti gli altri indignati le avranno indirizzato parole di devozione e di conforto, io tacqui e non avrei potuto fare diversamente perché i fatti che tanto deploro li seppi soltanto pochi giorni $\mathrm{fa}^{1}$.

A lei sembrerà strana questa notizia; le sembrerà incredibile, si maraviglierà, inarcherà le ciglia, batterà i pugni sulla tavola, griderà alla vergogna, riderà forse, ma il fatto è questo; e il suo orrore arriverà al colmo quando le avrò detto che, tranne la Rassegna Settimanale e la Nuova Antologia ${ }^{2}$, io non vedo mai, meno che per eccezione, altri giornali. E se mi accade, come in questa occasione, di sentir parlare d'avvenimenti d'importanza, vado allora a pescare nei fondi del gabinetto di lettura e allora soltanto mi metto al giono di fatti accaduti un mese, o qualche volta anche due mesi innanzi. Leggo molto, sto moltissimo in casa, e quando esco lo faccio per andarmene in campagna a spasso, o alla caccia o alla pesca. Questa è la vita che meno dalla quale attingo molta salute al corpo, parecchia tranquillità allo spirito e tanta sfacciataggine di credermi sul serio un buon cittadino.

Riguardo a quel che mi dice sullo scritto pel 25 Aprile le dirò che avrei tutte le migliori intenzioni del mondo, ma che per troppe cause mi trovo nella impossibilità di far bene. Per tale effetto occorrerebbe uno scritto brillante, pieno dell' umor più raffinato, traboccante di quel vigore di efficacia che non si ricava altro che da una padronanza assoluta della situazione e di ogni più minuta particolarità degli antecedenti per un lungo periodo di cronaca, delle quali cose appunto io manco assolutamente e senza di che non si può mandar fuori altro che uno sbiadito e gracile aborto.

XCIX. Dall'aut. in BNCF (CEP, cass. 74, ins. 12, n. 11 bis). Lettera, 4 ff. su 2 cc. Inedita.

1. Difficile capire a cosa si riferisca Fucini. Forse allude alle dimissioni rassegnate da Ubaldino Peruzzi nell'aprile del 1878 come risposta all'inadeguatezza degli aiuti finanziari concessi dallo Stato al Comune di Firenze. Cfr. «La Nazione» dell'8 aprile 1878.

2. Sulla «Rassegna Settimanale», rivista fondata nel 1878 da Franchetti e Sonnino, si vedano: M. Moretti, Karl Hillebrand e la "Rassegna Settimanale», in L. Borghese (a cura di), Karl Hillebrand eretico d'Europa. Atti del seminario (1-2 novembre), Firenze, Olschki, 1986, pp. 79-125; P. CARLuCCI, La "Rassegna Settimanale» (1878-1882). Il percorso originale di una rivista militante, in M. Augello, M. Bianchini, M.E.L. Guidi (a cura di), Le riviste di economia in Italia (1700-1900), cit., pp. 443-470. Sulla "Nuova Antologia» si veda la nota 1 alla lettera LXXVII. 
Non ho mai saputo fare un sonetto pel S. Carlo di Boldrino ${ }^{3}$. Un sonetto scherzevole mi sarebbe anche riescito facile. $\mathrm{O}$ in che modo non ho saputo farlo? Perché non sono pieno del soggetto; perché non conosco abbastanza il Boldrino.

Che ingegno inconcludente è il mio! Se sapesse, Sig. ${ }^{a}$ Emilia, che pena mi faccio quando m'esamino e mi penso! M'avevan fatto credere che avessi un po' di criterio e qualche attitudine, ma non ci credo più.

Mi scusi e mi compatisca perché me lo merito, e mi tenga sempre fra io suoi più devoti e rispettosi amici

R. Fucini

C

R. Fucini a E. PERUZZI

Gentil.ma Sig. Emilia

Pistoia 27 Novembre 1879

Gratissimo il suo ricordo anche per la mia buona compagna che la ringrazia e la presenta i suoi omaggi.

Secondo me, per intendere la chiusa delle Campane ella è andata a cercare in regioni troppo elevate ${ }^{1}$. Ho inteso di fare semplicemente un confronto fra gli effetti e la causa; dinanzi alle impressioni profonde che il suono d'una campana notturna produce nell'animo mio, ho visto, e mi son divertito a gettargli addosso una nota di ridicolo, quel gonzo qualunque che sbracciandosi e dimenandosi e saltando e sbuffando ne le suscita. È questo il concetto mio, ma temo di non esser riuscito troppo chiaro perché altri mi hanno chiesto spiegazione.

Ella desidera il mio ritorno! Pensi se vagheggio io il giorno che potessi tornar fiorentino in Firenze e lieto fra lieti compagni sulla terrazza dell'Antella! Speriamo, ma intanto l'anni mia passano a volo ${ }^{2}$, e la miglior parte di me si assidera in questa florida e sonnifera conca dell'Appennino.

3. Carlo Emilio Boldrino, «[...] un pacifico, ma malizioso travet piemontese [...]. Era un tipo di piemontese del vecchio stampo. Sempre compìto, prudente, un po' impenetrabile, uomo di sicura amicizia e di ottimo consiglio, era capo del Ministero della Guerra» (cfr. A. Toscanelli Altoviti Avila, Emilia Toscanelli Peruzzi. Notizie e ricordi, cit., p. 22). Non sappiamo a cosa Fucini si riferisca con l'espressione «S. Carlo di Boldrino». Sulla festa di S. Carlo cfr. nota 6 alla lettera LXXIV.

C. Dall'aut. in BNCF (CEP, cass. 74, ins. 12, n. 12). Lettera, 4 ff. su 2 cc. Parzialmente edita in L.G. SBrocchi, Renato Fucini. L'uomo e l'opera, cit., pp. 57-58.

1. Si tratta del sonetto XI dei Cinquanta nuovi sonetti in vernacolo pisano (cfr. R. FucINI, Cinquanta nuovi sonetti in vernacolo pisani, in ID., Tutti gli scritti, cit., pp. 937-938). "Quando ' $r$ vento la notte, alla lontana, / mi porta, 'nsieme ar chiù dell'Assiòlo, / l'affritto rintocca' d'una 'ampana, / mi vien voglia di piange' e d'esse' solo; // e mi metto a pensa' la vita umana, / e mi par tutta pianto e tutta dolo: / penso a' mi' morti, ar Ceppo, alla Befana, / e all'anni mia ch'enno passati a volo. // E cor capo, accosì, giù tra le mane, / mi rassegno ar destino e a' su' dereti, / e bacere' le fune e le 'ampane. // Però nun so capì, Dio mi perdoni, / come diavolo mai faccino 'preti / a trovare 'r coglione che gliele soni».

2. È un verso del sonetto Le 'ampane. 
Ora sono molto occupato; faccio, come ella saprà, le veci del Manfroni finché durerà la sua assenza e tutti i giorni che la scuola mi lascia libero, corro per questi monti biondo spauracchio dei pallidi maestri elementari e dei loro scaruffati marmocchi ${ }^{3}$. Prendo grandine grossa ed acqua tinta e neve peggio d'un dannato del sesto canto, ma la mia salute è buona, sfido le tempeste e trovo questa occupazione confacentissima al mio temperamento ed alla mia natura. Il General Cammarota ha in me un soldato che a molti la cederà per tattica, a nessuno per valore ${ }^{4}$.

A tempo perduto faccio qualche sonetto, ma più che altro lavoro intorno ai miei bozzetti campagnoli, li mando i già fatti e cercando di farne dei nuovi per pubblicarli quando che sia in un volume ${ }^{5}$. Mi farò canzonare?

Se ella fosse stata in città, sarei già stato molte volte a salutarla; ma come fare a salire all'Antella se sono sempre capitato a Firenze per poche ore?

Avrà però ricevuto spero i miei complimenti dallo Stromboli e da altri buoni amici ${ }^{6}$. E a quelli ne aggiunge tanti altri ora il suo devot.mo

R. Fucini

\title{
CI
}

R. Fucini a E. PERUZZi

Pistoia 31 Decembre 1879

Mi rammento alla Sig. ${ }^{a}$ Emilia e al Sig. Ubaldino augurando loro con piena intenzione pace e salute.

Di loro devot.mo amico

\author{
R. Fucini
}

3. Fucini venne nominato ispettore scolastico il 4 novembre 1879. Cfr. L. G. SBROCCHI, Renato Fucini. L'uomo e l'opera, cit., p. 25. Su Mario Manfroni cfr. Introduzione, p. 16, nota 45.

4. Gaetano Cammarota (Napoli 1828-Roma 1909). Frequentò con Pasquale Villari la scuola di fisica e di filosofia di Luigi Palmieri. Nel 1848 combatté sulle barricate rimanendo ferito; curato di nascosto, si imbarcò su una nave che lo portò a Marsiglia, da dove passò a Torino. Qui acquistò la stima di uomini come Silvio Spaventa e conobbe i principali uomini politici del Piemonte. Dopo aver insegnato scienze naturali, divenne ispettore generale al Ministero dell'istruzione a Torino, poi provveditore a Firenze e a Roma.

5. Fucini riunirà gran parte dei bozzetti scritti in questi anni in Le veglie di Neri. Paesi e figure della campagna toscana, edito da Bemporad nel 1882.

6. Pietro Stromboli fu professore di geografia e statistica alla scuola di scienze sociali di Firenze "Cesare Alfieri” (si veda G. Spadolini, Il "Cesare Alfieri” nella storia d'Italia, Firenze, Le Monnier, 1975, p. 189). Fece parte della Società Colombaria e del Circolo filologico (cfr. V. VANnUCCI, Istituzioni fiorentine, cit., p. 281 e 290).

CI. Dall'aut. in BNCF (CEP, cass. 74, ins.12, n. 13). Lettera, 1 f. su 1 c. Inedita. 
Gentil.ma Sig. Emilia

Pistoia 29 Gennaio 1880

Qualche giorno addietro, scrivendo al Sig. Ubaldino, gli dicevo che presto avrei dovuto seccarlo perché spendesse una sua parola a mio benefizio. Credo che il tempo sia venuto o che almeno s'avvicini; ed eccomi all'arrembaggio.

Fino dal principio dell'anno scolastico sostituisco, come ella sa, il Manfroni nell'ispettorato del Circondario di Pistoia. Sono convinto d'aver fatto fino ad oggi il mio dovere con zelo, e sono sicuro di me per l'avvenire, per cui non mi credo né esigente né sfacciato se, dato il caso che il Manfroni lasci vacante il posto come molti dati fanno supporre, aspiro a sostituirlo stabilmente qui, in questo Circondario.

In tal caso credo efficacissima una raccomandazione, una mezza parola che il Sig. Ubaldino volesse, alla prima occasione, pronunziare al Comm. Buonazia dal quale dipende questo affare.

Ho scritto a lei per risparmiare al Sig. Ubaldino almeno la seccatura di leggere la mia lettera; e lei sarà tanto gentile di parlargliene e di usare la sua dolce influenza perché si prenda maggiormente a core la cosa.

Non si dimentichi che ho famiglia, che i miei mezzi sono molto limitati; che la mia posizione attuale è meschina ed incerta, e che il mio onore come cittadino, come padre e come insegnante è, grazie a Dio, fino ad oggi immacolato. Non mi dia d'orgoglioso se faccio tale stima di me; mi son guardato nell'animo, ho visto che è così, e non mi son peritato a dirlo.

Spero che avrà ricevuto verso Capo d'Anno una mia lettera con un augurio. Era cosa breve ma significante poiché, avendo abolito per conto mio il comodo uso delle carte da visita, ho scritto invece, quest'anno, un biglietto per ricordarmi agli amici che maggiormente amo e stimo.

Si tratterranno punto in Firenze quest'inverno? Ha veduto nella Rassegna il mio bozzetto - Nonno in Maremma - che glie n'è parso'? ${ }^{1}$ Son gingilli che faccio volentieri perché mi diverto tanto a farli.

Gradisca i complimenti di mia moglie ${ }^{2}$, ringrazi anticipatamente il Sig. Ubaldino e creda sempre alla devota amicizia del suo obbligatissimo

R. Fucini

CII. Dall'aut. in BNCF (CEP, cass. 74, ins. 12, n. 14). Lettera, 3 ff. su 2 cc. Inedita.

1. Sulla «Rassegna Settimanale», dove Fucini pubblicò la maggior parte dei bozzetti poi raccolti nelle Veglie di Neri, non abbiamo trovato traccia di una novella dal titolo Nonno in Maremma. Potrebbe forse trattarsi di L'oriolo col cuculo, che uscì nella «Rassegna Settimanale» del 6 aprile 1879 (III, 66, pp. 261 264). L’unico altro racconto pubblicato prima del 29 gennaio 1880 è Vanno in Maremma («Rassegna Settimanale», V, 107, 18 gennaio 1880, pp. 48-49).

2. Su Emma Fucini si veda la nota 1 alla lettera II. 


\section{CIII}

\section{E. PeruZzi a R. Fucini}

La sua lettera mi fu un caro ricordo - volevo risponderle subito ma speravo notizie buone, le aspettavo. Ora voglio dirle che Ubaldino è arrivato a Roma, che ha parlato al Ministero, che si è benissimo disposti per lei ma... sempre il ma... ma la sua è legata alla destinazione del Manfroni e fin ora il Mf è in missione e non avrà altro destino finché la missione non sarà compiuta ${ }^{1}$.

Ora prego lei di scrivermi subito chi ha detto che il Manf. era stato destinato in Sicilia? Smentirlo non pare possibile dunque da chi viene? Lo ha saputo e da chi? Al Ministero jeri assicurarono a Ubaldino stesso che per ora bisogna che la missione duri. In consolazione la notizia non è cattiva poiché non è presumibile che compiuta la missione ardua al Mfni non si dia un avanzamento. Allora il posto sarà vuoto e secondo il volere del ${ }^{*}{ }^{*}$ sarà per lei. Tanti saluti a sua moglie. Scriva le novelle come il cuore le detta e spero vederla almeno a Primavera.

Sua Emilia

\section{CIV}

\section{R. Fucini a E. PeruZzi}

\section{Pistoia 6 Febbraio 1880}

Mille e mille grazie, gentilissima e buona signora Emilia, a lei e al sig. Ubaldino al quale dimostrerà il mio core riconoscente. Le notizie non potevano essere migliori perché sono proprio quelle che desideravo.

Badiamo, per carità, che non accadano malintesi che potrebbero essermi dannosissimi. Lo ripeto - Succedere al Manfroni quando il Manfroni per avanzare nella sua carriera avrà lasciato scoperto l'Ispettorato di Pistoia -. Ecco quello che cerco, ed ecco la ragione delle mie premure le quali tendono a preparare il terreno per tempo e niente più. E quando mai ho inteso d'entrare nel posto del Manfroni prima che egli abbia avuto altra e migliore destinazione?

Se al ministero avessero inteso diversamente le mie premure, ne sarei desolato.

Mi giunge affatto nuova la voce del Manfroni destinato in Sicilia, e per me non può esser che una pretta invenzione di qualche maligno o di qualche imbecille. Le notizie che corrono qua sono che egli avrà un avanzamento, ma quando, come e per dove nessuno sa dirlo, ed a nessuno ho sentito rammentare la Sicilia.

CIII. Dall'aut. in BRF (FRF, cass. 8, ins. 19). Lettera intestata "EP”, 4 ff. su 2cc. Anno, (errato), non aut. e a lapis: 1872. Ann. aut. di Fucini (a lapis): «Peruzzi». Inedita.

1. Su Mario Manfroni si veda Introduzione, p. 16, nota 45.

CIV. Dall'aut. in BNCF (CEP, cass. 74, ins. 12, n. 15). Lettera, 3 ff. su 2 cc. Inedita. 
I Bozzetti li scrivo sempre come il core me li detta altrimenti non sarei capace di buttar giù due righe. Mi dispiace che il =Nonno in Maremma= le sia parso dettato da qualche altro viscere; eppure mi è uscito interamente dal cuore ed ispirato tutto dal vero $^{1}$. Glie ne annunzio prossimo un altro festoso e bizzarro che vorrei le piacesse per augurarmi bene della fortuna del volumetto dentro al quale presto li raccoglierò ${ }^{2}$.

Mille complimenti anche da mia moglie la quale unisce i suoi grandi ringraziamenti a quelli del suo devoto amico

R. Fucini

\section{CV}

R. Fucini a U. PeruZZI

Stimatissimo Sig. Ubaldino

Pistoia 12 Novembre 1880

Mille e mille grazie delle consolanti notizie; e Dio voglia che presto un altro passo mi riconduca al dolce consorzio dei miei vecchi e buoni e cari amici fiorentini.

Tanto Lei che la Sig. ${ }^{a}$ Emilia mi credano sempre il loro sincero e rispettoso amico R. Fucini

\section{CVI}

\section{R. Fucini a E. PeruZZI}

Gentil.ma Sig. Emilia

Pistoia 19 Febbraio 1881

A Firenze ancora nò ${ }^{1}$. È presto. Roma non fu fatta in un giorno e le lepri vanno prese col carro.

Al ritorno del Cammarota o alla nomina del successore vedremo se avrò io la fortuna di piacere e d'esser chiamato dal Provv.e come suo aiuto².

1. Si veda la lettera CIII.

2. Si tratta con ogni probabilità di La fatta, pubblicato sulla «Rassegna Settimanale», V (15 febbraio 1880), 111, pp. 128-130.

CV. Dall'aut. in BNCF (CEP, cass. 74, ins. 12, n. 16). Lettera, 1 f. su 1 c. Inedita.

CVI. Dall'aut. in BNCF (CEP, cass. 74, ins. 12, n. 17). Lettera, 4 ff. di 2 cc. Inedita.

1. Così nell'originale.

2. Su Gaetano Cammarota cfr. la nota 4 alla lettera c. 
Ed ora son proprio contento, Sig. Emilia; faccio finalmente un mestiere confacente alla mia natura e, quel che più conta, ho la coscienza tranquillissima perché so quello che faccio.

Gran fatica, però. Non ho libere che le Domeniche, e questa libertà consiste nel potermene stare in casa tutto il giorno e scrivere senza respiro per uffizio e per mie corrispondenze particolari. Verso la metà di Marzo vado a Empoli dove mi tratterrò un paio di mesi avendo avuto l'incarico provvisorio di guardare anche quel Circondario ora che il Gabardi è a supplire da Provveditore ${ }^{3}$.

Venni a Firenze tempo fa, e nel mio programma per que' tre giorni che vi stetti trovavasi in prima linea una desideratissima corsa all'Antella; ma la Sig. Emilia e il Sig. Ubaldino erano a Parigi ${ }^{4}$. E ci mancò un ette che non venissi in ogni modo perché appena in Firenze, mi fu detto soltanto esser partito il Sig. Ubaldino. Ed era già alla diligenza quando mi nacque un dubbio e corsi ad informarmi dal suo Portiere. Avrei voluto scriverle a Parigi, ma non lo feci avendo incaricato il Linaker dei miei più affettuosi ed amichevoli complimenti per loro's

Povero Sig. Ubaldino quanto ha fatto per me! Ma quando m'accosterò a lui si guardi bene perché non garantisco di poter resistere alla tentazione di dargli un abbraccio come lo darei a mio padre. È un po' troppa confidenza, è vero; ma il core è capace di riempire distanze anche maggiori.

Quando capiterò a Firenze? Non lo so. Ma stia pur certa che non vi capiterò senza salire all'Antella dove, più che un sacrosanto dovere, mi chiama il prepotente bisogno di espandermi coi miei migliori e più cari amici.

E questi sentimenti sono quelli della intera mia famiglia che ripete da Loro tutta la contentezza e la soddisfazione che finalmente mi s'è posata sul viso.

A voce gli studj del piano di battaglia per tornare quando che sia a Firenze.

I soliti complimenti del suo devoto amico

R. Fucini

\section{CVII}

\section{R. Fucini a E. PeruZZi}

Gentil.ma Sig. Emilia

Dianella 9 Aprile 1881

3. Gabardo Gabardi Brocchi (Firenze 1845-Rovellasca [Como] 1915). Laureato in giurisprudenza, fu revisore alla Camera e redattore del giornale «Fieramosca». Collaborò con la «Gazzetta d'Italia», la «Gazzetta letteraria» e con i parigini «Figaro» ed «Événement».

4. Si tratta dell'annuale viaggio estivo dei coniugi Peruzzi.

5. Arturo Linaker (Firenze 1856-1932). Frequentò il salotto di Emilia Peruzzi, ove ebbe modo di conoscere il Fucini. Insegnò fino al 1927 nelle scuole medie statali a Trani, a Cagliari e a Firenze, divenendo infine presidente dell'Istituto tecnico Filippo Jacini di Pistoia. Fu dantista emerito, storico e filologo.

CVII. Dall'aut. in BNCF (CEP, cass. 74, ins. 12, n. 18). Bigl. vis. intestato "Renato FucinI". Inedito. 
Di qua da questi colli dove sono a sostituire provvisoriamente l'Ispett. Gabardi che sostituisce il Sig Cammarota che sostituisce il Comm. Gabelli, mando un saluto alla Sig. Emilia e le scrivo non potendole gridare negli orecchi che ai suoi viaggi per tutta l'Europa aggiunga una passeggiata a S. Gimignano dove capitai l'altro giorno per caso e dove trovai maraviglie artistiche non vedute né visibili altrove ${ }^{1}$. È un pezzo importantissimo dell'Italia non conosciuta che vorrei che tutti i miei amici conoscessero per sentirmi far dopo dei ringraziamenti strepitosi.

È una gemma della quale non ha idea senza vederla. Si respira l'aria del medio evo fresca come nel 200; si parla con Dante del più e del meno e si siede familiarmente accanto al Ghirlandaio, al Pinturicchio, a Benozzo Gozzoli, a Puccio Capanna ${ }^{2}$ e a Benedetto da Maiano che dipingono e scolpiscono. Stazione Certaldo; vetturino Gamberucci; Cicerone proposto [?] Ugo Nonni coltissimo e gentilissimo prete. Suo devoto

R. Fucini

\section{CVIII}

\section{R. FuCINI a E. PeruZZI}

Gentil.ma Sig. Emilia

Pistoia 6 Maggio 1881

So da Giorgio Roster che Ella mi ha scritto una lettera ${ }^{1}$, che io non ho ricevuto, forse in risposta a quel mio biglietto nel quale Le facevo un saluto e le parlavo di $S$. Gimignano; conosco, o almeno credo di conoscere il contenuto di questa sua lettera non ricevuta e supponendo rispondo.

Il Cav. Bolasco è provveditore di Firenze; il Cav. Bolasco è da Lei conosciuto; il Cav. Bolasco è quello che può aiutarmi efficacemente a tornare a Firenze; Ella è disposta a parlargli in mio favore. Fin qui va tutto bene.

Ora resterebbe a sapere se il Gabardi fa la caccia al posto di Segretario del Provveditore: e di questo io non so nulla e non ho voluto mai saper nulla per un sentimento di delicatezza verso il Gabardi. Attendo i fatti compiuti per muovermi.

Mi restano, però, due punti interrogativi nella testa: il Gabardi aspirerà veramente a quel posto? Il Cav. Bolasco accetterà il Gabardi? E accettandolo ne sarà contento?

Se il Gabardi aspira a quel posto, lasciamo correre; e lasciamo correre anche se il Bolasco l'accetterà; ma non sarebbe male tendere qualche rete onestissima per il caso che il Provveditore accettasse il Gabardi e poi non ne fosse contento, o per l'altro caso che il

1. Su Gaetano Cammarota si veda la nota 4 alla lettera C.

2. Puccio Capanna, pittore attivo ad Assisi nella prima metà del XIV secolo.

CVIII. Dall'aut. in BNCF (CEP, cass. 74, ins. 12, n. 19). Lettera, 4 ff. su 2 cc. Inedita.

1. Su Giorgio Roster cfr. la nota 2 alla lettera XIV. 
Gabardi possa andar provveditore in qualche provincia come i suoi anni di servizio gli darebbero diritto e il suo servizio straordinario dopo la partenza del buon Cammarota. In ambedue i casi, quando non potessi dispiacere al Cav. Bolasco, le mie aspirazioni sarebbero queste: diventare segretario del Provveditore cambiando il Circondario di Pistoia con quello di S. Miniato che mi sarebbe più comodo avendo io in quelle parti quelle po' di zolle che la Provvidenza mi ha regalato per patrimonio.

Non so se sia riuscito chiaro secondo la mia intenzione; ma conto sul suo intuito e sulla sua buona volontà a vantaggio di questo suo piccolissimo amico che è grato ai Sig.ri Peruzzi di tutte le fortune che gli son toccate e di quelle che gli toccheranno. Suo devoto

R. Fucini

\section{CIX}

\section{R. Fucini a E. Peruzzi}

Gentilissima Sig. ${ }^{a}$ Emilia

Pistoia 19 Giugno 1881

Confidando che non le sia sgradito un piccolo ricordo d'uno de' suoi più devoti amici, mi prendo la libertà di mandarle il mio ritratto. Molto più volentieri sarei venuto in persona; e allora avrei potuto meglio farle osservare come due anni di vita ispettorale, se son capaci di rovinare lo spirito d'un artista, hanno però tali virtù igieniche sul corpo da sostituire al pallido volto d'un cittadino il ceffo abbronzato d'un bel mercante di paglia da cappelli.

Mi scusi, Sig. ${ }^{a}$ Emilia, se non vengo a farle una visita come sarebbe mio sacrosanto dovere perché gli impegni del mio ufficio me ne rubano il tempo.

Lunedì vado in montagna dove mi tratterrò una quindicina di giorni facendo anche una breve sosta alla Lima in quell'alpestre oasi ospitato dai gentilissimi Sig.i Farina ${ }^{1}$; eppoi mi tocca tornare subito a Empoli per fare a spron battuto la $2^{\text {a }}$ visita a tutte le scuole di quel Circondario, secondo l'impegno che ho preso col Ministero, se la fine dell'anno scolastico non mi sorprenderà per via.

Poveri i miei sonetti! Poveri i miei bozzetti campagnoli! Pazienza. Oh! Ma come sono contento d'aver finalmente trovato una occupazione decorosa, geniale e che mi dà modestamente da vivere. Come lavoro volentieri! Quanto debbo a Lei e al Sig. Ubaldino!

CIX. Dall'aut. in BNCF (CEP, cass. 74, ins. 12, n. 19 bis). Lettera, 4 ff. su 2 cc. Inedita.

1. Si tratta di Emilio Farina e di sua moglie Margherita Cini. Nel 1822 i fratelli Giovanni e Cosimo Cini avevano impiantato una cartiera sulle sponde del fiume Lima (San Marcello Pistoiese), fondandovi attorno una sorta di piccolo villaggio, che comprendeva gli alloggi per i dipendenti, una scuola, una chiesa e un caffe. Emilio Farina condivise per alcuni anni la direzione della ditta insieme con il cognato Giovanni Cosimo Cini. Cfr. N. Farina CinI, La famiglia Cini e la Cartiera della Lima (1807-1943). Notizie e documenti raccolti da Neri Farina Cini in memoria del figlio Paolo, Firenze, Le Monnier, 1947. 
E a Firenze quando potrò tornare? Non voglio pensarvi, voglio contentarmi del mio stato e tenermi cara la tranquillità dell'animo che mi fa tanto bene e mi fa essere tanto più buono verso gli altri e verso di me.

Le annunzio una quarta edizione economica de' miei sonetti con molte aggiunte che vedrà la luce in Pistoia al principio dell'autunno ${ }^{2}$, e pei primi dell'inverno un elegante volume de' bozzetti ${ }^{3}$.

Eppoi chiudo addirittura bottega, rendo i ferri alle Muse e incomincio sul serio a prepararmi a fare il nonno perché la mia bambina maggiore ha già quattordici anni.

Riverisca il Sig. Ubaldino, saluti i nostri buoni amici e mi creda sempre suo devot.mo R. Fucini

\section{CX}

E. PeruzZi a R. Fucini

7 Li 82

Carissimo Fucini avevamo qui il suo libro ${ }^{1}$ ed io la penna in mano per ringraziarla quando ho saputo che la ragazzina da me veduta iere sera con tanto piacere ${ }^{2}$, è malata e lei e sua moglie e la nonna in pena. Speriamo speriamo. Non le dico di più - ella mi legge nel cuore e legge nel cuore di Ubaldino.

Stiamo ansiosamente aspettando nuove. Dio ci consoli tutti!

Aff.

Emilia

CXI

R. Fucini a E. PeruZZi

Gentil.ma Sig. Emilia

Li 20 Luglio 1882

2. Si tratta delle Poesie di Neri Tanfucio con l'aggiunta di 50 nuovi sonetti in vernacolo, Pistoia, Tip. Cino, 1882.

3. Fucini si riferisce alle Veglie di Neri, edite a Firenze da Bemporad nel 1882.

CX. Dall'aut. in BRF (FRF, cass. 8, ins. 21). Lettera, 2 ff. su 2 cc. Mese, non aut. e a lapis: luglio. Ann. aut. di Fucini (a matita rossa): «Peruzzi». Inedita.

1. Si fa riferimento alle Veglie di Neri. Paesi e figure della campagna toscana (Firenze, Bemporad, 1882).

2. Si tratta di una delle due figlie di Fucini, Ida o Rita.

CXI. Dall'aut. in BNCF (CEP, cass. 74, ins. 12, n. 20). Lettera, 3 ff. su 2 cc. Inedita. 
Non faccio che trascriverle il telegramma che il dott. Bargioni mi ha dettato in questo momento per Giorgio Roster ${ }^{1}$ :

Ieri leggierissima esacerbazione 38,8. Stamani 37,7. Eruzione copiosissima migliariforme. Sperasi apirepsia imminente.

E finalmente si respira. Ah! Poveri babbi. Una eternità di consolazioni non mi pagherebbero gli spasimi di questo doloroso periodo.

Appena la mia bambina sarà entrata in piena convalescenza si vedrà comparire questo zotico montanaro a stringerle affettuosamente la mano.

Reverisca e ringrazi anche l'eccellente Sig. Ubaldino e mi creda sempre suo devot. mo amico

R. Fucini

\section{CXII}

R. Fucini a E. PeruzZI

Firenze 23 Novembre 1882

Gentil.ma Sig. Emilia

Sono lieto che sia stato accolto favorevolmente l'invito che m'ero fatto a venire all'Antella; ma Domenica non posso perché Domenica, dopo una settimana d'assenza, sarò a riabbracciare la mia famiglia.

La settimana entrante sarò pure a Firenze, e allora in una sera dei primi due o tre giorni comparirò a salutarla.

Che il Sig. Ubaldino sarebbe stato oggi a Roma mi venne in mente appena impostata la lettera; e la ringrazio della premura che si è data di confermarmelo e di rimandare la mia passeggiata perché se desidero molto di riveder lei, altrettanto desidero d'incontrarmi col Sig. Ubaldino.

Sempre suo devot.mo

R. Fucini

1. Su Giorgio Roster si veda la nota 2 alla lettera XIV. Un dottor Gustavo Bargioni, socio del Comitato per l'educazione civile delle classi operarie, viene nominato in V. VAnNUCCI, Istituzioni fiorentine, cit., pp. 245-246.

CXII. Dall'aut. in BNCF (CEP, cass. 74, ins. 12, n. 21). Lettera, 2 ff. su 1 c. Inedita. 


\section{CXIII}

R. Fucini a E. PeruZzi

Firenze 1 Decembre 1882

Gentil.ma Sig. Emilia

La gita all'Antella la proibiscono i fati e bisognerà per questa volta rinunziarci.

Ieri sera alle 4 fui libero dal Cons. Prov. e, nonostante la pessima stagione, corsi in Borgo dei Greci dove la sua portinaia mi disse che nessuna delle solite diligenze era calata dall'Antella ${ }^{1}$. Mi immaginai che questo dipendesse da una nevata solenne e rinunziai a partire; ma in ogni modo, prima di abbandonarmi, volli tentare un vetturino di Piazza della Signoria il quale rispose alla mia domanda, dopo aver dato un'occhiata al cielo, con suoni così barbari da corrispondere ad una tentata grassazione. Allora rinunziai per l'ultima volta alla gita, e fino alla metà del nuovo mese, quando tornerò per qualche giorno a Firenze, non potrò più farla perché domani torno a Pistoia per ingolfarmi di lì fra i geli della montagna dove mi toccherà passare qualche giorno per affari del mio impiego. Sono dispiacentissimo di tutte le contrarietà e molto grato alle Sue amichevoli premure.

Sempre suo devot.mo

R. Fucini

\section{CXIV}

\section{R. Fucini a E. PeruZzi}

Sig. Emilia

Giovedì potrei esser libero. Dico potrei perché essendo malazzato il Segret. Drumetti forse toccherà a me a sostituirlo nell'Adunanza del Cons. Provinciale Scolastico. Guardi quante combinazioni contrarie! Il consiglio però non mi impedirà di venire se sarà sciolto prima delle 4. Come si rimedia? A cose normali io potrei uscir prima; ma voglio uscire dall'Uff. alle 4. Se Ella ha qualche altro amico che Giovedì venga in su, faremo così: se ci sono ci sono. In caso diverso lasciamo correre e rimandiamo la gita

CXIII. Dall'aut. in BNCF (CEP, cass. 74, ins. 13, n. 1). Lettera intestata "R. ISPETTORE SCOLASTICO", 3 ff. su 2 cc. Inedita.

1. Per andare all'Antella c'era un tram Firenze-Bagno a Ripoli e poi si continuava o con mezzi propri o con carrozzelle (le tariffe erano state personalmente approvate da Emilia Peruzzi). Si veda E. BENUCCI, Introduzione a E. DE AMICIS, Un salotto fiorentino del secolo scorso, cit., p. 54.

CXIV. Dall'aut. in BNCF (CEP, cass. 74, ins. 13, n. 1 bis). Bigl. vis. intestato "RenATO FucINI". Inedito. 
all'altra settimana ché sarò sempre in Firenze. Guardi che noia! Questa gita ${ }^{1}$ mi pareva tanto semplice e va a diventare un affare di stato per la mia grande legatura in mezzo a un monte di statistiche da far piangere dalle risa.

Tante grazie e mille complimenti dal suo devot.mo

R. Fucini

Li 28 Novermbre 1882

\section{CXV}

R. Fucini a E. Peruzzi

Gentil.ma Sa. Emilia

Firenze 2 Decembre 1882

La mia lettera di ieri sera avrà completato il Suo orientamento a proposito della perduta gita. Domani è impossibile perché al treno delle 10,40 parto desideratissimo dalla mia famiglia la quale è prevenuta del mio arrivo. Sono varj giorni che manco da casa e sono impaziente di tornarvi. Spero di tornar presto a Firenze e allora riapriremo le trattative. Mille complimenti dal suo devot.mo

R. Fucini

\section{CXVI}

R. FuCINi a E. PERUZZI

Pistoia 23 Marzo 1883

Gentil.ma Sig. Emilia

Il Preside di questo Liceo Forteguerri, Sig. Cav. Sanesi, da molto tempo desidera per la educazione de' suoi figlioli, di essere traslocato a Firenze ${ }^{1}$. Ora dopo la morte del povero Sig. Carbone pare che il momento opportuno si sia presentato ${ }^{2}$.

1. Cassato «che».

CXV. Dall'aut. in BNCF (EP, cass. 74, ins. 13, n. 2). Cart. post. Ind.: «Nobile Signora Emilia Peruzzi / per l'Antella / Firenze». Timbro post. di partenza: 2-12-82. Data, aut. della Peruzzi: 3 Dec. 82. Inedita.

CXVI. Dall'aut. in BNCF (CEP, cass. 74, ins. 13, n. 3). Lettera, 4 ff. su 2 cc. Inedita.

1. Tommaso Sanesi (Castelfiorentino [Firenze] 1830 - ?). Studiò a Firenze presso gli Scolopi. Dopo essere stato precettore privato a Milano, insegnò latino e greco nel liceo pistoiese "Forteguerri", diventandone poi preside.

2. Domenico Carbone (Carbonara Scivia [Alessandria] 1823 - Firenze 1883). Compose la notissima satira Re Tentenna contro il comportamento ambiguo di Carlo Alberto, la cui ira costrinse il Carbone 
Il Sig. Sanesi gode la più lusinghiera stima presso il Ministero e presso il Ro Provveditore; ma gli è stato fatto intendere che il Governo in questa nomina cerca su tutto una persona che per le sue qualità morali possa dare perfettamente nel genio ai Signori fiorentini.

Saputo questo, siccome io amo e stimo grandemente il Sig. Sanesi, mi sono affrettato a parlarne a Lei, perché con la Sua personale influenza ${ }^{3}$ in Firenze e per mezzo di quella del Sig. Ubaldino costì e a Roma voglia concorrere al buon esito delle premure che il Sig. Sanesi sta già facendo.

I Suoi titoli e come scrittore e come Preside non occorre che io li nomini perché al Ministero egli è tanto conosciuto che basta rammentarlo perché sappiano di chi si tratta. Quanto al dare nel genio alla parte eletta di Firenze io credo che a fatica potrebbe trovarsi la persona più meritevole e più adatta.

La reverisco, la prego de' miei complimenti per il Sig. Ubaldino e mi creda suo devot.mo

R. Fucini

\section{CXVII}

R. Fucini a E. PeruZZi

Pistoia 29 Decembre 1883

Gentil.ma Sig. Emilia,

Gradisca per sé e pel Sig. Ubaldino le mie più cordiali e liete felicitazioni.

E il primo dell'anno sarà in Cittàl? Farò una corsa a Firenze e sarei lietissimo di presentarle a voce i miei omaggi.

Io mi vergogno. Quanto tempo sarà che non mi son fatto rivedere? Chi sa! Lultima volta che salutai i Sig.i Peruzzi verso i quali ho tanti obblighi ero biondo e ora sono canuto!

Ma il cuore non m'invecchia e mi sento sempre per tutti i miei buoni amici e specialmente per Lei il medesimo suo devoti.mo

R. Fucini

a lasciare il capoluogo piemontese per riparare prima a Roma, poi a Firenze. Nel 1859 fu nominato provveditore agli studi; fu poi a Pisa con poteri scolastici straordinari e, in seguito, provveditore di Bologna. Venne richiamato a Firenze come provveditore centrale e, dopo la liberazione di Roma, fu il primo provveditore della città, carica che ricoprì fino al 1874. Il suo ultimo incarico fu quello di preside del liceo fiorentino "Dante" (1880-1883).

3. Cassato «costì».

CXVII. Dall'aut. in BNCF (CEP, cass. 74, ins. 13, n. 4). Lettera, 3 ff. su 2 cc. Inedita.

1.Corregge «Fir». 


\section{CXVIII}

\section{R. Fucini a U. PeruZZi}

Stimati. mo Sig. Ubaldino

Pistoia 15 Febbraio 1884

Questa elezione mi è stata a cuore non solamente come buon patriotta e monarchico sfegatatato; ma anche come ispettore scolastico.

Che i quasi analfabeti siano elettori, tiriamo via ${ }^{1}$; ma eletti, poi, no.

E la buona Sig. ${ }^{a}$ Emilia che fa? Io le scrissi per Capodanno e sono addolorato di non aver veduto nessuna risposta.

La riverisco e mi creda suo devot.mo

R. Fucini

CXIX

R. Fucini a E. PeruZZI

Gentilissima Sig. ${ }^{a}$ Emilia

Dianella 21 Ottobre 1884

Quella di poter votare per uno solo di quelli delle schede in giro è una gran consolazione perché quelle coppie a uso polli di mercato mi fanno pietà ${ }^{1}$. E questo, credo, sarà un bene anche pel nostro Pareto in vantaggio del quale ho già fatto dopo che me ne scrisse il Vimercati, e farò anche di più in una gita che son per fare sull'Appennino ${ }^{2}$.

Come è povero il nostro Paese se per mandare avanti un galantuomo occorre legargli alla vita un figuro o una nullità! Che scapperà fuori da questa confusione d'idee? Il caso ce la mandi buona.

CXVIII. Dall'aut. in BNCF (CEP, cass. 74, ins. 13, n. 5). Lettera, 2 ff. su 1 c. Inedita.

1. In base alla riforma elettorale del 1882, si veniva ammessi all'esercizio del suffragio non più per criteri di censo, ma se si sapeva leggere e scrivere o se si pagava una certa somma d'imposta diretta. Cfr. A. CASATI, Sonnino e il problema della riforma elettorale 1870-1912, in Riforme elettorali e democrazia nell'Italia liberale, a cura di Z. Ciuffoletti, Firenze, Centro editoriale toscano, 1988, p. 70.

CXIX. Dall'aut. in BNCF (CEP, cass. 74, ins. 13, n. 6). Lettera, 3 ff. su 2 cc. Inedita.

1. Sotto «quella», «quelli» e «quelle» Fucini ha segnato, rispettivamente, «1», «2» e «3».

2. Pareto (su di lui si veda la nota 2 alla lettera XCII) si candidò nel 1880 nel collegio di Montevarchi e nel 1882 in quello di Pistoia-Prato-San Marcello Pistoiese, riuscendo sconfitto in entrambe le occasioni, mentre nel 1886 rifiutò la proposta fattagli da Ubaldino Peruzzi di una nuova candidatura. Tuttavia, 
Restituisco i saluti di mia moglie e quelli della figliolina recuperata ambedue gratissime alle premure di Lei e del Sig. Ubaldino.

Sempre suo devot.mo amico

R. Fucini

\section{CXX}

R. Fucini a U. Peruzzi

Pistoia 19 Gennaio 1886

Stimat.mo Sig. Ubaldino,

Grazie del suggerimento gradito, e, più che d'ogni cosa, grazie della buona memoria che con tanta benevolenza conserva di me.

Ma il tempo della riparazione s'avvicina a passi di gigante, grandi avvenimenti stanno maturandosi; a Giugno coglieremo i frutti ${ }^{1}$.

Sempre servo devoto e fedele della Sig. ${ }^{a}$ Emilia e di Lei.

R. Fucini

\section{CXXI}

E. Peruzzi a R. Fucini

21 del 86 sera

Quali sono i grandi che eventi [*] le nostre [*] e aspettiamo un suo biglietto o molto meglio alcuni versi. Il Franchetti ricorda $\left[^{*}\right]$ in un bel Sonetto serio - lei ne faccia uno a dialogo - ma lesto e me lo mandi qui con un biglietto del Sig. Procacci e d'altri ${ }^{1}$.

prima d'ora non risultava che egli avesse partecipato, o che avesse pensato di farlo, anche alla tornata del 1884 (cfr. T. Giacalone-Monaco, Introduzione a V. Pareto, Lettere ai Peruzzi, cit., pp. LXIII-LXIV). Su Vimercati si veda la nota 5 alla lettera LXI.

CXX. Dall'aut. in BNCF (CEP, cass. 74, ins. 13, n. 7). Lettera, 1 f. su 1 c. Inedita.

1. Come risulta dalla lettera CXXII, Fucini era in procinto di tornare a Firenze.

CXXI. Dall'aut. in BRF (FRF, cass. 8, ins. 21). Cart. post. Ind.: «All'Ill. mo Signore / Renato Fucini / Ispettore Scolastico / Pistoia». Timbro post.: 22 GEN 86.

1. Leopoldo Franchetti (Livorno 1847-Roma 1917). Si iscrisse ala facoltà di legge di Pisa, avendo come insegnante Pasquale Villari e come compagno Sidney Sonnino, insieme al quale, alcuni anni dopo fondò la «Rassegna Settimanale». La sua costante attenzione ai problemi delle masse rurali lo portò a farsi sostenitore del suffragio universale e di una politica di espansione coloniale. Su di lui si vedano: U. 
Spedirò tutto Domenica e Lunedì. Il 27 è vicino. Si tenga invitato a desinare all'Antella qualunque Domenica - se avvisa tanto meglio- alle 4 a casa Peruzzi Borgo de' Greci suole esserci un legno carico d'amici. Risponda presto e in rima.

E. P.

\section{CXXII}

R. FuCINI a E. PeruZZI

Pistoia 23 Gennaio ‘86

Gentil.ma Sig. Emilia,

Eccomi a spiegare l'arcano. Agli ultimi di Maggio o, alla più lunga, ai primi di Giugno, avrò preso nuovamente domicilio stabile in Firenze. Il nostro Provveditore ha voluto gli Ispettori al centro della provincia, e io da sottoposto fedele e subordinato piego il capo e lascio il mio quieto ritiro di Pistoia.

E allora approfitterò del cortese invito della Sig. Emilia, e allora il figliol prodigo tornerà a battere alle porte amiche, e allora sarà fatta larga, solenne, inalterabile ammenda ai falli commessi. E tutti mi dovranno volere lo stesso bene perché ho sempre voluto il medesimo bene a tutti. E così sia.

A Torino ho già mandato direttamente per mezzo d'un mio caro amico di laggiù, il pittore Calderini ${ }^{1}$.

Prima di chiudere questa lettera passo dal Procacci. Se Ella non troverà niente qui dentro sarà segno che gli non avrà potuto darmi niente.

Zanotti-Bianco, Saggio storico sulla vita e attività politica di Leopoldo Franchetti, Roma, Associazione nazionale per gli interessi del Mezzogiorno d'Italia, 1950; Z. Ciuffoletti, Nota storica a L. FranchetTI, S. Sonnino, Inchiesta in Sicilia, Firenze, Vallecchi, 1974, pp. 281-343; A. JANnAZZO, Introduzione a L. Franchetti, Politica e mafia in Sicilia. Gli inediti del 1876, Napoli, Bibliopolis, 1995, pp. 9-25. Potrebbe tuttavia trattarsi di Augusto Franchetti (Firenze 1840-1905), anch'egli frequentatore del salotto di Emilia. Conseguito il baccalaureato in lettere a Marsiglia nel 1858, tornò in Toscana, dove frequentò la facoltà di giurisprudenza prima a Siena e poi a Pisa, laureandosi nel 1862. Collaborò con «La Nazione» e con la «Nuova Antologia», partecipando contemporaneamente alla vita amministrativa di Firenze. Insegnò diritto costituzionale e storia moderna alla Scuola di scienze sociali "Cesare Alfieri", nata nel 1874. Giovanni Procacci (Pistoia 1836-1887). Nel 1854 si iscrisse alla facoltà di giurisprudenza a Siena. Agli anni universitari risalgono i primi scritti poetici, di argomento patriottico. Laureatosi nel 1858, venne colpito da una grave malattia agli occhi. Nel 1866 fu incaricato dell'insegnamento di filosofia al liceo Forteguerri di Pistoia; l'anno successivo venne nominato professore titolare alla cattedra di letteratura italiana. In seguito ottenne la direzione delle scuole elementari, ginnasiali e tecniche della città toscana.

CXXII. Dall'aut. in BNCF (CEP, cass. 74, ins. 13, n. 8). Lettera, 4 ff. su 2 cc. Inedita.

1. Marco Calderini (Torino 1850-1941). Dopo gli studi classici, s'iscrisse alla Facoltà di lettere dell'Università di Torino. Fu spinto alla pittura da alcuni compagni di studio che frequentavano l'Accademia Albertina, alla quale anche il Calderini decise di iscriversi (1867-1873). Aprì uno studio con un amico, decidendo di dedicarsi completamente all'arte. Fu anche scrittore e critico d'arte, collaborando con diversi giornali («Rassegna Nazionale», «Gazzetta piemontese», «Gazette des Beaux Arts»). 
Dio! Quando ci rivedremo che lunga storia di vicende. E quante esclamazioni della buona Sig. ${ }^{a}$ Emilia allorché rivedrà canuto il già biondo menestrello e ora imminente nonno e Suo sempre devoto amico

R. Fucini!

\section{CXXIII}

E. PeruZZi a R. Fucini

1 Maggio 86

Salutiamo con gioja il suo felicissimo ritorno cari.mo amico e le saremo molto grati se verrà la Domenica 9 Maggio all'Antella trovandosi alle 4 a casa nostra. Che bella cosa che dolce cosa il rivederla de' nostri!

Allegria allegria!

\section{CXXIV}

R. Fucini a E. Peruzzi

\section{Pistoia 5 Maggio 1886}

Grazie infinite, mia buona Sig. Emilia, dei suoi entusiasmi pel mio ritorno. Ne ho bisogno per confortarmi, gli amici di Firenze me lo perdonino, nel dolore di dire addio per sempre a questo idillio di vita che durava da otto anni.

Ma domenica non potrò salire all'Antella perché probabilmente sarò nel fuoco dei preparativi e nella fornace dello sgombero.

La prima domenica di quiete a Firenze, comparirò costassù magari inaspettato ospite. Tanto Le domando e tanto le prometto.

Misericordia! Che lieta confusione di cose avremo da raccontarci. Temo che la prima adunanza non sarà che di preparazione.

Millemila complimenti a Lei e al Sig. Ubaldino dal loro fedele amico

R. Fucini

CXXIII. Dall'aut. in BRF (FRF, cass. 8, ins. 21). Bigl. da vis. intestato "Ubaldino Ed Emilia PeruzZI". Inedito.

CXXIV. Dall'aut. in BNCF (CEP, cass. 74, ins. 13, n. 9). Bigl. da vis. intestato "ReNATO FuCINI". Inedito. 


\section{CXXV}

R. FuCini a E. PERuZzi

Firenze 27 Giugno 1886

Gentil.ma Sig. Emilia

Il mio biglietto tiene luogo di una visita di congedo che con mio grande rincrescimento non ho potuto fare in persona.

Il da fare, dopo le noie terribili di un trasloco, mi ha veramente tormentato quando ${ }^{1}$ avevo più bisogno di riposo. Dopo la bella serata dell'Antella non mi son mai fermato per galoppare la provincia e la Città; e ci ho dovuto incastrare persino una corsa di otto giorni a Roma. E fra tutte ${ }^{2}$ queste belle cose ho avuto compagno indivisibile il pensiero molesto d'una mia figliolina che non sta bene di salute.

Mercoledì porto tutta la famiglia a Livorno dove si tratterranno due mesi, e mi toccherà lasciarla subito perché giovedì devo essere alla Sambuca per gli esami. Di lì avrò da andare a $S$. Marcello e da $S$. Marcello, più in là, tantoché non so nemmeno io quando e dove mi fermerò a prendere un'ora di pace ${ }^{3}$.

Ha sentito di quella povera maestrina che si uccise ${ }^{4}$ ? Era del mio Circondario ed avevo fatto tanto per lei che nell'inchiesta del Ministero mi son trovato corazzato come la Lepanto 5 . Ma, quel che più conta, la mia coscienza mi diceva: non aver rimorsi, ché hai fatto il tuo dovere.

Ella e il Sig. Ubaldino partiranno fra pochi giorni per il consueto viaggio annuale; e così prevedo che fino allo scoprimento della facciata del Duomo non ci potremo rivedere ${ }^{6}$.

Buon viaggio e buon ritorno fra gli amici, dei quali uno fedele è il Suo devot.mo R. Fucini

CXXV. Dall'aut. in BNCF (CEP, cass. 74, ins. 13, n. 10). Lettera, 4 ff. su 2 cc. Inedita.

1.Cancellata una parola illeggibile.

2. Cassato «ho av».

3. Sia San Marcello che Sambuca si trovano nella provincia di Pistoia.

4. Si tratta di Italia Donati, suicidatasi per voci compromettenti circa la sua onestà (cfr. «La Nazione» del 12 giugno 1886). È possibile che questo fatto di cronaca abbia ispirato la novella La maestrina, inserita postuma da Guido Biagi nell'edizione "vociana" delle Veglie di Neri (Firenze, 1921).

5. La Lepanto era una corazzata varata nei cantieri di Livorno nel 1883, progettata da Benedetto Brin.

6. Nel 1886 si svolse l'inaugurazione della facciata di Santa Maria del Fiore, scoperta ufficialmente il 12 maggio 1887 (cfr. G. Spadolini, Firenze fra '800 e '900. Da Porta Pia alletà giolittiana, Firenze, Le Monnier, 1984, p. 89). 


\title{
CXXVI
}

\author{
R. Fucini a E. PeruZzi
}

Gentil.ma Sig. Emilia

Firenze 14 Settembre 1886

Sapevo già del Suo ritorno perché informato da amici comuni ai quali ho spesso chiesto notizie dei Sig. Peruzzi.

Fino a Novembre non avrò libere le domeniche perché consacrate alla mia famiglia in campagna, dalla quale sono stato barbaramente diviso nei due mesi di Luglio e Agosto da essa passati per salute a Livorno mentre io sudavo e gelavo a Firenze. Gli altri giorni della Settimana sono doppiamente occupato in Uffizio ora che il Provveditore è in vacanza.

Grazie del Suo gentile svegliarino che avrò presente alla prima occasione. Mille cose rispettose a Lei e al Sig. Ubaldino dal Suo devot.mo

R. Fucini

\section{CXXVII}

\section{E. PeruzZi a R. Fucini}

Di Villa, 6 Nov. 86

$\mathrm{Ci}$ accorgiamo troppo poco, caro Fucini, che Ella sia tornato a Firenze. Il ministro Genala ha domandato di Lei ${ }^{1}$ : credo che sarà qui domenica ed io Le domando se Lei potrebbe venire quel giorno a desinare all'Antella. La prego di rispondermi se approva e se potrà partire col tram delle 4 dalla Piazza de' Giudici per il Bagno a Ripoli². Mille amichevoli saluti.

P.S. La prego di passare domenica verso le 3 a casa nostra per sapere il modo di venire in $\mathrm{su}^{3}$.

CXXVI. Dall'aut. in BNCF (CEP, cass. 74, ins. 13, n. 11). Lettera, 1 f. su 1 c. Inedita.

CXXVII. Dall'aut. in BRF (FRF, cass. 8, ins. 21). Bigl. vis. intestato "EMilia PeruZzI". Inedito.

1. Francesco Genala (1843-1893). Nel 1862 si laureò in giurisprudenza presso l'università di Siena, per stabilirsi poi a Firenze, dove esercitò l'avvocatura. Intraprese un viaggio a scopo formativo, visitando la Francia, il Belgio, l'Olanda e fermandosi per circa un anno e mezzo a Berlino e poi sei mesi a Londra. Al suo ritorno, Ubaldino Peruzzi lo accolse nella Società Adamo Smith, nel cui ambito si occupò della questione ferroviaria, che costituì l'oggetto principale della sua attività di governo. Lavorò assiduamente per il ripianamento dell'ingente debito contratto dal Municipio di Firenze per far fronte alle opere pubbliche richieste dal trasferimento della capitale, sostenendo che lo Stato dovesse farsi carico di una responsabilità morale verso la città. Nel 1883 divenne ministro dei Lavori Pubblici (governo Depretis), carica che ricoprì ancora nel 1892 per volere di Giolitti. Si veda R. Perna, Scritto commemorativo di Francesco Genala, deputato di Soresina al Parlamento, ministro dei lavori pubblici (1843-1893), Parma, Tip. La bodoniana, 1972.

2. Si veda la nota 1 alla lettera CXIII.

3. Cassato "preciso quale sarà». 


\section{CXXVIII}

R. FuCini a E. PERuZzi

Firenze 6 Gennaio 1887

Gentil.ma Sig. Emilia,

Era tutto organizzato per venire in compagnia del mio collega Ispett. Leoni a presentare in persona i nostri omaggi alla Sig. ${ }^{a}$ Emilia e al Sig. Ubaldino. Ma il diavolo mise la coda ficcandomi nel letto la vigilia di Capo d'anno e non permettendomi d'alzarmi che oggi.

E noti bene che per fare la campana tutta d'un pezzo, il giorno Solenne in un altro letto era la mia moglie e in altri due le mie figlie con febbre a 39 accompagnata da tossiconi ${ }^{1}$, reumi, laringiti e altri simili passatempi.

Ma anche se fossi fatalista non mi darei pena. L'anno 1886 incominciato da tutta la mia famiglia fra le maggiori allegrezze è stato l'anno più disastroso della mia vita. Spero nell'87 perché peggio non poteva cominciare.

Infiniti auguri a Lei e al Sig. Ubaldino invia di grandissimo cuore il Suo devot.mo R. Fucini

\section{CXXIX}

\section{E. PeruzZi a R. Fucini}

Spero, caro Fucini, che l'anno le darà le consolazioni che non le diede il primo giorno, e che lei sperava, come lo speriamo noi, con lei e per lei, in tutto l'anno di grazia 1889. Spero che quello che è rimasto non sia perduto; e una sua visita all'Antella ci sarà sempre carissima. Vorrei che mi dicesse quando avrà una Domenica da regalarci; giacché quello è il giorno in cui anche Ubaldino si trova qua. E ora un'altra domanda: con molta segretezza le domandiamo informazioni intorno al curato di Pistoia Don Paolo Coppini, del quale si dice un gran bene per la bontà, la condotta e l'intelligenza ${ }^{1}$.

CXXVIII. Dall'aut. in BNCF (CEP, cass. 74, ins. 13, n. 12). Lettera, 3 ff. su 2 cc. Inedita.

1. Su Emma Fucini si veda la nota 1 alla lettera II. Fucini aveva due figlie, Ida e Rita.

CXXIX. Dall'aut. in BRF (FRF, cass. 8, ins. 21). Lettera intestata "EP", 3 ff. su 2 cc. Inedita. Ind.: «Al Nobil Uomo / Ill. Sig. Prof. Renato Fucini / Professore nella Scuola Tecnica di Pistola».

1. Don Paolo Coppini, priore di Ruballa; insieme a don Gaetano Cini, priore di San Giorgio, rimase accanto alla Peruzzi durante tutta la durata della sua malattia. Cfr. M. Manfroni, Poscritto a E. Homberger, M. Manfroni, Il Bamboccio. Novella toscana, Firenze, Civelli, 1902, p. 122. 
Risponda appena può, e mi abbia persona

Aff.

Emilia Peruzzi

\title{
CXXX
}

\author{
R. Fucini a E. Peruzzi
}

Gentil.ma Sig. ${ }^{a}$ Emilia

Firenze 31 Gennaio 1887

Niente so dirle del sacerdote Coppini che io conosco appena di vista e del quale non ho mai sentito parlare. Avrei voluto subito chiederne notizie a Pistoia, ma la condizione della molta segretezza che Ella mi impone mi ha trattenuto dal farlo. Ma sono pronto a servirla con molto piacere se vorrà darmene incarico.

Ella non può immaginare con quanta gioja io verrei spesso all'Antella, ma dovrò impormi il sacrifizio di capitarci a corsa e di rado fino a che non avrò trovato un po' d'equilibrio e un po' di pace alla nuova travagliatissima mia vita in questo periodo.

Ora, per esempio, le domeniche le ho consacrate alla mia famiglia che è tutta in campagna dove la condussi, vedendo questa bella stagione, a restaurarsi dopo le costipazioni e le febbri natalizie. Gli altri giorni sono sempre a giro per la Provincia peggio d'un merciajo ambulante. Giudicandone da quando ero a Pistoia ${ }^{1}$, credo in verità che per poter capitare un po' più spesso e con più pace a Firenze mi converrebbe chiedere il Circondario di Caltanissetta o, per lo meno, quello di Iglesias.

Ora domando a Lei un favore. Se ha occasione di scriver presto al nostro Genala, gli raccomandi, La prego, la faccenda della Stazione di Pistoia. Egli sa tutto, io gli ne ho già scritto, ma una Sua parola varrebbe, ne son sicuro, quanto molte delle mie. E La ringrazio.

Reverisca con molta e sincera stima il Sig. Ubaldino e mi creda Suo devot.mo

R. Fucini

CXXX. Dall'aut. in BNCF (CEP, cass. 74, ins. 13, n. 13). Lettera, 4 ff. su 2 cc. Inedita.

1. Nel 1887 si aprì una disputa fra il Comune di Pistoia, che chiedeva un miglioramento estetico della stazione, e la Società delle Ferrovie Meridionali, proprietaria dell'edificio, decisa a non apportare alcuna modifica. Il contrasto si risolverà nel 1889 con la vittoria del Comune di Pistoia e con la ricostruzione della stazione. Si veda A. GiuntinI, La costruzione della stazione ferroviaria di Pistoia, in "Farestoria», VI, 1987, 1-2, pp. 11-20; G. Beneforti, Appunti e documenti per una storia urbanistica di Pistoia (1840-1940), Pistoia, Tellini, 1979, p. 25. Su Francesco Genala cfr. la nota 1 alla lettera cxxvII. 


\title{
CXXXI
}

\author{
R. Fucini a E. Peruzzi
}

Gentil.ma Sig. Emilia,

Ella è un angelo di bontà. Io sono... io sono il figliol prodigo.

Ma domenica, a costo di passare di sul cadavere dell'Uff. ${ }^{\circ}$ prov.e scolastico, verrò all'Antella.

Suo devot.mo servo ed amico

R. Fucini i

\section{CXXXII}

R. Fucini a E. PeruZZI

Gentil.ma Sig. Emilia

Da questa romita spelonca dove con tutta la famiglia mi trovo bloccato dal ghiaccio e dalla neve fino dal giorno di $\mathrm{Ceppo}^{1}$, mando a Lei e al Sig. Ubaldino i miei più cordiali augurj.

Già da qualche giorno dovevano questi essere stati fatti a voce se la stagione scellerata e i non pochi affari non avessero mandato all'aria il fissato fatto col mio buon collega Ispettore Leoni.

Domani, se le vie saranno praticabili da poter condurre in legno alla Stazione le mie femmine, tornerò a Firenze; e di lì comincerò di nuovo a premeditare una gita all'Antella per confermare a voce i sentimenti di devota amicizia che mi legano ai Ssig. Peruzzi.

Suo devot.mo

R. Fucini

CXXXI. Dall'aut. in BNCF (CEP, cass. 74, ins. 13, n. 14). Lettera, 1f. su 1 c. Inedita.

CXXXII Dall'aut. in BNCF (CEP, cass. 74, ins. 13, n. 15). Lettera, 3 ff. su 2 cc. Inedita.

1. Si veda la nota 2 alla lettera LXIX. 


\section{CXXXIII}

R. Fucini a E. Peruzzi

Firenze 31 Gennaio 1888

Grazie, mia buona Sig. Emilia, del pensiero e del dono gentile. Accetto l'augurio, e se me lo permette, appena il primo nipotino sarà a tiro per il passagallo, lo condurrò meco all'Antella per accompagnarmi quella stupenda ode che ho intenzione di scrivere all'Amicizia $^{1}$.

Quanto mi è cara la Sua benevolenza e quella del Sig. Ubaldino!

Suo devotissimo

$$
\text { R. Fucini }
$$

\section{CXXXIV}

\section{R. Fucini a E. PeruZzi}

Gentilissima Sig. Emilia

Firenze 30 Ottobre 1888

Con mio rincrescimento debbo per quest'anno rinunziare al piacere di far parte della lieta comitiva dei Carli ${ }^{1}$.

Non ho un momento di pace. Il cambiamento di Circondario, le operazioni occorrenti per l'attuazione dei nuovi programmi per le scuole mi tengono in giro continuamente quando non debba stare come un disperato.

Domani inizio un giro per tener conferenze in ogni capoluogo di comune e forse non mi basterà tutto il novembre per finirlo.

Auguro ai Carli riuniti un cantore meno indegno di me; non sarà difficile trovarlo. A Lei e al Sig. Ubaldino auguro salute e felicità, mentre ammiro maravigliato la loro benevolenza per questo umile e sincero amico

R. Fucini

CXXXIII. Dall'aut. in BNCF (CEP, cass. 74, ins. 13, n. 16). Lettera, 1 f. su 1 c. Inedita.

1. «Chiama passagallo il popolo toscano quella musica, fatta con uno strumento qualunque - violino, chitarra o mandolino - che serve a riempire i brevi riposi, fra un distico e l'altro, di chi improvvisa ottave o rispetti.» Cfr. R. FuCINI, Le Veglie di Neri, cit., p. 110.

CXXXIV. Dall'aut. in BNCF (CEP, cass. 74, ins. 134, n. 17). Lettera, 2 ff. su 1 c. Inedita.

1. Sulla festa dei Carli si veda la nota 6 alla lettera LXXIV. 


\section{CXXXV}

\section{E. PeruzZi a R. Fucini}

Di Villa 2/11/89

Ho saputo la sua intenzione di venire all'Antella, ma in conclusione non ci vediamo da gran tempo, e mi duole che sia un ritorno a Firenze di cui si gode pochissimo. Desideriamo di averla qui domenica prossima 6 novembre, e La prego di rispondermi sollecitamente, perché vi sono delle disposizioni da prevedere che variano secondo il No degli invitati. Il Genala e il Boldrino che sono qui stasera avranno un gran piacere di vederla domenica ${ }^{1}$. U.no Le dice mille cose: faccia un'invocazione alle muse perché Le ispirino pensieri affettuosi per gli amici Boldrini, Peri e Fontanelli, tre Carli che si festeggeranno domenica ${ }^{2}$. E La saluto amichevolmente

Emilia.

\section{CXXXVI}

\section{R. Fucini a E. PeruZzi}

Empoli $1^{\circ}$ Decembre 1889

Gentil.ma Sig. ${ }^{\text {a Emilia, }}$

Un mio amico, un certo Napoleone Fondi ${ }^{1}$ ingegnere di strade ferrate a Tolentino vorrebbe, non so se per urgenti interessi di famiglia o per salute, esser mandato alla nuova costruzione della strada ferrata Lucca-Aulla ${ }^{2}$.

Egli mi dice che una sola parola del Com. Peruzzi al Capo del personale Com. Meana basterebbe a fargli ottenere quello che desidera.

CXXXV. Dall'aut. in BRF (FRF, cass. 8, ins. 21). Lettera intestata "EP", 3 ff. su 2 cc. Inedita.

1. Su Genala si veda la nota 1 alla lettera CXXVII. Su Boldrini cfr. la nota 3 alla lettera XCIX.

2. Carlo Boldrini potrebbe essere il Boldrino precedentemente citato nella medesima lettera. Carlo Peri era il direttore della Pia Casa del Lavoro (vedi G. Gozzins, Il segreto dell'elemosina. Poveri e carità legale a Firenze 1800-1870, Firenze, Olschki, 1993, pp. 279-285). Carlo Fontanelli (1843-1890). Conseguì la laurea in giurisprudenza all'Università di Siena. Pubblicò molti articoli sulla «Rassegna Nazionale», "L'Economista», «La Nazione». Tenne la cattedra di Economia e Scienza delle Finanze nell' 'stituto di scienze sociali "Cesare Alfieri” di Firenze, del quale fu anche direttore. Eletto socio ordinario dell'Accademia dei Georgofili nel 1871, fu designato segretario della sezione economia politica. Era dotato di una facile vena poetica, di cui faceva prova in occasioni conviviali, come in occasione della festa dei "quattro Carli" celebrata ogni anno in casa Peruzzi nel giorno di S. Carlo.

CXXXVI. Dall'aut. in BNCF (CEP, cass. 74, ins. 13, n. 18). Lettera, 2 ff. su 1 c. Inedita.

1. Napoleone Fondi, ingegnere. Nel 1895 studiò un progetto di tramvia da Poggio a Caiano Pistoia: Dimostrazione dell'utilità di una tramvia a scartamento ordinario Poggio a Caiano - Pistoia, attraversando la città di Pistoia, in continuazione di quella Firenze - Poggio a Caiano, Pistoia, Niccolai, 1895.

2. La costruzione della linea ferroviaria Lucca-Aulla, decisa dal governo italiano, rimase a lungo interrotta. Cfr. «La Nazione» del 10, 16 e 26 novembre 1894. 
Non ardisco affrontare direttamente il Sig. Ubaldino per questo favore. Ella vorrà pregarlo, ed io ringrazierò tutti e due chiedendo scusa della mia sfrontatezza e della seccatura che il sentimento dell'amicizia mi induce a dar Loro.

Che peccato che i Ssig. Peruzzi non debbano aver mai bisogno di me! Vedrebbero allora alla prova quanta sia l'amicizia del Loro devot.mo

R. Fucini

\section{CXXXVII}

\section{E. PeruZZi a R. Fucini}

Antella 14 Dic. 89

Le sue lettere, caro Fucini, sono un vero gioiello; ci sento lei come se lo avessi qui e mi parlasse - tenga come cosa sicura che se non rispondo possono esservi mille ragioni, ma che io le tenga il broncio non è cosa possibile. Anche Ubaldino le vuole un gran bene, e di lei spesso ci avviene di parlare con gli amici rimpiangendo la sua lontananza. Ma che non viene mai a Firenze? Quando sorriderà la primavera le chiederò di venire una domenica da Empoli all'Antella. Ma sa che il Giorgini è a Firenze $^{1}$ ? Lo ha mai conosciuto?

Voglio procurare a sua figlia e a suo genero qualche conoscenza a Rossano ${ }^{2}$. Scrisse Vittoria al Senatore Morelli e non rispose, scrissi io e non risponde ${ }^{3}$. Fò delle ricerche per sapere dove si trova. E che cosa dicono di Rossano quei suoi figliuoli? La civiltà ha pensato che il viaggio era lungo, e non vi è per anco arrivata. Ma sa che io mando ad alcuni amici all'Estero, come strenna, le sua Novelle illustrate? È un volume delizioso 5 .

E ora vengo alle dolenti note. Ubaldino conosce poco o punto il Sig. Meana. Pensammo che doveva conoscerlo bene il Genala, e gli scrissi a Roma. Egli mi risponde parole piene di affetto per lei, pregandola a dispensarlo dal fare la raccomandazione di cui ella mi scrisse. Non mi dà spiegazioni, ma suppongo che avendo conosciuto il Meana essendo ministro, non creda opportuno di chiedergli nulla come Deputato. La sua raccomandazione era fatta di seconda mano; suppongo dunque che fosse una delle tante che si fanno per non volere o non potere dir di no; ma che non debba starle molto a cuore.

CXXXVII. Dall'aut. in BRF (FRF, cass. 8, ins. 21). Lettera intestata "EP”, 4 ff. su 2 cc. Inedita.

1. Su Giorgini si veda la nota 3 alla lettera LXVIII.

2. Si tratta di una località calabrese, in provincia di Cosenza. Si veda A. GRAdilone, Storia di Rossano, Cosenza, MIT, 1967, in particolare il capitolo Gli anni del riscatto e dell'unità, pp. 793-872.

3. Si tratta probabilmente di Donato Morelli (Rogliano [Cosenza] 1824-1902). Patriota e cospiratore, combatté nel 1848 e prese parte attiva a tutti i moti successivi scoppiati nella sua regione. Militò nelle file del partito moderato.

4. Così nell'originale.

5. Non sappiamo a quale edizione delle Veglie di Neri faccia riferimento donna Emilia, dato che la prima illustrata è quella Hoepli del 1890 (cfr. L. G. SBROCCHI, Renato Fucini. L'uomo e l'opera, cit., p. 256). 
Che il Pareto è fidanzato lo sa ${ }^{6}$ ? Sposa la Sig.na Bakunine, nipote della Marchesa Incontri, graziosa e simpatica ${ }^{7}$.

Ci ricordi alla cara sua Moglie e pensi sempre a noi come ad amici fedelissimi Emilia

\title{
CXXXVIII
}

\author{
R. Fucini a E. PeruzZi
}

Empoli 29 Decembre 1889

Gentilissima Sig. Emilia,

Se queste due pagine di foglio diventassero due ali, sarebbe proprio quello che ora mi ci vorrebbe per contentare il mio desiderio; e volerei subito all'Antella a dirle quanto mi è stata cara la sua lettera e a farmi leggere negli occhi invece che sulla carta tutta la mia gratitudine per il bene che mi dimostra. (Mi sono persuaso che un po' di $600 \mathrm{fa}$ bene alla salute anche il terzultimo giorno del 1889).

Mi scusi se non mi faccio vedere all'Antella perché, in verità, mi manca il tempo. Ho tanto da girare per dovere d'uffizio che ho dovuto affatto abolire le girate per diporto. Quella sinecura che era l'impiego d'Ispettore sui primi tempi è diventata ora una faccenda piuttosto grave per l'aumento delle scuole e per la complicanza sempre maggiore della macchina burocratica. Agli affari d'Uffizio aggiunga quelli privati e l'amministrazione del mio piccolo patrimonio, e giudicherà facilmente, Lei che sta sulla breccia, quanto sia vero quello che dico.

Grazie di tutte le loro premure per il mio raccomandato e grazie anche maggiori delle loro buone intenzioni verso la mia figliolina di Rossano. Non si dia pena di presentazioni e raccomandazioni ché ne hanno anche troppe. Quello che occorrerà presto per quei ragazzi sarà di farli rientrare in paesi meno bestiali perché, da quello che mi scrivono, si trovano in mezzo a selvaggi fatti anche peggiori dalle loro pretensioni di gente civile. E quelli sono nostri fratelli! Me lo perdoni il ministro Miceli ${ }^{1}$, ma quelli, per ora, non li chiamerei né anche nostri pigionali.

6. Su Vilfredo Pareto si veda la nota 2 alla lettera XCII.

7. Alexandra (Dina) Bakounine, figlia di Modesto Nicolajevich, residente a Venezia, e di Anna Nicolajevna Wassiltchikov, residente a Mosca. La marchesa Maria Incontri aprì un salotto, punto di raccolta di artisti e politici, sul finire dell'Ottocento (cfr. A. Nesti, Vita di palazzo. Vita quotidiana, riti e passioni nell'aristocrazia fiorentina tra Otto e Novecento, cit., p. 154).

CXXXVIII. Dall'aut. in BNCF (CEP, cass. 74, ins. 13, n. 19). Lettera, 4 ff. su 2 cc. Inedita.

1. Luigi Alfonso Miceli (Fiumefreddo [Cosenza] 1824 - Roma 1906). Patriota, iscritto alla Giovane Italia, partecipò ai moti del 1848 in Calabria, e qui fu segretario del comitato insurrezionale. Costretto a emigrare a Corfù, tornò nel 1849 a difendere la repubblica romana. Nuovamente emigrato e condannato in contumacia, partecipò alla spedizione dei Mille, combattendo a Calatafimi e a Palermo. Dal 1879 al 1881 e dal 1888 al 1891 fu ministro dell'Agricoltura, dell'Industria e del Commercio. 
Ho messo tutti i miei cani al bosco per veder d'ottenere due cose a vantaggio del mio genero-cugino: la sua nomina effettiva perché ora è incaricato, e il suo trasloco. Spero bene perché al Ministero sono contenti del suo servizio. Dio lo voglia. L'avere una figlia adorata tanto lontana e, quel che è peggio, in paesi tanto barbari, è per me un tormento troppo doloroso e una pena così acuta che ho bisogno di liberarmene a costo di qualunque sacrifizio.

Mi rammenti con affetto a tutta la brigata geniale di frequentatori dell'Antella; dica al Sig. Giorgini quanto è grata la memoria che serbo di lui, si rallegri col Pareto e mi saluti il Fontanelli al quale auguro di cuore l'invenzione della vera pomata rigeneratrice del bulbo ${ }^{2}$. A Lei e al Sig. Ubaldino augura ogni bene il suo devotissimo amico

R. Fucini

\section{CXXXIX}

R. Fucini a E. PeruZZi

Gentil.ma Sig. Emilia

Empoli 3 Gennaio 1891

Sebbene io sia capace di stare anche un anno senza rivolgermi con un saluto alle persone che mi sono più care, non sono capace, per grazia di Dio, di dimenticare. Volano gli anni, vedo tutto passare e trasformarsi intorno a me, ma non passa né si trasforma la dolcezza dei ricordi gentili e l'amore verso chi mi ha fatto del bene.

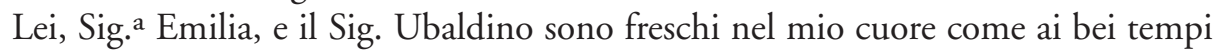
delle serate di Borgo dei Greci, quantunque il biondo e vispo menestrello di Pisa sia diventato il canuto nonno di Empoli.

Sento dire che sono divenuto un uomo serio (forse perché non rido quasi più); ma un tale giudizio mi fa raccapricciare perché, per me, serio, nel senso artistico, non vuol dire altro che rimminchionito.

Ma che ci si fa? Adattiamoci e consoliamoci a malincuore con l'egoistico: - mal comune mezzo gaudio - .

Vivo molto ritirato perché il consorzio del quasinatio borgo selvaggio non fa per me ${ }^{1}$, leggo parecchio e faccio molto l'Ispettore, intercalando raramente tra una lunga noia e un'altra qualche cacciata per queste campagne. La Musa è morta d'asfissia. Dio le perdoni le peccata.

Mi rallegro per la nomina del Sig. Ubaldino a Senatore e sono contento d'aver buone notizie della loro salute ${ }^{2}$. Voglia il cielo che il loro benessere sia costante e si prolunghi quanto lo desidera il Suo devot.mo

R. Fucini

2. Su Carlo Fontanelli cfr. la nota 2 alla lettera CXXXv.

CXXXIX. Dall'aut. in BNCF (CEP, cass. 74, ins. 13, n. 20). Lettera, 4 ff. su 2 cc. Inedita.

1. Si tratterà di Empoli, dove Fucini abitò, salvo alcuni periodi, quando era ispettore scolastico, o di Dianella (cfr. E. Caramelli, Figure di altri tempi, cit., p. 72).

2. Ubaldino Peruzzi era stato nominato senatore il 4 dicembre 1890. 


\title{
CXL
}

\author{
E. PeruZZi a R. FuCini
}

\section{Antella 22 Gennaio 91}

Che nevata, caro Fucini! Non pare vero di stare al canto del fuoco. Lei parla della sua decadenza, ma ne parla con tanta vena, con tanto brio che smentisce quello che dice. Gli anni portano i loro amari frutti: i miei occhi non scrivono più perché non potrebbero dare norma fedele alla penna e le gambe vigorose di Ubaldino chiedono riposo ${ }^{1}$. Chi può vantarsi di non avere la sua croce? Quando il tempo sarà bello l'aspetto un bel giorno, e sarà davvero un giorno bello, a rivedere noi e l'Antella.

I fiorentini domenica dimostrarono che la moderazione regna sempre sovrana nella nostra città ${ }^{2}$. Basta che si muovano!

Ubaldino manda a lei e a tutti i suoi affettuosi auguri ed io vi aggiungo i miei e sono come sempre sua

Peruzzi $^{3}$

Aff.ma

Emilia

\section{CXLI}

E. Peruzzi a R. Fucini

12 Febbraio 1891

Congratulazioni ai nonni ${ }^{1}$.

CXL. Dall'aut. in BRF (FRF, cass. 8, ins. 21). Lettera intestate "EP”, 2 ff. su 2 cc. Ann. aut. e a lapis di Fucini: «Peruzzi». Inedita.

1.Emilia Peruzzi era ormai da anni quasi completamente cieca. Cfr. E. BENUCCI, Introduzione a E. De Amicis, Un salotto fiorentino del secolo scorso, cit., p. 48.

2. Domenica 18 gennaio 1891 a Firenze si tennero le elezioni comunali, con una numerosa affluenza alle urne. Si veda «La Nazione» tra il 18 e il 24 gennaio 1891.

3. Annotazione autografa di Fucini.

CXLI. Dall'aut. in BRF (FRF, cass. 8, ins. 21). Bigl. vis. intestato "Ubaldino Ed Emilia Peruzzi". Data aut. di Fucini e a lapis. Inedito.

1. Si tratterà della nascita di Nerina, figlia di Ida, o di Enzo, figlio di Rita. 


\title{
CXLII
}

\author{
R. Fucini a E. PeruZzi
}

Arrivato a Firenze per venire all'Antella, il Dott. Roster mi dice che gli amici sono pregati a sospendere le loro visite ${ }^{1}$. Dolentissimo lascio questa carta coi miei più caldi augurj.

Firenze 6 Giugno '91

R. Fucini

\section{CXLIII}

\section{E. PeruzZi a R. Fucini}

Antella 7 Giugno 1891

Grazie, caro Fucini, della sua premura amichevole. Ubaldino sta meglio e comincio a sperare in una tregua vittoriosa. Ella avrà spero per mezzo del Roster le notizie nuove ${ }^{1}$. In questa durissima prova mi è stata di sommo conforto la benevolenza degli amici ed Ella tiene tra questi, né abbia l'apprensione della sua gratitudine. Mi saluti la sua signora e le figlie.

8 Giugno. La crisi è superata - cesserà il bollettino. Ieri fu presentata a Ubaldino la stupenda medaglia ${ }^{2}$, preziosa testimonianza d'affetto dei fiorentini al loro Deputato eletto per 30 anni immezzo ${ }^{3}$ a fortunose vicende, raro esempio di fedeltà.

$\mathrm{Ci}$ vedremo consolati spero fra non molto e grazie di cuore

Affe.tta

Emilia Pzi

CXLII. Dall'aut. in BNCF (CEP, cass. 74, ins. 14, n. 1). Bigl vis. intestato "Cav. Prof. Renato Fucini / R. Ispettore SColastico di S. Miniato". Inedito.

1. Il divieto di recarsi all'Antella era dovuto alle cattive condizioni di salute di Ubaldino Peruzzi. Su Roster si veda la nota 2 alla lettera XIV.

CXLIII. Dall'aut. in BRF (FRF, cass. 8, ins. 21). Bigl. vis. listato a lutto. Anno ricavato da riferimento interno («la stupenda medaglia»). Inedito.

1. Su Roster cfr. la nota 2 alla lettera XIV.

2. Nel giugno del 1891 i fiorentini offrirono a Ubaldino Peruzzi una medaglia per ricordare i trent'anni durante i quali egli era stato loro deputato al Parlamento. Cfr. Jarro (G. PICCINI), Vita di Ubaldino Peruzzi, cit., p. 167.

3. Così nell'originale. 


\section{CXLIV}

R. Fucini a U. ed E. Peruzzi

Dianella 27 Luglio 1891

Miei cari Sig. Ubaldino ed Emilia,

Mi arriva un libro che per il titolo - Vita di Ubaldino Peruzzi - e per la dedica - al caro amico Renato Fucini, ricordo affettuoso - mi ha dolcemente commosso ${ }^{1}$. Ma alla dedica non vi è firma. Chi me lo manda? Di certo o il Sig. Ubaldino o la Sig. ${ }^{a}$ Emilia o, piuttosto, tutti e due insieme.

E a tutti e due mando ringraziamenti dal profondo del cuore, mentre mi sento tanto più orgoglioso di quell' - amico - e tanto più intenerito da quel - caro - perché l'animo mio mi assicura che del ghiotto privilegio non sono affatto indegno per la grande stima che ho fatto sempre dei Sig. Peruzzi e per il gran bene che ho sempre loro voluto.

Sono lietissimo che la salute del Sig. Ubaldino, quasi recuperata dal forte amore e dalla forte volontà della Sig. ${ }^{a}$ Emilia, vada sempre megliorando, e faccio voti caldi e continui perché si prolunghi quanto umanamente è possibile la felicità dei miei cari amici dell'Antella.

Di loro devoto e affez.mo

R. Fucini

CXLV

R. Fucini a E. Peruzzi

Mia buona Sig. Emilia,

Empoli 24 Decembre 1891

Per la folla dei conoscenti ho le carte da visita; ai pochi amici, per ricordarmi a loro nelle solenni occasioni, scrivo lettere. Questo ho voluto dirle perché capisca quello che per Lei tiene nel cuore il taciturno di Empoli.

Ma per Lei non ho auguri ${ }^{1}$. Le ho scritto soltanto pensando che una voce amica e i ricordi che questa voce può suscitarle nella memoria, sicuro di qualche conforto alla Sua afflizione.

CXLIV. Dall'aut. in BNCF (CEP, cass. 74, ins. 14, n. 2). Lettera, 4 ff. su 2 cc. Inedita.

1. Si tratta di Jarro (G. PiCCINI), Vita di Ubaldino Peruzzi scritta e pubblicata da lui vivente, Firenze, Paggi, 1891.

CXLV. Dall'aut. in BNCF (CEP, cass. 74, ins. 14, n. 3). Lettera, 3 ff. su 2 cc. Inedita.

1. Emilia era infatti in lutto per il marito Ubaldino, morto il 9 settembre di quell'anno. Si veda M. P. Cuccoli, Emilia Toscanelli Peruzzi, cit., p. 207. 
Come già partecipai a tanta gioia di cotesta casa, partecipo oggi ai suoi dolori. Accolga le espressioni della più sincera amicizia che Le invia il Suo devotissimo

R. Fucini

\section{CXLVI}

\section{E. PeruzZi a R. Fucini}

Firenze - Antella 24 Ott. 92

Chi fu l'ultimo di noi a scrivere? Perché non è venuto a vedermi? Caro Fucini, tutto quello che è avvenuto da quel giorno avevo la forza di vedere gli amici e il Genala mi disse poi quante affettuose parole le suggerì il suo affetto per il mio Ubaldino e per me ${ }^{1}$. Lessi la visita fatta a lei dal Carducci e quando mi se ne offre l'opportunità domando di lei e dei suoi.

Vorrei che mi dicesse il suo giudizio sui versi di Ada Negri e se avesse commesso la colpa di non leggerli, faccia ammenda, li cerchi a Firenze, li legga e me ne scriva ${ }^{2}$. L'introduzione della Sig.ra Albini le dirà che ha 20 anni e scrisse senza aver visto né una gran città, né il mare, né i monti ${ }^{3}$. La sua fibra di poeta deve scuotersi. Ada canta i dolori dell'umanità, ma non dico di più perché desidero che dica lei. Mi ricordi a sua moglie e mi abbia sempre invariabilmente per sua

Aff.ma

Emilia Peruzzi

CXLVI. Dall'aut. in BRF (FRF, cass. 8, ins. 21). Lettera listata a lutto, 2 ff. su 2 cc. Ind.: «Ill.mo Signore / Cav. Renato Fucini / Empoli / per Vinci». Ann. di Fucini sulla busta: «S. Ansano / Nasce un redo [?] angelico / il dì 10 ottobre ' $92 »$. Inedita.

1. Su Francesco Genala si veda la nota 1 alla lettera cxxvII.

2. Si tratta di Fatalità (Milano, Treves, 1892). Sulla scrittrice di Lodi si veda M. PeA, Ada Negri, Milano, Mondatori, 1970; A. Gorini SAnToli, Invito alla lettura di Ada Negri, Milano, Mursia, 1995.

3. Sofia Albini Bisi (Milano 1856 - ?). Figlia di un agronomo e ricco proprietario della Brianza, si sposò con lo scultore Emilio Bisi. Fondò e diresse la «Rivista per le signorine». Fra i suoi scritti si citano Le nozze d'argento, La nidiata e la traduzione del romanzo di Montgomery, Misunderstood. La Peruzzi fa riferimento all'articolo Ada Negri pubblicato sul «Corriere della Sera» del 20-21 dicembre 1891 e riprodotto in numerose edizioni, tra cui anche quella del 1892, di Fatalità. 


\title{
CXLVII
}

\author{
R. Fucini a E. PeruZZi
}

Dianella 5 Novembre 1892

Mia Sig. Emilia,

Chi se ne ricorda, e chi oserebbe farne a Lei carico se per caso fossi stato io l'ultimo a scrivere? Mi ricordo soltanto che dopo la morte del povero Sig. Ubaldino, non avendo potuto condolermi a voce quando venni all'Antella, Le scrissi una lettera nella quale versai tutto il dolore che mi affliggeva e tutto l'affetto che sentivo in cuore per Lei. Non mi ricordo d'altro.

Non sono più tornato all'Antella perché, in verità, il tempo è diventato per me cosa preziosa. Dio buono, questi ministri quante belle cose fanno! Ma quanto aumentano, quanto complicano sempre più anche le minime macchinette burocratiche come sarebbe, fra le altre, quella del mio uffizio. Quando fui impiegato, una dozzina d'anni addietro, mi parve d'essere stato fatto canonico; ora mi par d'essere diventato uno scagnozzo arrapinato, con l'aggravante dello sconforto che mi piglia quando vedo che, gira e rigira, le cose dell'istruzione vanno oggi, dopo tanto lavorio, come andavano press'a poco quando ero canonico gaudente.

Della giovine poetessa Ada Negri non conosco nulla e nemmeno n'ho sentito parlare. Non attribuisca a me una colpa che è tutta della Beozia nella quale il destino mi ha condotto a consumare gli anni della mia virilità matura. Non vedo più nulla, non so più nulla $\mathrm{e}$ quasi non leggo più nulla per al vita che meno e per le persone in mezzo alle quali la meno. Ah mio Dio! Se il compianto Livingstone ${ }^{1}$ si fosse, insieme coi vetturini di Firenze e con l'Av. Barsanti ${ }^{2}$, ricordato anche di me, non parlerei ora a questa maniera. Per grazia del cielo non ho bisogno di nulla, ma se voglio non aver bisogno di nulla, son costretto a far l'impiegato per supplire col mio lavoro a quello che il mio piccolo patrimonio non $\mathrm{mi}$ dà. E così addio passatempi e studi geniali, addio serenità dello spirito, e addio a tutto quello che fa vivere per correr dietro soltanto a tutto quello che fa campare.

Cercherò i versi della Negri, li leggerò e gliene scriverò.

CXLVII. Dall'aut. in BNCF (CEP, cass. 74, ins. 14, n. 4). Lettera, 3 ff. su 1 c. Ind.: «Nobil Donna / Emilia Peruzzi / per l'Antella / Firenze». Ann. sulla busta: «Fucini / Lettera di condoglianze / Il comunicato illusorio / Non sa dei versi di / Ada Negri. Addio / agli studi geniali. / Il Genala. Il Carducci / 6 Nov. 92». Timbro post. di partenza: 5-[?]-92. Timbro post. di arrivo: 16 NOV 92. Inedita.

1. Un Livingstone, americano, viene ricordato per le sue numerose pariglie da U. PESCI, Firenze capitale: 1865-1870. Dagli appunti di un ex cronista, Firenze, Bemporad, 1904, pp. 38 e 363 . Non siamo certi che il Livingstone di cui parla Fucini sia lo stesso da noi rintracciato, né sappiamo a cosa lo scrittore si riferisca nominandolo. Si può ipotizzare che il Livingstone avesse in qualche modo arricchito sia il Barsanti che i vetturini o mentre era ancora in vita o con un lascito testamentario, per cui Fucini si lamenterebbe di non aver beneficiato di questo denaro che gli avrebbe permesso di condurre una vita agiata.

2. Olinto Barsanti (Cascina [Pisa] 1826-Firenze 1905). Liberale, combatté a Curtatone e Montanara. A Firenze fu consigliere comunale e presidente della Congregazione di carità, nonché avvocato di chiara fama. Fu membro di varie commissioni parlamentari e relatore di alcuni progetti di legge. Venne nominato senatore nel 1891 . 
E il nostro bravo e buon Genala ${ }^{3}$ ? Questa volta non c'è l'osso delle Convenzioni, e il nostro amico avrà, ne son sicuro, vita prospera e lunga ${ }^{4}$. Ho il rimorso d'averlo molestato in questi giorni per favorire un mio carissimo amico, ma egli deve aver quello di non aver risposto a una mia ultima lettera. Lo compatisco per i suoi molti e grossi affari, ma una sola riga, anche per rimandarmi in pace, mi sarebbe bastata per mostrare all'amico che io mi ero occupato di lui.

Quanto fui contento della visita del Carducci' ${ }^{5}$ E questa contentezza la rinfrescherò presto perché di giorno in giorno l'aspetto qui a Dianella dove mi ha promesso di venire ${ }^{6}$. Lo voglio portare in pellegrinaggio a Vinci alla casa dove nacque Leonardo, e a Cerreto nella camera dove fu strozzata Isabella Orsini ${ }^{7}$. Chi sa che non ne scappi fuori una nuova ode. E allora i biografi dell'avvenire potranno raccontare che a quella composizione lavorarono insieme col Carducci, il Fucini, il suo modesto cavallo e il suo illustre calesse.

Dai primi vecchi di casa agli ultimi miei tre nipotini, stiamo tutti bene. La mia Emma si unisce con me nell'inviarle complimenti e saluti affettuosissimi.

Suo devoto amico

R. Fucini

3. Su Genala cfr. la lettera CXXvII, nota 1.

4. Si tratta delle Convenzioni ferroviarie, una proposta di legge volta a svincolare le ferrovie italiane dal controllo statale a favore di una gestione privata. Cfr. D. FeLISINI, Ferrovie e finanza: un binomio straordinario, in La rivoluzione dei trasporti in Italia nel XIX secolo. Temi e materiali sullo sviluppo delle ferrovie tra questione nazionale e storia generale, a cura di G. Sabatini, L'Aquila, Amministrazione Provinciale, 1996, pp. 176-177.

5. Riguardo alla visita del Carducci si veda R. FucINI, Acqua passata, cit., p. 638: «Tornando in compagnia di Giosue Carducci da Fucecchio, dove eravamo stati invitati all'inaugurazione della statua a Giuseppe Montanelli, lo pregai di fermarsi a colazione da me, a Empoli. E si fermò». Ermenegarda Caramelli riporta anche la data in cui avvenne questa visita: 17 luglio 1892 (cfr. E. CARAMELli, Figure di altri tempi, cit., pp. 7-8).

6. In realtà Carducci non si recò mai a Dianella. Cfr. R. FucINI, Acqua passata, cit., pp. 640-641: «Da molto tempo il Carducci m'aveva promesso di venire a Dianella dove mi struggevo di condurlo, non tanto per me quanto per mio padre [...]. L'ebbi una volta ospite in casa mia, a Empoli, dove ero ispettore scolastico di quel circondario; ma al mio maniero non potetti averlo mai».

7. Isabella Orsini (Firenze 1542 - Cerreto Guidi [Firenze] 1576). Figlia di Cosimo de’ Medici, venne sospettata di incesto col padre. Nel 1558 sposò Paolo Giordano Orsini, duca di Bracciano, un uomo di guerra celebre più per i suoi delitti che per la sua condotta militare. Egli la accusò di averlo tradito col cugino Troilo Orsino e, la notte del 6 luglio 1576, la strozzò mentre era addormentata nel suo letto. Cerreto Guidi si trova nei pressi di Empoli. 


\section{CXLVIII}

\section{E. Peruzzi a R. Fucini}

\section{Antella 17 Nov. 92}

La sua lettera, caro Fucini, fu per me una consolazione. Mi pareva di sentirla discorrere perché davvero si le stile c'est l'homme, nella sua lettera ci ritrovavo tutto lei. Scrissi al nostro Amico Genala il suo dolce lamento; egli è venuto qui ieri e mi ha detto di salutarla caramente e dirle che appena ebbe la mia lettera esaminò la sua corrispondenza e l'ultima lettera di lei è quella che gli scrisse da Catania ${ }^{1}$. Vede dunque che quando si lamentava non vi era di che, scriva di bel novo per il suo Amico e avrà la risposta che desidera.

Il libro di Ada Negri "Fatalità" a Firenze si trova. Spero dunque che a quest'ora lei avrà letto, riletto e meditato. Non le pare che vi sia grande vigore e molta originalità? La sorpresa cresce pensando che quei versi li scrisse una ragazza di poco più che 20 anni, vissuta a Lodi e relegata poi nella Beozia che si chiama Motta Visconti ${ }^{2}$. Vi sono accenti strazianti, ma quando, come nel Birichino di strada, nelle Nenie materne, nella Pietà, ricorda l'infanzia e sua Madre, si sente un cuore affettuoso che parla ${ }^{3}$.

Che bella cosa se dopo avermi scritto lei ora, potrà più tardi scrivermi il giudizio del Carducci! Mi ricordi con affetto a tutti i suoi e mi abbia sempre per sua

Aff.ma

Emilia Peruzzi

\section{CXLIX}

\section{R. Fucini a E. Peruzzi}

Empoli 19 novembre 1892

Grazie, mia buona, mia eccellente Sig. Emilia. Qualche amico deve avermi fatto il servizio poco amichevole di non mandare al Genala la mia ultima lettera. Ma io perdo-

CXLVIII. Dall'aut. in BRF (FRF, cass. 8, ins. 21). Lettera intestata "EP" e listata a lutto, 3 ff. su 2 cc. Ind.: «Ill. mo Sig.re Fucini / 14 Piazza d'Azeglio». Annotaz. (di Fucini?) sulla busta: «144 [cassato] / 54 Sacca / a £ 12 / 54 12_ 108 / 54/ 648». Inedita.

1. Fucini si trovava a Catania per compiere l'ascesa dell'Etna. Si veda R. Fucini, Su l'Etna, in ID., Foglie al vento, cit., pp. 768-781: "Il giorno di poi, sul far della sera, coi miei compagni di viaggio, prendemmo la via del Monte provvisti abbondantemente di buona volontà, di buon umore e di grossi mantelli siciliani» (p. 770).

Su Francesco Genala si veda la nota 1 alla lettera CXXVII.

2. A Motta Visconti la Negri insegnò per alcuni anni.

3. Si tratta di tre componimenti raccolti in Fatalità.

CXLIX. Dall'aut. in BNCF (CEP, cass. 74, ins. 14, n. 5). Lettera, 4 ff. su 2 cc. Ind.: «Nobil Donna / Emilia Peruzzi / per l'Antella / Firenze». Ann. sulla busta: «Fucinil Ada Negri. Il / suo giudizio favo / revole. G[ena]la / Il venir giù / 20 Nov. 92». Timbro post. di partenza: 19-11-92. Timbro post. d'arrivo: 20 NOV 92. Inedita. 
no sempre e a tutti, specialmente quando il cuore mi gongola dalla contentezza. Non era possibile, e lo pensavo, che il mio Genala m'avesse cancellato dall'albo dei suoi più innamorati amici per mettermi in quello dei conoscenti molesti.

Ho visto il libro della Negri. Anche di questo la ringrazio perché, veramente, il non conoscerlo era per me, sebbene involontariamente colpevole, una vergogna. Bella roba quella! Quanta originalità, quanta robusta malinconia di concetti, quanta nobile eleganza di forma! Ed è una povera maestrina di scuola rurale! ! Ma già ho torto a deplorare. Fra pochi mesi, ne sono sicuro, la Negri sarà direttrice di qualche istituto governativo, e così, a fin di bene, me l'avranno conciata per il dì delle feste stritolandola fra le rotacce sgangherate della macchinaccia burocratica che non perdona neanche a chi ha le membra d'acciaio e l'anima di diamante.

Lei che è tanto buona, perdoni questa zaffata di ribellione a un traviato che, per lontana analogia, Dio gli perdoni le peccata, pochi minuti fa pensava a un neo-eletto deputato analfabeta il quale ha speso, si dice, centomila lire per farsi eleggere, e al quale non è venuto né verrà mai in mente di spenderne una metà per soccorrere una famiglia onorata caduta nella miseria, né per un giovane d'ingegno che non abbaia mezzi di continuare, ne $e^{2}$ per una maestrina Negri la quale, con 50 mila lire e la tranquillità dell'animo, potrebbe forse dare un tesoro alla letteratura del suo paese. La necessità della lotta per giungere alla sommità è una utopia di tempi barbari, che la civiltà moderna dovrebbe scancellare.

Perché costringere un galantuomo a rampicare in cima al Vesuvio con le mani e coi piedi quando c’è la funicolare?

In premio di questi bellissimi ragionamenti, non chiedo a Lei una patente di pensatore; mi basta quella che ho già di buon diavolo e di suo devoto e affettuoso amico.

R. Fucini

P.S. Quando verrà da me il Carducci, Le scriverò subito il suo giudizio sulla Negri; quando verrà il Genala all'Antella, mi scriva, prevenendomi, se sarà possibile, un giorno o due avanti. Chi sa che non potessi bruciare le navi e fare una rapida corsa costassù. Ho accomodato col Genala scrivendogli nuovamente. Si trattava di cosa che mi stava troppo al cuore. Vedendolo o scrivendogli, vuole aiutarmi a muoverlo in favore d'una famiglia di galantuomini che gli ho raccomandato? Grazie.

1. Si veda la nota 2 alla lettera CXLVIII.

2. Così nell'originale. 
Mia gentile Sig. Emilia,

Le memorie d'un Ispettore, ormai non le scriverò ${ }^{1}$. Ho tanto da occuparmi per far l'Ispettore, che è un miracolo se non perdo dietro a lui la memoria d'aver saputo un tempo leggere e scrivere come un uomo. Scriverò piuttosto, in ogni pagina dell'agenda, un pensiero affettuoso per lei. Ma per tale scopo l'agenda mi par piccola, e pochi i giorni dell'anno. Farò quello che sarà possibile, e intanto La ringrazio con tutto il cuore della benevolenza che serba a questo arrugginito cavalier rusticano.

E ora parliamo brevemente del libro del De Marchi, che le restituisco ${ }^{2}$. Ella, secondo me, avendomi sentito raccontare che nell'autunno mi era toccato ingozzarmi 59 volumi d'opere scolastiche, ha voluto fare, con una crudeltà della quale non l'avrei mai ritenuta capace, il numero tondo di 60 . Delle paginacce fatte assai bene come dramma da Arena, ce ne sono; ma nell'insieme è roba da appendice del Secolo, condita con una lingua che è difficile trovarne della peggio in un libro stampato.

Delle agende seguiti pure a mandarmene fine al 1993; ma delle Arabelle, se ha viscere umana, non me ne infligga piu ${ }^{3}$.

Il Genala è stato da Lei? Ha da dirmi nulla del mio raccomandato Ing. Negri?

Ho l'inchiostro gelato nel calamaio, il termometro fuori di finestra segna $7^{\circ}$ sotto zero, e devo andare or ora a Montaione ${ }^{4}$ dove vedo di gran neve e dove mi aspetta un disperato che ha chiesto la patente per titoli. "Bella pagina per le memorie d'un Ispettore!" dice la Sig. a Emilia. "Dio ci assista tutti e due!" dice, augurandole ogni bene, il suo fedele amico

R. Fucini

CL. Dall'aut. in BNCF (CEP, cass. 74, ins. 14, n. 5 bis). Lettera, 4 ff. su 2 cc. Ind.: «Nobil Donna / Sig. Emilia Peruzzi/ per l'Antella / Firenze». Ann. sulla busta: «Fucini / Ringrazia / per l'agenda con / parole aff. se / Ah! Povera / Arabella! che / severo giudizio! / 16 Gen 93». Timbro post. di partenza: 15-193. Timbro post. d'arrivo: 16 GEN 93. Inedita.

1. Sull'intenzione di Fucini di scrivere Le Memorie di un ispettore si veda E. CARAMELLI, Figure di altri tempi, cit., pp. 18-19: «Colpito dalla stranezza dei fatti che gli capitavano durante le visite che faceva alle scuole, fu più volte tentato di raccoglierli in un volumetto che avrebbe voluto intitolare: Memorie di un Ispettore, ma non si risolvé mai, perché troppo lunghi a scriversi e troppo delicati a narrarsi per l'ufficio che egli occupava».

2. Nel 1892 Emilio De Marchi pubblicò a puntate sul «Corriere della Sera» il romanzo Arabella, edito l'anno successivo dall'editore milanese Galli. Sul De Marchi si veda V. BRAnCA, Emilio De Marchi, Brescia, Morcelliana, 1946; D. MatTALIA, Emilio De Marchi, in Letteratura italiana. I minori, Milano, Marzorati, 1962, vol. IV, pp. 3171-3198; A. Gorini SANTOLI, Invito alla lettura di Emilio De Marchi, Milano, Mursia, 1986.

3. Così nell'originale.

4. Località situata tra la Val d'Elsa e la Val d'Evola, in provincia di Firenze. 


\section{CLI}

\section{E. PeruZzi a R. Fucini}

\section{Firenze - Antella 2 Marzo 93}

Se lei, caro amico vuol guadagnare 500 lire faccia una novella come sa farle lei e la mandi avanti il 31 Marzo alla Segreteria dell'Istituto Lombardo a Milano e metta un motto ripetuto in busta suggellata contenente il nome dell'Autore. Ogni novella si aggiri sopra un argomento chiaro e non abbia meno di un foglio di stampa né più di tre. Non abbia il tipo infantile e sia del genere delle novelle di Mérimée, di Heyse, di Turgheneff' ${ }^{1}$ Le sei migliori novelle saranno stampate dalla rivista "Natura ed Arte" illustrate da abili artisti e retribuite dall'editore Cecilio Vallardi ${ }^{2}$. Vi è pure un premio di $£ 300$ per un bozzetto di scene e costumi popolari italiani (affare proprio per lei) e non deve occupare meno di 8 pagine di stampa in $8^{\circ}$ e non più di 16 . S'intende che tanto la novella che il bozzetto destinati alle famiglie debbano avere un indirizzo morale. Si ponga all'opera, faccia un regalo di Pasqua al paese e a se stesso

Sua aff.ma

E. Pzi

\section{CLII}

R. Fucini a E. PERUZZI

Empoli 6 Marzo 1893

CLI. Dall'aut. in BRF (FRF, cass. 8, ins. 21). Cart. post. Ind.: «All'Ill. mo Signore / Cav. Renato Fucini / R. Ispettore Scolastico / Empoli». Inedita.

1. Su Prosper Mérimée (Parigi 1803-Cannes 1870), noto novelliere francese, si veda P. LÉon, Mérimée et son temps, Paris, PUF, 1962; F. Fiorentino, I gendarmi e la macchia. L'esotismo nella narrativa di Mérimée, Padova, 1978; J. AuTin, Prosper Mérimée écrivain, archéologue, homme politique, Paris, Perrin, 1983. Su Paul von Heyse (Berlino 1830-Monaco 1914), autore di più di centocinquanta racconti e premio Nobel per la letteratura nel 1910, si veda R. Bertolozzi, Il mito italiano di Paul Heyse. Studi e documenti, Verona, Fiorini, 1987. Su Ivan Sergeevi Turgenev (Orël 1818-Bougival [Parigi] 1883) si veda V. Sklovskij, Il realismo di Turgenev, in I. Turgenev, Memorie di un cacciatore, Torino, Einaudi, 1964, pp. VII-XXvi; V. GibelLI, Turgheniev, Milano, Giuffré, 1974; J. Bonamour, Turgenev, in Storia della civiltà letteraria russa, Torino, UTET, 1997, vol. I, pp. 654-665.

2. Cecilio Vallardi, editore milanese. Nel 1880 aveva ereditato la casa editrice dal padre Francesco, che ne era stato il fondatore.

CLII. Dall'aut. in BNCF (CEP, cass. 74, ins. 14, n. 6). Lettera, 4 ff. su 2 cc. Ind.: «Nobil Donna / Emilia Peruzzi / per l'Antella / Firenze». Ann. sulla busta: «Fucini / Chiama la / lepre a correre / Il monumento al Chiarugi. Il / suo raccomandato / Negri / 7 Marzo 93». Timbro post. di partenza: 6-3-93. Timbro post. d'arrivo illeggibile. Inedita. 
Ella, mia eccellente Sig. Emilia, invita la lepre a correre ${ }^{1}$; e la lepre ci si proverà sebbene abbia le gambe impigliate in una montagna di desolanti pastoje.

La grandine d'affari continua sul mio tavolino con tendenza marcata, Dio ci liberi tutti, a una certa recrudescenza; e precisamente ora, come se il caso ci si divertisse, mi capitano inviti a scrivere da tutte le parti.

Da Firenze mi chiedono una conferenza per la sala Ginori ${ }^{2}$; da Roma me la chiedono per la Dante Alighieri al Collegio Romano ${ }^{3}$; da Londra mi chiedono scritti per una rivista della quale non m'è riescito nemmeno decifrare il nome.

Il tempo da tutte le parti è stretto e non so a che santo votarmi. Mi brilla l'oro e la gloria davanti agli occhi, e, nuovo Tantalo in soprabito e cilindro, debbo tener le mani nel bozzaccio dei fogli ufficiali invece d'allungarle alla gloria e all'oro ${ }^{4}$.

La ringrazio di vero cuore dello svegliarino a fondo affettuoso, e l'assicuro che farò di tutto per contentarla se da qualche spiraglio mi verrà addosso una corrente di pace.

Per premure fattemi, le mando questa nota di sottoscrizione per un monumento a Vincenzo Chiarugi ${ }^{5}$. Se non le piacesse occuparsene, me la rimandi pari pari (o meglio non me la rimandi scrivendomi) e amici più di prima.

1. Fucini fa riferimento al concorso indetto dall'editore Vallardi, di cui la Peruzzi gli ha dato notizia nella lettera precedente.

2. Tra il 1890 e il 1895 si tennero dei cicli di conferenze nel salone del primo piano del palazzo Ginori (via de' Ginori, 11), ai quali presero parte alcune fra le personalità più in vista di Firenze, come il Bonghi, il Villari e il Carducci. Le conferenze vennero pubblicate dall'editore Treves (La vita italiana, Milano, Treves, 1890-1895). Cfr. L. GinORI LisCI, I palazzi di Firenze nella storia e nell'arte, cit., vol. I, pp. $347-$ 354. Non ci risulta che Fucini abbia mai tenuto una conferenza nella sala Ginori.

3. La Società "Dante Alighieri" si costituì formalmente nel 1889 con lo scopo di operare quale centro di promozione per la tutela e la diffusione della lingua e della cultura italiana nel mondo. Cfr. F. Caparelli, La "Dante Alighieri", Roma, Bonacci, 1987. Il Collegio Romano fu fondato dai Gesuiti nel 1550, per iniziativa di S. Ignazio di Loyola, allo scopo di rigenerare le condizioni culturali e morali della società romana dell'epoca. Nel 1870 l'istituto passò nelle mani del governo italiano, che ne fece una scuola statale (il Ginnasio Liceo "Ennio Quirino Visconti”). Alcuni locali vennero attribuiti a diversi enti. Si veda C. Piersanti, Origini, vicende e glorie del "Collegio Romano" e del Liceo Ginnasio "E. Q. Visconti", Roma, Signorelli, 1958. Un brano di Acqua passata testimonia che Fucini tenne effettivamente una conferenza per la Società Dante Alighieri: «Invitato dalla società Dante Alighieri, andai a Roma per abbaiare una conferenza, non ricordo più in qual sala della città» (cfr. R. FucINI, Acqua passata, cit., p. 629). Non sappiamo, tuttavia, se sia la medesima conferenza cui lo scrittore allude nella nostra lettera, né quale fosse l'argomento trattato.

4. Secondo il mito, Tantalo, avendo offerto come pasto le carni del proprio figlio Pelope agli dei per saggiarne l'onniscienza, dopo la morte sarebbe stato condannato a soffrire in eterno la fame e la sete per il ritirarsi, al suo appressarsi, delle acque e dei rami carichi di frutti da cui era circondato.

5. Vincenzo Chiarugi (Empoli 1759-Firenze 1820). Iscrittosi alla facoltà medica di Pisa e laureatosi appena ventenne, passò all'ospedale fiorentino di Santa Maria Nuova per il periodo di praticantato. Nel 1788 assunse la direzione dell'ospedale Bonifazio, riservato ai malati di mente come già il Santa Dorotea, cui era stato distaccato alcuni ani prima. Fu membro ordinario dei Georgofili, della Colombaria e di altre importanti accademie, e medico della granduchessa di Toscana Elisa Bonaparte. Come membro del Collegio medico, fece parte delle commissioni di studio sulle epidemie febbrili del 1804-1807. Si vedano: E. CotURRI, Le sostanziali innovazioni introdotte in psichiatria da Vincenzo Chiarugi, in «Episteme», VI, 1972, pp. 252-265; P.L. CaBRAS, E. CAMPANINI, D. LipPI, Uno psichiatra prima della psichiatria. Vincenzo Chiarugi ed il trattato Della pazzia in genere, e in specie, 1793-1794, Firenze, Scientific press, 1993. 
Parlò al Genala ${ }^{6}$ del mio raccomandato Ing. Eugenio Negri il quale, fra pochi giorni, dopo il licenziamento in massa degli impiegati straordinarj dello stato e dopo 43 anni di servizio, si troverà in mezzo a una strada con a carico un nipotino e la vedova d'un altro ingegnere che dopo 23 anni morì senza diritto a pensione? Può darmi qualche buona notizia?

Fui, sono e sarò sempre uno dei suoi migliori amici.

R. Fucini

\section{CLIII}

E. PeruzZi a R. Fucini

Bagni di Casciana ${ }^{1}$

$8 / 7 / 93$

Mille congratulazioni².

Mia buona e gentile Sig. Emilia,

Son già passati otto giorni da quando ricevei le sue congratulazioni per la nuova crociata contro di me, e non le ho ancora risposto! Ma veramente la cosa sta così: pensai che costà ossia lassù si sarebbe trattenuta poco, e che la mia lettera si sarebbe esposta a dei lunghi e pericolosi giri, e decisi di aspettare qualche giorno per poterla trovare a colpo sicuro all'Antella. Misi in previsione tre o quattro giorni, e invece sono stati quasi nove.

6. Su Genala cfr. la nota 1 alla lettera CXXVII.

CLIII. Dall'aut. in BRF (FRF, cass. 8, ins. 22). Bigl. vis. intestato "EMILIA PERUZZI" listato a lutto. Ind.: «All'Ill. mo Signore / Cav. Renato Fucini / Empoli per Vinci». Inedito.

1. Località toscana che ebbe origine e celebrità dalle sue acque termali. Si trova nei pressi di Pontedera.

2. Le congratulazioni sono forse dovute al fatto che l'8 giugno 1893 Fucini era stato nominato Cavaliere dell'Ordine dei Santi Maurizio e Lazzaro. Cfr. L. G. SBRoCCHI, Renato Fucini. L'uomo e l'opera, cit., p. 27.

CLIV. Dall'aut. in BNCF (CEP, cass. 74, ins. 14, n. 7). Lettera, 4 ff. su 2 cc. Ind.: «Nobil Donna / Emilia Peruzzi / per l'Antella / Firenze». Ann. sulla busta: «Fucini / Ringrazia per le / mie congratulazioni / Si provò a fare il / bozzetto, ne fu / scontento e lo mise / da parte. Vor / rebbe fare tante / cose e non ne / fa punte / 19 Luglio 93». Timbro post. di partenza: 18.7.93. Timbro post. d'arrivo: 19 LUG 93. Inedita. 
Che tormento è il mio, Signora Emilia; che tortura continua è quella di vergare a mala pena un solo foglio col cuore sopra una risma di carta che son costretto a imbrattare di odiose pedanterie burocratiche!

Dopo il suo invito a concorrerere ${ }^{1}$ a quel racconto per Milano, mille e mille volte, come avrà visto dalle lettere che non ha ricevuto, non le scrissi ${ }^{2}$. Mi avventai con entusiasmo a quell'amichevole suggerimento, mi misi a tavolino sentendomi scoppiettare nel cervello qualche scintilla dell'antico fuoco; ma mi sorprese in viaggio tanta pioggia gelata che le scintille si spensero e il racconto venne alla luce tanto esile e tanto infreddolito che un filantropo, vedendolo, si sarebbe levato il pastrano per buttarlo addosso a lui. Io mi contentai di chiuderlo in una cassetta a dormire placidamente con alcuni suoi fratellini. Questo ho voluto dirle perché, capitandole notizie dell'esito di quel concorso, non mi credesse bocciato.

Ebbi da Dreino Digerini buone notizie della Sua salute e mi compiacqui tanto dell'amore col quale quel caro figliolo mi parlava di $\mathrm{Lei}^{3}$. Fra pochi giorni vado a trovarlo alla Versiliana ${ }^{4}$, come vorrei andare all'Elba, come vorrei venire all'Antella e in cento altri luoghi di dolce ricreazione per il mio spirito arrugginito dalla noia d'Empoli. Ma come debbo fare a contentare con me tanti amici? Accade delle gite quello che accade delle lettere: tutti i giorni vorrei scriverne una, e tutti i giorni non ne scrivo punte perché non posso.

Le chiedo un favore: quando non veda mie lettere, invece di pensar male di me, pensi che pochi dei vecchi amici Le vogliono il bene che Le vuole il Suo devot.mo

R. Fucini

\section{CLV}

E. Peruzzi a R. Fucini

Antella 5 Agosto 93

Lei non vuole scrivere né versi né prose e mise da parte a dormire il bozzetto fatto per il concorso di Milano. Non gliela meno buona e vorrei che almeno nelle vacanze scrivesse le sue ispirazioni. Vide quello che dice di lei il Sig. René Bazin nella

1.Così nell'originale.

2. Si veda la lettera CLI.

3. Andrea Digerini Nuti, socio del Casino Borghesi, fu sindaco di Calenzano (cfr. V. VAnnucci, Istituzioni fiorentine, cit., p. 357).

4. La tenuta della Versiliana, di proprietà dei conti Digerini-Nuti, si trova a Fiumetto, nel comune di Pietrasanta (Lucca).

CLV. Dall'aut. in BRF (FRF, cass. 8, ins. 22). Lettera intestata "EP" e listata a lutto, 4 ff. su 2 cc. Inedita. 
Revue des deux Mondes del primo luglio ${ }^{1}$. Il Bazin capitò bene e lei fu in buone mani perché a Vicenza gli parlarono di lei il Fogazzaro ${ }^{2}$ e il Senatore Lampertico ${ }^{3}$ e qui all'Antella la sua

(Volti)

Emilia Peruzzi

Aff.ma

Dica al Conte Digerini-Nuti quanto sono grata alla sua amicizia. E lei, caro Fucini, si ricordi di venire qui una domenica dell'Agosto oppure le domeniche del Settembre e Ottobre dal 15 di Settembre in poi.

Tante cose alle Signore

\section{Emilia P.}

Il René Bazin sta a Angers (Maine et Loire) 50 rue des Jardins e mi pare che lei farebbe atto cortese scrivendogli un bigliettino. Egli capisce l'italiano benissimo e gli farà piacere di ricevere una parola da lei.

"Nous nuos promenions (à Vicence), le Senateur Lampertico, le "poête Antonio Fogazzaro et moi. On a proposé la deputation à Mr. "Fogazzaro sans qu'il ait jamais consenti à lasseir voter sur son nom. Mais "le petites charges municipales lui plasent. Il en remplit une demi-“douzaine avec amour et quand je m'étonne, il me cite tranquillement "l'exemple de Renato Fucini, un écrivain de haute valeur comme lui, donc “j'ai lu avec tant de plaisir le poèsies toscanes et les lettres sur Naples, "Fucini qui a commencé par être ingénieur à Florence, et s'est retiré au "fond de sa province natale, du côté d'Empoli, avec un emploi "d'inspecteur primaire. Il y est tres heureux, me dit Fogazzaro - Giosué "Carducci est venu le voir recennement. La campagne, la salut des "concitoyens, la consideration dans tout le municipe et quelquefois la "visite d'un confrêre célèbre d'une autre province italienne: voilà l'idéal "de la vie pour toute une élite, là-bas." ${ }^{4}$

1. René Bazin (Angers 1853 -Parigi 1932). Professore di diritto all'università cattolica della sua città natale, abbandò l'insegnamento per dedicarsi completamente alla letteratura. Tra i suoi romanzi: La famiglia Oberlè (1901), Donatienne (1903), Il grano che cresce (1907). Di grande interesse la biografia Charles de Fuocauld, esploratore del Marocco, eremita del Sahara (1921). L'articolo cui fa riferimento la Peruzzi è Les Italiens d'aujourd'hui. I. Provinces du Nord - La vie provinciale, in «Revue des deux mondes», LXIII, 1 luglio 1893, pp. 47-94.

2. Su Antonio Fogazzaro si veda A. Piromalli, Antonio Fogazzaro, in Letteratura italiana. I minori, cit., vol. IV, pp. 2987-3038; D. Piccioni, L. PICCIOnI, Antonio Fogazzaro, Torino, UTET, 1970.

3. Fedele Lampertico (1833-1906), moralista versatile, soprattutto nello studio della realtà economica in correlazione alla vita fisica ed intellettuale degli aggregati sociali. Seguace del movimento protezionista padovano, fu anche grecista e profondo conoscitore dei poemi omerici. Su di lui si vedano E. Franzina, Il poeta e gli artigiani. Etica del levoro e mutualismo nel Veneto di metà ottocento, Padova, Il Poligrafo, 1988, pp. 14-110; La scienza moderata. Fedele Lampertico e l'Italia liberale, a cura di R. Camurri, Milano, Angeli, 1992.

4. Si tratta di un estratto dell'articolo di Bazin (si veda R. BAzIN, Les Italiens d'aujourd'hui. I. Provinces du Nord-La vie provinciale, cit, pp. 66-67). 


\title{
CLVI
}

\author{
R. Fucini a E. PeruZZi
}

Dianella 29 Agosto 1893

Mia buona Sig. Emilia,

Anche lei deve essere, insieme con molti miei vecchi e cari amici, nella illusione che il Fucini d'oggi sia sempre il Fucini de' bei tempi di Firenze; che gli anni e le traversie della vita abbiano soffiato gelo su tutti meno che su lui. Non è così, Sig. Emilia. Sento che ora non posso assolutamente più scrivere. Feci quel bozzetto per Milano; nel buttarlo giù e nel distrarmi mi pareva che il solito Nume si fosse degnato di scendere a scaldarmi il ferro; ma non era vero.

Leggere il bozzetto fatto e ficcarlo in un fondo di cassetta, fu un atto solo.

Coloro che per espansiva festività dell'animo accennerebbero e forse meriterebbero di non invecchiare mai, invecchiano invece prima e peggio degli altri. Così almeno è accaduto a me.

Al Sig. René Bazin non scriverò. Non per gallofobia, sebbene a questi giorni si potesse, con un certo diritto, tirarne fuori un po' ${ }^{1}$. Non scrivo perché il Sig. Bazin non ha letto né anche una mezza riga delle cose mie. Chi me l'ha detto? Me l'ha detto il mio odorato di vecchio merlo e me l'ha detto una certa conoscenza che ho per pratica del modo col quale i nostri fratelli della Senna scrivono articoli e magari libri sulle cose nostre.

La Sig. ${ }^{a}$ Michelet, la consorte dello storico Michelet ${ }^{2}$, capitata temporibus illis a Firenze scrisse una monografia sui corsi d'acqua della Val di Chiana sopra notizie, illustrazioni e minuziosi schiarimenti che le detti io senza che sapessi un'acca né della Val di Chiana né de'suoi corsi d'acqua. Mi domandò se ne sapevo nulla; per un falso amor proprio e senza immaginarmi le gravi conseguenze della bugia, le dissi di sì, e di quì l'enorme ammasso di bestialità che io le avrò detto e che lei puntualmente scrisse e, quel che è peggio, stampò ${ }^{3}$. Avrei altri esempi, ma s'andrebbe troppo per le lunghe.

CLVI. Dall'aut. In BNCF (CEP, cass. 74, ins. 14, n. 8). Lettera, 4 ff. su 2 cc. Ind.: «Nobil Donna / Emilia Peruzzi / per l'Antella / Firenze». Ann. sulla busta: «Fucini / Perché non / scritto al / Bazin / 30 Agosto 93». Timbro post. di partenza: 30.8.[?]. Timbro post. d'arrivo: 30 AGO 93. Inedita.

1. Fucini allude forse alla "guerra doganale" con la Francia, scatenata nel 1887 da una tariffa doganale apertamente protezionistica approvata in quell'anno dal governo Crispi, e destinata a durare un decennio (cfr. R. Romanelli, L'Italia liberale, in Storia contemporanea, Roma, Donzelli, 1997, p. 183).

2. Athenaïs Mialaret, seconda moglie di Jules Michelet, visse nel Sud della Francia fino ai quattordici anni. Dopo la morte del padre e un grave dissesto finanziario della famiglia, fu costretta a recarsi a Vienna come istitutrice. Fu durante questo soggiorno in Austria che iniziò lo scambio epistolare con lo storico francese, di molto più vecchio della Mialaret, culminato nel matrimonio. Cfr. M. Mùndula, Prefazione a J. Michelet, Lettere ad Atenaide, Roma, Formaggini, 1927, pp. 7-22. Su Jules Michelet (Parigi 1798Hyéres 1874) si vedano G. Monod, Jules Michelet, Paris, Hachette, 1905; ID., La vie et la pensée de Jules Michelet. 1798-1852, Paris, Champion, 1923, voll. 2; D. HalévY, Jules Michelet, Paris, Hachette, 1938; R. BARTHes, Michelet, Napoli, Guida, 1989.

3. L'episodio è ricordato anche in R. FucINI, Acqua passata, cit., pp. 489-499: «Erano ospiti dei signori Sabatier, alla loro villa della Concezione, sui poggi di Fiesole, lo storico Michelet e la sua signora. 
Scrivendogli, non voglio mettere in un impiccio il Sig. Bazin il quale, si sente da lontano un miglio, ha rinvoltato, alla lente per non imbrogliarsi, in tre o quattro luoghi comuni quel bene che dissero di me il Fogazzaro, il Lampertico e la Sig. Emilia, e s'è sentito riavere (mi par di vederlo!) quando s'è accorto d'essere arrivato in fondo.

Queste le mie idee alquanto pessimiste; ma, intendiamoci bene: se la Sig. Emilia desidera questa cosa per averne forse accennato al Sig. René, son capace d'acquistare una risma di carta e di consumarla tutta per una lettera al Sig. Bazin.

Non mi diminuisca la sua benevolenza se le ho scritto una lettera così rabbuffata e tinta di nero.

Suo devot.mo amico

R. Fucini

CLVII

R. Fucini a E. Peruzzi

Dianella 12 Novembre 1893

Mia buona Sig. Emilia,

Appena giuntami la terribile notizia detti sfogo al dolore piangendo l'amico perduto e pensando a Lei. Povero il nostro adorato Genala ${ }^{1}$ ! Che intelligenza ha perduto l'Italia! Che cuore hanno perduto i suoi amici! Io piango a calde lacrime e non so darmi pace.

Serriamo le file e andiamo avanti come si può.

Suo devot.mo

R. Fucini

Capitato lassù per passeggiata da Firenze, fui loro presentato ed ebbi festosa accoglienza. Il Michelet era venuto in Toscana per certi suoi studi; la signora, tanto per impiegar bene il suo tempo, lavorava intorno a una accurata monografia sui corsi d'acqua della Val di Chiana». I Michelet giunsero a Firenze nel settembre del 1870 e vi rimasero fino al giugno del 1871 ; in questo periodo intrecciarono una profonda amicizia con i Sabatier. Durante questo soggiorno in Toscana (i Michelet vissero per alcuni mesi anche a Pisa) Athenaïs Mialaret scrisse una monografia, La Nature, che, tradotta da W. H. Davenport Adams, venne pubblicata in Inghilterra nel 1872 e, successivamente, nel 1893 (London-Edimburgh, Nelson; della prima edizione non si conosce né il luogo di edizione, né l'editore). Non è chiaro se l'opera uscì anche in Francia. Cfr. T. Scharten, Les voyage et séjours de Michelet en Italie, cit., pp. 149-181.

CLVII. Dall'aut. in BNCF (CEP, cass. 74, ins. 14, n. 9). Lettera, 1 f. su 1 c. Ind.: «Nobil Donna / Emilia Peruzzi / per l'Antella / Firenze». Ann. sulla busta: «Fucini / Il Genala / 13 Nov 93». Timbro post. di partenza: 12.[?].[?]. Timbro post. d'arrivo: 13 NOV 93. Inedita.

1. Su Francesco Genala si veda la nota 1 alla lettera CXXvII. 


\title{
CLVIII
}

\author{
E. PeruZZi a R. Fucini
}

Antella 30 Dic. 93

Lei, caro Fucini, ebbe lo slancio di cuore di scrivere a me quando avvenne la disgrazia del caro Amico Genala. Gliene fui grata e non voglio che questo anno finisca senza averglielo detto. Al Manfroni fece maraviglia di non avere una sua parola ${ }^{1}$. Gli dissi della sua lettera a me ma naturalmente non credo che se ne appagasse. Le mando questa Agenda portafoglio e spero che potrà scriverci pensieri sereni. Vi è posto anche per riporre molti fogli denari.

Venga a passare qui una domenica e arrivi avanti le 12 per far colazione con me. Generalmente si parte col tramvaj delle 10,40. Offro a lei e a tutti della cara sua famiglia i miei migliori auguri e sono ora e sempre la sua

Aff.ma

Emilia Peruzzi

\section{CLIX}

R. FuCini a E. PeruZZi

Empoli 11 Gennaio 1894

Mia cara Sig. Emilia,

Anche senza vedere sue lettere e senza che me lo dicesse la Sig. Vittoria, avrei capito che la mia dopo la morte del nostro Genala le ${ }^{1}$ doveva essere stata gradita. Scrissi quella lettera per portare un lieve conforto al suo dolore e per dare sfogo al mio.

Mi sorprende il sentire che i Manfroni non ebbero una mia che impostai insieme con quella per l'Antella dirigendola a Roma, in Via Genova N ${ }^{\circ} 24$. Il servizio della posta, che fra $i$ tanti del Regno sciagurato è uno dei messo peggio, questa volta ha mancato. Mi giustifichi, la prego, con la Sig. a Angiolina e con Mario e ripeta loro quello che io diceva nella lettera: che li invidiavo, in tanto dolore, d'aver avuto la triste soddisfazione di chiudere gli occhi e di deporre un bacio sulla fronte di quel galantuomo senza macchia e senza paura.

CLVIII. Dall'aut. in BRF (FRF, cass. 8, ins. 22). Lettera listata a lutto, 3 ff. su 2 cc. Ind.: «Ill. mo Signore / Renato Fucini». Ann. aut. di Fucini sulla busta: «Emilia Peruzzi». Inedita.

1. Su Manfroni cfr. Introduzione, p. 16, nota 45.

CLIX. Dall'aut. in BNCF (CEP, cass. 74, ins. 14, n. 10). Lettera, 4 ff. su 2 cc. Ind.: «Nobil Donna / Emilia Peruzzi / per l’Antella / Firenze». Ann. sulla busta: «Fucini / L'agenda - la / lettera e il / Genala / 13 del 94». Timbro post. di partenza: 12-1-94. Timbro post. d'arrivo: [?] GEN 94. Inedita.

1. Cassato «sarebbe». 
Peccato che quel cenno biografico non sia continuato fino in fondo! Spero che chi dettò quelle pagine vorrà compiere l'opera ${ }^{2}$. Nel caso, non si scordi lei di me, inviandomene il seguito.

Grazie dell'annuo ricordo. Vorrei poter scrivere ogni giorno del calendario una nota allegra; ma non so se mi sarà possibile come non so se potrò capitar presto all'Antella perché, oltre gli affari, la mia famiglia è in questi giorni un mezzo spedale. Anch'io, che sono il meno peggio, scrivo da una poltrona davanti alla stufa dove, fino da ieri, sono stato traslocato per prevenire, se mi riescirà, questa indecente influenza che da qualche giorno mi minaccia, sibilandomi per il petto peggio d'un serpente a sonagli.

Quanto bene le desidera e le vuole il suo devoto amico

R. Fucini

P.S. Siamo alla guerra. Dio ce la mandi buona! ${ }^{3}$

\section{CLX}

\section{E. Peruzzi a R. Fucini}

Di Villa 17 8bre 94

Caro Fucini, Vi è speranza di vederla domenica prossima e prima che finisca questo tempo bellissimo? Si parte in generale alle 10,40 col tramvaj per arrivare qui a colazione a mezzogiorno. Pare che si pensi sul serio al monumento al Chiarugi ${ }^{1}$. Vi aspira il bravo giovane scultore Vagnetti ${ }^{2}$. La prego di mandarmi un suo biglietto di presentazione per lui dicendomi quali sono le persone alle quali va bene rivolgersi per questa faccenda. Se le fosse impossibile venire domenica prossima, per me va bene anche la domenica 28 .

Scriva le memorie di un Ispettore scolastico; mi ricordi con affetto a sua Moglie e mi abbia sempre per sua

Aff.ma

E. Pzi

2. Non sappiamo di chi Fucini stia parlando.

3. Il postscriptum fa riferimento all'impresa coloniale decisa da Francesco Crispi contro l'Etiopia. È scritto perpendicolarmente sul margine superiore del foglio.

CLX. Dall'aut. in BRF (FRF, cass. 8, ins. 22). Cart. post. Ind.: "All'Ill. mo Signore / Cav. Renato Fucini / Ispettore Scolastico / Empoli per Dianella» (cassato «per Vinci»). Timbro post.: 17 ott. 94. Inedita.

1. Cfr. lettera CLII.

2. Italo Vagnetti, scultore fiorentino. Scolpì il monumento di Ubaldino Peruzzi, scoperto all'Antella l'11 settembre del 1892 (cfr. M. MARCHI, Un centenario da non dimenticare. La morte di Ubaldino Peruzzi all'Antella, in U. Bardi, M. Casprini, M. Marchi, M. Turchi, I Peruzzi all'Antella, cit., p. 11). 


\title{
CLXI
}

\author{
R. Fucini a E. PeruzZi
}

Dianella 23 Ottobre 1894

Mia gentile Sig. Emilia,

Son qui a far dura penitenza dei dolci peccati commessi nell'Agosto. Il mio mese di permesso me lo sono sorseggiato, me lo sono goduto, me lo sono strizzato fino alla buccia come un limone quando abbiamo quello solo e ci sarebbe bisogno d'averne due. Il mediterraneo ${ }^{1}$ non si riconosce più dai solchi che vi ho fatto con l'Iact di Carlo Ginori ${ }^{2}$. Corse a Bastia, cacciate e pescate a Montecristo, gite paradisiache all'Elba, escursioni sulle Alpi Apuane eppoi una punta di tre giorni al Romito ${ }^{3}$ da Sidney Sonnino etc. et. et ${ }^{4} \ldots$ e tutto questo intramezzato da idillici riposi alla incantevole Versiliana di Dreino Digerini ${ }^{5}$. Il canonico Guido Baccelli ${ }^{6}$ mio gran penitenziere, per riparare a tanto male della mia anima non stanca e non sazia, mi ha ordinato undici mesi di espiazione per mezzo di certe prove che, dopo il demonio, non v'è che la burocrazia capace di inventarne delle $\operatorname{cosi}^{7}$ feroci.

E fortuna che non mi lascia vincere dalle lusinghe di Carlo Ginori il quale voleva, in ogni modo, condurmi seco a Costantinopoli dove veramente andò toccando le

CLXI. Dall'aut. in BNCF (CEP, cass. 74, ins. 14, n. 10 bis). Lettera, 4 ff. su 2 cc. Ind.: «Nobil Donna / Emilia Peruzzi / per l'Antella / Firenze». Ann. sulla busta: «Fucini / Verrà nelle / Feste di Natale. / Il monumento al / Chiarugi / 24 8bre 94». Timbro post. di partenza: 23-10-94. Timbro post. di partenza: [?] OTT 94. Inedita.

1. Così nell'originale.

2. Carlo Benedetto Ginori Lisci (Firenze 1851-Monaco di Baviera 1905). Alla morte del padre, ereditò la parte più cospicua delle sue proprietà, compresa la manifattura di ceramiche; spirito irrequieto e bohémien, ne affidò la gestione a Paolo Lorenzini, fratello del celebre Carlo Collodi. Amante dello sport e dell'avventura, nutrì una forte passione per il mare, che lo indusse ad affittare dal Demanio l'isola di Montecristo e farne il punto di ritrovo di nobili e yachtsmen. Intanto la manifattura viveva un periodo di difficoltà; Ginori decise perciò di cederla insieme ad altri immobili. Negli anni successivi avviò altre attività imprenditoriali degne di nota, come una fabbrica di automobili, che però ebbero scarso successo. Proprio la passione per le auto lo indusse a promuovere il Club automobilisti d'Italia (dal 1905 Automobile Club di Firenze).

3. Il Romito è la villa di Sidney Sonnino, situata tra Antignano e Quercianella, in provincia di Livorno.

4. Da "eppoi" si tratta di un'aggiunta inserita da Fucini tra due righe.

5. Sulla villa della Versiliana e Andrea Digerini-Nuti si vedano le note 3 e 4 alla lettera CLIV.

6. Guido Baccelli (Roma 1830- Roma 1916). Intrapresi gli studi di medicina, nel 1848 tentò invano di arruolarsi fra i volontari della prima guerra d'indipendenza (glielo impedì il rettore del collegio presso cui studiava); l'anno successivo, quando la repubblica chiamò alle armi contro i francesi, fu fermato dal padre. Negli anni successivi si dedicò completamente alla medicina, ottenendo nel 1852 e 1853 la laurea in medicina e chirurgia. Fu ministro della Pubblica Istruzione nel governo Cairoli (1881), rimanendo in carica anche durante il governo Depretis; fu poi chiamato allo stesso ministero da Crispi dal 1893 al 1896 e poi da Pelloux nel 1898-1900. Nel 1901 divenne ministro dell'Agricoltura, dell'Industria e del Commercio e si prodigò per la creazione della Galleria d'arte moderna, proponendo di rivalutare l'intero patrimonio artistico, archeologico e naturale della capitale. Sul Baccelli si veda F. LOMBARDI, Guido Baccelli e la sua biblioteca storico-medica, Napoli, Arti grafiche Licenziato, 1964.

7. Così nell'originale. 
principali isole della Grecia, Atene, i porti maggiori dell'Asia minore, e Costantinopoli, e Scutari e...e e che Dio mi il peccato di non essermi lasciato sedurre!

E ora faccio penitenza! E forse me lo merito più per il grosso peccato non commesso che per gli altri piccoli che commisi.

Nei dieci giorni che stetti a Firenze per le conferenze pedagogiche (Dio le perdoni a chi non ha perdonato a me) avevo ideato una corsa all'Antella, che sarebbe stata per me doppiamente piacevole per la simpatica compagnia del Del Lungo ${ }^{8}$; ma ebbi in quei giorni tante seccature, ebbi a correre in tante parti, ebbi a tener dietro a tanti ingrati doveri che quello di una visita a lei dovetti rimandarlo a nuova occasione. Così mi accade spesso anche nella corrispondenza epistolare. Fra un monte di esosissime letteracce, l'ultima alla quale rispondo è quella dell'amico più fido e caro.

Per le feste del Ceppo spero poter venire a Firenze e trattenermi quattro o cinque giorni. E in quei giorni, stia certa, troverò il tempo per salire all'Antella anche se dovessi passare sul cadavere del Ministero dell'Istruzione pubblica, Divisione dell'istruzione primaria e popolare.

Grazie, grazie, grazie.

E il monumento al Chiarugi? Me n'ero dimenticato. L'esecuzione di quest'opera è tanto incerta e tanto lontana che ci sarà tempo, o se ci sarà tempo! di parlarne quando ci vedremo 9 . E quando farò a lei gli auguri per il nuovo anno, dirò: viva, Sig. ${ }^{a}$ Emilia, viva fino a quando sarà innalzata in Empoli una statua al Chiarugi. E sarà questo un augurio di vita così lunga che il povero Noè dovrà ritirarsi nel Limbo fra i marmottini morti nelle fasce. Suo devoto e fedele amico

R. Fucini

8. Isodoro Del Lungo (Montevarchi [Arezzo] 1841-Firenze 1927). Fu legato da una profonda amicizia con Carducci, che esercitò un'influenza decisiva sulla formazione culturale e sugli interessi di Del Lungo. Dal 1863 collaborò a «L'Archivio Storico» di Vieusseux e, in seguito, alla «Nuova Antologia». Per interessamento di Carducci ottenne l'insegnamento delle materie letterarie al liceo di Faenza, passando poi a Casale Monferrato, Siena, Pistoia, Firenze. Dal 1875 abbandonò definitivamente questa occupazione, per dedicarsi del tutto alla ricerca. Fin dalle prime pubblicazioni emerge l'interesse per la letteratura medievale e umanistica, particolarmente per quella toscana. Tra gli studi principali spiccano quelli su Dante (Dell'esilio di Dante [1881]; Dante né tempi di Dante [Bologna. Zanichelli, 1888]...) e su Dino Compagni, di cui curò l'edizione critica della Cronica. Divenne accademico della Crusca (fu compilatore della quinta edizione del Vocabolario e presidente dell'Accademia) e dei Lincei.

9. Il monumento a Vincenzo Chiarugi, che doveva sorgere a Empoli, non venne in realtà mai realizzato. Questa vicenda potrebbe aver ispirato il bozzetto Il monumento contenuto in All'aria aperta. 


\section{CLXII}

\section{R. Fucini a E. PeruZZi}

Dianella 24 Novembre 1894

Mia buona Sig. Emilia,

Vederne cadere due alla volta è troppo. È troppo per il nostro cuore d'amici ed è troppo di fronte alla magra falange di galantuomini intemerati come un Giorgio Pellizzari e un Sansone D'Ancona ${ }^{1}$. Gli avevo visti tutti e due pochi giorni addietro, e tutti e due mi avevano fatto provare una stretta al cuore col loro deperimento spaventevole. Due mesi fa, quando li vidi l'uno a Montepiano e l'altro in Via dell'Oriuolo a Firenze, mi parvero già morti, sebbene la loro anima onesta si affannasse a tenere in piedi quei poveri corpi in sfacelo ${ }^{2}$. Il D'Ancona non l'ho più riveduto dopo quel giorno; al Pellizzari potei, con mia tristissima compiacenza, chiudere gli occhi dopo averlo assistito nei momenti estremi con quel gran bene di figliolo col quale ricambiavo il suo tenero affetto di padre. Ahi, ahi, ahi! Come restiamo pochi a piangere su tanti!

Ma io non voglio rattristarla troppo coll'aggiungere al suo il mio sgomento.

La bontà del suo cuore è tanto sconfinata che non è possibile mai arrivare a conoscerla tutta. In ogni occasione solenne, o lieta o dolorosa che sia, ella non scorda mai questo piccolo, questo modesto, questo affezionatissimo amico suo. Ed io solo lo so se le sono grato. E più invecchio e più sento la dolcezza dell'amicizia, di questa gentile e robusta passione che, sola e quasi ringiovanita da ogni colpo del tempo, sopravvive al naufragio di tutte le altre.

Ma siccome ognuno, per un destino inesorabile, deve esser punito nel suo debole; così io sono condannato a vivere lontano dagli amici miei più fedeli.

Accetterei l'amoroso consiglio che Ella, insieme con altri, mi fa perché scriva e scrivendo mi distragga; ma anche questo il destino non lo vuole. Scrivo anche troppo, ma non quello che vorrei perché quello che vorrei non mi darebbe quello di cui ho bisogno. Messe insieme le piccole rendite patrimoniali con quelle dell'impiego, provvedo comodamente alla mia famiglia. Senza queste ultime dovrei, e non voglio né posso farlo, rinunziare a ceti piccoli agi ai quali ho fatto l'uso e ai quali credo d'aver diritto. E siccome cartelle con premio non ne posseggo, al lotto non gioco, eredità alle viste non ce ne ho punte, così mi son rassegnato e tiro avanti come quel somaro filosofo, il quale, girando il bindolo, trovava una gran consolazione a pensare ai cavalli degli omnibus.

CLXII. Dall'aut. in BNCF (CEP, cass. 74, ins. 14, n. 11). Lettera, 5 ff. su 2 cc. Ind.: «Nobil Donna / Emilia Peruzzi / per l'Antella / Firenze». Annotaz. sulla busta: «Fucini / Il Pellizzari / e il D’Ancona. / Suoi pensieri / 25 Nov. 94». Timbro post. di partenza: 24-11-94. Timbro post. d'arrivo: 25 NOV 94. Inedita.

1. Giorgio Pellizzari fu lettore di anatomia descrittiva, dissertore di anatomia sublime e conservatore del Museo fisiologico del Regio Arcispedale Santa Maria Nuova di Firenze. Su Sansone D’Ancona si veda la nota 1 alla lettera XC.

2. Su Montepiano si veda la nota 1 alla lettera LX. Via dell'Oriuolo è una strada del centro di Firenze, posta vicino a piazza Duomo. 
Ha sentito della povera Calabria ${ }^{3}$ ! Dio ce la mandi buona anche a noi perché c’è da aspettarsene di tutte dopo una siccità della quale né anche il Bodio sa rintracciare l'eguale, col suo tondo occhio di Lince, nei secoli dei secoli che sono passatí.

Se nei nostri aridi colli si va innanzi così un'altra quindicina di giorni, vedo bene che dovrò manomettere un prosciutto per levarmi la sete.

A rivederci a presto. Intanto gradisca i saluti affettuosi della mia Emma e quelli della Maria e della Giorgina Roster le quali sono qui per distrarsi dopo il funesto accaduto.

Nelle feste del Natale verrò, molto probabilmente, per tre o quattro giorni a Firenze, e di lì all'Antella.

Voglia bene al suo fedele amico

R. Fucini

\section{CLXIII}

\section{E. PeruZZi a R. Fucini}

Antella 31 Dic. 94

Neppure i giorni delle vacanze mi hanno procurato il piacere di rivederla. Vittoria ${ }^{1}$ mi scrisse di una sua indisposizione ed io spero che ora ella sarà perfettamente ristabilito salutando l'anno nuovo come si saluta una conoscenza nuova e che in se racchiude molte cose ignote. Le mando l'agenda alla quale ella confiderà i suoi pensieri ricordando la sua aff. ma Emilia che manda affettuosi auguri a lei, a sua moglie e a tutti i suoi.

3. Fucini allude forse al terremoto che squassò la Calabria e la Sicilia il 17 novembre 1894. Cfr. «La Nazione» del 18 novembre di quell'anno.

4. Sulla siccità che colpì l'Italia si veda "La Nazione» del 16 novembre 1894. Luigi Bodio (Milano 1840-Roma 1920). Laureatosi in legge a Pisa, nel 1862 soggiornò a Parigi, dove incontrò insigni statisti ed economisti. Ottenuta l'abilitazione alla professione forense, insegnò materie economiche e statistiche; fu designato a presiedere alla direzione dei servizi statistici del Regno, lavorando anche al censimento demografico del Regno d'Italia del 1871 predisponendo le istruzioni preliminari ed elaborando le relazioni finali. Fu tra i fondatori dell'Istituto internazionale di statistica. Essendosi a lungo interessato dei fenomeni migratori, nel 1901 venne preposto all'organizzazione del Commissariato per l'emigrazione, di cui diresse l'attività fino al 1904.

5. Corregge «marimettere».

CLXIII. Dalla riproduz. fot. in G. RossI, Salotti letterari in Toscana, cit., fig. 81, p. 175. Bigl. vis. listato a lutto.

1. Su Vittoria Altoviti Avila si veda la nota 4 alla lettera LXXIV. 


\title{
CLXIV
}

\author{
R. FuCini a E. PERuZzi
}

Dianella 6 Gennaio 1895

Mia gentile Sig. Emilia,

Ed ecco come vanno le cose di questo mondo. Il 23 del mese scorso m'ero preparato per venire a Firenze a festeggiare l'onomastico della Sig. ${ }^{a}$ Vittoria; ma mi saltò addosso una bella febbre e dovetti rinunziare a quel piacere e anche a quell'altro di una gita all'Antella, che avevo messo nel mio bilancio preventivo.

Stamani dovevo venire con l'Emma a fare la Befana dai Roster; ed ecco che la bianca fata del nord ci ha sequestrati a Dianella. Meno male che oggi ho da consolarmi con questo spettacolo, per me sempre stupendo, che mi rallegra malinconicamente e che, anche ora da vecchio, mi fa sognare come da fanciullo.

Grazie dell'agenda. Se la Provvidenza m'assiste, voglio empirla di ricordi piacevoli. Mi riescirà? La regola del tre nella quale, col passare degli anni, sempre più mi approfondisco, mi dice di dubitarne.

Che le è parso di quei martelliani calabresi? Al teatro d'Empoli, recitati discretamente da un dilettante truccato da cittadino della Sila, fecero grande effetto e ottennero largo premio di commozione, di applausi e, quel che più conta, di offerte per i poveri danneggiati dal terremoto ${ }^{1}$. Io non ci ero: me l'hanno raccontato.

Mi scusi se scrivo poco e in fretta. Approfittando della dolce prigionia alla quale mi condanna la neve, dò mano a purgare il mio tavolino da una montagna di orrende statistiche per empirne le insaziabili canne del Bodio e i patriottici banchi dei salumai.

Da tutti e da me saluti e augurj affettuosissimi.

Suo devoto amico

R. Fucini

CLXIV. Dall'aut. in BNCF (CEP, cass. 74, ins. 14, n. 12). Lettera, 4 ff. su 2 cc. Ind.: «Nobil Donna / Emilia Peruzzi / per l'Antella / Firenze». Ann. sulla busta: «Fucini / Perché non venne / all'Antella - / I versi del terremotato - / Ringrazia / 8 del 95». Timbro post. di partenza: 7-1-95. Timbro post. d'arrivo: 8 GEN 95. Inedita.

1. Si tratta di una poesia in versi martelliani, Il superstite calabrese, scritta da Fucini e recitata come prologo nel Regio Teatro di Empoli la sera del 30 dicembre 1894 in occasione di uno spettacolo di beneficenza a favore delle vittime del terremoto in Calabria (vedi E: Caramelli, Figure di altri tempi, cit., p. 37). 


\title{
CLXV
}

\author{
R. Fucini a E. PeruZZi
}

Dianella 2 Gennaio 1896

Gentilissima Sig. Emilia,

Ella mi ha prevenuto col suo dono cortese ${ }^{1}$. Mi ha prevenuto di poche ore e per una combinazione. L'ultimo dell'anno avevo sul tavolino una lista di nomi per altrettante lettere, ma invece consumai quella giornata (non rida!) a scrivere una scena comica per i burattini che saranno fatti nel carnevale al Circolo degli Artisti di Firenze ${ }^{2}$. Preso per i capelli dalla ispirazione, m’arrabattai tutto quel giorno per concludere da ultimo poco o nulla. E per venire subito a cose più solide, sappia che fino dal 15 del mese passato ho riportato la mia residenza a Firenze e ho preso casa in Via Pinti $\mathrm{N}^{\circ}$ 64. Ora mi trovo qui a passare le feste in famiglia. Domenica sarò di nuovo in Firenze. Come mai questa mia risoluzione? Mille cause e tutte dolorose mi hanno sbalestrato di nuovo nella gran città. Piglio il mondo come viene e mi conforto pensando che forse non sarà per il mio male uscire, prima d'esser vecchio davvero, dalla eccessiva solitudine della campagna o dal disumano consorzio di un borgaccio di bottegai dei quali l'ideale più nobile è quello di far quattrini.

Il giorno stesso che arrivai a Firenze, incontrato il Linaker, lo abbordai per invitarlo a far subito una passeggiata all'Antella ${ }^{3}$; e da lui fui raffreddato con la notizia che ella era a Viareggio ${ }^{4}$. Avevo saputo dalla Cont. Alessandri del suo soggiorno costà, ma credevo che si trattasse di un semplice caso di bagnature e basta ${ }^{5}$. Invece sento che ella passerà costà tutto l'inverno. È vero?

CLXV. Dall'aut. in BNCF (CEP, cass. 74, ins. 14, n. 12 bis). Lettera, 4 ff. su 2 cc. Ind.: «Nobil Donna / Sig Emilia Peruzzi / Piazza d'Azeglio No 18 / (Lucca) Viareggio». Ann. sulla busta: «Fucini / Ringrazia. E / venuto a stare / a Firenze. Vittoria / 3 del 96». Timbro post. di partenza: 2-1-96. Timbro post. d'arrivo: 3-1-96. Inedita.

1.La Peruzzi inviava ogni anno, per Natale, un dono a Fucini, di solito un'agenda. Si veda, la lettera precedente.

2. Si tratta forse di Si salvi chi può, che, secondo la Caramelli, venne rappresentata il 21 marzo 1896 al Circolo degli Artisti (vedi, E. Caramelli, Figure di altri tempi, cit., p. 37). Il Circolo degli Artisti venne fondato nel 1878. Ebbe sede in Borgo degli Albizi (palazzo Matteucci-Monsalvo), almeno fino al 1881, quando si trasferì in via de'Servi (palazzo Tedaldi-Naldini). Dal 1887 al 1898 i soci si riunirono in palazzo Pucci, dove ebbero luogo esposizioni artistiche e conferenze. Cfr. V. VANnUCCI, Istituzioni fiorentine, cit. , pp. 305-317.

3. Su Arturo Linaker si veda la nota 5 alla lettera CVI.

4. La signora Emilia, molto malata e cieca, trascorreva lunghi periodi a Viareggio. Cfr. M. P. CucColi, Emilia Toscanelli Peruzzi, cit., p. 209. Steinmann ci informa che la Peruzzi soggiornò circa un anno e mezzo a Viareggio, dove aveva preso in affitto, in piazza D'Azeglio, una casa provvista di alcune camere per ospitare gli amici (si veda E. Steinmann, Emilia Peruzzi, cit., p. 220).

5. La famiglia Alessandri affonda le proprie origini nel Medioevo, quando Alessandro e Bartolomeo, figli di Niccolò Degli Albizi, ottennero dalla Signoria il permesso di mutare il cognome (1372). Nel corso del ' 400 vennero stretti legami di parentela con i Medici, cosa che comportò l'assunzione di cariche pubbliche. Nei secoli successivi, tuttavia, gli Alessandri persero molto del potere acquistato, pur rimanendo 
Quanto cambiata la nostra Firenze! Quanti amici morti, quanti lontani, quanti invecchiati da destare pietà! E la Sig. ${ }^{a}$ Vittoria ${ }^{6}$ ? Quanta pena per quella infelicissima donna! Che sarà quel suo male? ? Il Bargioni e il Roster si stringono nelle spalle; altri

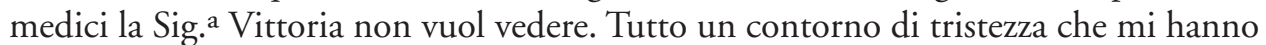
fatto malino<ni $>$ ci questi primi giorni della terza maniera di Firenze per me un tempo allegra e fiorita di speranze e di gioconde illusioni ${ }^{8}$.

Quanto mi rincresce di non poterla presto rivedere. Prima la Sig. ${ }^{a}$ Vittoria era il nostro anello di congiunzione per notizie e saluti frequenti. Ora quell'anello è interrotto. Speriamo che lo sia per poco.

Grazie, mia buona Sig. ${ }^{a}$ Emilia, grazie del regalo e della memoria. Io Le ricambio tutto coi miei più lieti e fervidi augurj per il nuovo anno e per sempre.

Suo devoto amico

R. Fucini

\section{CLXVI}

E. Peruzzi a R. Fucini

Viareggio 14 agosto 1896

18 Piazza d'Azeglio

\section{Caro Fucini}

Il pensiero della nostra Vittoria trattenne me dallo scriverle, ma dire il vero avvenuta la disgrazia, aspettavo una sua parola che facesse eco a quelle che prorompevano dall'anima mia. Fu per me una tenera sorella che io rimpiangerò finché avrò vita. E ora, caro Fucini, mi dirigo a Neri Tanfucio chiedendo una poesia da mettersi nel numero unico che sarà stampato qui per il 24 Agosto a benefizio dello Spedale in parte costruito ${ }^{1}$. Qualunque cosa sia sarà una perla forse pure in versi o in prosa. Il mare, i bagni, le troppe baracche che troppo nascondono la vista delle onde, i suoni degli organini e

una delle casate fiorentine più importanti e antiche. Si veda D. TiribILLI-GIULIANI, Sommario storico delle famiglie celebri toscane compilato da Demostene Tiribilli-Giuliani di Pisa riveduto dal Cav. Luigi Passerini, Firenze, Diligenti, 1862, vol. I, pp. 30-31; L. GINORI-LisCI, I palazzi di Firenze nella storia e nell'arte, cit., vol. II, pp. 551-558.

6. Su Vittoria Altoviti Avila si veda la nota 4 alla lettera LXXIV.

7. Su Giorgio Roster si veda la nota 2 alla lettera XIV; sul dottor Bargioni cfr. invece la nota 1 alla lettera CXI.

8. Fucini si riferisce ai suoi due precedenti soggiorni a Firenze, il primo iniziato durante il periodo della capitale e conclusosi nel 1878 (cfr. lettera XCIV), il secondo dal 1886 al 1889 (si vedano le lettere CXXIV e CXXXVI).

CLXVI. Dall'aut. in BRF (FRF, cass. 8, ins. 22). Lettera listata a lutto, 3 ff. su 2 cc. Inedita.

1. Non siamo purtroppo riusciti a identificare il numero unico cui allude donna Emilia. Che la nobildonna si fosse, tuttavia, impegnata in questa iniziativa benefica è attestato da M. M. Manfroni, Prologo a E. Homberger, M.M. Manfroni, Il Bamboccio, cit., p. 121. 
quelli degli organi delle giostre che suonano tutti a un tempo, assordano e par di essere la sera nella torre di Babele. Dunque ella canti in versi o in prosa e quanto alla prosa, sappiamo che presto verrà in luce un volume preziosissimo ${ }^{2}$.

Mille saluti alla gentile sua Moglie e risposta presto e bene alla voce della carità che si rivolge a lei per mezzo della sua

Aff.ma

Emilia Peruzzi

\section{CLXVII}

E. Peruzzi a R. Fucini

Antella 10 Dic. 96

Un caro libro con parole dolci, affettuose e ricche di ricordanze ${ }^{1}$ : Quando potremo rivederci? Le domeniche è libero la mattina o la sera? Potrebbe venire domenica prossima o l'altra che è il 20? E la mattina fra le 12 e il tocco per la colazione, o nelle ore pomeridiane per desinare con noi?

Tante cose e grazie di cuore per questo dono graditissimo dalla sua

Aff.ma

Emilia Peruzzi

\section{CLXVIII}

\section{E. Fucini a E. PeruZzi}

\section{Gentilissima e cara Sig. ${ }^{\text {a }}$ Emilia}

Renato non è in Firenze e tornerà Lunedì o Martedì. Colgo quest'occasione e rispondo io alla sua gentilissima letterina che ho aperto, come apro tutte le lettere di

2. Il volume in questione è All'aria aperta, edito da Bemporad nel 1897. Si veda la lettera successiva.

CLXVII. Dall'aut. in BRF (FRF, cass. 8, ins. 22). Lettera listata a lutto, 2 ff. su 2 cc. Inedita.

1.Si tratta probabilmente di una prima copia di R. Fucini, All'aria aperta. Scene e macchiette della campagna toscana, Firenze, Bemporad, 1897. Si veda un biglietto di Carducci a Fucini in cui il poeta maremmano lo ringrazia per il libro inviatogli: «Roma 18 dicembre 1896. Caro Fucini, ho tardato il ringraziamento per farlo più di cuore e d'informata coscienza. E perciò ho letto e a punti riletto la tua vivacissima prosa, da cui c'è sempre da imparare anche per l'elocuzione. Tuo Giosué Carducci» (cfr. L. G. SBrocchi, Renato Fucini. L'uomo e l'opera, cit., p. 56).

CLXVIII. Dall'aut. in BNCF (CEP, cass. 74, ins. 14, n. 14). Bigl. vis. intestato "Emma Fucini". Ind.: «Alla Nobil Donna / Sig. ra Emilia Peruzzi / Borgo dei Greci / No 10 per l'Antella». Ann. sulla busta: «Sig.ra Fucini / Suo marito è assente / Il libro "all'aria aperta" / La perdita della cara / Vittoria. / notizie / 13 Dic. 96». Inedito. 
mio marito quando egli è assente, per ordine suo. Speriamo che il libro (All'aria aperta) incontri <successo> e che Renato calmi la sua agitazione per l'esito che egli teme sempre incerto, come ha temuto per tutti i suoi scritti! Cara Signora Emilia, la ringrazio del bene che lei ha sempre voluto a Renato e l'assicuro che ne è contraccambiata. Appena egli avrà qualche ora disponibile, verrà molto volentieri a farle una visita $\mathrm{e}$ io mi farò un dovere di rammentarglielo. Credo che ai poeti sia lecito avere un rammentatore!

La perdita della nostra Vittoria mi ha tolto anche l'occasione di parlare di lei, come si faceva spesso, ed è troppo doloroso vedersi portar via così le amiche care come lo era lei! Giorgio stà abbastanza bene e trova compenso nell'affetto della mamma e della sua figliolina ${ }^{1}$. Le mie figliole stanno bene e mi hanno fatto nonna di cinque cari nipotini che sono la mia delizia. La saluto caramente e la prego di volere un po' di bene alla sua aff. amica

Emma Fucini.

Firenze 12.12.96

\section{CLXIX}

R. Fucini a E. Peruzzi

Firenze 18 Decembre 1896

Gentilissima Sig. ${ }^{a}$ Emilia,

Grazie del Suo cortese invito e della buona amicizia che mi conserva, sempre viva e fresca al pari della mia. Sarei già da qualche tempo venuto a salutarla se, appunto, non avessi saputo che i suoi medici le avevano accennato di non ricevere troppa gente e, in specie, persone che avrebbero potuto destarle troppo viva impressione. Peccando forse di orgoglio, mi ero creduto nel numero di queste e guardavo l'Antella con desiderio, ma da lontano.

Più presto che mi sarà possibile verrò a salutarla e, se ci troviamo d'accordo, verrò col Linaker ${ }^{1}$. Ho detto - più presto che mi sarà possibile - poiché in questi giorni ho dinanzi a me un tal cumulo di seccature da non saper come principiare a smonticarlo. Le feste natalizie col loro corteggio di rompicapi, le feste della esposizione nel vortice delle quali mi trovo involontariamente trascinato, eppoi l'Uffizio, eppoi le gite per il Circondario, eppoi...eppoi... Le basti che sul mio taccuino, dopo aver scassato il suo nome per questa lettera da scriverle, trovo segnate, fra grosse e piccine, quarantadue cose da levar di mezzo prima che finisca la nuova settimana.

1. Si tratta di Giorgio Roster, fratello di Emma Fucini (su di lui cfr. la nota 2 alla lettera XIV).

CLXIX. Dall'aut. in BNCF (CEP, cass. 74, ins. 14, n. 15). Lettera, 4 ff. su 2 cc. Ind.: «Nobil Donna / Emilia Peruzzi / Antelld». Ann. sulla busta: «Fucini / Perché non venne / Le sue occupazioni / 19 Dic. 96». Inedita.

1. Su Arturo Linaker cfr. lettera CVI, nota 5. 
Si vive una vita da cani. E questo perpetuo logorio è per me addirittura insopportabile. Se non vado a domiciliarmi in un'isola deserta dell'Oceania, lo scriva come un miracolo. Che del resto sto molto bene di membra e di spirito e tollero con una rassegnazione che par contentezza tutto quello che Dio mi manda.

La mia Emma la saluta e la ringrazia del biglietto. A rivederci presto e creda sempre alla devota e sincera amicizia del Suo

R. Fucini

\section{CLXX}

\section{E. PeruZZi a R. FuCINI}

Antella 30 Dic. 96

Scriva i suoi bei pensieri in questo libretto che le sarà, spero, fido compagno. Tanti auguri a sua Moglie ed a lei dalla vostra

Aff.ma

Emilia Pzi

CLXXI

R. Fucini a E. PeruZZi

Firenze 1 Gennaio 1897

In ogni pagina di questo taccuino segnerò un pensiero che suoni lieti augurj per Lei. Me ne mandi di questi taccuini, me ne mandi per molti anni ancora.

Grazie, Signora Emilia, grazie di cuore.

Suo devot.mo

R. Fucini

CLXX. Dall'aut. in BRF (FRF, cass. 8, ins. 22). Bigl. vis. listato a lutto e intestato "Emilia Peruzzi". Ann. aut. e a lapis di Fucini: «In ogni pagina di questo taccuino segnerò un pensiero che suoni belle cose per lei» (cfr. lettera CLXX).

CLXXI. Dall'aut. in BNCF (CEP, cass. 74, ins. 14, n. 16). Bigl. vis. Ind.: «Nobil Donna / Emilia Peruzzi / Antella». Ann. sulla busta: «Fucini / Ringrazia / 1 del 97». Inedito. 


\title{
CLXXII
}

\author{
E. Peruzzi a R. Fucini
}

Firenze, Villa dell'Antella

28 Dic. 97

EMILIA PERUZZI sempre molto sofferente, manda a lei e a tutta la sua famiglia i suoi memori e AMICHEVOLI AUGURI.

\section{CLXXIII}

\section{R. Fucini a E. PeruZZi}

Gentil.ma Sig. Emilia,

Non è tanto gradito il consueto ricordo quanto è gradito per me l'attestato che esso mi porta delle sue migliorate condizioni di salute. Dio voglia che l'Antella possa un giorno ritornare il geniale ritrovo dell'amicizia e della cordialità! Così potesse anche tornarvi l'antica allegria; ma quella ormai non più, perché troppe e troppo a noi care le ombre degli amici caduti che vi aleggiano intorno.

Non sono più allegro nemmeno io che lo ero tanto. Se ne ricorda, Signora Emilia? Anche per me il buono è passato e aspetto con la coda fra le gambe quello che verrà.

Avevo trovato distrazione opportuna nella vita di Firenze, il Codronchi vi ha messo riparo rimandando tutti gli ispettori al Circondario ${ }^{1}$. Ed io ho dovuto, fino dal Novembre, abbandonare al buio e alla polvere la mia bella casina di Via Pinti e rintanarmi a Dianella dove la solitudine fa delle brecce desastrose nella serenità del

CLXXII. Dall'aut. in BRF (FRF, cass. 8, ins. 22). Bigl. vis. listato a lutto e intestato "Emilia Peruzzi". L'indirizzo e gli auguri sono prestampati. Inedito.

CLXXIII. Dall'aut. in BNCF (CEP, cass. 74, ins. 14, n. 17). Lettera, 3 ff. su 1 c. Ind.: «Nobil Donna / Emilia Peruzzi / per l'Antella / Firenze». Ann. sulla busta: «Fucini / Ringrazia. Non è / più allegro. Il Co / dronchi lo riman / dò a Empoli / 16 Gennaio 98». Timbro post. di partenza: 15-1-98. Timbro post. d'arrivo: 1698 . Inedita.

1. Fucini si era trasferito a Firenze da Empoli tra il dicembre 1895 e il gennaio 1896. Si veda la lettera CLXV. Giovanni Codronchi Argeli (Imola [Bologna] 1841-Roma 1907). Studiò alla facoltà legale presso l'Università di Bologna, laureandosi nel 1862. Iniziò a occuparsi attivamente di politica, intraprendendo una carriera di spicco nelle amministrazioni locali. Dopo l'avvento della Sinistra, sostenne la necessità di larghe intese fra i due schieramenti di fronte ai pericoli più gravi, e in più occasioni difese il "trasformismo" con la necessità di superare rancori dannosi per poter contrastare l'estrema Sinistra e per migliorare l'indirizzo della politica estera nazionale. Nel 1889 venne nominato da Crispi prefetto di Napoli e, poco dopo, senatore. L’anno successivo, alla vigilia delle elezioni, venne trasferito alla prefettura di Milano. Nel 1897 divenne ministro della Pubblica Istruzione. Rifiutò il posto di ambasciatore e il governatorato dell'Eritrea, e da allora si dedicò esclusivamente all'attività di senatore. 
mio spirito. Spero di tornare presto in città, ma quando e per quanto tempo non lo so. Se a Firenze saprò che i medici le hanno permesso di riaprire la sua porta agli amici, verrò con gioia a salutarla.

Le invio frattanto i miei ringraziamenti e i miei augurj più cordiali.

Suo devot.mo amico

R. Fucini

\section{CLXXIV}

R. Fucini a E. PeruZzi

Firenze 27 Aprile 1898

Pieno di care e ormai lontane memorie, oggi, mentre Firenze innalza una statua all'uomo di cui Ella fu degna consorte e ispiratrice ${ }^{1}$, il mio pensiero viene da Lei, buona

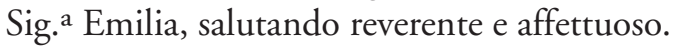

Il suo amico

R. Fucini

\section{CLXXV}

E. Peruzzi a R. Fucini

Firenze, Villa dell'Antella $30 / 4 / 98$

\section{EMILIA PERUZZI}

Profondamente commossa ringrazia sentitamente tornando col pensiero a un passato purtroppo per sempre perduto.

CLXXIV. Dall'aut. in BNCF (CEP, cass. 74, ins. 14, n. 18). Bigl. Ind.: «Nobil Donna / Emilia Peruzzi / Antella». Ann. sulla busta: «Fucini / Per il 27 / 27-4-98». Inedito.

1. Il 27 aprile 1898, in occasione dell'anniversario della cacciata del granduca Leopoldo II (1859), alla presenza del Re furono scoperti, in Piazza dell'Indipendenza a Firenze, i monumenti a Bettino Ricasoli e Ubaldino Peruzzi (cfr. G. Spadolini, Firenze fra '800 e '900, cit., p. 135).

CLXXV. Dall'aut. in BRF (FRF, cass. 8, ins. 22). Bigl. vis. listato a lutto e intestato "Emilia Peruzzi”. Ind. stampato. Inedito. 


\section{CLXXVI}

R. Fucini a E. PeruZZi

Gentil.ma Sig. Emilia,

Firenze 1 Gennaio 1899

Col passare degli anni molti ricordi si annebbiano nella mia memoria; ma non si annebbiano quelli dell'Antella i quali, sorridendomi da lontano, si stringono e si confondono con le più dolci rimembranze della mia lieta giovinezza.

Quanti debiti con lei! Sento che la mia gratitudine, per quanto sconfinata, non basterà mai a saldarli.

Questi sentimenti vorrei poterle esprimere con la voce, e aspetto con vivo desiderio un cenno che mi inviti a farlo. Sarà vicino questo momento? Lo spero.

Le mando dal cuore augurj fervidissimi.

Suo devoto amico

R. Fucini

CLXXVI. Dall'aut. in BNCF (CEP, cass. 74, ins. 14, n. 19). Lettera, 2 ff. su 2 cc. Ind.: «Nobil Donna / Emilia Peruzzi / per / L’Antella». Ann. sulla busta: «Fucini / Auguri / 2 del 99». Inedita. 


\section{APPENDICE}

\section{CLXXVII}

\section{R. Fucini a E. PeruZZI}

Verrò, verrò, verrò.

E a che servono le dighe, gli argini, le sassaie, i puntoni? Ella è un fiume un torrente, un uragano, un tifone che tutto abbatte, sradica e porta con se.

Verrò, verrò, verrò.

Ma la responsabilità dell'atto che sto per commettere verso questi altri amici tutta ricada sopra di Lei. Ah! Genala, Genala!... ${ }^{1}$

\section{CLXXVIII}

Accetto con piacere l'invito per domani domenica e la ringrazio.

\section{CLXXIX}

\section{R. Fucini a E. PeruZZI}

Ed io ringrazio lei e il Sig. Ubaldino dei loro grazie i quali sarebbero stati, per parte mia, più meritati se un certo timor panico non mi avesse impedito di colorire quelle

CLXXVII. Dall'aut. in BNCF (CEP, cass. 74, ins. 8, n. 1). Bigl. vis. intestato "Ing. Renato FuCINI". S. d. Inedito.

1. Su Francesco Genala cfr. la nota 1 alla lettera CXXVII.

CLXXVIII. Dall'aut. in BNCF (CEP, cass. 74, ins. 8, n. 2). Bigl. vis. adespota intestato "ING. RenATO FuCINI”. Data (errata; il 25 gennaio 1872 era mercoledi), non aut. e a lapis: 25.1.72?. Inedito.

CLXXIX. Dall'aut. in BNCF (CEP, cass. 74, ins. 8, n. 3). Bigl. vis. intestato "Ing. Renato FuCinı". S. d. Inedito. 
piccole cose un po' meglio. Le Domeniche le ho tutte impegnate con la mia famiglia e Giovedì pure dovrò rinunziare all'Antella per Empoli dove sono atteso dagli amici e parenti miei alla fiera ${ }^{1}$.

Un saluto di cuore da suo devot.mo amico

R. Fucini

\section{CLXXX}

\section{R. Fucini a E. PeruZZi}

Ieri sono stato alla Cava dove mi sono immensamente divertito, ma con mio dispiacere non potei condurre la mia Emma perché un poco incomodata ${ }^{1}$. Oggi non si sente punto meglio di ieri e io non vorrei lasciarla anche oggi: dico lasciarla perché non ci vediamo altro che all'ora di desinare, perciò mi perdonerà, la Sig. ${ }^{a}$ Emilia se questa volta non accetto il gentile invito.

La ringrazio e la saluto distintamente

Lunedì

\section{CLXXXI}

\section{R. Fucini a E. PeruzZi}

Non potendo in persona prendo le mie rassegne con questo biglietto. Stasera non ci vedremo perché vado ad accompagnare la mia famiglia in campagna. Lunedì o Mercoledì alla più lunga farò bella mostra della mia rispettabile individualità nel suo salone.

1. La fiera di Empoli si tiene ogni anno a fine settembre, fin dal 1548. In origine si trattava di una fiera per la compravendita del bestiame. Cfr. Guida turistica della città di Empoli, a cura di A. Morelli, Bologna, S.T.E.B., 1959, pp. 107-108.

CLXXX. Dall'aut. in BNCF (CEP, cass. 74, ins. 8, n. 4). Bigl. vis. intestato "InG. Renato Fucinı". S. d. Inedito.

1. Si tratta della villa della Cava, vicino Pontedera, di proprietà della famiglia Toscanelli.

CLXXXI. Dall'aut. in BNCF (CEP, cass. 74, ins. 8, n. 5). Bigl. vis. intestato "Ing. RenATo FuCini". S. d. Inedito. 


\title{
CLXXXII
}

R. FuCini a E. PERUZZI

Sta bene per domenica prossima all'Antella. La saluto rispettosamente.

\section{CLXXXIII}

\author{
R. Fucini a E. PeruZZi
}

Gent.li.ma Sig. Emilia,

Debbo rinunziare a quanto ella mi offre per domani perché ho dei parenti a desinare da me.

Mi dispiace, ma come si fa? Mi scusi ed accetti i buoni augurj del suo devot.mo R. Fucini

\section{CLXXXIV}

Gentil.ma Sig. Emilia,

Senza che io le accenni le cagioni ella mi perdonerà il non essermi fatto vivo dopo il suo gentile invito per la passeggiata all'Incontro. Deploriamo insieme la enorme sventura toccata al povero Roster ed all'intera sua famiglia della quale partecipo alle gioje ed alle lacrime più che come amico come fratello ${ }^{1}$. La saluto affettuosamente

R. Fucini

CLXXXII. Dall'aut. in BNCF (CEP, cass. 74, ins. 8, n. 6). Bigl. vis. intestato "ING. Renato FuCINI". S. d. Inedito.

CLXXXIII. Dall'aut. in BNCF (CEP, cass. 74, ins. 8, n. 7). Bigl. vis. S. d. Inedito.

CLXXXIV. Dall'aut. in BNCF (CEP, cass. 74, ins. 8, n. 8). Bigl. vis. intestato "RenATo FuCinI". S. d. Inedito.

1. Su Giorgio Roster si veda la nota 2 alla lettera XIV. 


\title{
CLXXXV
}

\author{
R. Fucini a E. Peruzzi
}

Mille complimenti alla Sig. ${ }^{a}$ Emilia con preghiera di farne parte al Sig. Ubaldino.

\section{CLXXXVI}

\section{R. Fucini a E. PeruZZi}

Può figurarsi se ne sono dispiacentissimo! Ma è impossibile che stasera io venga al Niccolini.

La ringrazio di tutto cuore e sono mortificato d'averle dato il disturbo di scrivermi inutilmente, sapendo quanto ella sia occupata.

Martedì

\section{CLXXXVII}

\section{R. Fucini a E. PeruZZi}

È impossibile! Passando da Empoli con la mia famiglia per andarmene a Firenze, trovo il suo biglietto dove mi prega di impostare una risposta sonettata per oggi stesso...

O come devo fare? Qui ci vorrebbe davvero un improvvisatore ma di quelli che sapessero isolarsi anche in tempo che si fa un viaggio con moglie, figlioli, cani, bauli ed altre gioje de'padri di famiglia che stendono o dopo la villeggiatura o dopo la bagnatura. Me ne dispiace tanto tanto; ma è cosa da rimettersi a quest'altro onomastico ${ }^{1}$. Mi piace annunziarle che il mio scherzo comico, se non sono stato ingannato dai referti, ha avuto un esito felice alle Mozzete $^{2}$.

CLXXXV. Dall'aut. in BNCF (CEP, cass. 74, ins. 8, n. 9). Bigl. vis. intestato "Ing. Renato FuCinI". S. d. Inedito.

CLXXXVI. Dall'aut. in BNCF (CEP, cass. 74, ins. 9, n. 4). Bigl. vis. adespota intestato "ING. ReNATO FuCINI". Data (errata; il 19 aprile 1872 era venerdi), non aut. e a lapis: 19.4.72. Inedito.

CLXXXVII. Dall'aut. in BNCF (CEP, cass. 74, ins. 9, n. 14). Bigl. vis. intestato "RENATO FUCINI". S. d. Inedito.

1. Emilia Peruzzi aveva evidentemente chiesto a Fucini di comporre dei versi in occasione di S. Carlo (cfr. nota 6, lettera LXXIII).

2. Si tratta di villa Corsini delle Mozzete, nei pressi di Firenze. Sullo «scherzo comico» cfr. P. CARLuCCI, Lettere di Sidney sonnino ad Emilia Peruzzi, cit., p. 97: «Ho sentito gram lodi di una scena comica del Fucini, rappresentata alle Mozzete alla Villa del Marchese di Laiatico [...]». 
Che l'ha ricevuta una mia lettera nella quale ringraziavo Lei e il Sig. Ubaldino per l'invito alla Vendemmia ${ }^{3}$ ? (la risposta a voce a Firenze)

Complimenti e saluti tutti codesti signori e specialmente poi da parte di mia moglie, la Sig. ${ }^{a}$ Vittorina e voglia sempre credere alla perfettissima stima e simpatia del suo devoto servo

R. Fucini

\section{CLXXXVIII}

\section{E. PeruZZi a R. Fucini}

Venerdì

Ho fatto gran festa al suo librino e me lo son portato in carrozza come una cara compagnia ${ }^{1}$. Il Bonghi ne ha letto con me divertendosene molto ${ }^{2}$. E del poeta inaspettato che ne dice?

Sua

Emilia

\section{CLXXXIX}

\section{E. Peruzzi a R. Fucini}

\section{Giovedì sera}

Domani sera Venerdì l'aspettiamo. Se vuol dirlo a Giorgio Roster ci pensi Lei che lo ha vicino. ${ }^{1}$. Venga all'ora che le piace ma leggeremo alle 9 perché il Giarrè non può prima ${ }^{2}$.

Mille cose e mille grazie.

E.

3. Si veda la nota 1 alla lettera XL.

CLXXXVIII. Dall'aut. in BRF (FRF, cass. 8, ins. 19). Lettera intestata "EP", 1 f. su 2 cc. Ann. aut. e a lapis di Fucini: «Peruzzi». Anno, non aut.: 1877?. Ind.: «Ill. mo Sig. re Fucini / Ing.re /Al Municipio». Inedita.

1. Si tratterà probabilmente dei Cento sonetti in vernacolo pisano, editi a Firenze da Pellas nel 1872. L'anno indicato sulla lettera sarebbe dunque errato (ci induce a pensarlo il fatto che la lettera sia stata indirizzata al Municipio, dove Fucini lavorava come ingegnere). Difficile pensare, invece, che si tratti dei Cento sonetti in vernacolo pisano. Nuove poesie, editi da Prospero Barbèra nel 1876 dato che, proprio in quell'anno, a causa della caduta della Destra, i rapporti tra Bonghi e i Peruzzi si erano interrotti.

2. Su Bonghi si veda la nota 4 alla lettera XXXV.

CLXXXIX. Dall'aut. in BRF (FRF, cass. 8, ins. 19). Lettera intestata "EP", 2 ff. su 2 cc. Ann. aut. e a lapis di Fucini: «Emilia Peruzzi». Anno, non aut.: 1877?. Inedita.

1. Su Roster cfr. la nota 2 alla lettera XIV.

2. Massimiliano Giarré (Firenze 1830-1888). Nel 1848 partì con i volontari toscani e combattè valorosamente a Curtatone, dove rimase ferito al volto. Tornato a Firenze, dopo aver praticato per alcuni anni 


\title{
CXC
}

\author{
E. PeruZZi a R. Fucini
}

Giovedì sera

La prima edizione viaggiava quand'ebbi la $2^{\text {da }}$ riveduta e corretta ${ }^{1}$.

Grazie mille e di cuore di quella visita a traverso la neve.

La sera di Natale non esco e aspetto lei e la prego di dire ad Uzielli che lo aspetto anche lui ${ }^{2}$. E la Prefazione ${ }^{3}$ !

Sua aff.

\section{CXCI}

\section{E. PeruZZi a R. FuCini}

Caro Fucini, Le nostre due lettere s'incrociarono, La ringrazio molto del gentile desiderio di rivederci, La prego dirmi se Ella potrebbe venire quassù a desinare Giovedì facendo la strada in compagnia di Ubaldino. Se approva me lo scriva e pensi ad iscriversi avanti le 2 di Giovedì fra quelli che vengono all'Antella. Noti che vi sarà soltanto per il ritorno e questo lo dico perché scovandola fra gli iscritti non avessero a aspettarla. Desideriamo molto tutti di rivederla, col brio suo fiorentino rinforzato dal brio napoletano.

la professione di avvocato, nel 1861 ottenne la cattedra di letteratura italiana al liceo fiorentino "Dante"; successivamente, insegnò alla scuola tecnica "Leon Battista Alberti". Fu professore di diritto commerciale, letteratura politica e storia medioevale e moderna presso la scuola di scienze sociali "Cesare Alfieri", della quale divenne anche preside e direttore. Si veda C. Fontanelli, Commemorazione del Cav. Prof. Avvocato Massimiliano Giarré Direttore della Scuola di Scenze Sociali fatta dal professoreCarlo Fontanelli il dì 15 aprile 1888, Firenze, Tipografia dell'Arte della Stampa, 1888.

CXC. Dall'aut. in BRF (FRF, cass. 8, ins. 19). Lettera adespota intestata "EP", 4 ff. su 2 cc. S. d. Inedita.

1. Difficile individuare con certezza a quale opera alluda la Peruzzi. In base al riferimento alla prefazione, si può ipotizzare che si alluda ai Cento sonetti in vernacolo pisano (Firenze, Pellas, 1872).

2. Su Gustavo Uzielli cfr. la nota 2 alla lettera LXXVII.

3. A proposito della prefazione si veda la nota 3 alla lettera LXXVII.

CXCI. Dall'aut. in BRF (FRF, cass. 8, ins. 20). Bigl. vis. adespota intestato "Emilia E Ubaldino Peruzzi". S. d. Inedito. 


\title{
CXCII
}

\author{
E. Peruzzi a R. Fucini
}

\section{Viareggio 18 Piazza d'Azeglio}

Sempre memore.

\section{CXCIII}

CXCII. Dall'aut. in BRF (FRF, cass. 8, ins. 20). Bigl. vis. intestato. S. d. Inedito.

CXCIII. Dall'aut. in BRF (FRF, cass. 8, ins. 20). Busta. Ind.: «Ill.mo Sig. Ing.re Renato Fucini / Empoli / per Vinci). Timbro post.: 23-10-77. 



\section{INDICE DEI NOMI}





\section{INDICE DEI NOMI}

Adami, Giacomo 7n

Albini Bisi, Sofia 113 e n

Ales, Stefano 38n

Alessandri 133 e $n$

Altoviti 72

Altoviti Avila, Vittoria 65 e n, 131 e n, 134 e $\mathrm{n}$

Amari, Michele $74 \mathrm{n}$

Andreucci, Franco 21nn, 23n

Artusi, Luciano 62n

Augello, Massimo M. 66n

Autin, Jean 119n

Avagliano, Lucio 76n

Baccelli, Guido 128 e n

Bagnoli, Paolo 7n,11n, 14n, 21n

Bakounine, Alexandra 108 e $n$

Baldacci, Luigi $7 \mathrm{n}$

Baldi, Fabia 7n

Ballini, Pier Luigi 73n

Barazzuoli, Augusto 65 e n

Barbèra, Prospero 18n, 43n, 57 e n, 58, 59, $145 n$

Barbieri, Lodovico 66n

Bardi, Ubaldo 31n, 127n

Bargellini, Piero 15n, 24n

Bargioni, Gustavo 92 e n, 134 e n

Barret, Elizabeth 31n

Barsanti, Olinto 25n, 114 e nn

Bartolini (famiglia) 15

Bazin, Réne 122 e n, 129 e nn, 124

Bellingeri, Luca 45n

Benedetto da Maiano 89

Beneforti, Giuliano 103n
Benucci, Elisabetta 7n, 8n, 9n, 11n, 13n, 69n, 93n, 110n

Bernhard, 60 e $n$

Bertolozzi, Raffaele 119n

Bertoni Jovine, Dina 963n

Biagi, Guido 8n, 18n, 25 e n, 100n

Bianchini, Marco 66n

Bigazzi, Aldo 52n

Bistino 61 e n, 63, 65, 70

Bodio, Luigi 131 e n, 132

Bolasco 17, 21n, 89, 90

Boldrini, Carlo 65n, 106 e nn

Boldrino, Carlo Emilio 82 e n, 83n, 106 e $n$

Bonamour, Jean 119n

Bonetti, Paolo 76n

Bonghi, Ruggiero 46 e nn, 47, 420n, 145 e nn

Borghese, Lucia $82 n$

Borgia, Salvatore 76n

Branca, Vittore 118n

Brin, Benedetto 100n

Broglio, Emilio 46 e nn, 47, 52n, 61n

Brown, Benjamin 44n

Browning, Robert $31 \mathrm{n}$

Brunelleschi, Irene 21

Bruni, Luigino $77 \mathrm{n}$

Buonazia 85

Cabras, Pier Luigi 120n

Calderini, Marco 98 e n

Cambray, Digny Luigi Guglielmo 61 e n

Cammarota, Gaetano 84 e n, 87 e n, 89 e n, 90 
Campanini, Enrica 120n

Camurri, Renato 21n, 123n

Capanna, Puccio 89 e $n$

Caramelli, Ermengarda 109n, 115n, 118n, 132n, 133n

Carbone, Domenico 94 e $n$

Carducci, Giosue 57n, 113, 114n, 115 e nn, 116, 117, 120n, 123, 129n, $135 \mathrm{n}$

Carli 65n, 72 e n, 73, 105 e n, 106 e n

Carlucci, Paola 11n, 144n

Casati, Alessandro 96n

Casprini, Massimo 31n, 127n

Catarsi, Enzo 63n

Ceccuti, Cosimo $7 \mathrm{n}$

Ciccuto, Marcello 7n

Cini, d on Gaetano 102n

Chiarini, Giuseppe 25n

Chiarugi, Vincenzo 16n, 119n, 120 e n, $127,128 \mathrm{n}, 129$ e $\mathrm{n}$

Chiti, Alfredo 14n, 15n, 17n, 18n, 25n

Ciuffoletti, Zeffiro 14n, 16n, 96n, 98n

Codronchi Argeli, Giovanni 138 e n

Colini, Enrico 42

Colosimo, Giovanni 76n

Contorbia, Franco 7n, 80n

Coppino, Michele 62 e n, 62n, 67, 68

Corsini (famiglia), $44 \mathrm{e} \mathrm{n}$

Coturri, Enrico 120n

Croce, Benedetto 7n, 24n

Cuccoli, Maria Pia 7n, 19n, 42n, 61n, 65n, 81n, 112n, 133n

D'Alcantara, Pedro 33n

D'Ancona, Alessandro 65n, 75 e n

D'Ancona, Sansone 75n, 130 e nn

De Amicis, Edmondo 7n, 8 enn, 9 e nn, 11 e nn, 12 nn, 13 e nn, 48n, 69n, 80 e n, 93n, 110n

De Gori Pannilini, Augusto 58 e n

Del Corona, Ottaviano 37n, 39 e n, 40, 46 e n, 48 e n, 49 e n, 54 e n

Del Lungo, Isidoro 129 e $\mathrm{n}$
De Marchi, Emilio 12, 118 e n

Digerini-Nuti, Andrea 122 e nn, 123, 128 e $\mathrm{n}$

Dillon Wanke, Matilde 7n, 11n

Drumetti 93

Fanfani, Pietro 8n, 47e n, 52n

Farina (famiglia), 90 e $n$

Farina, Emilio 90n

Farina Cini, Neri 90n

Fatichi, Nemesio 21nn, 23n

Federzoni, Luigi 66n

Felisini, Daniela 115n

Ferrante, Lucia 22n, 23n

Fiorentino, Francesco 119n

Fogazzaro, Antonio54n, 123 e n, 125

Fondi, Napoleone 106 e $n$

Fontana Semerano, Sandra 7n

Fontanelli, Carlo 65n, 106e n, 109e n, 146n

Foresi, Raffaelo 8n

Fortunato, Giustino 69n, 70 e n

Fortunato De Lisle, Lucille Mary $7 \mathrm{n}$, $34 n, 54 n$

Franchetti, Augusto 97, 98n

Franchetti, Leopoldo 44n, 82n, 97 e n, $98 \mathrm{n}$

Franzina, Emilio 21n, 123n

Fucini, David 37n, 39n

Fucini, Emma 20, 31n, 36n, 59n, 62n, 85n, 102n, 131n, 132, 135n, 136n, 137,142

Fucini, Enzo 111n

Fucini, Ida 13, 91n, 102n, 111n

Fucini, Nerina $111 \mathrm{n}$

Fucini, Rita 13, 91n, 102n, 111n

Gabardi Brocchi, Gabardo 88 e n, 89, 90

Gabelli 89

Gabbrielli, Silvano 62n

Gajo Mazzoni, Maria Gaia 46n

Gamberucci 89

Gargini, Omero 64 e $n$

Gelli, Agenore 52n, 58 e n 
Genala, Francesco 9, 19n, 21n, 22, 101 e n, 103 e n, 106 e n, 107, 113 e n, 114n, 115 e n, 116 e n, $117,118,121$ e n, 125 e nn, 126 e n, 141 e $n$

Gennarelli Pirolo, Paola $7 \mathrm{n}$

Ghirlandaio, Domenico del 89

Giacalone-Monaco, Tommaso 14n, 23nn, $97 \mathrm{n}$

Giacomelli, Giovan Battista 88 e n, 34 e n, 37 e n, 49 e n, 60 e n, 64 e n, 65

Giacosa, Giuseppe 9n

Giarré, Massimiliano 145 e n, 146n

Gibelli, Vincenzo 119n

Gigli, Lorenzo 8n, 9n, 11n, 80n

Ginori Lisci, Carlo Benedetto 128 e n

Ginori Lisci, Leonardo 44n, 120n, $134 n$

Giorgi, 55 e n

Giorgini, Giovanbattista 12n, 46n, 52n, 61n, 63nn, 65n, 70n, 75n, 107 e n, 109

Giusti, Mariangela $7 \mathrm{n}$

Goldoni, Carlo 37

Gorini Santoli, Angela 19n, 113n, 118n

Gotti, Aurelio 52n, 62 e n, 64n, 65n, 68

Gozzoli, Benozzo 89

Gradilone, Alfredo 107n

Graziano, Luigi 21n

Grifi 72

Guidi, Marco E. L. 66n

Heyse, Paul von 119 e n

Hillebrand, Karl 65n

Homberger, Heinrich 102n, 134n

Hungher, Carolina $74 n$

Iermano, Toni $7 \mathrm{nn}$

Incontri, Ludovico Carlo 64 e n, 95n

Incontri, Maria 108 e n

Jannazzo, Antonio 98n

Jarro (Giulio Piccini) 33n, 111n, 112n

Lama, Ernesto 7n, 24n

Lampertico, Fedele 21n, 123 e n, 125
Leonardo da Vinci 115

Leopoldo II 139n

Linaker, Arturo 88 e n, 113 e n, 136 e n

Lippi, Donatella 120n

Livingstone 245n, 114 e n

Lombardi, Felice 128n

Luzzatti, Luigi 73n

Macry, Paolo 33n

Malandrino, Corrado 77n

Mamiani della Rovere, Terenzio 42 e n, $57 \mathrm{n}$

Manca, Gavino 77n

Manfroni, Angiolina 126

Manfroni, Mario 16 e n, 17n, 72 e n, 83, $84 n, 85,86$ e n, 102n, 126 e n, $134 n$

Mantellini, Giuseppe 77 e nn

Manzoni, Alessandro 11, 12n, 25n, 46 e nn, 61n

Manzoni, Vittoria 63 e n, 70, 75 e n, 145

Marchi, Marco 127n

Marchionatti, Roberto $77 \mathrm{n}$

Mari, Adriano 79 e n, 81

Marraccini, Daniela 15n, 17 e n, 18 e n

Martini, Ferdinando 48n

Mattalia, Daniele 118n

Mauri, Achille 46 e n

Meana 106, 107

Melis, Rossana 7n, 11n, 66n

Mérimée, Prosper 119 e n

Miceli, Luigi Alfonso 108 e n

Michelet, Athenaïs 124 e n

Michelet, Jules 74n, 124 e nn, 125

Milli, Giannina 19 e n

Morelli, Agostino 142

Morelli, Donato 107 e n

Moretti, Mauro 82n

Mori, Maria Teresa 7n, 9n, 23n

Mùndula, Mercede $124 \mathrm{n}$

Musella, Luigi 21n

Nardi, Giovanna 39n

Navarria, Aurelio 12n 
Negri, Ada 19 e nn, 20 e n, 22, 24, 113 e nn, 114 e n, 116 e nn, 117

Negri, Cristoforo $67 \mathrm{n}$

Negri, Eugenio 22, 118, 119n, 121

Nesti, Arnaldo 108n

Niccolai (famiglia) 15

Niccolai, Alberto 8nn, 18n, 31n

Nonni, Ugo 89

Obrenovich, Maria 55 e n

Orsini, Isabella 115 e n

Pacchi 55

Palazzi, Maura 22n, 23n

Palazzolo, Maria Iolanda 7n, 8n

Pancrazi, Pietro 24 e n

Paoli, Baldassarre 58 e n

Paolieri, Ferdinando 24n

Pareto, Vilfredo 9, 14n, 23 e nn, 51n, 76 e n, 77n, 96 e n, 97n, 108 e n

Passerini, Luigi 44n, 134n

Pea, Mauro 19n, 113n

Pellizzari (famiglia) 56n, 74

Pellizzari, Emma 10n, 50n, 56n

Pellizzari, Giorgio 130 e $n$

Peri, Carlo 65n, 106 e n

Perna, Raffaele $101 \mathrm{n}$

Pescetti, Luigi 18n

Pesci, Ugo $114 \mathrm{n}$

Piersanti, Carlo 120n

Pinturicchio 89

Pironti, Carolina 20

Pomata, Gianna 22n, 23n

Porta, Carlo 34n

Prezzolini, Luigi 48 e $n$

Procacci, Giovanni 13, 71n, 97, 98 e n

Proietti, Domenico 8n

Protonotari, Francesco 66n

Rajna, Pio 19n, 20n

Ricasoli, Bettino 48n, 61n, 62n, 64n, 65n,. 139n

Rogari, Ubaldo 7n

Romanelli, Raffaele 33n, 124n
Rosmini, Antonio 46n

Rossi, Giuseppina 7n, 34n, 51n, 131n

Rossi-Cassigoli (famiglia) 15

Roster (famiglia) 37, 132

Roster, Giorgina 131

Roster, Giorgio 10n, 19n, 36 e n, 40 e n, 50n, 56 e n, 59 e n, 61 e n, 62n, 65 e n, 66 e n, 78 e n, 89 e n, 92 e n, 111 e nn, 134 e n, 136, 143 e n, 145 e n

Roster, Maria 131

Russo, Luigi 25n

Rutigliano, Enzo 76n

Sabatier (famiglia) 74 e n, 124, 125n

Sabatier, François $74 n$

Sabatini, Gaetano 115n

Sanesi, Tommaso 94 e n, 95

Sbrocchi, Leonard G. 7n, 13n, 32n, 37n, $71 \mathrm{n}, 83 \mathrm{n}, 84 \mathrm{n}, 107 \mathrm{n}, 121 \mathrm{n}, 135 \mathrm{n}$

Scharten, Teodora 74n, 125n

Schneider, Rodolfo 53

Serao, Matilde 20

Sgroi, Carmelo $7 \mathrm{n}$

Simoni, Renato $15 \mathrm{n}$

Sklovskij, Viktor 119n

Soldani, Simonetta 7n, 79n

Sonnino, Sidney 7n, 9,11 e nn, 44 e n, 58n, 66n, 82n, 96n, 97n, 98n, 128 e n, 144n Spadolini, Giovanni 61n, 65n, 84n, 100n, 139n

Spandre, Silvia $11 \mathrm{n}$

Spaventa, Silvio 46n, 84n

Steinmann, Edmund 20n, 133n

Stromboli, Pietro 84 e n

Tabarrini, Marco 61n, 62n, 64 e nn

Taddei, Maria 24n

Tamburini, Luciano 11n

Tanfucio, Neri 8n, 23, 91n, 134

Tessitore, Fulvio 46n

Tigri, Giuseppe 15n, 75 e n

Tiribilli-Giuliani, Pietro 134n

Titta Rosa, Giovanni 15n

Tommaseo, Niccolò 46 e nn, 57n 
Tommasi Crudeli, Corrado 65 n

Toscanelli (famiglia) 51, 142n

Toscanelli Altoviti Avila, Angiolina 9n, $13 n, 83 n$

Toscanelli, Giuseppe 51n, 65n

Tosto, Eugenio 80n

Traversetti, Bruno 81n

Turgenev, Ivan Sergeevič 119 e n

Ullrich, Hartmut 73n

Uzielli, Gustavo 67 e n, 146 e n

Vagnetti, Italo $127 \mathrm{n}$

Vallardi, Cecilio 119 e n, 120n

Vannucci, Marcello 11n
Vannucci, Vannuccio 33n, 42n, 84n, 92n, 122n, $133 n$

Varanini, Giorgio 8n

Vega, Lopez de 80 e $n$

Villari, Pasquale 13, 69n, 70n, 84n, 99n, 120n

Vimercati, Guido 57 e n, 58n, 79 e n, $96,97 \mathrm{n}$

Zagri, Guido $51 \mathrm{n}$

Zanella, Arnaldo 36 e n, 37n, 60n

Zannetti, Arturo 47 e n

Zanotti-Bianco, Umberto 98n

Zerella, Francesco 42n

Zumbini, Bonaventura 69 e n, 70 
